\title{
Guided by reason, struck by emotion: Integrating motivational and expectancy - value accounts of behavior
}

Citation for published version (APA):

Nelissen, R. M. A. (2005). Guided by reason, struck by emotion: Integrating motivational and expectancy value accounts of behavior. [Doctoral Thesis, Maastricht University]. Maastricht University. https://doi.org/10.26481/dis.20051028rn

Document status and date:

Published: 01/01/2005

DOI:

10.26481/dis.20051028rn

Document Version:

Publisher's PDF, also known as Version of record

Please check the document version of this publication:

- A submitted manuscript is the version of the article upon submission and before peer-review. There can be important differences between the submitted version and the official published version of record.

People interested in the research are advised to contact the author for the final version of the publication, or visit the DOI to the publisher's website.

- The final author version and the galley proof are versions of the publication after peer review.

- The final published version features the final layout of the paper including the volume, issue and page numbers.

Link to publication

\footnotetext{
General rights rights.

- You may freely distribute the URL identifying the publication in the public portal. please follow below link for the End User Agreement:

www.umlib.nl/taverne-license

Take down policy

If you believe that this document breaches copyright please contact us at:

repository@maastrichtuniversity.nl

providing details and we will investigate your claim.
}

Copyright and moral rights for the publications made accessible in the public portal are retained by the authors and/or other copyright owners and it is a condition of accessing publications that users recognise and abide by the legal requirements associated with these

- Users may download and print one copy of any publication from the public portal for the purpose of private study or research.

- You may not further distribute the material or use it for any profit-making activity or commercial gain

If the publication is distributed under the terms of Article 25fa of the Dutch Copyright Act, indicated by the "Taverne" license above, 


\section{Guided by Reason, Struck by Emotion}

\section{Integrating Motivational and Expectancy - Value Accounts of Behavior}

\section{PROEFSCHRIFT}

ter verkrijging van de graad van doctor aan de Universiteit Maāstricht, op gezag van de Rector Magnificus, Prof. mr. G.P.M.F. Mols volgens het besluit van het College van Decanen, in het openbaar te verdedigen op vrijdag 28 oktober 2005 om 12.00 uur door Robert Marie André Nelissen 


\section{Promotor:}

Prof. Dr. N.K. de Vries

Copromotor:

Dr.AJ. Dijker

\section{Beoordelingscommissie:}

Prof. Dr. GJ. Kok (voorzitter)

Prof. Dr.A.H. Fischer (Universiteit van Amsterdam)

Dr. SJ.H.M. van den Putte (Universiteit van Amsterdam)

Prof.Dr.H.de Vries

\section{Colofon}

Printed by Ponsen \& Looijen bv

ISBN: 9076269505

The study presented in this thesis was conducted at the Maastricht Care and Public Health Research Institute (CAPHRI) in the Faculty of Health Sciences of Maastricht University. Caphri is part of the Netherlands School of Primary Care Research (CaRe), acknowledged in 1995 by the Royal Netherlands Academy of Art and Science (KNAW). 


\section{ChAPTER 1. \\ Behavioral Explanation \& Change: \\ The Reasoned Action Perspective}

INTRODUCTION

THE Reasoned ACtION PERSPECTIVE ON BEHAVIOR

An Example: The Theory of Planed Behavior

Some Preliminary intricacies.

The Reasoned Action Perspective on Behavior Change

Expectancy-Value Models in Health Education

How to Change Behavior

\section{ChAPTER 2.}

The 7 Cardinal Sins in Expectancy-Value Research: Comments on the Reasoned-Action Perspective

INTRODUCTION

Model Expansion: InCREASING THE PRedictive POTENTIAL

Additional Antecedents of Attitudes

Differentiating Social Influences

Perceptions of Control

Measuring Intentions

Beyond the Variance Explained

Model Refinement: Specifying the CAUSAL Processes

Between Intentions and Behavior

Determinants of intention-behavior consistency

Automatic processes and habitual action

From Beliefs to Actions

Methodological complications

The direction of causation 
SUMMARY AND CONCLUSIONS

The Effectiveness of Interventions

Cardinal Sins $\quad 36$

Beyond Expectancy-Value Models 37

\section{Chapter 3. \\ On the Origin of Goals \\ And the Role of Emotions in the Control of Behavior}

$\begin{array}{ll}\text { INTRODUCTION } & 39\end{array}$

IDENTIFYING THE GENERAL GOALS OF BEHAVIOR 40

Problems Concerning Previous Conceptions of Fundamental Goals 41

An Evolutionary Approach towards the Identification of General Goals 42

Some comments on the evolutionary approach 44

EMOTIONS AS THE FUNDAMENTAL GOALS OF BEHAVIOR

The Functional Approach to Emotions 45

Emotions as Fundamental Motivational Systems $\quad 46$

Characteristics of Emotional Processes $\quad 47$

Emotion elicitation $\quad 48$

Motivational properties of emotions 49

Emotional influences on cognition $\quad 52$

Identifying Emotions $\quad 54$

Emotions as Fundamental Goals: Summary and Conclusions

STUDV 3.1: RELATIONS BETWEEN EMOTIONS AND VALUES

Introduction $\quad 56$

$\begin{array}{ll}\text { Method } & 58\end{array}$

Results $\quad 59$

Relations between emotion-and value - dispositions 59

Relations between emotions and values in response to behavior

Discussion $\quad 62$

STUDY 3.2: INTERACTIONS BETWEEN EMOTIONS AND CHRONIC GOALS

Introduction $\quad 63$

Emotions and goals 64

Chronic goals and emotions in social dilemmas 64 
Experiment 1 $\quad 65$

Method $\quad 66$

Results $\quad 67$

Discussion 68

$\begin{array}{ll}\text { Experiment } 2 & 70\end{array}$

$\begin{array}{ll}\text { Method } & 70\end{array}$

Results and discussion $\quad 70$

$\begin{array}{ll}\text { General Discussion } & 71\end{array}$

TO WIND UP $\quad 72$

\section{CHAPTER 4.}

Believe what you Want

Integrating Motivational and Expectancy-Value Accounts of Behavior

$\begin{array}{ll}\text { INTRODUCTION } & 75\end{array}$

THE NATURE AND FUnCTION OF OUTCOME EXPECTANCIES $\quad 76$

Factual and Subjective Outcome Expectancies $\quad$ : $\quad 76$

$\begin{array}{ll}\text { The Function of Outcome Expectancies } & 77\end{array}$

The Causal Nature of Outcome Expectancies 78

The Role of Fundamental Goals in the formation of Outcome

Expectancies $\quad 79$

EFFECTS OF GOAL-ACTIVATION ON THE IMPACT OF OUTCOME EXPECTANCIES ON

ACTION $\quad 80$

Emotional Influences on the Accessibility of Outcome Expectancies 81

Emotional Influences on Expectancy and Value Estimates 81

Emotional Influences on OE - Behavior Relations $\quad 82$

Previous Research on Affective Influences on OEs $\quad 82$

STUdY 4.1: EMOTIONAL INFLUENCES ON PERCEIVED OUTCOMES

$\begin{array}{ll}\text { Method } & 84\end{array}$

$\begin{array}{ll}\text { Results } & 85\end{array}$

$\begin{array}{ll}\text { Discussion } & 88\end{array}$

Stud 4.2: GoAl-BASED Matching Of PERSUASIVE MES5AGES 91

Previous Research on Goal-Based Matching $\quad 91$ 
The Present Study

Method

Results

Discussion

GENERAL DISCUSSION

\section{CHAPTER 5.}

"Forbidden Fruit"

\section{Reflections on the Consequences of the Integrated Perspective}

INTRODUCTION

A Summary of the Integrated Perspective

Summary of Research Findings Concerning the Integrated Perspective

CONSEQUENCES FOR EXPECTANCY-VALUE MODELS

Contributions from Incorporating Goals

What about Social Influences and Perceptions of Control?

CONSEQUENCES FOR BEHAVIOR CHANGE BY INFORMATION-BASED HEALTH EDUCATION

Suggestions from the integrated perspective to boost the effectiveness of persuasive messages

FURTHER CONSEQUENCES FOR EFFECTIVE BEHAVIOR CHANGE

CONSEQUENTLY...

Emotions as Goals

The Integrated Perspective 
APPENDIX: GoAl PRIMING BY SEMANTIC DISCREPANCY PRIMES

125

FOOTNOTES

REFERENCES

SUMMARY

155

SAMENVATTING

159

ACKNOWLEDGEMENTS

163

CURRICULUM VITAE 


\section{Chapter 1}

\section{Behavioral Explanation \& Change \\ The Reasoned Action Perspective}

\section{INTRODUCTION}

What do the following individuals, objects, entities, and animals have in common: An angel, a computer, an ant, a Swiss army-knife, Captain Spock (the character from the television series Star Trek), a web or some other kind of network, a thermostat, a blank slate, and a vat with a pressure valve? They are all metaphors used to describe human behavior... Notably, these images all derive from (once) accepted psychological and philosophical conjectures on human nature, the mind, and the generation of behavior. Obviously, in picturing the way our actions come about, scientists paint with quite a colorful and diverse palette. In its broadest conception, this dissertation is concerned with different approaches towards understanding human behavior and with the ensuing implications for inducing behavioral change.

In the course of the following chapters, two perspectives on behavior will be outlined that have constituted the most general frameworks for predicting and understanding action in modem social-psychological theories. In the first two chapters, we will outline the reasoned-action account of behavior, which depicts humans as premeditating agents, deliberating the anticipated consequences of their behavior before deciding upon a particular 
course of action Subsequently, the motivational perspective on behavior will be discussed. According to the motivational perspective, behavior is aimed at the attainment of certain goals that are activated by individual needs or desires and demands and opportunities presented by the environment. We will then develop an integrated view based on both perspectives.

Ultimately, we will investigate if goals can indeed be incomporated into reasoned-action models, as suggested by the integrated perspective. Specifically, we will explore the theoretical contributions, empirical support, and practical consequences (for behavior change) of this integrated perspective.

\section{The Reasoned ACtion Perspective ON BeHAVIOR}

During the second half of the previous century, psychological theories of behavior and motivation were dominated by the reasoned-action perspective (Atkinson, 1980; Feather, 1982). Reasoned-action accounts hold behavior to be the product of people's intentional choice, which is ultimately based on deliberation of the advantages and disadvantages of following a certain course of action.

The popularity of the reasoned-action perspective can be attributed to several factors. First of all, reasoned-action views nicely match people's everyday accounts of their actions, which they ordinarily explain with reference to perceived advantages and disadvantages. Hence, the perspective benefits from an intuitive appeal. Furthermore, it coincides with the (western) ideal, picturing man as a reasonable being who is able to reflect on his/her conduct, which is etemalized in Descartes' phrase: "Cogito ergo sum". Moreover, it nicely fits the scientific niche, abandoned by the behavionist doctrine discarding all explanation with reference to mental states, to which it was invited with renewed enthusiasm after the cognitive revolution. Undeniably, the reasoned-action perspective has also demonstrated to provide a successful account of behavior, both in terms of prediction and understanding, as well as providing new starting points for practical application, as we shall see in the following.

\section{An Bcample: The Theory of Planned Behavior}

The Theory of Planned Behavior (TPB: Ajzen, 1991), which is an adaptation of the Theory of Reasoned Action (Ajzen \& Fishbein, 1980; Fishbein \& Ajzen, 1975) - arguably - constitutes the most popular variant of models framed within the reasoned-action perspective (e.g., Armitage \& Conner, 2001; Eagly \& Chaiken, 1993; Manstead \& van der Pligt, 1998).

The model was proposed as a parsimonious framework to understand and predict volitional behavior (Ajzen \& Fishbein, 1980). The TPB (see Figure 1.1) suggests that the proximal determinant of behavior is one's intention to engage in the act. Intention thus serves as an index of a person's motivation, in the sense of his or her conscious plan to engage in an act. So, my buying extra-soft toilet paper at the supermarket tonight, primarily depends 
on my intention to do so. Intentions are determined in concert by people's attitudes, subjective noms, and percerved behavioral control.

An attitude is the overall positive or negative evaluation of performing the act fi.e, whether I think buying extra-soft toilet paper is a good or bad thing to do). According to the TPB, attitude formation is the result of a deliberative process in which individually salient outcome expectancies are judged in tems of expectancy (meaning the perceived likelihood that performing the behavior will cause this outcome) and value (the positive or negative evaluation of this outcome).

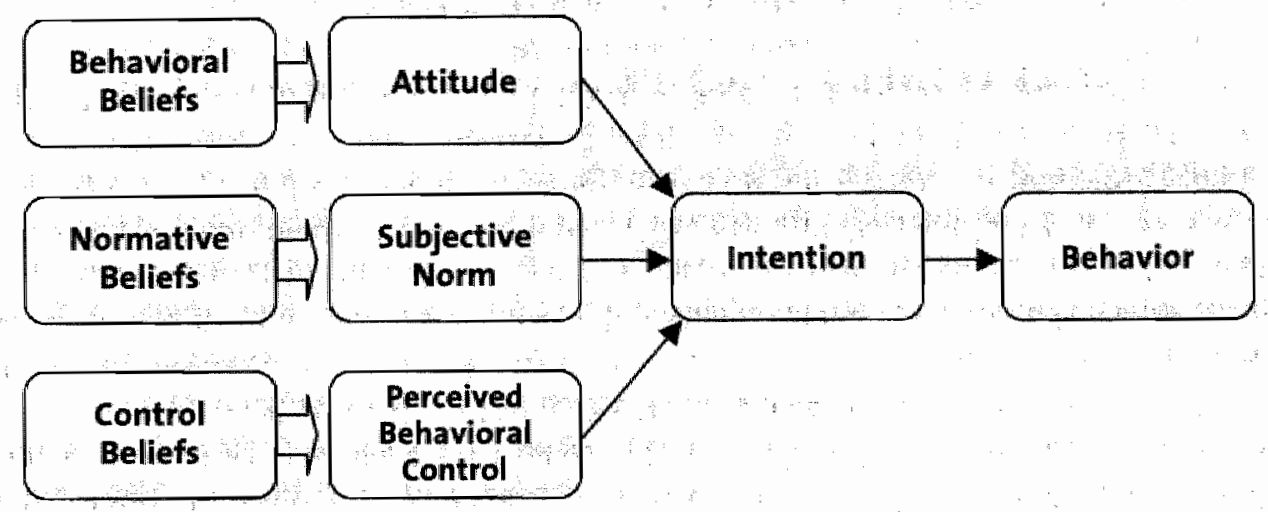

Figure 1.1. Schematic Representation of the Theory of Planned Behavior.

For instance, I may believe that when I buy extra-soft toilet paper, wiping my bottom the next time I'm on the toilet, will feel very comfortable, which I deem very positive. On the other hand, I will also have to spend more money on extra-soft toilet paper, which I find negative. According to the model, attitudes are determined by the summed product of expectancies and values of beliefs concerning the possible consequences of action that are individually salient.

Similarly, subjective norms are also a product of beliefs, but of a different nature. The subjective norm concerns individuals' perceptions of what other, personally important people would like them to do. The subjective norm is a multiplicative composite of one's normative beliefs (i.e, concerning the opinion of important referents) and the motivation to comply with them, just like the attitude is of behavioral outcome expectancies and their evaluation. Conceming my decision to buy extra-soft toilet paper, I may be compelled to do so because my girlfriend urges me to buy extra-soft toilet paper. On the other hand, were she an environmentalist, she may rather have wanted me to get the unbleached, rough paper, which had put me in a pickle.

Finally, perceived behavioral control refers to the extent a person feels he or she has control over performing the behavior, and is usually assessed by perceptions of how easy or difficult it is to perform the behavior. For instance, the local supermarket, being a prize- 
fighter, may only sell quantum-packs of the crappy kind of toilet paper. Hence, to bury the extra-soft paper I would have to go to another supermarket much farther away from where I live, which due to crowded traffic, might be closed by the time I get there. Perceived behavioral control was introduced into the model to account for actions that are not entirely under the volitional control of the actor, in spite of sufficiently high intentions (Ajzen, 1991). According to the TPB, perceived behavioral control, apart from influencing people"s intentions, also exerts a direct impact on behavior. The rationale behind this direct link is that, holding intentions constant, people are more likely to engage in actions they believe are more executable than in actions they consider difficult to perform.

The TPB, along with its predecessor, the theory of reasoned action, has been used to predict an immense variety of different actions (see: Ajzen, 1991; Amitage \& Conner, 2001; Godin \& Kol, 1996, for reviews), ranging from the private (eg., breast and testicular selfexamination) to the interpersonal (e.g., leaving abusive relations), from work-related activities (e.g, studying and job search) to the things we do to relax (e.g, leisure activities, alcohol consumption), and from altruistic (eg, blood-donation, pro-environmental behavior) to selfish (e.g., whistle-blowing) aspects of our conduct It is probably precisely because of its parsimonious nature, its wide applicability, and its relative accuracy of prediction (more of which in Chapter 2) that the TPB still enjoys unceasing popularity. A recent count on the database of PsychInfo, yielding 379 hits for publications from the year 2000 onward on the search string "TPB", attests thereof. (Note that this count actually presents a very conservative estimate of the populanity of reasoned action models in general, of which the TPB is but a single exponent)

\section{Some Preliminary Intricacies}

Two conspicuous aspects of the model already deserve our attention here, for they will feature prominently throughout the present discussion. The first concems the measurement of the variables in the model. A classic notion in social psychology states that specific actions cannot be predicted from global motives (eg., Eagly \& Chaiken, 1993). For instance, my attitude towards soccer will not be a reliable predictor of my attending tonight's soccer match at the stadium, neither will my attitude towards extra-soft toilet paper predict my buying it at the supermarket tonight. This weak correlation between general attitudes and behavior almost caused a crisis in the field of social-psychology (e.g. Wicker, 1969), to which attitudes were deemed "the most distinctive and indispensable construct" (Allport, 1935, p. 798). It was the brilliance of Fishbein and Ajzen (1975) to note that for attitudes to be reliably predictive of behavior, both had to be specified at a similar level of abstraction. Formalized as the "comespondence principle", they argued that all measures used to predict a specific behavior should match in terms of action (buying), target (extra-soft toilet paper), context (at the supermarket), and time (tonight). Consistent with their view, numerous studies have shown that when measures correspond, behavior can be reliably predicted from intentions, attitudes and other variables (Ajzen \& Fishbein, 1980). 
A second aspect concems the fact that many variables that have traditionally been of interest to social-psychologists, like gender, socio-economic status, and personality traits, are omitted from the model. Although the importance of such influences is not denied, according to the TPB, they should be considered "distal" variables that only affect behavior indirectly, that is, through relations with one of more of the variables specified in the model (Ajzen \& Fishbein, 1980). Distal variables are to be differentiated form additional variables that influence intentions and behavior directly, that is, independent from the variables already specified by the model. The latter can be incorporated into the model if proven to increase its predictive potential

Both the issues of correspondence in measurement and the omission of external variables will receive greater attention in the next chapter.

From the description of the TPB it may be clear why models formalizing a reasonedaction perspective are commonly refenred to as "Expectancy-Value" (EV) models of behavior (Atkinson, 1980; Feather, 1982): Because they assume that the process by which behavior, intentions, and attitudes are generated can be captured by a mathematical function of people's evaluations and expectancies concerning the perceived outcomes of performing an act Similarly, the reasoned-action perspective has also been addressed as the "consequentionalist perspective", as its main focus of explanation concems the perceived consequences of behavior (cf. Ioewenstein, Weber, Hsee, \& Welch, 2001). In healthpsychology, and particularly in the field of health-education, EV models are known as "socialcognition" models of behavior (Armitage \& Conner, 2000). We will turn to the place of EV models - the term that will be maintained throughout the rest of this discussion - in healtheducation practice, in the subsequent paragraph.

\section{The Reasoned Action Perspective on Behavior Change}

In the present paragraph, we will outline the implications of adopting a reasoned-action perspective on behavior for attempts to induce behavior change. The topic will be related to health-education practice, although similar views have perspired, for instance, in marketing and consumer research (Solomon, 2002).

\section{Expectancy-Value Models in Health Education}

Health education is a way of health promotion. The latter concems the educational, political, regulatory, and organizational means to support the health of individuals, groups, or communities. Traditionally, health education is defined as a planned activity that stimulates leaming by means of communication to promote healthy behavior (e.g. Green \& Kreuter, 1991). Nowadays, health-education activities encompass a much wider range of methods and strategies for effectuating behavior-change than mere communication (Brug, Schaalma, Kok, Meertens \& van der Molen, 2001). Yet, we will concentrate on the transfer of 
information as this approach immediately follows from adopting a reasoned-action perspective and still constitutes a key element of health-education practices. Below, we will elaborate on the various aspects of this definition for health education:

First, health education is a planned activity that proceeds through several stages from the recognition of a health-problem, the identification of environmental and behavioral determinants of the problem, and the analysis of the determinants of the behavioral component of the problem, which are to be targeted in interventions aimed at inducing behavioral change. Subsequently, after development and implementation of the intervention program, it is evaluated with reference to the initial program goals (e.g, Green \& Kreuter, 1991; Brug, et al, 2001). Although this sequence may seem obvious, it is nevertheless often disregarded. The adoption of a systematic approach, however, constitutes one of the major determinants of the effectiveness of an intervention (Kok, van den Bome, \& Dolan Mullen, 1997).

Social-psychological theories feature prominently throughout all stages of planned health education and their application is essential to a planned approach of health education (e.g. Kok, Schaalma, De Vries, Parcel, \& Paulussen, 1996). In this sense, we often speak of "theory-based health education", as the assumptions guiding the actions undertaken along the various phases in the health-education process are derived from theoretical specifications conceming their consequences. Our focus concems the use of theories in the analysis of behavioral deteminants. The popularity of the reasoned-action perspective therein is reflected in the wide variety of social-cognition models identified in the literature (see: Armitage \& Conner, 2000; Kok et al, 1996; Redding, Rossi, Rossi, Velicer, \& Prochaska, 2000, for overviews). Each model concentrates on somewhat different considerations for engagement in (health-related) action and includes somewhat different variables. Nevertheless, they commonly share the same underlying EV structure (Weinstein, 1993). According to the Health Belief Model (Janz \& Becker, 1984), for instance, the likelihood that someone will engage in action to prevent illness depends (a.o) on (the product of) someone's perceptions of his or her vulnerability to the condition and the perceived severity of the condition. Similarly, according to Protection Motivation Theory (Rogers, 1983), a person's motivation to undertake protective action against negative health consequences is determined by threat appraisals, which are also the product of perceived vulnerability and severity. Expectancies conceming the outcome of a particular action and the value attached to this outcome by the individual are also a part of Bandura's (eg., 1986) Social Cognitive Theory and Subjective Expected Utility Theory (Edwards, 1954) as they are of the Theonies of Reasoned Action and Planned Behavior (Ajzen, 1991; Ajzen \& Fishbein, 1980).

\section{How to Change Behavior}

As stated in the definition, health education aims to promote healthy behavior by means of learning through communication, which implies a voluntary process on behalf of the individual as opposed to other health-promotion measures, such as legislature and pricing (Kok, et al, 1996; Bnug et al, 2000). From a reasoned-action perspective on behavior, the 
primary means to stimulate this leaming process is through the transmission of infarmation. Specifically, the EV models cormmonly applied in health-education research, imply that behavior change can be achieved through changing people's considerations that underlie their motivation to engage in a certain activity or not According to the TPB, there are three strategies for achieving this aim changing existent salient beliefs (i.e, in terms of expectancy or value), making existing, non-salient beliefs salient, and/or inducing new salient beliefs (e.g. Ajzen \& Fishbein, 1980; Eagly \& Chaiken, 1993; Hardeman, Johnston, Johnston, Bonetti, Wareham, \& Kinmonth, 2002; Sutton, 2002b).

For instance, condom use can be enhanced by increasing the endorsement (i.e., expectancy) that condoms use will reduce the risk of becoming infected with HIV. (Or conversely, as recently attempted by the roman-catholic church, condom use might be reduced by advocating that because of microscopically small holes, condoms are ineffective in stopping the HIV virus.) Also, the (positive) evaluation of this property of condoms can be increased by stressing the hamful consequences of getting AIDS. Furthermore, additional expectancies that will promote the use of condoms can be stressed, for instance, the use of condoms as a means of birth control or one's obligation to use condoms to protect one's partner from contracting a disease.

The communication of information with the objective of changing people's considerations conceming their health-related activities is also known as "persuasive communication": Persuasion can be defined as: "a successful, intentional effort at influencing another's mental state through communication in a circurnstance in which the persuadee has some measure of freedom" (e.g., OKeefe, 1990, p.17). Note that this definition by and large shares the same characteristics as the (traditional) conception of health education in general Both specify a planned / intentional (as opposed to a chance) process, based on communication of information, aimed at inducing enduring change (i.e., through leaming), that is voluntarily adopted by the receiver. This definitional similarity illustrates the central position of persuasive communication in health-education practice. Originally, research on persuasion set out to investigate the various aspects of the source and content of a message as well as characteristics of the receiver that determined the effectiveness of a message (e.g, Hovland, Janis \& Kelley, 1953). From this have spawned many theonies on successful communication, like McGuire's (1969) "persuasion-communication model". Many variables, apart from message content, have been found to influence the effectiveness of persuasive communication at changing people's beliefs (see: O'Keefe, 1990, for an overview).

Nevertheless, this approach to behavior change ultimately stands or falls by the validity of the psychological processes as described in EV models, hence, by the presumed causal impact of anticipated outcomes on people's motivation to engage in action. We shall see that, though they are not essentially incorrect, several aspects of causation, however, do remain implicit in EV models, which is he primary reason for proposing an integrated perspective. 


\section{SUMMARY AND OVERVIEW}

In this introductory chapter we outlined the reasoned-action perspective on behavior as formalized in EV models, such as the TPB. According to this view, behavior is considered the result of an intentional choice based on people's considerations conceming the perceived consequences of engaging in action The practical implication directly ensuing from this perspective, is that behavioral change; for instance in a health education context, can be effectuated by the communication of information to influence the considerations (i.e., expectancies) underlying people's actions. The following discussion, as will be presented throughout the chapters in this book, constitutes an investigation into the psychological validity of the reasoned action perspective, and incidentally of the tenability of its practical implication. We will argue that the incorporation of goals may substantiate the causal processes as outlined in EV models, but also highlights the reactive nature of behavior, resulting from the situational activation of motivational processes, which is not accounted for by the reasoned-action perspective.

In order to determine its psychological validity, Chapter 2 presents an overview of the various comments on the reasoned-action perspective that have accumulated during the past decades. From this, several ways for model improvement can be identified. We will focus on certain problems surrounding the presumed causal influence of outcome expectancies on behavior. Specifically, we will see that the causal nature of outcome expectancies does not necessarily follow from their conceptualization in current EV models. It will be argued that in order to account for the motivational nature of outcome expectancies, they should be related to the operation of the fundamental goals underlying human behavior.

In order to establish associations between outcome expectancies and goals, we present an outline of the motivational perspective on behavior in Chapter 3. According to the motivational perspective, behavior is aimed at the attainment of certain goals, which ultimately derive from evolutionary requirements imposed by natural selection. We will argue that the spectrum of human emotions may serve as an index of these goals and present two empinical studies investigating the proposed relation between emotions and goals. An extensive discussion will be provided of the processes by which emotions are activated from the detection of cues in the environment, how they motivate our behavior, and orchestrate the various cognitive processes involved in generating an adaptive behavioral response.

In Chapter 4, we integrate the reasoned-action and motivational perspectives on behavior by linking outcome expectancies to goals. Basically, we will argue that outcome expectancies specify behavioral consequences with reference to the attainment or frustration of the general goals as outlined in Chapter 3. Based on this proposition, several expectations can be derived concerning the impact of activating general goals on the evaluation of outcome expectancies and their relation with behavior. Furthermore, the goal- 
based nature of outcome expectancies would incur that the matching of persuasive messages to individual or situational variation in the level of goal-activation should enharice its effectiveness in inducing attitude or behavior change. Twoempirical studies are presented in which these expectations were tested.

Finally, in Chapter 5, we will consider how the integrated perspective, in which the impact of outcome expectancies is related to the activation of goals, may impose boundaries on the potential to change behavior by means of communication, hence may reduce the tenability of the reasoned-action perspective on health education. We will argue that acknowledgement of the integrated perspective urges an increased understanding of emotion-control processes. 


\section{Chapter 2}

The $>$ Cardinal Sins in Expectancy $-V$ alue Research Comments on the Reasoned Action Perspective

\section{INTRODUCTION}

In spite - or perhaps - because of the aforementioned populanity (see Chapter 1), EV models were frequently subjected to critical discourse during the past decades. This chapter presents an inventory of these criticisms, comments, evaluations, and assessments, along with an overview of the most conspicuous modifications and extensions to the basic EV model that have ensued thereof.

The discussion is frequently based on reflections concerning the TPB as this is, as already stated, the most widely applied variant of current EV models (Eagly \& Chaiken, 1993; Manstead \& van der Pligt, 1998), especially in the field of health education (Hardeman, Johnston, Johnston, Bonetti, Wareham, \& Kinmonth, 2002), and arguably, constitutes "the dominant account of the relationship between cognitions and behavior in social psychology" (Cooke \& Sheeran, 2004, p. 159). Representing a blueprint for the reasoned-action perspective - taking the TPB as a starting-point, therefore, ensures both a comprehensive and a representative record of accumulated comments. Yet, given the integral nature of this survey, we will not dwell on model-specific comments but only address criticism that concems the reasoned-action perspective in general. 
Although the status of EV models is already abundantly evaluated previously, most contributions to this debate have mainly focused on specific qualities of a model, like predictive validity, methodological considerations ( $\mathrm{eg}$, concerning the measurement of particular variables), or the relation between certain variables. Consequently, proposals for modification and extension have also been largely domain specific, providing solutions only with respect to a specific point of concem, often disregarding other contributions. The latest comprehensive assessment of accumulated comments (Conner \& Armitage, 1998) dlates back for the better part of a decade.

The present discussion complements previous evaluations by providing a complete and structured survey of both earlier and more recent comments to EV models. Furthemore, it addresses the various extensions proposed and outlines their commonalities and interrelations. Finally, this chapter is set apart from previous overviews as we will not discuss every contribution in great detail, but primarily elaborate on the reactions (both empirical and theoretical) engendered by the various contributions. As such, this chapter mainly presents meta-commentaries ( $\mathrm{i} \mathrm{e}_{\mathrm{y}}$ comments on comments) to $\mathrm{EV}$ models. Indeed, our main goal is not - like that of most previous reviews - to decide on the best direction for future research, but rather to provide a well-informed index of things-that-should-no-longer-beneglected in order to prevent stagnation. These will be summarized as "the 7 cardinal sins in expectancy-value research". This aim follows from our conviction that it is impossible to judge on the potential merits of any kind of contribution not yet fully explored, whereas existing data may well indicate dead-end streets.

This chapter is organized as follows: The next paragraph lists various constructs that have been proposed as extensions to the core EV model in order to boost its predictive value. We will then outline the comments raised against this way of model improvement, before tuming to an evaluation of the causal status of the psychological processes described in EV models in paragraph three. Specifically, we will focus on the two basic assumptions of the reasoned-action perspective; which - to recapitulate - constitute the assumption that (1) behavior is ultimately the result of an intention, (2) based on a deliberative process of weighing the pro's and con's of acting. Research evidence will be discussed that attenuates the former assumption. More importantly, however, it will be argued that the causal influence of cognitions is ambiguously specified in current EV models, which renders their potential for theory-based interventions problematic The final paragraph provides a summary of the present discussion, outlines the major pitfalls in EV research (summarized as the seven cardinal sins), and introduces our argumentation conceming the causal nature of cognitions that will be elaborated in subsequent chapters. 


\section{MOdel EXPANSION: INCREASING THE PREDICTIVE POTENTIAL}

Generally, one feature is singled out above all others as a touchstone of success or failure for any model: the percentage of explained variance as an index of its predictive validity. Metaanalyses on the efficacy of the TPB (Armitage \& Conner, 2001; Godin \& Kok, 1996; Van den Putte, 1991) and other EV models (Sheeran, 2002), indicate that model variables usually account for $30-40 \%$ of variance in intentions, which in tum account for $20-30 \%$ of variance in behavior.

It should be noted, however, that the latter percentages incline towards the lower end of that range in case behavioral measures are assessed objectively instead of by self-reports (Armitage \& Conner, 2001; Sheeran, 2002): Also, these numbers are apt to be inflated due to common utilization of the principle of correspondence as prescribed by Ajzen and Fishbein (see Chapter 1). As a result, interrelations between variables tend to be strong, not because the model so accurately captures the psychological processes under investigation, but rather because of semantic similarities in the phrasing of the questions used to measure the various components of the model (e.g., Eagly \& Chaiken, 1993; Sutton, 1998; Swanbom, 1996; Weinstein, 1993). For instance, one could question whether items like, "My going to the U2 concert with my friends next Friday at the music hall is: good - bad" (measuring attitude) and, "I intend to go to the $\mathrm{U} 2$ concert with my friends next Friday at the music hall is: likely unlikely" (measuring intention), are experientially differentiated in the mind of an average respondent.

Keeping these considerations in mind, the question remains whether explaining this much variance should incur disappointment or contentment. This of course, depends on the standard of comparison one applies to evaluate the amount of variance explained (Sutton, 1998). According to Cohen's operational definitions of effect sizes typically found in the behavioral sciences, EV models have a moderate effect size where it comes to predicting intentions, but only a small effect size for predicting behavior. Obviously, the numbers reported above deviate considerably from the ideal $100 \%$ maximum.

Sutton (1998), however, addresses several reasons to elucidate why the $100 \%$ norm should be considered unrealistic. For instance, intentions may change before behavior is executed and may therefore prove a poor predictor with increasing lag of measurement between both variables. Secondly, violations of the principle of correspondence may diminish observed relations but, as argued before, may also contribute to artificial enhancement thereof. Several other causes, related to flaws in design and measurement (eg., unequal response categories, extreme population samples) may also account for some deviation from the $100 \%$ criterion. According to common consensus; however, the suboptimal predictive validity primarily illustrates that present EV models are far from complete and additional variables are required as determinants to improve behavioral predictions (e.g., Abraham, Sheeran, \& Johnston, 1998; Conner \& Armitage, 1998; Eagly \& Chaiken, 1993; Manstead \& Parker, 1995; Sutton, 1998). 
Indeed, numerous propositions have been made to expand the basic EV model in order to increase its predictive potential Contributions roughly fall into either of four dasses of constructs, related to distinct types of determinants of (health-) behavior (ff. Abraham, et al., 1998). These contributions constitute (1) additional antecedents of attitudes; (2) different normative influences, (3) variations in control beliefs, and (4) indices of intention The subsequent sections present an overview of these contributions.

\section{Additional Antecedents of Attitudes}

EV models are often criticized for their one-sided emphasis on cognitive (i.e., knowledgebased) outcomes and the apparent neglect of affective antecedents (i.e, based on feelings) of attitudes and behavior (e.g. Eagly \& Chaiken, 1993; van der Pligt, Zeelenberg, van Dijk, de Vries, \& Richard, 1998). Mirroring the general attitude towards affect in the field as a whole, original EV models did not attribute an independent status to affective influences in attitude formation or as determinants of behavior (Loewenstein, Weber, Hsee, \& Welch, 2001). For instance, in the original formulation of the TPB, Ajzen (1991) argued that affective responses depend primarily on cognitive inferences and only influence the direction and intensity of outcome evaluations.

Nowadays, a large body of evidence has accumulated that attests for an independent role of affective influences in the formation of attitudes and the generation of behavior (for overviews; see: Haddock \& Zanna, 1999; Manstead \& Parker, 1995; van der Pligt \& de Vries, 1998b; van der Pligt et al, 1998). For instance, Manstead and Parker (1995) showed that utilitarian (cost-benefit) considerations concerning the outcome of committing speeding violations ( $e . g$. "Speeding will make me be in time for my appointment"), correlated only modestly with affective evaluations, defined as positive or negative feelings about performing the act (e.g. "Speeding makes me feel excited"). Furthermore, the relative contribution of both types of evaluations to overall attitudes, appears dependent upon the type of behavior under investigation (Haddock \& Zanna, 1998; Pooley \& O'Connor, 2000). For example, affective evaluations were better predictors of intentions towards smoking, whereas cognitive evaluations were better predictors of studying, indicating behavioral differences in the relative strength of affective and cognitive antecedents (Trafimow, Sheeran, Lombardo, Finlay, Brown, \& Armitage, 2004). There also appear to be individual differences in the extent to which affective and cognitive evaluations affect people"s attitudes (Haddock \& Zanna, 1999; Trafimow et al, 2004).

Other research supporting the distinction shows that affective and cognitive components of attitudes are caused by different expeniences. Direct experience with a particular attitude object (puzzles), generated affective evaluations, whereas indirect experience, through communication, resulted in cognitive evaluations (Millar \& Millar, 1996). Moreover, the latter appeared more predictive of subsequent instrumental behavior (playing with the puzzle as a means of training one's analytical skills). Affect-based attitudes on the other hand, proved better predictors of consummatory behavior (playing with the puzzles just for fun). Finally, attitudes based on affective evaluations were found to be more 
accessible in memary than cognition-based attitudes (Verplanken, Hofstee, \& Janssen, 1998). Although several explanations may account for this latter finding, it undersoores Zajonc's (1980) claims on the primacy of affective evaluations and corroborates evidence indicating that attitudes based on direct experience, hence with a primary affective component; are better predictors of behavior (Fazio \& Zanna, 1981):

Apart from affective evaluations associated with engagement in behavior, several studies show that behavioral differences can also result from individual variation in affect anticipation (van der Pligt, et al, 1998). Anticipated affect refers to expectations of affective consequences of performing a certain act In a series of studies, Richard, van der Pligt, and de Vries (1996) demonstrated that behaviors like, condom use, eating junk food, drinking alcohol and using soft drugs, elicited negative anticipated post-behavioral responses, and that these responses were more negative than the global attitudes towards these behaviors as well as the affective evaluations thereof. Furthermore, they found that whereas the global evaluations (attitudes) did not significantly differ from the affective evaluations, anticipated affective reactions differed substantially from both, supporting the discriminant validity of anticipated affect measures. Other experiments (Richard, de Vries, \& van der Pligt 1998), showed that increasing the salience of anticipatory emotional reactions to having unprotected sex, increases the reported frequency of precautionary sexual behavior. Altogether these results provide impressive support for the inclusion of measures of anticipated affect in EV models, as this would greatly enhance their practical utility in designing behavior change interventions (Conner \& Armitage, 1998).

Nevertheless, debate still remains concerning the actual place of the anticipated affect construct in the overall EV model. A two-factor model, in which attitude and anticipated affect were included as separate factors, appeared to provide a better fit than a single-factor model in which both were combined (Richard, et al, 1996). Nevertheless, this may have been the consequence of the measures applied closely resembling attitudinal scales (e.g." "My eating junk food would make me feel.. pleasant-unpleasant"). Applying more belief-like measures (e.g, "My eating junk food would make me feel unpleasant is... good-bad; likelyunlikely") may have yielded altogether different results (Conner \& Amitage; 1998). Future studies must still resolve some of these measurement issues surrounding the assessment of affective components. Also, researchers have only just begun to explore the potential contributions of more differentiated measures of affect, assessing specific (anticipated) emotional responses to behavior (e.g. Giner-Sorolla, 2001; Manstead \& Parker, 1995; van der Pligt et al, 1998; Zeelenberg, van Dijk, Manstead, \& van der Pligt, 2000)

Few researchers nowadays still challenge the independent and profound impact of affective processes. Still, some argue that the way in which affective measures are currently incorporated in EV models does not quite capture the actual nature of affective and emotional processes on behavior (e.g., Loewenstein et al, 2001). Briefly stated, they argue that present operationalizations represent but a form of cogniticized or "expectation-based" affect that does not correspond with the reactive nature of emotional influences on behavior in a given situation. (More of which in Chapter 3.) Ultimately it may even be altogether 
impossible to adequately capture emotional influences in the anticipatory manner that is inherent to EV modeling of behavior... But before getting too far ahead of things we'll better leave these matters to subsequent chapters and retum to our discussion of EV models. Other contributions suggest that, apart from EV estimation of outcome expectancies, additional factors influence the decisions to engage in behavior.

\section{Differentiating Social Influences}

Research has identified a scope of social influences affecting people's behavior (see: Abraham et al, 2001; Conner \& Armitage, 1998, for overviews). Social influences can be defined as the processes whereby people directly or indirectly influence the thoughts, feelings, and actions of others (Bandura, 1986; De Vnies, Backbier, Kok, \& Dijkstra, 1995). A primary distinction can be made between situational-and cognition-mediated social influences (cf. De Vries, et al, 1995). As many health-related behaviors (e.g., drinking and smoking) take place within social settings (e.g., in bars or at parties), the former are apt to play an important role as determinants of behavior. Situational social influences constitute, for example, social pressure (e.g., remarks about drinking rate) and conformity influences (e.g, smoking to be part of a peer group). Within the cognitively mediated influences, a distinction can be made between injunctive social norms, representing beliefs about others' (dis-) approval of ones' actions (like the social norm construct from the TPB) on the one hand, and descriptive social norms on the other (cf. Cialdini, Kallgren \& Reno, 1991, as cited in: Abraham at al, 2001). The latter constitute, for example, modeling influences (Bandura, 1986) that stimulate imitation of behavior in similar situations.

Furthermore, normative beliefs may not only derive from other people but also from personal standards. Ajzen (1991) himself suggested the TPB could improve from incorporating "moral norms", defined as an individual's perception of the moral (in-) correctness of performing a behavior. Moral norms should have an important influence on the performance of actions with ethical dimensions (like donating to charity causes), the enactment of which reflects important societal values (e.g, helping people in need). Closely related to moral norms, "personal noms", reflecting the values of an individual rather than of society as a whole, have also been demonstrated a distinct normative influence (Cialdini, Kallgren \& Reno, 1991, in: Abraham at al, 2001). For example, even without expeniencing any moral restrictions, a person - having a set of personal principles associated with the behavior - may feel an urge to refrain from eating fast food as he considers himself a healthy person. The personal norm concept, in turn, shares a large resemblance with "self-identity", which is described as the salient part of an actor's self which relates to a particular behavior (Sparks \& Guthrie, 1998). People may engage in a variety of actions (e.g, only eating free-range eggs and organic products, separating their garbage, not driving a car) because they see themselves as thereby fulfilling a particular role (being someone who is concerned with environmental issues). As such, behavior can also be motivated by people's need to maintain their selfconcept. 
Although it seems clear that various social and normative pressures operate to guide our actions in different contexts, several questions still remain unanswered. First of all, it is difficult to disentangle the relative importance of situational and oognitively mediated influences, as this requires the application of different research methods (Oostveen, Knibbe, \& De Vries, 1996). Specifically, normative influences are usually measured in survey research, whereas observational techniques are required to determine the impact of direct social influences occurring in the actual behavioral setting. Also, further research is required to test the extent to which the various nomative concepts can actually be differentiated from one another and to detemine their impact on a wider scope of behaviors (Conner \& Armitage, 1998).

\section{Perceptions of Control}

Thirdly, it has often been remarked that EV models encounter difficulties in accounting for non-volitional factors (e.g, resources, skills, opportunities) that affect the execution of behavior. The Theory of Reasoned Action (Ajzen \& Fishbein, 1980) tried to circumvent this problem by restricting the scope of prediction to the perfomance of specific actions (e.g., going to the gym twice a week, studying every evening), rather than the attainment of outcomes or goals (eg, losing weight, passing for an exam). Although this restriction may be reasonable, as a consequence, the model to al large extent forfeited its potential to generate understanding of complex behaviors, involving sequences of actions and the attainment of general goals, which is often of most interest to social psychologists (ff. Eagly \& Chaiken, 1993).

Moreover, it became apparent that even quite simple acts have their own potential problems of execution and that in that sense; every act needs to be regarded as a goal in itself (Ajzen, 1991; 2002a). For instance, even going to the gym can be considered of constituting several intermediate acts (e.g., joining a gym, packing the necessary sports gear, traveling to the gym's location), each of which may be hindered by uncontrollable factors or events (e.g., finding no nearby gym, not having the money to buy the appropriate gear, missing opening ours because of a traffic jam). Ajzen (1991) introduced the concept of perceived behavioral control (PBC), in order to take into account such control factors like the necessary resources, skills, and opportunities.

PBC is usually assessed by asking how easy or difficult it would be to perform a particular action Supposedly, PBC is one of the determinants of intentions and also exerts a direct impact on behavior. Both relations, however, have been questioned (e.g. Eagly \& Chaiken, 1993; Sutton, 2002a). The direct influence on behavior is explained by arguing that the greater the control a person has over the behavior, the greater the chances that intentions will actually result in the successful execution of behavior. According to this account, the control construct taps the amount of actual control, and holds only to the extend that PBC is a reliable proxy thereof, hence, encompasses a reliable estimate of factors such as the availability of opportunities and resources that are prerequisites for engaging in the behavior, which is debatable. Beyond that, a certain amount of direct control could be considered a 
prerequisite for the rnodel to be applicable in the first place and as such, the direct influence of PBC on behavior is theoretically uninteresting (Greve, 2001; Sutton, 2002a). The rationale behind the impact of $\mathrm{PBC}$ on intentions is that the more confident people are they can successfully execute a particular action, the more they will be motivated to do so. As such, the control constructs assesses perceptions of capacities and skills necessary to perform a given behavior. Nevertheless, this suggests that people would engage in action merely because they feel confident they are able to. Yet obviously, whereas virtually everyone may feel capable to jump of a 12 -story building, not that many people actually do so. In other words, a more accurate representation of the possible influence of PBC may lie in its functioning as a moderator of intention-behavior relations, enhancing enactment only to the extent that people hold positive intentions towards a particular behavior (cf. Eagly \& Chaiken, 1993).

The dual influence thus implies that PBC actually reflects two control processes that bear little or no relation to each other (Sutton, 2002a). One closely resembling Bandura's (1986, 2001) concept of "self-efficacy", which refers to people's beliefs about their capabilities to exercise control over their own level of functioning and over events that affect their lives. The other refers to perceptions of the extent to which behavior is actually under volatile control, as opposed to external influences. This conception, also referred to as "perceived control", closely matches Rotter's (1966) "locus of control" concept, which specifies the extent to which people perceive their behavior as caused by internal (intentional or volatile) or external (environmental barriers or facilitators) factors. A growing body of evidence, across a variety of behaviors, supports this distinction (Manstead \& van Eekelen, 1998; Trafimow, Sheeran, Conner, \& Finlay, 2002. But see: Ajzen(2002), for a different interpretation).

Nevertheless, although both control factors can be separable, they may still influence each other (Conner \& Armitage, 1998). Situations perceived as uncontrollable due to extemal factors may reduce self efficacy and vice versa. It is therefore argued that in order to gain full understanding of the difference between self-efficacy and perceived control, it is necessary to first examine the underlying control beliefs instead of relying on direct measures only (Armitage \& Conner, 2001; Conner \& Armitage, 1998; Manstead \& Parker, 1995). Until then, the nature of the control construct and its role in behavioral causation will be surrounded by ambiguities.

\section{Measuring Intentions}

The troubles of EV models in modeling the influence of control-perceptions over nonvolitional factors may, in part, be caused by inconsistencies conceming the assessment of the intention construct itself. Indeed, a final group of critiques targets the operational definitions employed to tap the most proximal determinant of behavior in current EV models (see: Armitage \& Conner, 2001; Eagly \& Chaiken, 1993, for overviews). In EV models, behavioral intention indexes a person's motivation to perform a particular behavior. In the TPB, intentions are quite loosely defined as beliefs about whether one will engage in a behavior (Ajzen \& Fishbein, 1980). Consequently, questionnaire items assessing intentions have been variably framed (cf. Sheppard, Hartwick \& Warshaw, 1988), either in terms of plans to 
engage in action (e.g. "I intend to perform X") or in terms of self-predictions or behavioral expectations (eg. "How likely is it that you will perform X"). Ajzen and Fishbein (1980) suggested that both types of measures could be used interchangeably.

This statement, however, has been questioned, as intentions, operationalized as behavioral expectations, may be more prone to inconporate implicit estimates of perceived control than intentions measured as plans Indeed, meta-analysis shows behavior to be better predicted by expectations than by plans (Sheppard, Hardwick \& Warshaw, 1988), undersconing the additional explained vaniance that comes from incorporating measures of control. A related, yet more pervasive challenge to either measure, however, states that precisely because behavioral expectations, as well as plans, tap control beliefs, the measures are rendered ambiguous (Smith, 1999). Nor expectations nor plans conceming for example, the use of soft drugs, distinguish between a person who wants to use soft drugs, intends not to, but expects to cave to desire or social pressure, on the one hand, and the person who wants to, intends to, and thus predicts to do so. Note that inconsistencies concerning the role of perceived control may thus stem from variation and potential flaws in the measurement of intentions.

Other concems, beyond ambiguities in operationalization, have been voiced with respect to the assessment of intentions. The extent, to which individual ratings of questionnaire items yield an accurate representation of a person's motivation to engage in action, may be limited for a number of reasons. First; tests of EV models do not usually encompass an objective measure of behavior, which renders intention ratings rather provisional (Sutton, 1998). In other words, intentions may be scored without any sense of obligation on behalf of the respondent, which makes them especially vulnerable to distortion by self-deception or social-desirability biases (Schwarz, 1999) and attenuates their value as predictors of actual behavior.

Secondly, it may be hard for participants to take into account the situational circumstances that affect their decisions. For instance, when expressing intentions to use a condom during their next sexual encounter, people may overlook or underestimate the influence of complicating factors, like the amount of alcohol they might have consumed or the attractiveness of their partner. Underscoring the importance of these situational influences, Gibbons and colleagues (1998) argued that a non-intentional component is involved in decisions, particularly to engage in risky or other actions with delayed-costs. Decisions to engage in actions like having unprotected sex, to drink and drive, or use soft drugs, should be considered "reactive" (ie., to arise in response to situational influences), rather than deliberative (i.e, to result from premeditated consideration). The concept of "willingness" was proposed as an additional predictor (next to intentions), in order to capture the reactive nature of these behaviors (Gibbons, Gerrard, Blanton, \& Russell, 1998). In assessing behavioral willingness, risk-conducive circumstances are first described to respondents before they are asked how they will react (in terms of a particular behavior) if they were in such a situation. For instance, respondents may be asked how likely they would be to: accept / say no / leave it to the situation, if a friend offered them a cigarette. Although 
empirical data supports the independent contribution of the willingness construct to predicting behavior next to intentions (Gibbons et $\mathrm{al}_{\mathrm{s}}$ 1998), one may question its potential to reliably capture the relevant situational influences, and whether it is possible for a respondent to accurately estimate the impact thereof. Nevertheless, many authors have stressed the importance of paying more attention to the way in which intentions may be modified by situational influences and advocated the development of measures that reliably capture and predict such influences (e.g, Sutton, 1998).

Finally, it has been argued that other psychological processes, beside the formation of intentions, may affect behavior. Unlike the contributions described in the present section, the existence of factors whose impact is not mediated by intentions, assumes the operation of psychological processes distinct or absent from those described in current EV models. They will therefore be considered in the next paragraph To first conclude the present discussion, however, it is safe to say that the intention-concept as yet remains underdeveloped in EV models (c. Eagly \& Chaiken, 1993).

\section{Beyond the Variance Explained...}

Although each of the modifications described above is - to a greater or lesser extent supported by empirical evidence indicating that the concept in question significantly improves the predictive validity of a given model, this approach to model improvement is also subjected to criticism. The common element in these comments concems the acknowledgement of the negative side effects of a narrow emphasis on explained variance as criterion for the quality of a model. In this section, three lines of argumentation will be developed to indicate that the percentage of variance explained may be an (1) ambivalent, (2) ambiguous, and even (3) a trivial standard of quality.

To start with the former, gains in explained variance need not necessarily imply model improvement. If indeed a new concept can be theoretically and empirically distinguished from others, it may be useful to incorporate it in an existing model As Ajzen (1991, p. 199) acknowledged, "The TPB is, in principle, open to indusion of additional predictors if it can be shown that they capture a significant proportion of the variance in intention or behavior after the theory's current variables have been taken into account:" Nevertheless, one of the advantages of models like the TPB is their parsimonious account of behavioral determinants and inclusions of additional variables with the purpose of increasing predictive validity should not be made too eagerly at the expense thereof (cf. Conner \& Armitage, 1998). Inconsistencies in the measurement and conceptualization of certain constructs still require settlement before the actual contribution of adding the variable to the model can be objectively determined.

Focusing on the percentage of explained variance has obviously (see the previous sections) resulted in an explosive proliferation of concepts, that are claimed to be distinct determinants of behavior, but in many instances share large conceptual similanities (Conner \& Armitage, 1998; Sutton, 1998). Furthermore, it has even spurred the development of numerous models that are merely distinguishable from one-another in their emphasis on 
one or more of these concepts (Weinstein, 1993). Such excessive differentiation is deemed to be one of the major causes of impediment to scientific progress as it causes fragmentation rather than integration of knowledge in a domain of inquiry (Kruglanski, 2001; Weinstein, 1993). Beyond that, conceptual incoherence has even been branded "Social psychology's ... most important and far reaching problem by far" (Markovsky, 1997, p. 25, emphasis in the oniginal):

Therefore, rather than continuing to expand models with an endless strain of "new" concepts, Abraham, et al, (1998) argue that it will be more fruitful to depart from a core EV model in which the general cognitive antecedents of behavior as well as vanious types of associated measures are specified. Such a model would provide a content-free framework that could subsequently guide identification and selection of concepts and measures in a problem-based fashion, whilst still acknowledging the common theoretical understanding of the cognitive antecedents of behavior.

Secondly, the criterion of explained variance has been defied for presenting an equivocal standard of quality: Consider the results of a study investigating a sample of 47 research articles explicitly incorporating structured EV models prominent in health psychology (Ogden, 2003). It was found that two-thirds of the studies in this sample failed to demonstrate significant predictive validity for one or more of the models' components and sometimes even for the model as a whole. Furthermore, the overall unexplained variance, ranged from $35-99 \%$ for behavior, and 8-86\% for intentions. Remarkably, the authors of these articles generally appeared reluctant to reject the modelor its assumptions and seemed more indined towards dismissing their data by claims of flawed methodology (Ogden, 2003; Weinstein, 1993).

To some extent this is understandable as achieving $30 \%$ of explained variance can incur both disappointment (as $70 \%$ is left unexplained) as well as satisfaction (as being able to reduce the likelihood of a behavior causing serious health risks is a considerable success in any case): Apparently, researchers seem inclined towards the latter. Furthermore, it has been noted repeatedly that the relative importance (iie the weights) of variables (like attitudes, subjective norms, and $\mathrm{PBC}$ in case of the TPB) for the prediction of intentions may vary (Ajzen \& Fishbein, 2004). In addition, as discussed, there may be several reasons that may bring about low predictive validity of the model in general.

However, these refutes do not appreciate the more subtle element of this critique. Articulating this; Ogden (2003, p.425) argued that: "All data can be used to indicate the strength of a social cognition model, but it would appear that no data can be collected to show that it is wrong. They therefore cannot be tested." The apparent reluctance or unfeasibility to challenge a model's validity by results (of cross-sectional studies) in terms of percentage of explained variance thus calls for additional standards of model testing, particularly experimental manipulations (e.g.; Sutton, 1998; Coner \& Armitage, 1998; Ogden, 2003; Swanbom, 1996): 
Thirdly and finally, the percentage of explained variance presents a rather trivial index of quality as it serves as a standard only for prediction, not for explanation. In order to appreciate the difference, consider the following example: An itching corn or a flock of lowflying swallows may be a very reliable predictor of tomorrow's weather conditions, signaling imminent stom. However, in order to understand why stomn is at hand, a com or swallows won't be of much use. Hence a meteorologist - whilst often not the best of forecasters - is nevertheless able to explain the apparent change in the weather in terms of air pressure etcetera. In a similar way, Sutton (1998) argues that the predictive qualities of a model (assessed by explained variance), should not be equated with its value as an explanatory tool for the phenomenon under investigation. A good explanatory model depicts the causal paths between several variables, and describes the processes by which each of them influences others. Of course, prediction without explanation can nevertheless be useful. In the field of health education, it enables interventions to be tailored to particular subsets of a population. However, theory-based health education in based on the assumption that a model portrays the causal influences through which certain factors influence our behavior. If not, changes in behavior that follow an intervention program cannot be attributed to the processes as described in the model, and theory-based health education based on such a model is but an illusion

No matter what opinion one holds regarding the predictive merits of EV models, the proposal that such correlations occur because of the causal impact antecedent cognitions have on behavior, is another issue that requires careful evaluation (Conner \& Amitage, 1998; Eagly \& Chaiken, 1993; Sutton, 1998; Weinstein, 1993). This will be the topic of discussion in the subsequent section.

\section{Model Refinement: Specifying the Causal Processes}

We ended the previous section by discussing the difference between prediction and explanation. The relationships between cognitions and behavior as specified in EV models could be regarded as merely predictive, having no implications for psychological processes. This narrow interpretation, however, would neither stroke with extensive theoretical discussion of beliefs as determinants of behavior in EV theory (e.g, Feather, 1982; Eagly \& Chaiken, 1993), nor with practical applications of such models. For example in the field of health education, model variables are held to be potential targets for interventions aimed to induce behavioral change, hence, to constitute behavioral determinants (eg., Kok, Schaalma, De Vries, Parcel, \& Paulussen, 1996). Indeed, in explicating their model, Ajzen and Fishbein (e.g., 1980) have frequently argued that beliefs are causally linked, through attitudes and intentions; to behavior.

In the present paragraph, however, we will address empirical evidence and theoretical contemplations that provide reasonable grounds to question the tenability of that daim. 
Whereas the revisions treated in the previous paragraph still fell within the reach of psychological processes underlying the reasoned-action perspective (eg, the deliberation of consequences of engaging in action), the contributions addressed hereafter either invoke additional processes of an altogether different nature, or at least, require further specification of processes already presupposed by existing EV models.

In the following section we will first evaluate the status of intention formation as the proximal determinant of behavior. Subsequently, the discussion will concentrate on the causal influence of beliefs on subsequent variables:

\section{Between Intentions and Behavior}

According to the reasoned-action perspective, behavior is ultimately caused by volitional choice (ie, an intention) to either act or not Nevertheless, EV models have often been criticized for providing quite a poor description of the processes that mediate between the formation of an intention and the (persistent) execution of behavior (e.g. Conner \& Amitage, 1998; Eagly \& Chaiken, 1993). Recapitulate from the previous paragraph that the association between both is generally found to be relatively weak. On average, intentions only predict about $20-30 \%$ of variance in behavior. Attempts to account for this so-called "intentionbehavior gap" ( $c$. Sheeran, 2002) have either studied (1) factors that influence the strength of the relation between intentions and behavior, or (2) other mechanisms that generate behavior, apart from volitional choice.

\section{Determinants of intention - behavior consistency}

Pertaining to the first category, we find three (often overlapping) lines of research. The first centers on stage models of behavior change, the second investigates moderators of intentionbehavior relations, and the third explores the contributions of goal-constructs. Each will be discussed in turn.

One potential cause of low intention - behavior consistency may arise from the fact that behavior is often not the result of a one-time decision but involves repeated and consistent choice on numerous subsequent occasions. For instance, Sheeran and colleagues investigated the relation between intentions to attend health screening sessions and actual attendance at two instances, one and twelve months thereafter (Sheeran, Conner, \& Noman, 2001). They found that, although intention predicted the frequency of attendance, it could not discriminate among people who consistently attended check-ups, those who delayed attendance, and those who did not maintain attendance. The authors concluded that intention measures were not able to predict behavioral patterns in actions that must be performed promptly and repeatedly. Several other models have been proposed, however, to explicitly account for differences in patterns of behavioral change, identifying discrete stages of preparedness in the enactment of behavior. Among those we find, for instance, the Transtheoretical model (Prochaska, DiClemente, \& Norcross, 1992) and the Rubicon model of action phases (Gollwitzer, Heckhausen \& Ratajczak, 1990). According to such models, people pass through several stages before a particular behavior is consequently adopted. Different 
determinanits are supposed to invoke the transition of one stage to another. Furthermore, these models identify different processes that underlie this progress. As such, stage models of behavior change may complement current EV models by increasing our understanding of differences in action initiation and maintenance. Nevertheless, evidence to support some of the central claims of such models is still sparse (eg., De Vet, de Nooijer, de Vries \& Brug, 2005; Weinstein, Rothman \& Sutton, 1998).

A second class of explanations accounting for low intention - behavior consistency comes from studies that try to identify potential moderators of the intention-behavior relationship. A moderator variable affects the relationship between two other variables (Baron \& Kenny, 1986). Hence, even if two people have an equally strong intention (e.g, as assessed by scale ratings), other properties, beside its intensity, may affect the extent to which an intention affects behavior. Recently, researchers have been investigating the impact of temporal stability of intentions as a moderator of intention-behavior relations (see: Cooke \& Sheeran, 2004, for a review). Remember that according to Ajzen and Fishbein (1980), intentions have to be measured in close temporal contiguity in order to be reliable predictors of behavior, for the longer the intervening time interval, the greater the chances that one's intentions may have changed. The temporal stability of intentions is thus believed to decline as a function of the time elapsed between the measurement of intention and behavior. Nevertheless, recent studies have indicated that temporal stability of intentions (computed from within-participants conrelations between intentions assessed at two time points) can moderate intention-behavior relations even after a 6-year time-period had elapsed since the temporal stability had been measured (Conner, Norman, \& Bell, 2002). This implies that temporal stability is a variable property of intentions rather than a mere indicator of the time elapsed between two measurements, and therefore presents a mechanism through which other factors influence the strength of intention behavior relations. Of course, research still needs to address what determines the temporal stability of intentions.

The third branch of research investigating determinants of intention - behavior consistency is founded on the idea that considering the goal-directed nature of specific actions will improve behavioral predictions (see: Abraham \& Sheeran, 2003; Gollwitzer \& Moskowitz, 1996, for reviews). Departing from the notion that the goals underlying human behavior are organized in complex hierarchies of varying levels of specificity (e.g., Carver \& Scheier, 1998), studies have attempted to relate specific actions to more general goals embedded in such hierarchies (Bagozzi, Bergami, \& Leone, 2003). For instance, two discrete actions (e.g. dieting and going to the gym) can be complementary with respect to a single goal (e.g. "loosing weight"). The goal to loose weight, in turn, may be an expression of a broader, higher order goal, such as "being healthy".

Goal-content theonies present a first subset of goal-based approaches. Generally, they try to specify how the effectiveness of execution of intended actions relates to differences in the goal that is being pursued. Research in this area has showed, for instance, that goal- 
specificity affects persistence in goal striving and general goal-attainment (Locke \& Latham, 1990; Vallacher \& Wegner, 1998): Specifically, formulating personal goals in vague terms (e:g, to do your best) appears to be less effective than holding more concretely specified goals (e.g., to finish the paper you're working on before the end of the monthl. Other factors that are found to boost effort, persistence, and achievement of goals are the goal-standard (challenging vs. modest), and the specified time frame for attainment (proximal- vs. distal, e.g. Bandura, 2001). Also, differences can be derived from regulatory-focus theory (Higgins, 1997), which investigates the impact of positively (eg. I want to be popular) versus negatively (I don't want to be unpopular) framed goals on performance. Furthermore, according to self-determination theory (Ryan \& Deci, 2000), goals that are pursued in the service of autonomy, competence, and social integration (i.e., intrinsically motivated goals) lead to better performance than extrinsically motivated goals that are imposed by authority or pursued in exchange for monetary incentives.

Self-regulation theories of goal striving, on the other hand, are concerned with the volitional processes that mediate the influence of goals on behavior (see: Karoly, 1993, for an overview). They investigate self-regulatory (i.e. willful) strategies that help the person overcome obstades in goal implementation, eg., to ward of distractions and negotiate conflicts between goals. The formation of implementation intentions (Gollwitzer, 1999), for instance, constitutes a self-regulatory strategy to overcome problems in initiation of goal directed behavior. Whereas an ordinary measure of intention constitutes a statement like, I intend to do $\mathrm{X}$ ', an implementation intention requires the respondent to denote the time and place where the intention will be enacted. For instance, I intend to perform a testicular selfexamination every Saturday while I'm in the shower'. An impressive body of evidence indicates that specifying such implementation intentions increases both the likelihood and the immediacy of action initiation (see: Sheeran, 2002 for a review). Additionally, several researchers have addressed sources of individual or situational differences in self-regulation processes. For example, it has been demonstrated that individual differences in selfregulation may arise because of differences in information processing (Kuhl \& Beckman, 1994). Kuhl distinguishes between "action" and "state oriented" individuals. The latter group in particular appears to experience troubles in translating intentions into actions, probably because of a tendency to become occupied by failure. Furthermore, research on "egodepletion" indicates that the capacity for self-control is limited (Baumeister, Bratslavsky, Muraven, \& Tice, 1998). Basically, these studies suggest that task endurance and performance decreases after subjects were previously asked to perform another task that required them to exert self-control (e.g, suppress temptations or the expression of emotions). Such ego-depletion effects may account for prevalent relapses to unhealthy behaviors (like smoking) after mentally or emotionally upsetting events. Research on goal-setting and selfregulatory processes presents an important contribution to EV models as it contributes to the identification of various processes that mediate the successful translation of intentions into behavior (Abraham \& Sheeran, 2003; Abraham et al, 1998). 


\section{Automatic processes and habitual action}

Within the three lines of research presented above, intention formation is still considered the proximal cause of behavior. Nevertheless, an influential field of research has namrowed the scope of volitional control mechanisms by indicating that in many instances, behavior is not the result of a conscious decision, but is generated by automatic (ie, unconscious and unintentional) processes.

This research stems from the observation that often, the frequency with which a behavior has been performed in the past is found to be a unique (if not the best) predictor of later actions, independent from standard EV variables like intention and perceived control (see: Eagly \& Chaiken, 1993, for a review). Clearly, past behavior does not cause future behavior much like yesterdays weather does not cause tomorrows weather but may still be a reliable indicator thereof. This is exactly the argument that has prevented researchers to attribute any importance to the possible influence of past behavior for a long time.

Nevertheless, accumulating evidence shows how environmental stimuli may automatically (i.e, in a non-conscious manner) affect people's judgment, decisions and behavior (for reviews; see: Bargh \& Chartrand, 1999; Bargh \& Gollwitzer, 1994). For instance, implicitly (that is, without the person being aware of it) activating an achievement goal increased the effort as well as the performance of participants in solving puzzle tasks. According to the auto-motive model (Bargh, 1990), goals can become activated unconsciously by environmental cues and may subsequently instigate behavior exactly like consciously intended ones do, without necessitating an individual to make an intentional decision to perform the act. Underlying this capacity is the process by which direct situation-to-goal links develop over the course of time from consistent and frequent conscious choice of a particular goal and subsequent action in a given situation According to the model, this intentional choice ultimately becomes automated in much the same way as occurs during skill acquisition (eg., learning to drive a car).

Mirroring these presumptions, the results of a meta-analysis on the predictive value of past on later behavior indicated that especially in case of actions that were frequently performed under constant conditions (such as the use of seat belts, smoking, sporting), future behavior was reliably predicted by past behavior (Ouellette \& Wood, 1998). Behaviors that were performed only sporadically, however, were better predicted from intentions. These results support conceptualizations of the relation between past and future behavior in terms of "habits", defined as acquired, automatic, and gaal-directed responses to environmental stimuli, as described by the auto-motive model In this sense, habits present a mechanism granting psychological significance to the association between past and future behavior as causal account of goal-directed action, next to intention formation (Aarts, Verplanken \& van Knippenberg, 1998; Conner \& Armitage, 1998).

Nevertheless, difficulties still surround the conceptualization of habits, which is due to their operationalizations in terms of the frequency of past behavior (Ajzen, 2002b). For instance, even though I've dimbed Mount Everest a dozen times now, this still hasn't become 
habitual to me. Researchers have attempted to develop different measures, however, that more objectively assess whether a particular behavior constitutes a habit. For instance, the response-frequency measure assesses habit strength indirectly, based on response latencies of choices between response altematives (e.g., Verplanken \& Aarts, 1999). For instance, in order to measure people's habit-strength for a particular mode of transportation, participants were asked to choose as quickly as possible between several altematives (e.g, bike, car, bus) for going to a number of different locations (to the bar, a friends' place, or school). Habit strength to use the bike was determined by the number of times and the speed with which people chose this as their first option.

Using such measures, subsequent studies (e.g, Aarts \& Dijksterhuis, 2000; Aarts et al, 1998) have started to elucidate the processes through which habits influence future behavior. Results indicating that habit strength moderates the intention - behavior relation (iie, the stronger the habit, the less well behavior can be predicted from intentions) provide support for the view that intentional and automatic processes determine behavior in concert. Furthermore, it appeared that people with a strong habit for a particular behavior, engaged in less extensive searches for information before making a behavioral decision. This provides further support for the idea that habits are automatic responses to particular situational cues. When confronted with this situation a particular choice becomes immediately salient to people with a strong habit.

The existence of unconscious processes controlling our actions restricts the scope of health interventions based on persuasion and communication that attempt to influence conscious, intentional processes. However, further research is still required to determine the generality of automatic processes. The impact of automatic goal-priming effects, for instance, on the sort of behaviors that are of interest to applied researchers (e.g, health-related behaviors), still awaits empinical demonstration (Sheeran, 2002).

Volitional choice constitutes the final step in the causal chain of action generation as portrayed by the in the reasoned-action perspective The research discussed in the present section, however, demonstrates that many blanks still need to be filled in before a comprehensive understanding is reached of the psychological processes involved in action initiation and maintenance. In the subsequent section, we will move further up the causal chain of the reasoned-action perspective and take a closer look at the process ultimately underlying the formation of intentions; the deliberation of possible consequences of action.

\section{From Beliefs to Actions}

According to the standard EV model, the causal impact of cognitions on behavior (and consequently, on intermediate variables) can be described as a function of the sum of the expected values of relevant attributes (Feather, 1982; Eagly \& Chaiken, 1993). For instance, in case of EV modeling of behavior as depicted in the TPB, one's subjective probability estimate that a given behavior results in a particular outcome (the expectancy component) is multiplied by one's (positive or negative) evaluation of that outcome (the value component), 
and this product is subsequently summated with that of all other salient outcome beliefs. (OF course, EV models do not assume people to actually engage in this type of cognitive algebra while making a decision, but merely attest that people behave as if they do). The adequacy of expectancy-value products for modeling the impact of beliefs has not been unchallenged, however. In the present paragraph, we will discuss theoretical and methodological issues in testing the causal influence of beliefs on behavior, starting with the latter.

\section{Methodological complications}

First of all, several methodological concerns have been raised that; not so much as questioning the causal impact of cognitions per se, undercut the feasibility of empirically testing the influence of EV-scores on a dependent variable. One problem constitutes the widespread - yet improper - way of statistically analyzing models containing multiplicative terms (like expectancy-values), which persists in spite of repeated warning (eg. Evans, 1991). The trouble in testing such relations arises due to the fact that correlations between multiplicative terms and another variable are not invariant to otherwise acceptable transformations of the scale. A study investigating the impact of such transformations, assessed EV-products rated on a seven-point scale that was either coded from -3 to +3 or from 1 to 7 (Schmidt, 1973). Results revealed that the first scale correlated .30 with the dependent variable, whereas the latter correlated 46 . It should be noted that only the numbers that the investigator assigned to the answers were changed, not the question or response format that was presented to the participants. Apparently, one cannot be confident about the effect size for simple correlations involving multiplicative composites (Evans, 1991). Essentially, assessing relations with a multiplicative composite is tantamount to assuming an interaction and requires initial controlling of the separate effects of the two components (Baron \& Kenny, 1986). Such analysis, however, often places impracticable requirements on sample size and reliability of measures (Evans, 1991).

Results demonstrating that attitudes may just as reliably be predicted from additive models of expectancies and evaluations separately, ostensibly present an opportunity to sidestep the problems surrounding the analysis of multiplicative terms (eg. Trafimow \& Finlay, 2002). Closer examination of the meaning of the additive model, however, does not hold in the face of logical interpretation, as neither the expectancy, nor the value term makes sense as a single predictor (Eagly \& Chaiken, 1993; Swanbom, 1996). My perception of eating raw broccoli as extremely effective in reducing weight, for instance, has no necessary implications for determining my attitude. (What if, for example, I'm a sumo wrestler preparing for a big sumo-wrestling toumament?) Neither does a positive evaluation of loosing weight need to have any implications for me eating raw broccoli; if I do not attribute this particular property to this particular vegetable.

Other methodological problems concern the unipolar of bipolar rating of outcome expectancies. Essentially, bipolar rating enables disbelief in negative attributes to contribute positively to the composite (cf. Trafimow \& Finlay, 2002). For instance, perceiving it as very unlikely $(-3)$ that eating raw broccoli will cause stomachaches whilst negatively evaluating 
(-3) stomachaches, would provide an impetus ( +9$)$ for eating raw broccoli Framing the same belief in the opposite direction (eating raw broccoli will not cause stomachaches) would make no difference according to the basic assumption of the EV model Framed as such, it would be considered extremely likely $(+3)$ and evaluated extremely positively $(+3)$, hence have the same overall impact when it comes to predicting one's global attitude towards eating raw broccoli. Experimental testing, however, revealed that beliefs framed in terms of positive consequences to be better predictors of attitude than negative framed beliefs (Trafimow \& Finlay, 2002), which cannot be explained by any of the assumptions underlying EV accounts of the impact of cognitions.

Others argue that beliefs should be measured using one-sided scales, ranging from zero to some maximum value (e.g., Hewstone \& Young, 1988; Smith, 1996). This, nevertheless, appears to reduce the correlation of EV-scores with the overall attitude measures (Sparks, Hedderley, \& Shepherd, 1991). Ultimately, the best solution to this methodological conundrum would be to assess only individually salient beliefs and respondents' evaluations thereof as the single predictor of attitudes, as originally proposed (Ajzen \& Fishbein, 1980).

\section{The direction of causation}

A somewhat more fundamental issue concems difficulties in unequivocally establishing the proposed causal influence of cognitions on behavior or intermediate variables. This problem is twofold, having both a methodological and a theoretical component. Pertaining to methodology, some authors have pointed out that the common practice in studies of EV models, that has participants completing a questionnaire to describe their cognitions, relies on the assumption that the answers given will reveal preexisting states of mind. Nevertheless, it could be possible that filling out a questionnaire creates, rather than assesses cognitions (Ogden, 2003). This is particularly likely to occur in case the behavior under investigation is novel and unfamiliar or in case the presented consequences are unexpected or unknown. For instance, presenting respondents with a list of possible attributes of alien topics like female condom use, new food products, bone marrow donation, etcetera, seems unlikely to tap existing beliefs, as opposed to inducing new ones. Results from research on implementation intentions and anticipated affect seem to corroborate such suspicions. Evidence from both lines of research suggests that asking respondents certain questions (ite, to phrase their intentions in terms of action plans or to consider their emotional reactions to particular outcomes) has the potential to bring about behavioral change. These results therefore demonstrate that question asking can induce changes, and it may be hard to safely decide when the same process of question asking and answering is descriptive and when it will be interventional (Ogden, 2003). It may, of course, be relatively easy to evade this problem, for instance by counterbalancing the order of questionnaire items measuring attitudes and beliefs, or by using a free elicitation format (Ajzen \& Fishbein, 2004). Nevertheless, it seems researchers are not always aware of this issue when designing questionnaires. 
On a theoretical level, ambiguities concerning the impact of beliefs may anise as causal influences can flow from beliefs to a supposed outcome variable as readily as the other way around (Eagly \& Chaiken, 1993). For example, a person who has a positive attitude towards Amold Schwarzenegger because of his excellent robot performances does not necessarily need to have considered whether he is politically aware, socially involved, and a good leader. Yet when checking hüs ballot for the govemmental elections of Califomia, this person might have voted for him because it would have seemed plausible that such a fine actor possesses these other qualities as well (After all, "The Terminator" has already saved the world twice.) Such instances of reciprocal causation are not limited to relations between attitudes and beliefs: Similar feedback effects have been reported for the association between attitudes and behavior (Eagly \& Chaiken, 1993). For instance, attitudes may detemine subsequent behavior as one may decide to go and see the new Amold Schwarzenegger movie because he's such a fine actor, but attitudes may also be inferred from behavior through selfperception processes (e.g.; "T've seen all his movies, so I guess I think Amold is a great actor"). Whether the former or the latter occurs appears to be determined by the strength of an individual's attitude. Results from a study by Holland et al indicated that strong attitudes (towards Greenpeace) were better predictors of (donating) behavior assessed one week later than weak attitudes (Holland, Verplanken \& van Knippenberg, 2002). Moreover, attitudes towards the work of Greenpeace, assessed after the behavioral measure, were related to previous behavior for people with weak attitudes but not for peopie with strong attitudes. The authors concluded that strong attitudes guide behavior, whereas weak attitudes are inferred thereof (Holland, et al, 2002). Similarly; research on cognitive dissonance has repeatedly indicated that attitudes are brought in line with actions performed. It should be noted that EV' models do not assume such feedback-effects between variables. Therefore, the direction of the causal flow between variables like beliefs and attitudes, rather than being a justified assumption, is an empirical question that underscores the need for experimental testing or at least to implement longitudinal designs in $\mathrm{EV}$ research (Sutton, 2002a).

\section{Questions about the causal nature of beliefs}

There are certain other aspects - beside the bidirectional nature of their relations - of the causal link between beliefs and attitudes that cannot be explained by, or at least require further specification in, current EV models.

First of all, EV models assume that a person may hold a large number of beliefs concerning the attributes of a particular attitude object, but that given the constraints of the human capacity for information processing, at any moment, only alimited number of these beliefs is salient, which determine the individual's attitude. Acknowledging these limitations of the capacity to process and store information, Fishbein and Ajzen (1975) suggested that, as a rule of thumb, the first five to nine beliefs to be expressed in a free elicitation procedure, constitute an individual's salient set. Unfortunately, this idea did not consistently perspire into measurement, and in practice, respondents are usually presented with a modally salient set of attributes that is derived from an average population, which may not adequately 
capture the salient beliefs of an individual (van der Pligt \& Eiser, 1984). Setting out to develop attribute based measures of attitudes that match human information processing capabilities, van der Pligt and colleague's performed a series of studies investigating the possibilities of assessing belief salience by explicitly asking respondents to rank or rate the relative importance of each belief in determining their overall attitude (see: van der Pligt, de Vries, Manstead, \& van Harreveld, 2000, for an overview). Although Ajzen and Fishbein originally asserted that such importance scores would be closely related to either the evaluative extremity or the perceived likelihood of beliefs, results indicated that although correlations between importance rating and EV scores were generally significant, their magnitude did not allow them to be considered redundant. Further studies indicated that belief-attitude correlations with a subset of the three most salient beliefs were even stronger than with all modally salient beliefs combined (van der Pligt \& de Vries, 1998a). Also, experiments showed shorter response latencies for evaluating salient versus non-salient beliefs, which accounts for the greater impact of important attributes on overall judgments (van Harreveld, van der Pligt, de Vries, \& Andreas, 2000). Finally, it tumed out that different beliefs were important to different groups (e.g. smokers and non-smokers) with respect to a particular behavior (van der Pligt et al, 2000). Based on these findings it is argued that measures of belief importance should be incorporated in EV models, as this would increase the descriptive validity as well as the practical applicability of such models in informing the design of interventions to change behavior (Conner \& Armitage, 1998).

Notwithstanding this proposition, it seems to have gone largely unnoticed that the possibility of some beliefs being more important causes of attitudes than others, conflicts with one of the key theoretical assumption of basic EV models; namely, that all attributes have equal weights in determining the outcome variable under investigation (Sutton, $2002 \mathrm{~b}$ ). The results described above, therefore, involke the more fundamental question of why some beliefs are more important than others or more important to different groups of people. Hence, whether the weights have psychological meaning and if so, what would be the nature of the underlying process(es).

Current EV models remain silent on questions such as the one mentioned above as they fail to specify the nature of beliefs. It is sometimes even argued that - beyond the issues of bidirectional influence and differential weights - it remains unclear why beliefs, as specified in current EV models, would have causal properties altogether (e.g, Abraham \& Sheeran, 2003; Bagozzi, 1992; Balleine \& Dickinson, 1998; Frijda, Manstead, \& Bem, 2000; Gollwitzer \& Moskowitz, 1996; Hanoch, 2002; Pervin, 1989). Specifically, there is nothing about a belief, defined as a (positively or negatively evaluated) expected outcome of behavior that would necessarily instigate the person holding it to act accordingly. In other words, beliefs, in themselves lack the motivational potential needed to induce an action, ie, they are motivationally vacuous. Frijda (e.g., Frijda et al, 2000) illustrates this point by refering to the common sense observation that in everyday life, most people hold a great deal of beliefs conceming positively valued consequences of many possible actions (e.g, donating money to 
Unicef, separating ones' garbage, excrcising regularly), to which we nevertheless require some impetus before we actually perform them (like shocking television images of people in need, a documentary on global warming, or pants that no longer fit).

This problem is manifested in the general consensus on the position that too many variables of interest to social psychologists are discarded as extraneous variables by cument EV models and in particular by the TPB (Abraham \& Sheeran, 2003; Eagly \& Chaiken, 1993; Swanbom, 1996). According to Ajzen and Fishbein (1980), other variables than those specified in their model may have an impact on behavior, but orly through influencing either attitudinal or subjective norm beliefs. Consequently, the model does neither specify the nature of these distal variables, nor indicates the way in which they affect beliefs. Most of the ambiguities concerning the causal influence of beliefs as discussed so far in the present section, may be attributed to this silence regarding more distal factors.

Several authors (eg., Bagozzi, 1992; Abraham \& Sheeran, 2003; Pervin, 1989) have argued that integrating EV models with goal theories may help, not only to elucidate when and how intentions will result in the successful enactment of behavior as outlined in the previous section, but also to indicate the motivational nature of beliefs that would explain how behavior becomes energized. However, few attempts have been made to structurally integrate EV models with concepts derived from goal theories, and, to our opinion, neither of those is able to satisfactory account for the goal-related nature of the system of beliefs that underlies the formation of intentions: In the following, three examples will be discussed of research integrating EV models with goal concepts that illustrate the flaws in current attempts to relate beliefs to goals.

In the Model of Goal-Directed Behavior (MGB), Bagozzi (1992; Perugini \& Bagozzi, 2001) proposes that desires constitute the link between an intention and a general goal underlying the behavior. Desires are defined as the motivational state of mind wherein appraisals and reasons to act are transformed into motivation to do so. According to the MGB, an intention, for instance to take up a diet, ensues from a desire to do so. The desire, in tum, could be the result of a goal to loose weight. Desires arise from anticipated affective consequences of goal achieverment or failure. Hence, the anticipation of disappointment from staying fat or the anticipated joy from looking nice in swimming dothes again. The extremity of the anticipated affective reactions signals the extent to which individuals are motivated to achieve the goal underlying the behavior, hence determines their motivation to do so. Both desires and anticipated affect, however; are introduced as separate variables into the model, next to attitudes, subjective nom, and PBC. They therefore do not help in elucidating the goal-related nature of the cognitive antecedents to these variables.

Other contributions do pay specific attention to the influence of goals on EV variables. For instance, a factor called goal-importance was proposed as underlying all other variables in EV models (Sideridis \& Kaissidis-Rodafinos; 2001). The results of this study indicated that both intentions and attitudes (towards "studying hard the next four weeks") could be reliably predicted from the importance of the goal to do so, which was assessed by items like: "The 
highest prionity for me is to study hard for four weeks". Although the goal construct was integrated into the EV model in this study, it was conceptualized so specifically with respect to the behavior under investigation, that a relation with any of the outcome expectancies (e.g." "Studying hard will improve my job opportunities") was lost. Furthemore, by specifying goals almost at the same level of specificity as the behavioral act itself; it can hardly be argued that results like this contribute to elucidating the general goal-related origin of EV constructs.

More global goal constructs were specifically addressed in other studies, however. For example, it was suggested that incorporating general motivational orientations derived from self-detemination theory (Ryan \& Deci, 2000) may help to clarify the goal-related nature of attitudinal and subjective norm related cognitions (Hagger, Chatzisarantis, \& Biddle, 2002). Assessing the extent, to which individuals are extrinsically or intrinsically motivated to perform a specific behavior, appeared to determine the relative impact of attitudinal and normative components on intentions. Intrinsically motivated people engage in action for personal enjoyment, whereas extrinsically motivated people engage in action because of external reinforcement (ie, rewards or punishments). As attitudes are determined by beliefs conceming the personal consequences of behavior (e.g., dieting is good for my health), intentions of intrinsically motivated people are better predicted from their attitudes. Subjective norm, on the other hand, is composed of anticipated reactions from other people (e.g. my partner wants me to take up a diet), and consequently, mediates the impact of extrinsically motivated people.

Whereas this approach is more specifically concemed with the impact of broad goal constructs on the antecedent cognitions of EV models, the motivational influences described are of a non-directional nature. Several non-directional motivations are identified in the social cognition literature (e.g., Kruglanski, 1999), like accuracy motivation, need for closure, and the need for cognition. Although related to important differences in reasoning and judgment, such motives have no directive impact conceming the execution of behavior, nor do they specify the impact of specific beliefs. For instance, the same behavioral act (e.g., reading a research article) may be just as readily executed by an intrinsically motivated person (reading out of curiosity) as an extrinsically motivated individual (reading to study for an exam). Neither will these motivational onientations elucidate the origin of specific outcome expectancies (e.g. If I read this article I will: "Pass my exam" or "Miss tonight's party").

To summarize, current models that incorporate both EV and goal-related variables encounter difficulties in specifying how goals relate to particular outcome expectancies. In some instances (e.g., Perugini \& Bagozzi, 2001) goals are incorporated as additional variables and their association with EV variables - apart from intentions - remain unspecified. In case goal constructs are conceptualized as undertying constructs, they are either conceptualized too namowly; almost at the level of behavior (e.g., Sidenidis \& Kaissidis-Rodafinos, 2001), or non-directional, for instance in terms of intrinsic or extrinsic motivation (e.g, Hagger, et al, 2002). The problem with the former is that they remain context specific and will not account 
for differences in the execution of behavior in general. Furthermore, they provide only a spurious solution to explaining differences in the impact of beliefs as the question remains why individual differences exist in the importance of these goals. Non-directional goal constructs on the other hand, may exceed specific situations and seem to derive from stable individual differences (Ryan \& Deci, 2000), but have no direct connection with either the content or the evaluation of specific beliefs.

\section{SUMMARY AND CONCIUSIONS}

According to Kurt lewin (1935), "there's nothing as practical as a good theory". Of course, the sixty-four-thousand-dollar question here is: "What constitutes a good theory?" Like so many things, this depends upon one's stance or interest. To an applied researcher in the field of health education, a good theory would be the one that is effective, either as a tool in developing interventions aimed at behavioral change, or as an instrument to evaluate them. The use of theories indeed appears to be an important determinant for success as theorybased interventions (i.e., based on models of health behavior) do appear more effective at inducing behavioral change (Kok, van den Bome, \& Dolan Mullen, 1997). This, however, tells us nothing about the actual effectiveness of these interventions (e.g, as compared to a control group).

\section{The Effectiveness of Interventions}

Conceming these matters, several important lessons could be learned from a recent review investigating applications of the TPB in behavior change interventions (Hardernan, et al, 2002). The review included 30 papers, describing 24 distinct interventions that all used one or more model variables to evaluate the effectiveness of the intervention, and half of which used the model to also design the intervention. It is worth mentioning that most studies were poorly designed (e.g., many did not employ a randomized control trial), applied nonvalidated measures, and mostly relied on measures of intention as a proxy for behavior.

As expected, the principal methods applied to induce change were cognition based, relying on the transmission of information and persuasive communication. About $50 \%$ of the interventions that were specifically based on the TPB reported significant change in intentions, and only $17 \%(n=4)$ actually indicated change in behavior. The effect sizes of these studies were generally of small to moderate magnitude. Finally, only two studies investigated whether change in intentions or behavior was also mediated by targeted TPB variables. Both found results in accordance with the model Based on these findings, the authors concluded that the: "Data ... provides little evidence that use of the TPB to develop the intervention is associated with change in intention and behavior in the desired direction." and that: "At present there is ... insufficient evidence to judge whether TPB components mediate changes in intentions and behavior within evaluated interventions." (Hardeman, et al, 2002, p. 149). 
Several factors unrelated to the validity of the model may influence the effectiveness of behavioral interventions (e.g, Kok, et al, 1997). Neventheless, based on the preceding survey of comments it seems quite naive to exclude this latter possibility as a plausible cause for the limited effectiveness of TPB based interventions. Besides, the use of models is not only an important prerequisite for the success of an intervention, but the success of an intervention is also an important indicator of the quality of a model (Abraham et al, 1998; Ajzen \& Fishbein, 2004; Armitage \& Christian, 2003).

In the present chapter we presented an overview of accumulated dissident reflections conceming EV models. This survey presented various explanations for the limited effectiveness of health interventions based on EV models as evident in the Hardeman study. First, failure to induce behavioral change by cognition-based interventions may be the result of low predictive validity of EV models (Sutton, 2002b). Indeed, a wide range of concepts has been proposed as additional variables to the standard EV model, which suggest that there is ample opportunity for improvement in this domain. Nevertheless, we discussed several reasons that warrant scrutiny regarding this approach to model improvement. Apart from the fact that there seems to be empirical ground to question the tenability and generalizability of some additions, ambiguities also follow from the notion that the percentage of variance explained can be both an ambiguous and, in some respects, a trivial standard of quality. The purported ambiguity ensues from the fact that a certain amount of variance explained, no matter how low, always provides some support for the model under investigation, and researchers therefore seem reluctant to discard data on the basis of this criterion (e.g., Ogden, 2003). More importantly, however, the percentage of explained variance, being an index of a model's predictive potential, has no bearing on its providing a valid account of the psychological processes under investigation (e.g., Sutton, 1998).

Indeed, EV models are criticized for providing a deficient description of the causal processes underlying behavior. Methodological difficulties in testing some of the models' assumptions aside, it is pointed out that the processes mediating the associations between intentions and behavior remain underspecified in most models (Abraham, et al, 1998; Armitage \& Christian, 2003; Eagly \& Chaiken, 1993; Sheeran, 2002). Moreover, given the evidence for automatic influences on behavior (e.g. Bargh \& Chartrand, 1999; Verplanken \& Aarts, 1999), the emphasis on intentional processes in the reasoned-action perspective may be too excessive anyhow. Also, the models have trouble accounting for the origin of individual or situational differences in the impact of beliefs (Sutton, 2002b) and even fail to specify the causal status of cognitions altogether (e.g., Bagozzi, 1992; Balleine \& Dickinson, 1998; Frijda, et al, 2000; Hanoch, 2002).

Obviously, less than optimal predictive power presents a profound restriction to the models' potential for practical application, but also provides opportunities for improvement, for example by incorporating additional constructs. However, if the causal processes are not accurately described, this renders the model vacuous as a tool for designing theory based 
behavior interventions. It is precisely therein, in the investigation of the tenability of a model's propositions and assumptions, where we find the merits of fundamental research.

\section{Cardinal Sins}

We believe that the discussion presented in this chapter justifies dissuading from several frequently observed practices in research on EV models. Though their consequences are unlikely to be as dire as their counterparts in daily conduct, they could be summarized with reference to the seven cardinal sins.

First of all, pride; the excessive belief in one's own abilities is considered to be the most fundamental sin, underlying all others. Pride would reside in persistently sticking to the reasoned-action perspective described in EV models as a comprehensive account of goaldirected behavior, as was originally put forward (Ajzen \& Fishbein, 1980). Abundant empirical evidence indicates that the account of behavior presented by EV models is neither sufficiently accurate (considering the procedural comments), nor complete (given the evidence for automatic processes as well as the various extensions proposed). But still, one should be careful to avoid gluttony, the desire to consume more than is warranted, expressed in the excessive expansion of the basic model with additional concepts that have yet to prove their merits (Abraham et al, 1998; Conner \& Armitage, 1998; Eagly \& Chailken, 1993), as well as envy, the desire for another's traits, status, or abilities. The latter is evident in unwarranted model differentiation based on summary differences, and discarding opportunities for integration (Weinstein, 1993). Perhaps underlying both is greed, the desire for material wealth or gain, avoiding the realms of the spiritual, which symbolizes the narrow emphasis on explained variance as the sole criterion for model improvement, neglecting aspects of causal validity (Sutton, 1998; Swanbom, 1996). Fifth, sloth, the avoidance of physical or spiritual work, is manifest in the frequent failure to test model assumptions experimentally (e.g, Conner \& Armitage, 1998; Hardeman, et al, 2003; Ogden, 1993; Sutton, 2002a; Swanbom, 1996). Sloth also characterizes the over reliance on measures of intentions when trying to predict behavior. Both practices are condemned by Marks (among others), who stated that: "The theory failed as an explanatory account of health behavior ... and the dependent variable preferred for its convenience by almost all investigators - behavioral intention - proved to be a notoriously poor predictor of health preventive action." (as cited in: Sutton, 1998, p. 8). Note that Marks uses the term 'convenience' to describe the excessive reliance on measures of intention. Indeed, one might wonder whether adopting a theory based approach in health education not only resulted in more systematic practices but also might have gone hand-in-hand with a certain overconfidence or even latent laziness where it comes to the evaluation of interventions, by relying on theoretically grounded, yet poor indices of real results (read: behavior change) such as intentions. Lust, the desire for sensuous gratification, refers to he hedonic principle that underlies EV accounts of behavior. According to the hedonic principle, behavior can be understood as attempts to approach pleasure and avoid pain. Though not ultimately incorrect, Higgins (1997) argued that the notion that people approach pleasure and avoid pain presents too shallow an account of human motivation For one, the hedonic principle cannot account for the different ways in which 
people try to avoid pain and approach pleasure, ie., for the type of behaviors they engage in and the goals they thereby aim to achieve. As we shall see, the goals that people try to attain through their actions are more differentiated than can be captured by a single pleasure pain continuum. Furthermore, this may have important implications for the successful design of interventions.

Finally, however, we wish to emphasize that all comments aside, one should avoid to succumb to anger, the opting for fury, which would speak from failure to recognize the merits of the cognitive approach by adopting an overly critical attitude. One should be wary not to loose touch with the undeniable qualities of EV models, and again particularly the TPB, which constitute the extremely parsimonious account presented by these models and the wide range of behaviors to which they can be applied (Eagly \& Chaiken, 1993; Armitage \& Christian, 2003).

\section{Beyond Expectancy-Value Models...}

There may be various ways to proceed beyond the EV model as currently specified in dominant health-behavior models. Apart from the preceding caveats, we have - as stated no intention to judge the merits of any of these. In the final section of this chapter, we instead introduce our own proposal for improvement.

In our research, we focus on the deficiencies conceming the causal influence of beliefs on behavior as specified by current EV models. As advocated by several authors, we wish to determine the extent to which incorporating goal constructs may complement and elucidate the description of these processes by considering the goal related nature of beliefs. As argued, it is necessary therefore, to specify goal constructs at a sufficiently fundamental level to allow for generalizability across behaviors, yet still bearing evident associations with particular outcome expectancies. In the next chapter, we will start by proposing a way to adequately conceptualize goals to enable integration of both perspectives.

To end the present chapter, however, we must address Ajzen and Fishbein's original motivation for not considering the impact of distal variables (such as goals) in their model As stated, they held that such influences would affect behavior only through the variables specified in their model. Moreover, as no consistent predictions were likely to be made beforehand conceming the way distal variables influence behavior or intermediate variables, they saw no reason to specify them. Thirdly, they thought that different distal variables were required for different types of behavior, which would compromise the generalizability of their model across behaviors. Finally, they contented that: "the theory's validity depends not on support for hypotheses conceming the effects of external variables." (Ajzen \& Fishbein, 1980, p. 9).

The first claim has already been disconfirmed by research on automatic processes. In the remainder of this book we also hope to demonstrate that both the second and third provision are incorrect. Finally, although we do subscribe to the suggestion that external influences need not have implications for the theory's validity, we will argue that nevertheless, such influences may reveal previously unrecognized constraints in translating the reasoned-action perspective into practicable and effective behavior change interventions. 


\section{Chapter 3}

\section{$O_{n}$ the $O_{\text {rigin of }}$ Goals}

\section{and the Role of Emotions in the Control of Behavior}

\section{INTRODUCTION}

To recapitulate, in the previous chapter it was argued that the description of the processes underlying behavior as provided by current EV models is motivationally vacuous. Specifically, it was stipulated that nothing about an outcome expectancy (positively or negatively valued), necessitates the holder to act accordingly (e.g. Balleine \& Dickinson, 1998; Frijda, Manstead, \& Bem, 2000; Pervin, 1989). In other words, what is lacking in current EV models is the motivational drive that "energizes" behavior (cf. Bagozzi, 1992).

It is proposed that in order to account for the causal influence of outcome expectancies on behavior, it would be necessary to integrate EV models with goal-based approaches of behavior (e.g., Abraham \& Sheeran, 2003; Gollwitzer \& Moskowitz, 1996). Characteristically, goal-theories explain behavior by referring to needs and desires that guide human behavior (see: Austin \& Vancouver, 1996; Pittman, 1998 for overviews). We will addiress this approach as the Motivational Perspective on behavior.

Attempts are indeed being made to introduce goal concepts of varying nature into the standard EV model. We argued, however, that as yet they fail to provide a satisfactory description of the relation between goals and beliefs. Either because the goal is 
conceptualized too specific, ie, with reference to the behavior under investigation (e.g., the goal to loose weight). Such specific goals provide no insight into the motivational factors that underlie human behavior in general or because they do specify general motivational orientations, but conceptualized as non-directional motivations (cf. Kruglanski, 2001), for instance in terms of intrinsic vs. extrinsic motivation. Non-directional aspects of motivation, however, have no directional implication for behavior, as the same act (e.g, studying for an exam) can be intrinsically (studying out of curiosity) as well as extrinsically (studying to obtain a good grade) motivated.

As follows from the three examples mentioned above, in order to integrate EV models with goal-constructs, we first need to identify what general goals underlie behavior before we can specify how these goals are related to specific outcome expectancies. The latter will be outlined in the Chapter 4 . In the present chapter, we will be occupied by determining the contents of a taxonomy of general goals.

This chapter roughly consists of three parts. The first part deals with the requirements of a taxonomy of general goals. In the second part we suggest that emotions constitute the general goals of behavior, and the third part presents two empirical studies providing further support for our proposal to conceive of emotions as general goals.

The first part, briefly describes previous attempts to develop a taxonomy of general motives. We will argue that these attempts failed because researchers did not have a clear conception about the origin and hence, about the nature of the fundamental goal construct. Without such a conception, it is not possible to determine an objective criterion for identifying these goals. We will then propose that the general goals underlying human behavior should derive from evolutionary requirements imposed upon the behavior of organisms. Specifically, it will be argued that the general goals of behavior derive from adaptive problerns; opportunities to enhance fitness and avoid decreases thereof.

Drawing from functional perspectives, we will then - in the second part of this chapter - discuss theoretical and empirical studies supporting the idea that a taxonomy of general goals can be derived from the spectrum of human emotions. Subsequently, we will present two empirical studies of our own that provide further support for the association between emotions and goals, in the third part of this chapter.

\section{IDENTIFYING THE GENERAL GOALS OF BEHAVIOR}

Although various concepts have been proposed from which the general goals underlying behavior ostensibly derive, researchers have never reached agreement conceming the contents of a taxonomy of goals (for overviews, see: Austin \& Vancouver, 1996; Godsil, Tinsley, \& Fanselow, 2003). 


\section{Problems concerning previous conceptions of fundamental goals}

In the early twentieth century, instinct theorists (e.g., Lorenz, 1937; McDougall, 1908) tried to derive general goals directly from observed pattems of behavior. They proposed that observed action-patterns in animal and human behavior were caused by various instincts. As human behavior, however, proved almost infinitely diverse, and the instincts supposedly constituted hereditary mechanisms, the lists soon grew too large to provide a plausible account of general motives. Other researchers (eg., Murray, 1938) focused on psychological needs as the origin of goals. Needs are roughly defined as qualities of experience that humans require in order to thrive ( $\mathrm{cf}$. Sheldon, Elliot, Kim, \& Kasser, 2001). Rather than being genetically determined, like instincts, needs are also acquired through leaming and development. Once more, however, there was no apparent limitation to the number of needs that had to be invoked in order to account for behavioral diversity. Hence, these early approaches, although based on objective criteria (ie., observed behavior), suffered form a lack of parsimony.

More recent endeavors follow Maslow's (1943) suggestion that needs are hierarchically organized, with more specific needs presenting means to attain higher-order needs. Basically, they try to identify meta-categories of goals by having people compose and/or rate extensive lists of goal-constructs on various dimensions, like importance (Reiss \& Havercamp, 1998; Schwartz, 1992), amount of satisfaction derived from attainment (Sheldon et al., 2001), or perceived similarity (Chulef, Read, \& Walsh, 2001). For instance, lists of values have proliferated in recent years as taxonomies of goals (e.g., Schwartz, 1992). Specifically, Schwartz (1992) tried to identify general goals by having people from various cultures rate the importance of extensive lists of values, mainly derived from Rokeach' (1973) pioneering work in this area. Cluster analyses of these ratings allegedly resulted in a taxonomy of universal goals.

This approach has been criticized, however, for relying too heavily on subjective methods (i.e., self-reports), both for the identification of goals as well as for determining their involvement in the generation of behavior (see: Barsalou, 1985; as cited in: Chulef, et al, 2001; Murphy \& Medin, 1985). The subjective approach assumes that people are capable of identifying the goals underlying their behavior by introspection. Nevertheless, research has demonstrated that people are often incorrect when asked to indicate the causes of their conduct (Nisbett \& Wilson, 1977) Moreover, certain goals may be more likely to be mentioned by people to guide their actions than others. For instance, autonomy, competence and relatedness are among the most frequently mentioned strivings in self-generated lists of goals (Sheldon, et al, 2001). However, more obvious (e.g., safety), mundane (e.g., wealth), or socially undesirable (e.g. power) motives are apt to be overlooked or neglected in this approach. In other words, constructs that are currently used to characterize goals lack an objective criterion for identifying them as basic constituents of a taxonomy of general goals. Hence, these subjective approaches cannot guarantee the development of a comprehensive and universal goal-set. 
What seerns to be required is an objective criterion to (1) determine the presence of a goal-directed process underlying a behavioral response, and (2) to differentiate between the general goals. Compare, for example, the problem of differentiating between different species of animals or plants in biology. Early approaches, like Linnaeus' (1756) taxonomy, were based on observed morphological similanities among organisms, yet failed to provide a satisfactory classification as it remained unclear which part of an animal or plant should be compared. Therefore, more recent approaches relied on objectively defined criteria, such as the potential of providing fertile offspring or genetic overlap. Similarly, the development of a systematic classification of fundamental goals also requires an objective criterion for the identification of goals:

The main problem contributing to the lack of resolve on this matter is that the question of what determines origin or function of goals still remains unanswered (eg, Austin \& Vancouver, 1996; Chulef et al, 2001; Sheldon et al, 2001). In other words, without a theoretically derived notion of why these goals came to guide behavior in the first place, determining their content remains a futile enterprise. Similarly, without having a notion of the function of a car, of what it is designed to do, it would be impossible to decide it's basic elements, and one could easily conclude that, given that all cars have chairs or headlights, they are devices to relax in or to use to see in the dark.

\section{An Evolutionary Approach towards the Identification of General Goals}

Pertaining to this issue, we subscribe to the position of researchers in the field of evolutionary psychology, who argue that fundamental goals originate form evolutionary requirements (e.g., Buss, 1999; Edwards, 1999; Tooby \& Cosmides, 1990). Goals refer to the function of a particular act of behavior, and determining function ultimately requires one to adopt an evolutionary stance as: "Natural selection is the only known process capable of accounting for complex functional design in living things" (Dawkins, 1986, as cited in: Tooby \& Cosmides, 1990, p.383).

From an evolutionary perspective (see Box 3.1), behavior in general should be considered a faculty that enables an organism to maximize its inclusive fitness.

It thus follows that the fundamental goals underlying behavior derive from recurrent conditions that presented opportunities or obstacles to maximize inclusive fitness. These conditions are commonly referred to as "adaptive problems" (cf. Tooby \& Cosmides, 1990). Hence, adaptive problems encountered consistently for long periods during the evolutionary development of a species, such as mate promiscuity, escape from predators, or the maintenance of one's position in a social group, spurred the development of psychological mechanisms generating a behavioral response aimed to overcome the problem or to seize the presented opportunity. For instance, feelings of jealousy usually follow signs of imminent adultery, like one's partner showing a lot of interest in someone else. Jealousy may be a beneficial trait that evolved precisely because it prevents the negative consequences of promiscuity. This idea is supported by empinical studies that show sex differences in feelings of jealousy (Buss, 1999; Shackelford, 2000). Whereas men report feeling more jealous after 


\section{Box 3.1: "Evolution 101"}

Basically, evolution by natural selection holds that characteristics or traits develop because they enable an individual to produce more offspring (Darwin, 1859). The number of an individual's progeny in subsequent generations is also called an individual's fitness. A trait that contributes to an individual's fitness is called an adaptation or an adaptive trait. As individuals vary in the extent to which they possess or exhibit a trait, some will produce more offspning than others. Consequently, the frequency of individuals showing this trait will increase over the proportion that does not or does, but to a lesser extent This process is called natural selection. The requirement, of course, is that the trait is hereditary (i.e., can be transfenred to future generations). The total number of heritable traits is called an individual's genotype. As individual's not only share their genotype with their own offspring, but also with the offspring of their relatives, we talk about inclusive fitness, meaning the total amount of copies of oneself in the next generation

Consider for example the pepper coloured moth (Catocala relicta). Because of his peppery colour, the moth was well camouflaged against predators sitting at the trunk of birchtrees, his natural habitat. Nevertheless, with the rise of the industrial revolution in the 18th century, the bark of the birch-tree darkened due to the soot in the air, and the moth became more visible to predators. Nevertheless, moths that were slightly darker were also slightly less visible and hence more likely to escape from predation. Within a few generations, pepper-coloured moths turned into substantially-less-pepper-coloured moths: An excellent example of natural selection at work.

Underlying evolutionary psychology is the idea that the mechanisms underlying our behavior constitute adaptations as well. Naturally, camouflage is not the only means to escape from predators, for example. Behavioral strategies may serve equally well, hence it seems likely that natural selection also shaped the mechanisms underlying our behavior.

their (female) partner commits an act of sexual infidelity, females, on their other hand, report more jealousy if their partner commits "emotional infidelity", for instance by an intimate friendship with another woman. This pattern in emotional responding to infidelity follows logically from predictions derived from evolutionary theory. As the greatest cost to female fitness would be to raise children without the help of her partner, emotional ties of that partner with another female constitutes a substantial threat On the other hand, male fitness would be more strongly impaired by raising offspring that was not his own. Hence, female promiscuity constitutes a greater threat to men. Apart from these differences in pattems of elicitation, the potential adaptive value of jealousy also shows from the behavior it usually instigates. For instance, getting angry with a potential nival or making increased efforts to 
please one's partner to prevent infidelity, and often, breaking-up of a relation after it has happened (Buss, 1999).

The previous example was not randomly chosen. In the following paragraph we will argue that emotions constitute exactly the psychological mechanism by which behavior is motivated with respect to the demands imposed by the present situation. Before we continue to outline this proposal, let us first address some comments to an evolutionary approach to identifying fundamental goals.

\section{Some comments on the evolutionary approach}

Not everyone shares the view that fundamental goals can be derived from adaptive problems (see: Rose \& Rose, 2000, for an overview). Critics usually ascribe to either or both of the following (two) objections:

Some argue that an evolutionary approach to the origin of fundamental goals would imply that human behavior is motivated by a single goal, that is, the strive to maximize fitness. Nevertheless, this does not seem to be the case, as we do not witness daily queues of adult men in front of the sperm banks, which from an evolutionary viewpoint, would be an extremely beneficial strategy to increase one's fitness. What seems to be overlooked by these skeptics, is that fitness is a long-term future consequence of present acts, and not a goal that can be directly observed in a way that it can be used to regulate behavior (cf. Tooby \& Cosmides, 1990). Therefore, rather than resulting in a general motivation to enhance fitness, evolution has produced (conscious) strivings towards various intermediate goals that in general appeared probabilistically associated with the enhancement of fitness. In other words: "humans are adaptation executers, not fitness-strivers" (Tooby \& Cosmides, 1990, p. 420).

The second objection to adaptive problems as the origin of fundamental goals is that evolutionary reasoning entails post-hoc argumentation that cannot be confirmed nor falsified (e.g., Buller, 2002; Buller \& Hardcastle, 2000). Specifically, it is always possible to ascribe adaptive functionality to an observed psychological faculty by invoking a set of conditions in an ancestral environment that spurred the evolution of an adaptive mechanism underlying it. In other words, this practice "postulate[s] mechanism isomorphous with outcomes" (Hinde, 1995, p. 51). However, as Gould (2002, p. 9) also questions: "How can we possibly know in detail what small bands of hunter-gatherers did in Africa two million years ago?". Such information would indeed be required to show the validity of adaptive interpretations of present-day psychological mechanisms. We do not contest this argument and agree that it is ultimately impossible to know for certain what particular adaptive problems guided the evolution of motivational systems underlying behavior. Although common sense reasoning might lead to some tentative candidates, this cannot be equated with scientific proof.

However, it should be noted that we only claim that adaptive problems constitute the origin of fundamental goals, without making any assumptions about the nature of these problems. The contribution of an evolutionary stance is not to prove adaptive tales but to 
generate tentative hypotheses conceming the function of (psychological) traits, like emotions. Furthermore, the view of adaptive problems as origins of fundamental goals, which in itself cannot be refuted, excludes certain suggestions concerning to the type and content of fundamental goals. For instance, the goal to become wealthy could never be a fundamental motive of human behavior as money was certainly not a commodity present in ancestral environments. Hence, making a lot of money, though guiding behavior at a conscious level, should nevertheless be considered a means to achieve an underlying goal. This example illustrates how adaptive considerations serve as an heuristic to identifying the likely candidates of fundamental motives by imposing a restriction on the range of goals that can be presumed fundamental and as such presents a basis for determining the vallidity of any proposed taxonomy.

\section{EMOTIONS AS THE FUNDAMENTAL GOALS OF BEHAVIOR}

In the present paragraph we will introduce our argumentation for emotions as the general goals underlying human behavior. We will develop our view of emotions as the fundamental motivational systems of human behavior by taking a functional approach to emotions (e.g. Keltner \& Gross, 1999).

\section{The functional approach to emotions}

Ever since William James' essay entitled: "What is an emotion?" (1884), researchers have been occupied by identifying the nature of an emotional episode. Answers to this question have been framed at all levels of psychological analysis, ranging from the neurological (e.g. Buck, 1999; Damasio, 2000; LeDoux, 1996; Panksepp, 2000), the physiological (Cacioppo, Berntson, Larsen, Poehlmann, \& Ito, 2000), cognitive (e.g., Lazarus, 1991; Scherer, 1999; Smith \& Ellsworth, 1985), to the behavioral - expressive (eg., Ekman, 1993; Frijda, 1986). This has accumulated in a vast anay of emotion definitions (Kleinginna \& Kleinginna, 1981). Although each of these views may appear equally valid (Frijda, 2000), attempts to phrase a complete definition of "an" emotion would certainly create controversy.

Therefore, rather than to detemine what constitutes the essential psychological or biological nature of an emotion functional approaches focus on the question why humans exhibit the host of physiological, cognitive and behavioral responses that have been identified as characteristic constituents of an emotion. This shift in perspective has evened the ground for a new conceptualization of emotions, and within the functional perspective, emotions are commonly described as: acute, episodic, biologically based, patterns of perception, experience, physiology, action, and commumication that occur in response to specific challenges and opportunities of goal-relevance (cf: Keltner \& Gross, 1999). The functional perspective can be summarized by the following propositions:

1 The emotions system constitutes the primary motivational system underlying goaldirected behavior in humans (e.g., lizard \& Ackerman, 2000). 
2. The mechanism underlying an emotional episode evolved in response to adaptive problems, which thus define the fundamental goals of behavior (e.g, Cosmides \& Tooby, 2000; Plutchik, 1991).

3. Discrete emotions serve distinct functions in goal striving that cannot be reduced to the overall valence of the emotion (e.g, Buck, 1999; Ekman, 1992; Frijda, 2000; Roseman, Wiest, \& Swartz, 1994).

4. In order to produce goal-directed responses, emotions orchestrate ongoing cognitive and physiological processes underlying the generation of behavior (e.g. Oatley \& Jenkins, 1996).

In the following, we will provide an extensive discussion in support of the basic tenets of the functional perspective. Specifically, we will present evidence for the necessary involvement of affective experiences in goal-directed action, supporting the view of emotions as the primary motivational system. Subsequently, we will outline several characteristics of emotional processes indicating discrete emotions to be associated with distinct goals in terms of elicitation, motivational consequences, and in their influence on cognitive processes. Finally, we will discuss research indicating the possibility to objectively identify the occurrence of an emotion and to differentiate between specific emotions.

Readers who are familiar with functional research on emotions or willing to accept the basic tenets as listed above can also skip to the summary on Page 69, and continue with the empirical part of this chapter.

\section{Emotions as fundamental motivational systems}

The essential involvement of emotional processes in goal-directed behavior is perhaps most compelingly demonstrated in the work of Antonio Damasio and his co-workers. In his book, Descartes Error, Damasio (1994) describes his research with patients who have suffered damage to their frontal lobes. As a result, these patients have become "emotionally flat". While retaining their cognitive abilities, these people appear to have lost their decisionmaking abilities, pondering endlessly over seemingly insignificant decisions whilst neglecting other, more important ones. In a series of experiments (Bechara, Damasio, Tranel, \& Damasio, 1997), they had these patients engaging in a gambling task, selecting cards from either of two decks. Choices from Deck $A$ ultimately yielded in a net gain, whereas choices from Deck B ultimately resulted in loss, although participants were unable to calculate with precision the payoff of each deck. Galvanic Skin Responses (GSR) were measured before the selection of cards. Healthy participants, even before explicitly being aware of the payoff from each deck, started to show GSR associated with negative anticipatory affect after about ten rounds of choice when selecting cards from Deck $A$ and started to restrict their choices to Deck B. Frontal lobe damaged patients never showed such anticipatory GSR and kept selecting cards from the risky deck, even after they realized this resulted in loss. The researchers concluded this occurred because of their emotional impaiment; these patients were 
insensitive to future consequences of their actions, demonstrating the necessary impact of affective expenience for goal-directed behavior.

The function of emotional processes in goal-directed behavior is substantiated in many lines of research (see: Austin \& Vancouver, 1996, for an overview). To clarify this function, goal-directed behavior is often compared to a feedback-control system, like a themostat, describing a cycle of goal-setting, goal-striving, and -monitoring (Gollwitzer \& Moskowitz, 1996). Emotional responses have been argued to indicate successes or failures in the attainment of goals (Higgins, 1997), or to signal the rate of goal progress (Carver \& Scheier, 1998).

From a functional perspective, emotions are regarded as specifying behavioral goals themselves, in accordance with the specific demands imposed by the present situation (Izard \& Ackerman, 2000; Keltner \& Gross, 1999). Darwin (1872) was the first to suggest that emotions evolved for their contribution to individual survival. Nowadays, many researchers share the view that emotions constitute mechanisms that prionitize goals for individual behavior in a way that during the course of evolution has proven most successful in enhancing the inclusive fitness of individuals (e.g., Plutchik, 1991; Tooby \& Cosmides, 1990). Consider reciprocal altruism: the cooperation between unrelated members of a social group (Trivers, 1971). The opportunity for reciprocal altruism presents an adaptive problem to animals living in social groups. Helping individuals to whom one is not related is beneficial (in an evolutionary sense) only if others will reciprocate the favor. Whereas mutual cooperation would likely increase the fitness of both partners, it is also nisky if an individual receives help but will not retum it in future interactions. Several emotions are assumed to have developed in response to these problems associated with reciprocal altruism. For instance, feelings of anger (Berkowitz, 1989) and suspicion will prevent victimization to cheaters. Feelings of gratitude, on the other hand, often follow instances of charity and appear related to increased tendencies towards cooperation in the future (McCullough, Kilpatrick, Emmons, \& Larson, 2001). Additionally, feelings of guilt are presumed to inhibit cheating or to induce compensatory action after failures to reciprocate (Lewis, 2000).

From a functional perspective, specific emotions signal particular problems requiring different solutions. Thus emotions motivate behavior that deals with these problems. This view primarily underlies our proposal to apply the spectrum of emotions as the basis for a taxonomy of fundamental goals. We will further elaborate on the signaling and motivational function of discrete emotions in the subsequent section.

\section{Characteristics of Emotional Processes}

As stated, distinct emotions (e.g. interest) supposedly signal the implications of the present situation (reading this dissertation) to maintain or realize a particular goal (acquiring novel, useful information). To accomplish this goal, psychological processes (e.g., focusing attention, activating relevant knowledge from memory) are recruited and action (continue reading) is motivated to achieve this goal. In the following the goal-related nature of emotions will be 
outlined by discussing the activation properties of discrete emotions, their motivational capacities, and their influence on cognitive processes.

\section{Emotion Elicitation}

According to both classic appraisal theories, as well as recent functional perspectives, emotions are considered to anise in response to cues signaling the relevance of the current circumstances for important personal goals. Appraisal accounts of emotion elicitation emphasize the cognitive process by which situations or events are analyzed along a number of dimensions that ultimate determine its relevance for individual concems (e.g., Lazarus, 1991; Oatley \& Jenkins, 1996; Scherer, 1999). Although people may differ in their appraisal of a specific event, similar appraisals always accompany the elicitation of the same emotion. Knowledge of the content of these appraisals; therefore, helps us understand the antecedent conditions of specific emotions. Smith and Ellsworth (1985) identified six basic dimensions: pleasantress, certainty, attention, control, anticipated effort, and responsibility on which various emotions can be differentiated Supposedly, environmental stimuli vary with respect to these dimensions, which causes them to elicit different emotions. For example, anger will be aroused if an event is appraised as unpleasant, one is certain about what happened, and perceives the event as caused by someone else (other-responsibility). (Note that not all dimensions need to be involved in the elicitation of a particular emotion) Research indicating that various emotions can indeed be demarcated with respect to these appraisal dimensions, shows that different perceptions of goal-relevance indeed cause different emotional responses (Smith \& Ellsworth, 1985). Furthermore, experimental manipulation of appraisals on a particular dimension (responsibility) by having participants attribute events either to oneself or another person caused different emotional responses to an ambiguously described event (Neumann, 2000). Whereas repeated attribution to oneself elicited guilt (characterized by self-responsibility), extemal attributions resulted in angry responses (characterized by other responsibility). The appraisal approach nicely captures the informational value of emotions as signals of goal-concem.

Nevertheless, the appraisal view has been criticized for putting too much emphasis on cognitive processing to mediate the elicitation of emotions (e.g. Izard, 1993; Zajonc, 1998). Zajonc's studies on mere exposure (1968) and affective priming (1980), have compellingly shown the relative independence of affect arousal from mediation by cognitive processes. For instance, in a series of experiments, subjects were asked to evaluate neutral symbols (Chinese characters) that appeared on a computer screen (Murphy \& Zajonc, 1993). The characters were preceded by neutral, positive, or negative stimuli (smiling or frowning faces). The faces were presented for durations too short to be consciously perceived. Nevertheless, results indicated that Chinese characters that were preceded by positive stimuli were evaluated more positively than characters that were preceded by a frowning face. This shows that basic affective responses can be imvoked virtually without any cognitive processing.

The functional perspective places fewer demands on the amount of cognitive processing involved in emotion induction, and thus provides more hospitable grounds to 
evidence of automatic activation. From a functional view; emotion-eliciting cues are specfifed in relation to the particular adaptive problem the emotion system was designed" to solve. These cues, therefore, are presumed to relate to situational characteristics that (duning the course of evolution) have proven constant and reliable indicators of conditions (adaptive problems) that favor a particular response over another in order to optimize inclusive fitness (Tooby \& Cosmides, 1990). For instance, low blood sugar signals a depleted nutritional state, seeing your partner showing interest in another individual may imply imminent promiscuity, a fast approaching large object may cause physical harm. According to the functional perspective, emotions are based on innate biological systems, associated with specific triggers. The ethological literature (eg., Lorenz, 1937) identifies various species-specific behavioral patterns to be automatically activated by particular cues, also referred to as "signstimuli". Such views imply bottom-up analysis of stimuli, whereas the appraisal perspective is better characterized as a top-down process (Buck, 1999).

This need not imply, however, that appraisal and top-down views are incompatible conceming their account of emotion elicitation. Recent neurological data has shown the existence of different pathways along which emotion-eliciting cues are processed in the brain ('LeDoux, 1996). Investigating responses to fearful stimuli, LeDoux identified a "low road", leading from the sensory thalamus to the amygdala, and a "high road", which leads from the thalamus via the sensory cortex to the amygdala. The low road enables a subconscious, quick-and-dirty processing of incoming information allowing a quick reaction in response to potentially harmful situations. The high road, on the other hand, creates a detailed analysis of the stimulus and may modulate the initiated response. The existence of two pathways for processing of emotional (in this case, fear-related) stimuli, integrates notions of emotion elicitation as provided by appraisal and top-down accounts. Indeed, most researchers nowadays assume the coexistence of automatic and extended appraisal processes in the elicitation of emotions (e.g., Buck, 1999; Ekman, 1992; Zajonc, 1998). Obviously, most situations of relevance to social psychologists will require more elaborate appraisals before they acquire meaning as antecedents of emotions (Johnson-Laird \& Oatley, 2000).

\section{Motivational properties of emotions}

Goal relatedness is not only apparent from the elicitation of particular emotions, but also from the behavioral response that follows an emotional experience. For instance, fear not only signals danger, but also motivates escape.

Several authors have indeed attributed a central place to the behavioral consequences as defining characteristics of emotions (e.g., McDougall, 1908; Tomkins, 1962; Frijda, 1986). These perspectives focus on the desire or impulse to act (i.e., the motivation) associated with an activated emotional state. Different views have emanated from considerations of the relation between emotion and motivation (see Frijda, 2000, for an overview). Some conceive emotions, such as fear or lust, as causes of motivations like the desire for safety or to possess (e.g., Johnson-Laird \& Oatley, 1992). Others regard emotions as the consequence of activated 
dispositions for actions. For instance, McDougall (1908) refers to emotions as the actualizations of instincts. Similarly, Buck (1999) speaks of emotions as the readouts of motivation. Some (e.g. Bindra, 1959) have argued to abandon the distinction altogether. These views, however, share the notion that behavior is causally linked to the activation of emotional states (Frijda, 2000).

In support of the idea that emotions are associated with distinct behavioral goals, Roseman and colleagues demonstrated that specific emotions are accompanied by the experience of distinct action goals, or "emotivations", defined as the end-result towards which behavior is directed (Roseman et al, 1994). (Additional support for the association of emotions with specific goals will be presented in Study 3.1 at the end of this chapter.)

\section{Table 3.1. Functional Description of Discrete Emotions}

Emotion

Behavioral Goal / Function

Anger Overcome an obstacle. / Persistence in goal-striving (Berkowitz, 1989).

Fear / Distress Avoid physical or psychological harm. / Maintain personal safety.

Disgust Reject source of physical (i.e. toxic) or psychological (i.e. unfamiliar ideas or habits) contamination. / Maintain health (Rozin, Haidt \& Mccauley, 2000).

Interest Explore. / Learning of skills and competences.

Excitement Continue engagement in an act. / Learning of skills and competences.

Affection / Seek proximity to other individuals. / Formation of social bonds

Love (Eisenberg, 2000).

Concern / Pity Help others: / Maintenance of collaborative bonds.

Guilt Induces reparative action. Anticipation of guilt prompts one to forego immediate self-interest in favor of long-term benefits from cooperation (Frank, 2004). / Maintenance of collaborative bonds.

Awe / Respect Conform to others. / Preservation of social hierarchy.

Shyness Reservation in social interactions / Avoidance of social exclusion (Lewis, 2000).

Shame Anticipation of shame inhibits transgression of social norms. I Avoidance of social exclusion (Lewis, 2000).

Pride Anticipation of pride instigates achievement according to personal and social standards. / Achievernent of social status (Lewis, 2000).

Contempt Ignore other people. / Enables the pursuit of self-interest at the expense of others. 
Table 3.1 presents a description of 15 emotions and associated behavioral goals. Furthermore, for each emotion, the table tentatively describes how its associated goal contributes to the solution of some adaptive problem, which is considered to be the function of the particular emotion. These 15 emotions are selected from the influential catalogues of human emotions of Ekman (e.g., Ekman, 1992) and Izard (e.g. Izard, Libero, Putnam, \& Haynes, 1993). Notably, the emotions of happiness and sadness, which are also listed in these catalogues, have been excluded from our list. The rationale behind this was that they predict general goal achievement and loss respectively, and therefore are less indicative for the involvement of more specific behavioral goals. However, we added the emotions of pride and affection/love, as these emotions, though not mentioned by Izard and Ekman, have been associated with behavioral goals and/or adaptive functions by others. (Additional literature references pertaining to specific emotions are explicitly mentioned in the table).

Recent studies on the behavioral consequences of regret and disappointment nicely illustrate how even quite similar emotions, nevertheless instigate different actions (Zeelenberg \& Pieters, 2004). These studies examined the behavioral tendencies of customers who were dissatisfied with a service. Results indicated different behavioral consequences of both emotions. Whereas feelings of disappointment did not result in overt action but only in talking to others about the bad experience, regret instigated switching to a different service provider. These responses were consistent with the appraisal patterns associated with the emotions. Specifically, both are associated with outcomes that have not met expectations, yet in case of regret (but not disappointment), people also have the feeling that they could have prevented the outcome (Zeelenberg, van Dijk, Manstead, \& van der Pligt, 2000). Hence, regret will instigate a different course of action in future decisions, but disappointment will not. (In Study 3.2, we present additional support for behavioral differences related to distinct emotional states.)

Of course there is no necessary relation between an emotion (e.g., guilt) and a specific action (e.g., buying flowers) undertaken to accomplish the general goal associated with the emotion. Depending on the situation, a general goal (i.e, to compensate for transgressing a standard of conduct) may be achieved in many possible ways. This is referred to as the "equifinality principle" of goal-activation (eg., Kruglanski, 1996). Due to the equifinality principle, general goal-constructs like emotions (e.g. guilt) are unlikely to be strongly correlated to specific actions, as there are many ways to attain a goal (e.g., buying flowers, cooking dinner, doing the laundry). Goals, however, do provide insight into the common determinants underlying these various actions. Relative to general goals, EV models are very good at predicting the occurrence of specific acts, yet fail to provide an encompassing view of behavioral patterns. As will be argued in the following chapters, integrating motivational and reasoned-action perspectives will thus combine the merits of both approaches.

Emotional influences on behavior can be mapped along a voluntary - involuntary dimension. Especially Frijda (1986) has stressed that emotions often instigate an involuntary, yet non-habitual form of action control In a particular situation, the goals for action are thus 
prioritized by the emotional state. This is what Frijda (e.g., 1986) refers to as "control precedence" of an emotion induced goal, which means that it may overnule other goals in the control of behavior. In this sense, an emotional expenience overrules ongoing behavior in an impulsive or reactive manner. One of the earliest descriptions of this feature of emotional influence on behavior was given by Darwin (1872, p. 38), in describing the reflexive nature of emotional responses:

I put my face close to the thick glass-plate in front of a puff-adder in the Zoological Gardens, with the firm determination of not starting back if the snake struck at me; but as soon as the blow was struck, my resolution went for nothing, and I jumped a yard or two backwards with astonishing rapidity. My will and reason were powerless against the imagination of a danger, which had never been experienced.

This example nicely illustrates the many instances in daily life in which we are simply struck by an emotional response compelling us to actions, though sometimes unnecessary or even detrimental, which we nevertheless find hard if not impossible to control: A startle when perceiving the neighbors' dog approaching (though safely chained), promiscuous excesses after meeting this really incredible person at a party, caving to the desire for a cigarette, a burst of pity for a little child that has fallen fromit's bike.

Given the motivational capacities of emotions, it follows that individual differences in goal-directed behavior are ultimately the result of individual differences in emotional responses to situational stimuli (e.g., Izard \& Ackerman, 2000; Lemenise \& Arsenio, 2000). Support for individual differences in emotionality comes from research on infant temperament, indicating stable individual differences in onset, duration, and expression of affective reactions, emerging in early childhood (Lemerise \& Arsenio, 2000). Furthermore, twin-studies have demonstrated heritable similarities in emotional responding. Finally, studies have recorded stable individual differences in the extent to which (11 discrete) emotions were experienced in daily life for a period of up to 3 years (Izard et al, 1993).

Furthermore, authors adhering to the temperamental view of personality, state that certain personality traits represent endogenous differences in activation thresholds for emotional stimuli (Abe \& Izard, 1999; Elliot \& Thrash, 2002; lzard et al, 1993). Results indicating that neurotic individuals were more prone (compared to extraverts) to show negative emotional response following a negative affect induction task. whereas the opposite was found on measures of positive emotions following a manipulation of positive affect, suggests that personality types are indeed associated with different thresholds for emotion activation (Larsen \& Ketelaar; 1991).

\section{Emotional Influences on Cognition}

In order to generate adaptive behavioral responses, emotions can be expected to tune the various psychological processes involved in producing such a response. The general assumption underlying the functional approach holds that each discrete emotion serves a 
distinct function in the way it organizes the encoding retrieval and processing of information Indeed, a large body of empirical evidence shows that emotions can profoundly influence cognitive processes (for reviews, see: Forgas, 1995; Kruglanski, 1996; Oatley \& Jenkins, 1996; Schwarz \& Clore, 1996). We will briefly introduce these effects as they are relevant to the hypotheses conceming the effects of emotions on the behavioral influence of outcome expectancies as will be outlined in Chapter 4 .

Two mechanisms have been proposed to mediate the impact of emotion on cognitive processing, a direct mechanism and an indirect mechanism (cf. Schwarz; 2000). The direct mechanism is explicated in the Affect as Information Model (Schwarz \& Clore, 1996). According to the model, people sometimes use their emotional state or their emotional response to an object as a judgment heuristic. Rather than evaluating all the relevant aspects of an object or situration, beliefs, attitudes, judgments and choices are simply made by questioning oneself: "How do I feel about it?". Supportive of this model are the numerous instances of affective influences on judgment, showing that people are likely to evaluate a target more positively when in a happy than in a sad mood. For instance, when called on a bright sunny day, or after seeing a happy movie, people reported higher levels of lifesatisfaction, than when called on a grey rainy day.

Indirect influences of emotion on cognition occur as a consequence of affective priming processes, which constitute instances of emotion-congruent influences on cognitive processes (e.g., Forgas, 1995; Klauer, 1998). To describe this mechanism, Oatley and Jenkins (1996) conceive of an emotional state as a particular "mode of brain organization", in which the operation of vanious psychological processes is orchestrated in accordance with the demands imposed by the current situation. For instance, the retrieval of information from memory occurs in an emotion-congruent fashion. People in a happy mood, for example, appear to report more positive events or traits of a previously encountered object, whereas the opposite is true for sad people. From a functional perspective, emotion-dependent memory retrieval ensures the retrieval of information required for making appropriate decisions in a given situation (Cosmides \& Tooby, 1999).

Additionally, affective states have significant influence on the kind of information people will focus on. Emotion congruent details will be more readily perceived and tend to receive greater attention than emotion-incongruent information does. For instance, after inducing a happy mood state (with music), people were better at discriminating between non-words and words with positive connotation, whereas sad people performed better for words with a negative meaning (Innes-Ker \& Niedenthal, 2002).

Although most research investigating cognitive modulation by affective influences has taken a valence-based approach, discriminating only between positive and negative feeling states. Nevertheless, recent studies have also addressed the cognitive implications of inducing distinct positive and negative emotions (Lerner \& Keltner, 2000; 2001). Drawing on appraisal theory, the authors hypothesized that each emotion activates a cognitive predisposition to evaluate future events in line with the appraisal structure of that emotion, which is referred to as an "appraisal tendency". For instance, fear would be associated with 
the tendency to appraise future events as uncertain and externally controlled, whereas anger is associated with high certainty and a sense of personal control over a situation in order to test these predictions, the authors investigated the effects of anger and fear on risk perception. As expected, fearful people made more pessimistic estimates than angry people conceming the number of casualties caused by certain events (e.g, breast cancer, strokes, flood). Besides being more optimistic, angxy people were also more indined towards making risk-seeking choices (Lerner \& Keltner, 2000; 2001). These opposing patterns were found for both naturally occuming (ie, dispositional), as well as expenimentally induced states of fear and anger. Finally; risk estimates made in an angry emotional state matched those made in a happy state, which is also characterized by high certainty and personal control (Lemer \& Keltner, 2001). Quite similarly, it was found that angry people were more inclined to support punitive social policies; whereas empathy inductions resulted in a greater preference for reparative human service policies (Gault \& Sabini, 2000).

These findings convincingly demonstrate that specific emotions exert differential impacts on cognitive processes involved in judgment and choice, beyond positive and negative congruency effects.

\section{Identifying Emotions}

To conclude our discussion of emotions as general goals of behavior, we need to address how emotions meet the requirement of objective assessment that was imposed on a taxonomy of fundamental goals. Characteristically, emotional episodes are associated with bodily sensations; which is evident in everyday descriptions of particular emotional states. For instance, when in love, we feel butterflies in our stomach, and when angry, we are boiling inside. These bodily sensations are reflected in psychophysiological conrelates of an emotional state (e.g. Cacioppo, et al, 2000). Measures of autonomic- (e.g. galvanic skin responses, cardiovascular activity), and central nervous system activity (e.g, cortical brain activity as assessed by EEG scans), provide convergent evidence that the onset and intensity of an emotional experience can be objectively determined. Because they are "embodied" ( $c$. Lazarus, 1991), emotions exceed the level of cognitive representation at which other goalconstructs, like needs or values; are specified. The physical processes thus justify our proposal to use the spectrum of emotions as the primary index of general behavioral goals.

Nevertheless, psychophysiological measures differentiate poorly between specific emotional states (Cacioppo, et al, 2000; Larsen \& Fredrickson, 1999). Fierce debates still surround the questions of how to identify specific emotions (Stein \& Oatley, 1992). Two approaches towards determining specific emotional states have stood out in recent years. These are the identification of facial expression and the search for neural correlates of distinct emotional experiences.

Traditionally, researchers have classified emotions by universal facial expressions (e.g. Tomkins, 1962; see Ekman, 1993 for an overview). Evidence of universality in facial expression is consistent with Darwin's (1872) view that the emotions signaled by these facial expressions are the product of evolution and constituted adaptive means of communication 
between individuals, conveying information about what is likely to happen. Although facial electromyography recordings of the muscle contractions involved in these facial expressions may provide an objective means of identifying emotions, two objections have been raised against this way of developing emotion taxonomies. First, facial expressions likely evolved as communicative signals (Darwin, 1872). Nevertheless, as argued by Trivers (1971), from an evolutionary perspective, communicative signals need not necessarily convey truth, as it may be beneficial to keep others unaware of your intentions or feelings. Hence, it may - but not need - be beneficial from an evolutionary perspective for certain emotional states to have become related to clear emotional signals. For instance expressing anger may be a way of telling someone to back off without actually having to engage in physical combat. On the other hand, expressing guilt is unlikely to benefit an individual and is therefore unlikely to have become associated with a characteristic facial expression. Secondly, recent studies seem to open up the possibility that early research on facial expressions may have been overly restrictive, in that they only focused on prototypical expressions (Rozin \& Cohen, 2003). Other emotional expressions may be more variable, but nevertheless consistently associated with distinct emotions.

More recent approaches to identifying emotions rely on improved methods for identifying brain substrates of psychological faculties, such as MRI and other techniques for measuring activity in- and identifying neural anatomy of the brain. Separate neural circuits (e.g., LeDoux, 1996; Panksepp, 2000; Weddell, 1994), as well as various chemical substrates (e.g. Buck, 1999; Burbach, 2000) have been identified for most emotions that are generally conceived as fundamental (eg., hapiness, sadness, fear, anger, disgust, etc.), as well as some of the so-called moral emotions, like guilt (Greene, Sommerville, Nystrom, Darley, \& Cohen, 2001; Moll, Oliveira-Souza, Eslinger, Bramati, Mourao-Miranda, Andreiuolu, \& Pessoa, 2002). The identification of distinct neural systems provides a promising opportunity to objectively identify emotions. Nevertheless, any taxonomy at the neuropsychological level must remain open-ended until the knowledge in this domain is more complete (Panksepp, 2000).

\section{Emotions as Fundamental Goals: Summary and Conclusions}

We outlined that the general goals underlying human behavior should derive from evolutionary requirements, and suggested that the spectrum of emotions presents an adequate index thereof. Empirical research and theoretical arguments from functional accounts of emotions seem to support this proposal (Keltner \& Gross, 1999). Notably, emotions play an essential role in goal-directed behavior (Carver \& Scheier, 1998; Damasio, 1994). Furthemore, discrete emotions are indeed associated with distinct goals, both in terms of their elicitation by environmental cues signaling situational relevance for a particuiar goal (Smith \& Ellsworth, 1985), and in terms of motivational consequences for action (Frijda, 1986; Roseman, et al, 1994). Moreover, emotions orchestrate cognitive processes in a goal-directed fashion (Schwartz \& Clore, 1996; Lemer \& Keltner, 2001). Finally, physiological measures provide convergent evidence for the objective assessment of the occurrence of emotional episodes (Cacioppo, et al, 2000), and ongoing development of neuro-imaging techniques is likely to provide an objective means to differentiate between specific emotions (Panksepp, 
2000). Emotions thus seem to meet the qualifications imposed on a taxonorny of general goals:

In the remainder of this chapter, we present two empirical studies in which we elaborate further on our proposal of emotions as fundarnental goals of behavior. In the first study (3.1) we establish relations between emotions and values, another construct proposed to explicate fundamental goals. In Study 3.2, we conducted several experiments in order to investigate how situation-specific goals for action and general goals from emotional states operate in concert to determine behavioral choice.

\section{STUDY 3.1: RELATIONS BETWEEN EMOTIONS AND VALUES ${ }^{2}$}

\section{Introduction}

If indeed emotions are associated with general goals, we should be able to establish meaningful relations between emotions and other general goal-constructs in the extent to which people experience those goals to influence their behavior and lives in general. In order to test this assumption, we assessed relations between values and emotions. Such relations have rarely been addressed before, and only in a particular context, like study choice (Myyry \& Helkema, 2001), capital punishment (Haddock \& Zanna, 1998) or cultural differences (Mesquita, 2001). To our best knowledge, this study presents the first systematic comparison of these relations in a comprehensive range of values and emotions.

As stated, values can be regarded as motivational constructs that specify abstract goals guiding people's actions across contexts and time (Schwartz, 1992; 1994). The most popular catalogue of values is probably Schwartz' Value Survey (SVS), a comprehensive list of 10 value types differentiated by their motivational goals, which are ostensibly derived from basic requirements of human existence. These value types are described in Table 3.2. Research has shown these values to be universal across cultures and ratings of the importance of these values have been shown to be predictive of a variety of actions (Bardi \& Schwartz, 2003; Feather, Norman, \& Worsley, 1998) including health-related behavior (Kristiansen, 1985).

As both values and emotions are presumably related to universal goals that direct behavior, we expected that people's ratings of values and of emotional experiences would be meaningfully related in terms of the common goals implied. Relations should appear both in individual disposition ratings as well as in response to a particular context or behavior. For example, people who report that they frequently expenience fear in daily life should attach great importance to security values, as both the emotion of fear and the value of security share the common goal of realizing safety. Furthermore, if people's attitudes towards a particular behavior (e.g. condom use) are predicted by the extent to which the behavior is perceived as congruent with safety values, we would expect perceived value congruence to relate to a decrease in the amount of fear expected when performing that behavior. The latter relation should even be stronger, as situational perceptions are more readily accessible to people than identity-related beliefs (Robinson \& Clore, 2002). 
Table 3.2. Definitions of Types of Values in the Schwartz Value Survey

\begin{tabular}{|c|c|c|}
\hline Value Type & Scale $\alpha$ & Definition \\
\hline Power & .65 & $\begin{array}{l}\text { Social status and prestige, control or dominance over } \\
\text { people and resources }\end{array}$ \\
\hline Achievement & .68 & $\begin{array}{l}\text { Personal success through demonstrating competence } \\
\text { according to social standards }\end{array}$ \\
\hline Hedonism & .70 & $\begin{array}{l}\text { Pleasure and sensuous gratification for oneself (pleasure; } \\
\text { enjoying life) }\end{array}$ \\
\hline Stimulation & .81 & Excitement, novelty, and challenge in life \\
\hline $\begin{array}{l}\text { Self- } \\
\text { Direction }\end{array}$ & .64 & $\begin{array}{l}\text { Independent thought and action-choosing, creating, } \\
\text { exploring }\end{array}$ \\
\hline Universalism & .79 & $\begin{array}{l}\text { Understanding, appreciation, tolerance and protection of } \\
\text { the welfare of all people and of nature }\end{array}$ \\
\hline Benevolence & .74 & $\begin{array}{l}\text { Preservation and enhancement of the welfare of people } \\
\text { with whom one is in frequent personal contact }\end{array}$ \\
\hline Tradition & .56 & $\begin{array}{l}\text { Respect, commitment and acceptance of the customs and } \\
\text { ideas that traditional culture or religion provide the self }\end{array}$ \\
\hline Conformity & .67 & $\begin{array}{l}\text { Restraint of actions, inclinations, and impulses likely to } \\
\text { upset or harm others and violate social expectations or } \\
\text { norms }\end{array}$ \\
\hline Security & .68 & $\begin{array}{l}\text { Safety, harmony, and stability of society, of relationships, } \\
\text { and of self }\end{array}$ \\
\hline
\end{tabular}

Note. Adapted from "Values and behavior: Strength and structure of relations" by A. Bardi and S. H. Schwartz, 2003. Personality and Social Psychology Bulletim, 29, p. 1208.

It should be noted that in principle, reciprocal relations exist between values and emotions. Correlations may arise, for example, due to the fact that behavior directed by a particular class of values (e.g. achievement) is likely to result in situations in which people experience a particular emotion (e.g. pride). On the other hand, frequent experience of a particular emotion (like interest) will instigate behavior from which people, for instance through self-perception, deduce that certain values (like stimulation) are important guiding principles in their daily lives. It should also be noted that one-to-one relations between values and emotions are unlikely because (1) mutual associations exist within the two classes of concepts, and (2) the two classes specify goals at different hierarchical levels (Kruglanski, Shah, Fishbach, Friedman, Chun, \& Sleeth-Keppler, 2002). Particularly alleged basic emotions (like fear) specify goals at a more fundamental level and may thus be related to more than one value (e.g. security, conformity and tradition), all of which constitute means to achieve the general goal of safety. 
Totest our expectations, we related people's importance ratings for each of the 10 value constructs of the SVS to their estimates of how frequently they experienced each of the emotions in Table 3.1 in daily life. In addition, to establish context-dependent associations, we asked people to indicate the extent to which they considered a particular action to be congruent or in conflict with each value (cf. Rosenberg, 1956), as well as the extent to which they expected to experience each of the emotions in Table 3.1 when performing the action These assessments were made for four health-related behaviors. Subsequently, people's attitudes towards each behavior were assessed. Values that were found to be predictive of these attitudes were then tested for relations with expected emotional responses. We decided to use values as predictors of attitudes because values, as allready stated, specify goals at a more concrete and accessible level than emotions and therefore should be easier to report and, in terms of content, more distinctly related to attitudes.

\section{Method}

Participants \& Procedure. Participants were students enrolied in introductory courses of psychology and health sciences at the University of Maastricht, the Netherlands. During classes participants completed two questionnaires, delivery of which was separated by one week. The first questionnaire (completed by 487 students, 438 females and 49 males, mean age 21 yrs., $S D=4.96$ ), which was similar for all participants, measured emotional and value dispositions. The second questionnaire (completed by 257 students, 228 females and 29 males, mean age 21 yrs., $S D=5.01$ ) assessed participants' attitudes, emotional response to, and perceived value-congruence with one of four target behaviors; condom use $(N=64)$, eating genetically modified (GM) food ( $N=66$ ), organ donation $(N=66)$, and intervening with random violence $(N=61)$. Participants were randomly assigned to the target behaviors. Overall, completion of both questionnaires took about 1 hour. Participants received a monetary reward of $€ 7$.

Measures: Emotional Dispositions. The first questionnaire measured individual emotional dispositions for each of the 15 emotions in Table 3.1, by asking participants to estimate the frequency with which they tended to experience these emotions in daily life, on a scale ranging from 1 (never) to 5 (very often). (See: Izard, et al, (1993) for a similar procedure)

Value Dispositions. Participants also completed the Dutch translation of the Schwartz' Value Survey (Schwartz, 1994). The SVS asks participants to rate 56 items assessing the importance of a wide range of values as "guiding principles in your daily life", on a scale ranging from $O$ (unimportant) to 7 (extremely important), with the additional option to rate a value item as -1 (contrary to $m y$ values). The values were presented by catchwords (e.g. "respect for tradition"), followed by a brief specification (e.g, "preservation of time-honored customs"). Previous analyses had indicated that the various items of the SVS relate to one of 10 types of universal values that can be distinguished on the basis of their motivational goals. As alpha coefficients derived from the present data were sufficiently high (see Table 3.2), item scores for each higher order value type were averaged into single measures. 
Responses to Behavior. The second questionnaire measured participants' responses to performing a particular behavioral act, using the same emotion and value items as in the first questionnaire. For the emotion items, participants rated the extent to which they expected to feel a particular emotion while performing the behavior compared to not performing the behavior on a 7-point scale ranging from -3 (a lot less) to 3 (a lot more). For instance: "If I use condoms, I will feel more/less fear while having sex than when I do not use condoms".

The value item list contained all items of the SVS, but rather than rating their importance, participants were now asked to indicate to what extent they considered performing the behavior (in)congruent with a particular value. Again, we used a 7-point scale, ranging from -3 (completely contradictory) to 3 (completely in accordance). Both emotion and value item scales had neutral midpoints.

Three items assessed participants' attitudes (good/bad, positive/negative, and favorable /unfavorable) to performing the target behavior on a 9-point scale with neutral midpoints. As alpha coefficients for the three attitude items proved sufficiently high for all target behaviors (all above .80 ), item scores were averaged into one measure of attitude to performing the behavioral act in question.

\section{Results}

\section{Relations between Emotion-and Value - Dispositions}

Correlations between dispositional measures of values and emotions are presented in Table 3.3. The pattem of correlations is generally consistent with the assumption that associations would be found between values and emotions that express similar goals.

In line with this assumption our results show, for instance, that the more important people consider power and achievement values, which both refer to the pursuit of selfinterest and success, the more frequently they experience pride, signaling personal success, and anger ${ }_{r}$ associated with persistence in goal striving. Whereas achievement values are also associated with interest and excitement, emotions related to intrinsic motivation, power is related to feelings of contempt. Experiencing contempt may to a certain extent be necessary when aiming for personal interest at the expense of others, as is implied in power values. The values of hedonism, stimulation, and self-direction all emphasize the importance of your own, independent thought and action, seeking pleasure and novelty. These values share a relation with the experience of interest and excitement, feelings that stimulate exploration and ensure enjoyment while engaged in action. Hedonism and stimulation are in addition negatively related to feelings underlying avoidance motivation, like shyness and, in case of stimulation, also fear. Self-direction differs from these two values by a relation with pride, illustrating the self-relevance of this value type. Associations between the values of universalism and benevolence and emotions like respect for others and affection are consistent with the common appreciation of others and of social bonds underlying these values and emotions. Both universalism and benevolence also show a positive relation with excitement, which corresponds to the notion that positive affect promotes social bonding. Benevolence, however, differs from universalism by its relation with feelings of concern for other people, 
illustrating the nurturing and cooperative tendencies implied by benevolence values, whereas universalism involves the protection of society as a whole and hence is also related to fear. Finally, the values of tradition, conformity, and safety, all express restrictions to avoid harm to the self and to others, and to circumvent violations of social norms. Understandably, the importance of these values is associated with feelings of concerm and respect for others. A few specific relations qualify the differences in the goals implied by these values. For instance, conformity relates positively to affection, suggesting that positive feelings for others underlie compliance to social norms. As expected, feelings of fear are related to importance adhered to safety (avoiding harm) and tradition (avoiding change).

Table 3.3. Pearson Correlations between Vallue and Emotion Dispositions

\begin{tabular}{|c|c|c|c|c|c|c|c|c|c|c|}
\hline notions & 3 & I & 뭎 & $\underset{n}{E}$ & 号 & 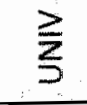 & 胥 & 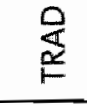 & 岂 & 㒴 \\
\hline Anger & $.15^{\prime \prime}$ & $.10^{\circ}$ & .06 & .02 & .03 & .01 & -.06 & -.02 & .01 & .09 \\
\hline Fear & -.01 & $.09^{\prime \prime}$ &. .06 & $-.10^{\circ}$ & .03 & $.11^{\circ}$ & .04 & $.11^{\circ}$ & .07 & $.12^{\prime \prime \prime}$ \\
\hline Disgust & .00 & -.02 & -.02 & .00 & .04 & .00 & $-.13^{\prime \prime}$ & -.03 & $-.14^{\prime \prime}$ & -.06 \\
\hline Interest & .01 & $.20^{\circ}$ & $.11^{\prime \prime}$ & $.21{ }^{*}$ & $30^{\prime \prime}$ & .03 & .09 & -.08 & .03 & .01 \\
\hline Excitement & .01 & $.11^{\circ}$ & $.13^{\prime \prime}$ & $.15^{\prime \prime}$ & $.13^{\prime \prime}$ & $.11^{\circ}$ & $.11^{\circ}$ & -.01 & .07 & .06 \\
\hline Affection & -.05 & .00 & .08 & -.04 & .01 & $.10^{\circ}$ & $.20^{*}$ & -.02 & $.10^{\circ}$ & $.09^{\prime \prime}$ \\
\hline Concern & -.03 & .01 & -.01 & -.06 & .01 & .07 & $.18^{\circ}$ & $.10^{\circ}$ & $.13^{*}$ & $.13^{\prime \prime}$ \\
\hline Respect & -.07 & .03 & -.01 & .01 & $.10^{\circ}$ & $.19^{* \prime}$ & $.17^{* \prime}$ & $.12^{\circ}$ & $.16^{\circ}$ & $.11^{\circ}$ \\
\hline Guilt & -.04 & -.01 & .01 & -.01 & -.02 & .06 & .08 & .00 & .00 & .01 \\
\hline Shyness. & -.06 & -.08 & $-.10^{*}$ & $-.12^{*}$ & .06 & .02 & .00 & .02 & $-.11^{\circ}$ & -.05 \\
\hline Shame & -01 & -.05 &. .03 & -.08 & -.04 & .05 & .04 & .05 & -.06 & .03 \\
\hline Pride & $.12^{\circ}$ & $.22^{\circ}$ & .06 & .08 & $.20^{\circ}$ & .00 & -.03 & $-.09^{\circ}$ & .03 & .03 \\
\hline Contempt & $.13^{\circ}$ & .07 & .08 & $.11^{*}$ & .06 & -.04 & $.14^{\prime \prime}$ & $-.10^{*}$ & $-.11^{*}$ & .02 \\
\hline
\end{tabular}

Note. $\mathrm{POW}=$ Power; $A C H=$ Achievement; $H E D=$ Hedonism; STIM $=$ Stimulation; $S-D I R=$ Self-Direction; UNI = Universalism; BEN $=$ Benevolence; TRAD = Tradition; CONF $=$ Conformity; SEC = security. $N=487$. $p<.05 . p<.01$.

These associations describe the majority of the correlations in Table 3.3. They could even be replenished by a few more, mainly negative ones, between values and emotions with conflicting goals, which we nevertheless considered less illustrative of the general idea that emotions and values share common goals. A few associations, however, are more difficult to explain, such as those between fear and achievement, contempt and stimulation, respect for others and self-direction, and a negative relation between shyness and conformity. 


\section{Relations between Emotions and Values in Response to Behavior}

We also investigated associations between values and emotions in relation to performing a particular behavior. Therefore; we first regressed attitudes towards performing the behavior onto ratings of congruence with each of the ten value types simultaneously. Then we assessed correlations between value types that significantly predicted people's attitudes and expected emotional responses to performing the act. These relations are summarized in Table 3.4.

Table 3.4. Pearson Correlations between Value Constructs Predictive of Attitudes towards Behavior and Expected Emotional Responses towards Behavior

\begin{tabular}{|c|c|c|c|c|c|c|c|}
\hline \multirow[b]{2}{*}{ Emotions } & \multirow{2}{*}{$\begin{array}{l}\text { Condom } \\
\frac{(N=64)}{\text { SEC }}\end{array}$} & \multicolumn{2}{|c|}{$\begin{array}{l}\text { GenMod } \\
(N=66)\end{array}$} & \multicolumn{3}{|c|}{$\begin{array}{l}\text { Organ Donation } \\
\qquad(N=66)\end{array}$} & \multirow{2}{*}{$\begin{array}{c}\text { Violence } \\
(N=61) \\
\text { CONF }\end{array}$} \\
\hline & & SEC & POW & UNIV & TRAD & 5-DIR & \\
\hline Anger & -.21 & $.29^{\circ}$ & -.22 & -.10 & -.11 & -.10 & .15 \\
\hline Fear & -.09 & $-.37^{\prime \prime}$ & .02 & -.16 & .18 & -.05 & .09 \\
\hline Disgust & -12 & -.16 & .07 & -.28 & -.05 & -.07 & $.43^{\prime \prime}$ \\
\hline Interest & -.12 & -.02 & .06 & .10 & -.01 & .22 & -.11 \\
\hline Excitement & -.24 & -10 & .10 & .11 & -.20 & $.29^{\circ}$ & .06 \\
\hline Affection & .20 & -01 & $-.24^{\circ}$ & .20 & .14 & .21 & $.26^{\circ}$ \\
\hline Concern & $-.26^{\circ}$ & -28 & -.01 & .22 & .22 & .15 & .08 \\
\hline Respect & .22 & .03 & -.12 & $.36^{\circ}$ & $.26^{\circ}$ & .02 & $.28^{\circ}$ \\
\hline Guilt & -26 & -.03 & .09 & $-.44^{\prime \prime}$ & .03 & .00 & .02 \\
\hline Shyness & -16 & .03 & -.02 & -.37 & -.16 & .02 & -.10 \\
\hline Shame & -16 & -15 & .19 & $-.26^{\circ}$ & -.01 & .07 & .08 \\
\hline Pride & .17 & .13 & -.16 & $.44^{\prime \prime}$ & .23 & $.35^{\prime \prime}$ & .00 \\
\hline Contempt & -29 & .03 & .20 & .00 & -.21 & $-.27^{\circ}$ & $31^{\circ}$ \\
\hline
\end{tabular}

Note. Condom = Condom use, GenMod = Eating genetically modified food; Violence $=$ Interfering when witnessing random violence; $S E C=$ Security; $P O W=$ Power; HJNIV $=$ Universalism; TRAD $=$ Tradition; $S-D I R=$ Self-Direction; CONF $=$ Conformity. "p<.05. p p o .01.

Attitudes towards using condoms were only predicted by security values $(\beta=.39, p=.07)$, suggesting that people experienced more positive attitudes towards condom use, as they perceived this behavior in to be in accordance with secunity values. Perceived congnience with security values, in tum, related to decreased expected feelings of guilt and concem for others but not with fear, suggesting that concem for others, rather than the self, underlies 
attitudes towards condom use. The negative correlation with contempt for others further stresses the other-orientation underlying perceived congruence of condom use with secunity values. Security $(\beta=68, p=.01)$ and power $(\beta=-39, p=.08)$ values predicted attitudes towards GM food The more people considered safety goals to be violated by eating $G M$ food, the more they expected to feel afraid and concem for others (see Table 3.4). The relation between security and anger may indicate the importance of frustrated safety goals in determining attitudes towards eating GM food. Power values did not show significant correlations with expected emotions. Attitudes towards organ donation were positively associated with universalism $(\beta=47, p, 002)$ and self-direction $(\beta=.25, p=.06)$ values and negatively with tradition values $(\beta=-32, p=.01)$. Also in response to behavior, tradition values were correlated with feelings of respect for others, mirroring relations among dispositional measures. Perceived congruence with universalism was again related to feelings of respect, but also to pride and a decrease of guilt, disgust, and shyness. Self-direction values were again found to correlate with feelings of pride, excitement, and surprisingly, a decrease in contempt. Finally, prediction of attitudes towards intervening with random violence by perceived congruence with conformity values $(\beta=.29, p=.08)$ might suggest that social desirability partly underlies these attitudes. This is illustrated by correlations of conformity with expectations of feeling disgust and contempt, associated with rejection of (socially) undesirable things. However, perceived congruence with conformity was also related to feelings of affection and respect for others.

\section{Discussion}

The results generally confirm our expectancies that (1) relations would be found between values and emotions that express similar goals and (2) that such relations would be stronger if values and emotions are assessed as responses to behavior rather than as dispositions. These associations indicate that the more people strive towards a certain goal, the more they experience the particular emotion that, according to functional accounts, constitutes the primary motivational force underlying behavior to attain that goal.

Although correlations were modest, we would like to emphasize that the pattern of relations rather than their magnitude was the primary test of our assumptions (cf. Bardi \& Schwartz 2003). We did not expect people's ratings of values and emotions to be strongly correlated, as the two show little semantic overlap, are measured on different scales, and relate to different psychological processes. Furthermore, whereas other studies investigating relations between values and emotions reported substantially (.55 in the study of Haddock \& Zanna, 1998) or slightly (.19 in the study of Myyry \& Helkema, 2001) higher average correlations, it should be noted that these studies used composite scales to measure overall affective response and emotional empathy, respectively, which increases reliability and hence correlation sizes. Furthemore, Haddock and Zanna (1998) asked people to list the values they personally thought were associated with capital punishment and subsequently calculated an overall value score. Such enhanced personal relevance of the cutcome measure will also boost correlations. Considering that we used single-item emotion measures and differently phrased scale measures, we regard the size of the observed correlations as satisfactory. 
Although virtually all of the observed relations could be readily interpreted in tems of the common goals of values and emotions, a few correlations were harder to understand. These anomalies, however, constituted only a very small (four out of 130 ) part of the total matrix. Also, some deviations were found in the pattems of correlations based on dispositional measures and those derived from responses to behavior. Discrepancies may have arisen because values and emotions relate to different psychological processes and hence may be differentially related to behavior. For example, condon use may be perceived as congruent with safety values due to descriptive knowledge of condoms as a means to avoid diseases. Yet at the expenential level of emotional responses, fear may not be experienced when having sex, either with or without condoms, unlike other feelings, such as guilt or concem for another person still, all relations found between measures of emotions and values in response to behavior can be understood regarding the underlying goals.

Evidently, the present study has its limitations. First of all, one could question whether emotions can be measured by means of self-reports. Indeed, both self-related and response measures of emotions tap semantic knowledge rather than emotional experience per se (Robinson \& Clore, 2002). Still, this semantic knowledge is ultimately based on direct experience with particular emotional episodes and may serve as a reliable proxy for the concept under investigation. Arguably, self-reports are the most common and perhaps even the best technique for measuring emotions (Larsen \& Fredrickson, 1999), especially when researchers acknowledge the various psychological processes tapped by different kinds of emotional self-reports (Robinson \& Clore, 2002). Nevertheless, studies relating on-line reports of emotional experience to pre-assessed measures of value importance would be a valuable line of inquiry for the future. Secondly, the generality of our results may be confined by characteristics of our research population. Nevertheless, the general goals underlying values and emotions are presumed to be universal for the human species. Therefore, relations between constructs measuring these goals inrespective of a particular context should transcend individuals, situations, and cultures.

The results of the present studies are in line with our conception of emotions as fundamental goals of human behavior. Furthermore, they illustrate the potential contribution from integrating general goal constructs in EV models to further our understanding of the factors that guide peoples actions in general, beyond perceived pro's and con"s that specifically relate to only one type of behavior.

\section{STUDY 3.2: INTERACTIONS BETWEEN EMOTIONS AND CHRONIC GOAIS ${ }^{3}$}

\section{Introduction}

In this study we extended previous research on the functions of emotions by demonstrating that the behavioral consequences of an (experimentally induced) emotional state may differ between individuals. We argue that these differences are related to variation in the extent to which individuals chronically strive for situation-relevant goals. In the following we will elucidate this argument by elaborating on the relation between emotions and goals: 


\section{Emotions and goals}

As stated, functional accounts emphasize the goal-related nature of emotions. They specify the ways in which emotions orchestrate psychological processes in order to generate an adaptive behavioral response, given the specific concems imposed by the present situation. conroborating these premises, research findings indicate that emotions can indeed be discemed by (a) the perceived charactenstics of the situation in which they occur (eg., Smith \& Ellsworth, 1985), (b) the action tendency that ensues from an emotional state (e.g. Frijda, 1986), and (c) by distinct categories of associated goals (e.g., Roseman, Wiest \& Swartz, 1994). Following the taxonomy of emotion-specific goals summarized in Table 3.1, we consider an emotion as a state of temporanily increased activation of a general goal.

A goal can be defined as a subjectively valued state-of-affairs or end-state of interactions between people and their (social) environment (Austin \& Vancouver, 1996). Irrespective of the way in which goals are conceptualized (e.g, as values, needs, life-goals, etc), many studies have indicated that behavioral differences are related to the extent to which people chronically (eg. Bardi \& Schwartz, 2003) or temporarily (Bargh \& Chartrand, 1999) strive for certain goals. Furthernore, results from priming studies suggest that both chronic (i.e., dispositionall) and temporal activation of a particular construct (e.g, a personality trait) affects psychological processes (like person perception) through the same underlying process; the level of construct accessibility (Bargh, Lombardi \& Higgins, 1988). Likewise, situational activation of a goal construct only affects behavior of people for whom that goall is not already chronically accessible (Higgins, 1996).

Based on these findings, we expect emotions to influence behavior only in case a person does not already have a chronically accessible goal that is congruent (i.e, overlaps in terms of action tendency) with the general goal associated with that emotional state. We tested this hypothesis in a one-trial, simultaneous, give-some dilemma game (GSDG).

\section{Chronic goals and emotions in social dilemmas}

Social dilemmas, like the GSDG, can be considered blueprints constituting the prototypical characteristics of an interaction between two (or more) people (players), in which a motivational conflict is presented between a behavioral choice serving one's own interest (defection) or serving the collective interest (cooperation). In the one-trial simultaneous GSDG, the dilemma concems the fact that mutual cooperation has a higher payoff than mutual defection but is also risky, for if one of the players unilaterally defects, he or she will receive the entire profit, whereas the other ends up empty handed (see: Colman, 2003 for an overview). Traditional explanations mainly relied on principles of rational discount, stressing strategic solutions for behavior in social dilemmas. More recent approaches, however, also seek explanations that take into account the psychological factors involved in decisionmaking (e.g., Camerer, 2003). Two such factors are Social Value Onentations (SVOs) and emotions.

SVOS are defined as individual differences in preference for particular outcome distributions between oneself and the other player (MoClintock, 1972): Ustally, two types of SVOs are discemed, pro-social and pro-self. Extant evidence indicates these SVOs to account 
for consistent individual differences in behavior, showing pro-socials to be more cooperative than pro-selves (see: Van Lange, 2000 for an overview). The integrative model (Van Lange, 1999), accounts for these differences by identifying the different goals or motivational tendencies underlying these SVOs (see also: De Cremer \& Van Lange, 2001; Liebrand "Janssen, Rijken, \& Suhre, 1986). When engaged in social interactions, people with a pro-self value orientation only have their self-interest in mind, and their goal is to maximize their own payoff, regardless of the gains to the other player. People with a pro-social value orientation on the other hand, strive to maximize both the joint outcome for both players and the equality of this outcome. Hence, their choices in social interactions are also guided by motivational tendencies towards responsibility (considering the other's payoff) and reciprocity (striving for equal payoffs).

Compared to SVOS, relatively little research has investigated the role emotions play in determining choices in social dilemmas. Studies have indicated that mood influences choice strategies (Hertel, Neuhof, Theuer, \& Kerr, 2000). However, only a few emotions have been studied in social dilemmas. Fear and greed appeared negatively related to the amount of cooperation (Rapoport \& Eshed, 1989), whereas experimentally enhanced feelings of empathy sorted the opposite effect (Batson \& Moran, 1999). Finally; (both naturally occurring and experimentally induced) feelings of guilt, instigated cooperative behavior but only if individuals had previously defected (Ketelaar \& $\mathrm{Au}_{3} 2003$ ).

In two experiments we investigated whether behavioral choice in a GSDG was influenced by experimentally inducing the emotional states of fear (Experiment 1) and guilt (Experiments 1 and 2). Furthermore, we tested whether these influences varied between individuals with different chronic goals (ie, pro-socials and pro-selves).

\section{Experiment 1}

Based on our taxonomy of emotional goals and on results of studies indicating interactive relations between chronic and temporal accessibility of mental constructs, we hypothesized that in a neutral (i.e. non-emotional) state, behavioral choice is determined by chronic goals and that pro-social individuals will be more cooperative than pro-selves. Furthermore, we expected that the impact of emotional states (fear and guilt) would depend on the SVO of individuals. Specifically, fear is associated with the goal to avoid personal risk. Translated to choices in a GSDG, this implies the goal to avoid exploitation or loss. This will result in an action tendency to make a risk-aversive (i.e. a less cooperative) choice, as this is the only means to attain that goal. We expected fear to exert this influence only among pro-socials, however, as pro-selves already chronically strive to avoid exploitation and loss this group. Feelings of guilt on the other hand, activate the goal to make up to someone, which in a GSDG will result in an action tendency to make a more cooperative decision in order not to harm or even benefit the other player. As pro-socials already strive to maximize joint outcomes, guilt priming will only increase cooperation for pro-selves. Hence, we expected interactive effects between SVO and both fear and guilt priming on the amount of cooperation in a GSDG. 
In order to bolster our interpretation of results in tems of Emotion $\times$ Goal - interactions, we started our investigations by explicitly linking SVOs to general goals. Hereto, we assessed differences between pro-socials and pro-selves in importance ratings of values. Values can be regarded as motivational constructs that specify abstract goals guiding people's actions across context and time (Schwartz, 1994). The most popular catalogue of values is Schwartz' Value Survey (SvS), a comprehensive list of ten types of general value constructs differentiated by their motivational goals.

\section{Method}

Participants and Design. A total number of 214 undergraduate students (160 females and 54 males with a mean age of 19.4 years) at the University of Maastricht participated at Time 1. One week later, 131 also participated in the experimental sessions at Time 2. Participants received a monetary reward of $€ 7$, . The experimental design included two between-subject factors, SVO (pro-self vs. pro-social) and Emotion (Fear vs. Guilt vs. Control).

Procedure and Measures: SVO and SVS. At Time 1, we assessed participants' SVOS and administered the SVS in a single session following a lecture. The order of measurements was randomly alternated between participants. Participants were classified as pro-self $(n=82)$ or pro-social $(n=84)$ based on at least six (out of nine) consistent choices on the TripleDominance Measure (see: Van Lange, Otten, De Bruin \& Joireman, 1997, for details). Fortynine participants could not be classified. Participants also completed the Dutch translation of the SVS (Schwartz, 1994). We asked participants to rate the importance of the (56) valueitems as "guiding principles in your dailly life" on a scale ranging from 1 (unimportant) to 10 (extremely important). Previous analyses has indicated that the iterns of the SVS relate to one of 10 types of universal values that can be distinguished on the basis of their motivational goals. As alpha coefficients derived from the present data were sufficiently high (see Table 4.1), item scores for each higher order value type were averaged into single measures.

Priming manipulation. At Time 2, participants came to the lab in same-sex groups of 4 to 6 people. On entering they were welcomed by a female experimenter. They were told that they would participate in two unrelated experiments and were brought to individual cubicles. Participants were randomly assigned to one of the emotion conditions. We used an autobiographical recall procedure (cf. Strack, Schwarz \& Gschneidinger, 1985) to prime emotions. The prime task was introduced as a study on "memory and information processing". Participants were asked to describe "in as much as possible detaill" a recent incident due to which they had felt very much afraid or guilty. Participants in the control condition were asked to describe an average day in their lives. Two investigators, who were blind to the experimental condition, independently judged the adequacy of the priming manipulation based on length and emotional content of the reports.

Cooperation in the GSDG. Subsequently, participants received instructions for the oneshot, simultaneous GSDG (cf. Van Lange, 1999, Study 3). We used a one-shot GSDG to rule out strategic considerations influencing players' choices. The instructions read that each participant would receive four chips representing lottery tickets. (This lottery was actually held at the end of the course.) Participants were given an opportunity to increase their 
number of tickets in an exchange-game that was part of a study on "decision making". They could eam more tickets by a onetime exchange of any desired number of chips (i.e., zero, one, two, three or four) with another participant to whom they would be randomly paired. (We carefully avoided terms (like cooperation, helping, and giving) that could convey socially desirable connotations to any type of choice). Any chips exchanged yielded two tickets to the recipient, but were lost to the donor. Chips kept for oneself yielded just one ticket. A matrix of all possible outcomes of the exchange was provided with the instructions. It was made clear that neither oneself, nor the other participant would know beforehand the number of chips the other had decided to exchange. Participants were told to make sure these rules were clear to them (or otherwise ask the experimenter for extra instruction), before they put the number of chips they wanted to exchange in an envelope, which they handed back to the experimenter along with the instructions.

Control measures. Next, participants were handed another envelope, containing a random number of chips, ostensibly received from the other participant. They also received a short questionnaire asking them to calculate, based on their own and the other participants' payoff, the number of tickets each had eamed. This question served as a measure of understanding of the rules of the GSDG. Finally, after participants were probed for suspicion of a relationship between the priming task and the GSDG, they were thanked for their participation and debriefed.

\section{Results}

SVOS and SVS scores. Preliminary analysis of variance did not reveal order effects on mean importance ratings of values. We investigated differences in mean scores for each of the 10 value constructs of the SVS between pro-selves and pro-socials by a one-way analysis of variance (see Table 3.5). Results indicate that both groups differed on five value constructs. Pro-self individuals considered Power, Achievement and Hedonism values more important, whereas pro-socials rated Universalism and Benevolence values of higher importance. This suggests that the two groups can be well distinguished in terms of their chronically accessible goals.

Cooperation in the GSDG. Participants for whom the priming manipulation had not been considered adequate by both judges, or that incorrectly answered to the questions assessing understanding of the rules of the GSDG, or proved suspicious as to the relation between priming manipulation and GSDG, were excluded from analysis at Time 2. Due to these restrictions, the final sample at Time 2 was reduced from 131 to 75 participants. (These restrictions were also applied in Experiment 2.) 
Table 3.5. Mean Importance Ratings of Value Constructs for Pro-Socials and Pro-Selves

\begin{tabular}{|c|c|c|c|}
\hline Values & $\alpha$ & PSe & PSO \\
\hline Power" & .78 & 5.8 & 5.2 \\
\hline Achievement & .74 & 7.1 & 6.9 \\
\hline Self-Direction & .56 & 7.1 & 7.1 \\
\hline Stimulation & .77 & 6.9 & 6.7 \\
\hline Hedonism" & .74 & 79 & 7.5 \\
\hline Benevolence ${ }^{\circ}$ & .82 & 73 & 7.6 \\
\hline Universalism ${ }^{\circ}$ & .73 & 6.7 & 7.0 \\
\hline Tradition & .62 & 5.4 & 5.6 \\
\hline Conformity & .54 & 6.5 & 6.5 \\
\hline Security & .80 & 6.9 & 6.9 \\
\hline
\end{tabular}

Note. Pse $=$ pro-social; Pso $=$ pro-self.

$+p<.10$, two-talled. $p<.05$, two-tailed. "p $p<.01$, two-tailed.

Following recent recommendations (Abelson \& Prentice, 1997; Judd, McClelland, \& Cullhane, 1995), planned comparisons, rather than the traditional ANOVA procedure, were used to test the hypotheses. The omnibus F-test often lacks power because all possible between-group differences are tested, even those that are not predicted and are thus irrelevant to the study at hand. Using planned comparisons reduces the risk of meaningful differences being overlooked.

The amount of cooperative behavior, indexed by the mean number of chips exchanged, is shown in Figure 3.1. Planned companisons revealed a significant difference, indicating pro-socials $(M=2.21, S D=1.37)$ to be more cooperative than pro-selves $(M=1.00, S D$ $=0.82$ ), $t(75)=2.55, p=.01$ in the Control group, but not in the Fear (pro-social: $M=1.09, S D=$ 0.94 ; pro-self: $M=1.23, S D=1.24, t(75)=-0.30, p=.77$ ), nor in the Guilt-priming condition (prosocial: $M=1.75, S D=0.87$; pro-self: $M=1.67, S D=1.35, t(75)=0.19, p=.85)$. As expected, fear priming significantly reduced cooperation compared to the Control group for pro-socials, $t(75)=-2.43, p=.02$, but not for pro-selves, $t(75)=0.48, p=.64$. Conform our hypothesis, Guilt priming increased cooperation compared to the Control group for pro-selves, but this effect did not reach conventional levels of significance, $t(75)=1.42, p=.08$ (one-tailed). No influence of Guilt priming was found for pro-socials, $t(75)=-1.05, p=.31$

\section{Discussion}

The differences between pro-socials and pro-selves in mean importance ratings on the SVS, corroborate other conceptualizations of SVOS, indicating that value orientations can indeed 


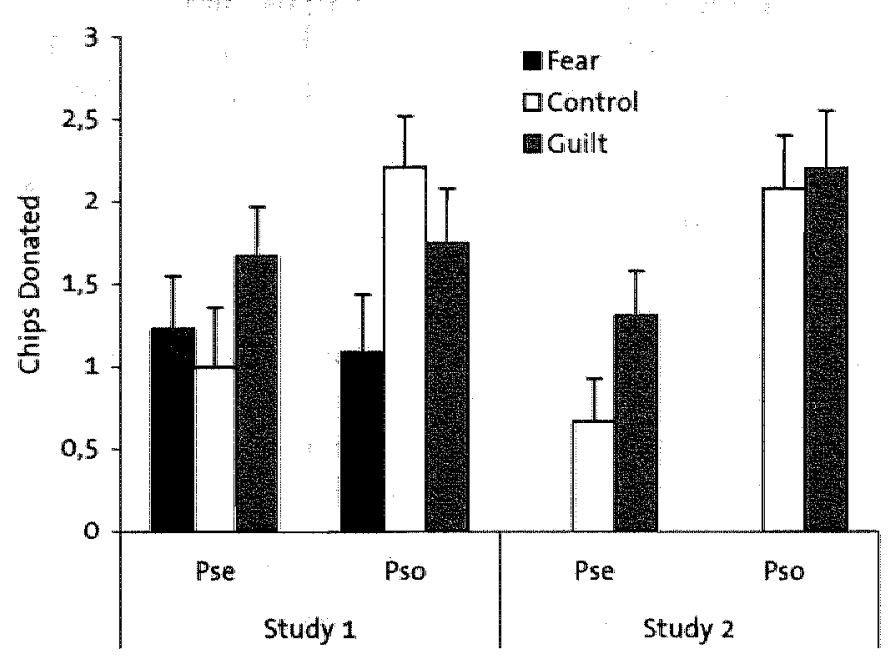

Figure 3.1. Mean number of chips donated $(+S E)$ in Control Fear, and Guilt groups for pro-social (Pso) and pro-self (Pse) value orientations in Studies 1 and 2.

be characterized by differences in chronic goals. First, in accordance with the integrative model of SVOs, the tendency of pro-socials towards reciprocity and responsibility is mirrored by higher ratings on the value constructs of Universalism (related to the appreciation of the equality and welfare of all people) and Benevolence (related to the enhancement of close others). Pro-selves adhere more importance to Power (expressing the pursuit of self-interest) and Achievement values (related to a strive for competence), which reflects the preference of pro-selves to maximize their own outcome and to judge behavior in social dilemmas primarily in terms of might (strength vs. weakness) (Liebrand, et al, 1986). Also, pro-selves rated Hedonism values (related to the pursuit of pleasure and sensuous gratification) more important than pro-socials. This substantiates the finding that pro-socials exercise more personal restraint in social dilemmas (Kramer, MoClintock \& Messinck, 1986).

The results for cooperation in the GSDG supported our main hypothesis to a large extent. In the neutral condition, value onientations determined the amount of cooperation, whereas the influence of emotion priming was different for both value-orientation groups. In line with our expectations, fear reduced the level of cooperation only in pro-socials (who are not chronically motivated to avoid exploitation or loss), whereas guilt increased cooperation only in pro-selves (who have no chronically active goal to take the other player's concems into account). The latter effect, however, did not reach conventional levels of significance. Furthermore, guilt appeared to reduce cooperation among pro-socials, although this effect was also non-significant. Therefore, we conducted another experiment to be positively assured we comectly captured the impact of guilt priming. 


\section{Experiment 2}

In this experiment, we replicated the guilt priming manipulation, using only female participants. Although the data on this matter are not entirely decisive, females have been shown to be more prone to feelings of guilt than men (Tangney, 1990). Therefore, by using female participants we tried to augment the effects of guilt priming. (Due to uneven distribution of male participants between groups, we were unable to investigate this possibility on the data of Experiment 1)

\section{Method}

Participants \& Design. Participants were 67 female undergraduate students at the University of Maastricht (mean age $=21$ years), who received a monetary reward of $€ 7$; - in exchange for participation. The experimental design included two between-subject factors, SVO (pro-social vs. pro-self) and Emotion (Guilt vs. Control).

Procedure and measures. The procedures were similar to those of Experiment 1 with the exception that all data were collected at the same time and that we did not administer the SVS. All measures applied were identical to those in the first study.

Participants came to the lab in groups of 4 to 6 people. On entering they were welcomed by a female experimenter and were given the same cover story before they were seated in individual cubicles. We first assessed participants' SVO (pro-self: $n=37$; pro-social: $n$ $=25$, five participants could not be classified), followed by the priming manipulation (that was again rated for adequacy by two independent judges who were blind to the experimental condition). Subsequently, the instructions to the GSDG were distributed. After participants had handed the instructions and the envelope with the chips they wanted to exchange back to the experimenter, they received the envelope ostensibly containing the chips of the other participant, and a questionnaire asking them to calculate the payoffs to both players in order to control for comprehension of the rules. Finally, after participants were probed for suspicion of a relationship between the priming task and the GSDG; they were thanked for their participation and debriefed.

\section{Results and Discussion}

Planned comparisons again revealed a significant difference, indicating pro-socials $(M=2,08$, $S D=1.44)$ to be more cooperative than pro-selves $(M=0.67, S D=0.84)$ in the Control group, $t(56)=3.48, p=.001$, but also in the Guilt group (pro-social: $M=2.20, S D=1.14$, pro-self: $M=$ $1.31, S D=1.01, t(56)=2.02, p=.05$ ). Compared to the Control group, guilt priming indeed appeared to increase the amount of cooperation for pro-selves, $t(56)=1.72, p=.05$ (one-tailed), but not for pro-socials, $t(56)=0.25, p=.80$.

We subsequently performed a meta-analysis on the influence of guilt priming based on the effect size of the experimental effect in both Experiments 1 and 2. We obtained an overall effect size (Cohen's $d$ ) of 0.65 ( $S D=0.27$ ) for the influence of guilt priming. This exceeds Cohen's criteria for a moderate effect size. The $z$-score for this effect size is 2.41 , indicating an overall significant effect $(p=.001)$ of guilt priming on cooperative behavior in pro-selves. Overall, the results of both experiments thus seem to support our expectation that guilt priming only affects the level of cooperation for pro-selves and not for pro-socials. 


\section{General Discussion}

In two studies we showed that emotional states influence behavior in a GSDG. The effects, however, were not the same for individuals with different SVOs. Fear priming reduced cooperation only for pro-socials, whereas guilt priming increased cooperation only for proselves. We also found that describing pro-socials and pro-selves in terms of differences in chronic goals (as assessed by SVS ratings), match common conceptualizations of these value orientations (e.g. Van Lange, 1999). These results, therefore, may indeed suggest that emotions affect behavior only if a person does not already have a chronically active goal (SVO) that is congruent (in terms of action tendency) to the temporarily activated goal related to the emotional state. Fear is associated with the goal to avoid risk, hence in a social dilemma, to avoid exploitation or loss. This can be achieved by making more defective choices (i.e., to behave less cooperatively). Fear reduces cooperation, however, only if you are not already chronically motivated to do so (ie in case you are not a pro-self). Guilt activates the goal to take someone else's concerns into account, hence increases cooperation, but only if you are not a pro-social, to whom this goal is already chronically accessible. The results thus seem to support our hypothesis that individuals will differ in their (behavioral) response to specific emotions and that this difference is related to individual variation in chronically accessible goals.

The effects of guilt priming were more modest than those of priming fear. Several explanations may account for this difference. First of all, it may be more difficult to prime guilt than fear. Whereas both feelings are negative, guilt is also a self-related emotion. People generally engage in ego-defensive reactions to avoid the profoundly negative state resulting from such feelings (e.g., Lazarus \& Folkman, 1984), which may have diluted the impact of priming guilt. Secondly, functional accounts of self-evaluative emotions, particularly in relation to behavior in social dilemmas (e.g. Frank, 2004), stress that such feelings provoke individuals to forego their immediate self-interest in order to pursue a more effective longterm strategy. Perhaps in a multiple-trial social dilemma (e.g., Ketelaar \& Au, 2003), people would have been more susceptible to guilt As participants in the present studies were aware that they were involved in a onetime exchange, this may have caused them to actively avoid long-tem considerations, thereby counteracting the effect of guilt. Finally, emotions may exert integral or incidental influences. The former concem the influence of experiences that are relevant to the situation at hand (e.g., feeling regret when a gamble has been lost). In our studies, we obviously investigated incidental effects, which refer to influences of feelings that are irrelevant to the present situation. Emotions may differ in their ability to produce such incidental effects. Fear, contrary to guilt, can occasionally be a "free-floating" emotion (Oatley \& Jenkins, 1996), which means its source is not necessarily salient to the person experiencing it. By nature, free-floating emotions may be relatively more likely to produce incidental effects than non free-floating emotions, as people may be more perceptive to the (irrelevant) source of the latter. According to well-established findings, influences of priming dissipate once people become aware that the source of their feelings is irrelevant (Higgins, 1996). This may also account for the weaker impact of guilt. In general, we emphasize that feelings in 
our studies were the result of experimental manipulation and are apt to be relatively weak compared to actual emotions in reallife.

Several limitations of the present studies' must be addressed. First, whereas the results seen to support our hypothesis that emotional influences are moderated by differences in chronic goals; subsequent studies should also explicitly test this mechanism by investigating if a particular emotion indeed enhances the accessibility of associated goals. Secondly, followup research should also exclude altemative explanations of the present results. For instance, the present data could also be accounted for by arguing that pro-socials are more prone to fear, whereas pro-selves are more susceptible to guilt and that as a result, the priming manipulation has differently affected both groups. Finally, it would be interesting to explore interactions for other emotions and goals and in other types of social games (eg. repeated games) or even different behavioral settings.

The renewed appreciation of the pervasive influences of emotions in our daily lives has instigated research resulting in increasingly refined conceptions of the various mechanisms by which emotions affect psychological processes (Schwarz, 2000). For instance, feelings provide a heuristic cue to complex judgments by providing an additional source of information on one's preferences (Schwarz \& Clore, 1996). Recent studies have also demonstrated this influence to extend to specific emotional states (DeSteno, Petty, Rucker, Wegener, \& Bravernan, 2004). In a series of elegant studies, Lemer and Keltner (2001) have shown that emotions also affect judgment and choice in an indirect fashion, by altering the cognitive dimensions along which people appraise a particular situation. The present studies point out yet another mechanism by which emotions pervade psychological processes, ie. by temporarily activating goals. In our opinion, these alternatives do not represent mutually exclusive accounts but rather complementary views in drawing an ever more sophisticated map of our emotional lives.

\section{TO WIND UP...}

In the present chapter, we presented the rationale behind our proposal to use the spectrum of emotions as an index of the fundamental goals underlying human behavior. We discussed theoretical arguments and empirical evidence supporting a functional account of emotions as motivational systems orchestrating a host of psychological processes involved in generating a goal-directed response to the specific demands imposed by the current environment.

We would like to stress that a functional perspective is one among many views on the nature of emotions, and that a certain amount of controversy still prevails on various issues addressed in the present chapter (e.g. Frijda, 2000). At several points we tried to illustrate this debate by discussing opposing views on questions conceming, for instance, the role of cognitive inference required for emotion elicitation, the nature of basic emotions, and the relation between emotions and goals. Nevertheless, providing a detailed image of the 
numerous topics underlying fundamental research on emotions falls well beyond the scope of this dissertation. We limited our discussion to well-documented aspects of emotional processes that were required to ground further assumptions and conclusions, inevitably leaving various interesting matters unaddressed. We focused on the functional perspective as this provided the most hospitable (and solid) ground for realizing our ultimate aim to conceptualize goals in a way that enabled us to account for the impact of specific outcome expectancies as proposed in current EV models.

Given their profound impact on cognitive processes, it seerns palpable that emotions also play a role in shaping the influence of outcome expectancies on behavior. In the subsequent chapter, we attempt to integrate the cognitive and motivational perspectives on behavior by specifying the hypothetical ways in which outcome expectancies are related to emotional processes as just described. We also present several empirical studies testing these hypotheses as well as their potential implications for health-education practice. 


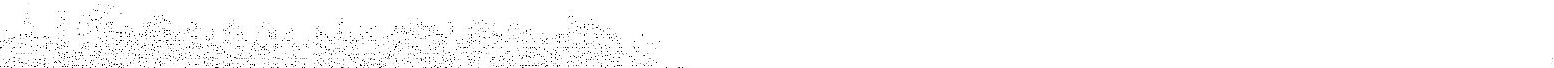




\title{
Chapter 4
}

\author{
Believe What you Want
}

Integrating Motivational and Expectancy $-V$ alue Accounts of Behavior

\section{INTRODUCTION}

In the previous chapter, we outlined the motivational perspective on behavior. Aocording to the motivational perspective, actions are aimed at the attainment of goals. These goals are activated in concert by individual needs and present situational demands or opportunities. A set of general motivational systems guides action to attain these goals, which ultimately originate from universal challenges and opportunities to maximize individual fitness. We pursued the idea that the spectrum of human emotions provides the best index of the nature of these systems and their associated goals. Firstly because emotions are associated with goal-directed action, both in terms of elicitation by signals of goal-relevance, and in terms of motivation, by specifying the general goals for action. Secondly, emotions, as opposed to other goal constructs, are accompanied by bodily sensations, which at an experiential level provide an urge to act, and at an empinical level provide a means to objectively assess physiological correlates of the involvement of emotion in generating behavior. Hence, emotions exceed the level of cognitive representation at which other goal constructs are specified. This, however, does not imply that goals as specified by specific emotions cannot be operationalized by other constructs, like values. 
At first sight it may appear a long leap from this motivational account of behavior to the description of behavioral-control processes as provided by current EV models, which portray actions as the result of volitional choice based on deliberation of expected outcomes. Nevertheless, in the present chapter, we will present an outline for an integrated perspective on behavior by considering the reciprocal relation between outcome-expectancies (OEs) and the operation of motivational systems. In doing so, the motivational impact of OEs, which remains unspecified in current $E V$ models, will be elucidated.

In the following, we will first describe the nature of expectancies, their function in behavioral control processes, and how they relate to the general goal-systems that instigate behavior. The consecutive paragraph starts by specifying how the formation of OEs is essentially a result of the operation of fundamental motivational systems. Given that their roots lie in the experience of outcomes of actions motivated by the fundamental goalsystems; several hypotheses can be formulated on the ways in which future activation of these goals may modulate the impact of specific OEs on behavior.

Two experiments are presented in which these hypotheses were tested. Basically, these studies examined how the behavioral influence of specific OEs (related to the general goals of safety and cooperation) is affected by different levels of activation of these goals. We attempted to allow for the generalization of our findings by manipulating goal-activation through the induction of specific emotional states as well as by semantic goal-priming procedures. Additionally, effects of goal-activation were investigated with respect to different types of behavior, and by applying different experimental designs.

We will stipulate potential implications of the results of these studies for health education practice, before addressing some general issues emanating from the integrated perspective in the final chapter.

\section{THE NATURE AND FUNCTION OF EXPECTANCIES}

In a broad sense, expectancies can be defined as: "Beliefs about a future state of affairs. They are subjective probabilities linking the future with an outcome at some level of probability:" (Olson, Roese, \& Zanna, 1996, p. 211). Following this general conceptualization, virtually all behavior is guided by expectancies. From pushing buttons on the remote to change TVchannels, or wearing a scarf when it's cold outside, to using condoms when having sex; expectancies conceming the effects these actions will have, underlie our behavior. Nevertheless, some organizing distinctions can be made.

\section{Factual and Subjective Outcome Expectancies}

By far the majority of expectancies are generated implicitly. We do not ask ourselves whether pushing a button on the remote might actually result in a change of channels or not before doing so. These implicit expectancies constitute "working assumptions" about the world (cf. 
Olson, et al, 1996). They can be derived from knowledge of the (physical) properties of objects or from enduring experience that a certain act will evoke the same outcome. For instance, our peeling a banana is based on our belief that skin is inedible and we can remove it by tearing it away in strokes. Because we do not question these outcomes, such expectancies can be called "factual expectancies" (of. Olson, et al, 1996). Factual expectancies do not require conscious effort to exert their influence on behavior. Our behavior, like that of most animal species, rests on the assumptions about how the world will react to our actions. These expectancies are the result of the general leaming mechanism allowing our future actions to be based on past experiences of invariant contingencies in the world.

In contrast, many expectancies are held with less than $100 \%$ certainty. These expectancies have been of particular relevance for understanding the behavioral phenomena that have commonly been of interest to social psychologists. For instance, one may believe that eating enough bananas could reduce the chances of getting cancer, but may not be completely sure thereof. Differences in the extent to which people hold a belief like this may underlie the number of bananas they eat every day, hence may correspond to behavioral differences. The assumption that such relevant, yet subjective expectancies are consciously (ie, explicitly) generated to form representations of the possible outcomes of behavior, is the core of the processes described in EV models.

\section{The Function of Outcome Expectancies}

The potential to anticipate outcomes before making a decision to engage in behavior presents a major advantage in terms of behavioral control processes, which has been stressed in various fields of research. It has been referred to as "feed-forward mechanism" in controlsystems theory (eg, Domjan, Cusato, \& Villareal, 2000), as "reasoning capacity" in economical literature (e.g., Hanoch, 2002), and by its more everyday term, "imagination", in (social) psychology (e.g., Olson, et al, 1996). Let's consider this presumed advantage, once more, from an evolutionary viewpoint (cf. Cosmides \& Tooby, 2000):

Behavioral control by motivational systems, as portrayed in the previous chapter, generates flexibility of action beyond that provided by simple decision rules. (Here, flexibility refers to the extent to which behavioral responses are independent from environmental cues (cf. Toates, 1998). The latter constitute a 1-to-1-control mechanism in which the presence of a stimulus always results in a fixed response. Complex environments, however, call for many possible responses, and an integrated set of motivational systems is required to determine the optimal course of action to pursue with respect to the relative importance of the various demands imposed on the organism. Nevertheless such a control system can still only guide behavior in response to the cuies that are currently present in the environment. The capacity to imagine the consequences of possible courses of action, on the other hand, disconnects behavior from cues in the present situation and thereby allows for the planning of actions. Planning adds an additional level of flexibility to behavioral control The benefits of this capacity become obvious from merely considening how it would affect our everyday lives if we were incapable thereof. Going to work whilst still sleepy on a Monday morning, not eating chocolate cakes and chips all day, saving money to go on a long vacation, not hitting 
your annoying colleague in the face every time he bothers you Basically, the enactment of all behaviors involving delay of gratification, or the inhibition of acts providing instant gratification at the expense of later damage involve some form of planning.

\section{The Causal Nature of Outcome Expectancies}

The reader will remember that the search for a system of general goals, which occupied us in the previous chapter, was instigated by the need to relate specific outcome expectancies to the general goals underlying human behavior, in order to account for their motivational capacities. To recapitulate, it was stipulated that causally explaining the occurrence of an action with reference to beliefs concerning its consequences requires an understanding of what causes one to desire a particular consequence (e.g., Bagozzi, 1992; Balleine \& Dickinson, 1998; Frijda, Manstead; \& Bem, 2000; Pervin, 1989).

From the preceding discussion, it should now be clear that such desires arise if the anticipated outcome signals the implications of an act for attaining a particular goal of the motivational systems constituting the ultimate causal agents of behavior. Hence, internal representations of behavioral outcomes have a causal impact on subsequent action precisely because they activate universal motivational systems (i.e, elicit emotional responses) just like actually perceived stimuli do. A host of researchers have explicitly advocated this position (e.g., Balleine \& Dickinson, 1998; Behrendt, 2004; Ben-Ze'ev, 2000; Panksepp, 2000). Damasio, for instance, has argued that emotional responses to anticipated outcomes provide us with a "gut-feeling" (which he refers to as a "somatic marker"). He states: "When the bad outcome connected with a given response option comes to mind, however fleetingly, you experience an unpleasant feeling [a somatic marker, which] functions as an automated alarm signal which says: Beware of danger ahead if you choose the option which leads to this outcome." (1994, p. 174). Similarly, Hanoch (2002, p. 18) argues that: "Without emotions to generate values, reason ... is an arbitrary mechanism lost in a sea of theoretical possibilities and consequences."

Following this idea, an outcome expectancy can thus be defined as: an internal representation of the potential of a particular act to attain or frustrate a general goal.

To summarize, expectancies - conceptions about a future state of affairs - form the basis of nearly all the actions we undertake. Factual expectancies represent behavioral consequences in terms of physical conditions that constitute opportunities and boundaries for motivated behavior. In other words, they determine the form our actions may take. (No matter how hungry we are, we will not eat rocks). Subjective expectancies on the other hand, which are the focus of our interest, represent how behavioral consequences are conducive to or frustrate the attainment of fundamental goals. They causally underlie behavior because they elicit affective / emotional responses similar to environmental stimuli and thus provide a motivational impetus determining the direction of behavior. In the next paragraph we will provide support for the idea that OEs concern the extent to which behavior is conducive to or frustrates the attainment of general goals by exploring the role of general goals in the formation of OES. 


\section{The Role of Fundamental Goals in the Formation of Outcome Expectancies}

In this paragraph we will explore the ways in which motivational systems influence the formation of outcome expectancies. It is generally believed that a person's representations of the world are shaped by experiences from interactions with his (social and physical) environment (Olson, et al, 1996).

Denyberry \& Reed (1994; 1996) approach the topic from a developmental perspective and picture the formation of intemal representations as the result of individual differences in the operation of basic motivational systems. Although they distinguish only between basic approach and avoidance systems, they acknowledge that more specific systems exist that constitute evolved differentiations from more general ones. The authors state that: "whether one chooses to emphasize the general or the specific mechanisms should depend on the domain of inquiry" (Denyberry \& Reed, 1994, p. 659). It is argued that individual differences in the sensitivity of basic motivational systems influences the formation of OEs in two ways. First, by regulation of attentional processes, thus selecting the cues an individual will be exposed and respond to, and hence, determining the nature and scope of objects of which OEs can be formed And secondly, by regulation of behavior, thus determining how an individual will respond to these cues. Different approach and avoidance responses affect the proximity to environmental objects and thereby the type of experience to which an individual is exposed. By regulating behavior, motivational systems thus determine the content of OEs.

Support for their basic idea is derived from a large body of evidence demonstrating how variation in children's representations relates to temperamental differences. (Remember that several authors underscored that variations in dispositional activity of motivational systems underlie personality and temperamental differences.) For example, fearful children may tend to inhibit their approach of novel situations and their attention will favor sources of threat Consequently, their developing representations include the threatening aspects of many objects. The representations of children with a strong approach motivation, on the other hand, emphasize objects as sources of pleasure and reward (Derryberry \& Reed, 1994; 1996).

Frijda and Mesquita (2000) specifically address the potential role of emotions in the formation of beliefs. It is argued that emotional responses to events or objects give rise to beliefs, as emotional reactions entail implicit evaluations (i.e. appraisals) of the meaning or implications of the object or event for the attainment or frustration of the goals that guided the behavior in the first place. Thus: "In that [emotional] experience, the belief is projected upon the object or event as one of its properties" (Frijda \& Mesquita, 2000, p. 52). Consequently, one comes to perceive the neighbors' dog as dangerous because it elicits fear, or starts to believe condoms are frustrating because they reduce sexual pleasure, etcetera.

Of course, some beliefs may wax and wane, lasting only for the duration of the emotional episode Nevertheless, several factors contribute to the development of such momentary experiences into enduring intemal representations (e.g. Frijda \& Mesquita, 2000; Olson, et al, 1996): First of all, direct experience with an object will more likely 
contribute to stable intemal representations of its properties, presumably because beliefs generated from one's own experience are especially trustworthy (see also: Fazio \& Zanna, 1981). Subsequently, repeated experience will contribute to the formation of enduring beliefs, as this constitutes a reaffirmation of the objects' properties. Finally, a belief may acquire constancy because of the emotional costs involved in abandoning it, such as is the case with beliefs held to conform to a social group (Frijda \& Mesquita, 2000).

Summanizing specific associations may develop because motivational systems influence attention and guide behavior so that the formation of certain representations concerning the properties of objects and events becomes more likely. Furthermore, emotional responses to outcomes of interactions between individuals and their ervironment, by nature, entail information conceming the implication of objects and events for the realization or frustration of fundamental goals. As the operation of motivational systems underlies the formation of $\mathrm{OES}$, it thus seems reasonable to expect that outcome expectancies comprise the consequences of behavior for the attainment of a particular goal.

In the following paragraph we will address the implication of these links between OEs and goals for the relation between OEs and behavior. Specifically, we will outline how differences in the extent to which particular goals are activated in a decision situation may affect the impact of OEs on behavior. Two studies will be presented in which these assumptions are tested. We will further illustrate how health edication practice might utilize the goal-based nature of outcome-expectancies.

Notwithstanding these objectives, we do not mean to imply that OEs do not have impontant implications themselves: Undeniably, individual differences in (the extent to which people hold) particular OEs, have profound cognitive, affective, and behavioral consequences (Olson, et al, 1996). Such effects constitute the basis of EV account of behavior and have been widely demonstrated. The objectives of the present research, however, concem the consequences of temporary goal-activation that is not premeditated by an individual in the process of intention formation.

\section{EFFECTS OF GOAL-ACTIVATION ON THE IMPACT OF OUTCOME EXPECTANCIES ON ACTION}

In general, we expect emotional states to affect the behavioral influence of specific OEs that entail consequences of performing the act for attainment or frustration of the general goal associated with the emotional state. Given the profound impact of affective and specific emotional states on cognitive processes as described in the previous chapter, proposing an impact of emotions on the cognitive processes that mediate the impact of outcome expectancies on behavior as described in EV models, may come as no surprise. Emotions can alter the relation between OEs and behavior in several ways (see Figure 4.1). Firstly, emotions may result in changes in accessibility or evaluation of particular OEs, either in terms of 
likelihood, valence, or both Furthemore, emotions may change the impact a specific Or has on behavior (ie, moderate the relation between outcome expectanotes and behavion). In the following we will elaborate on these proposed influences.

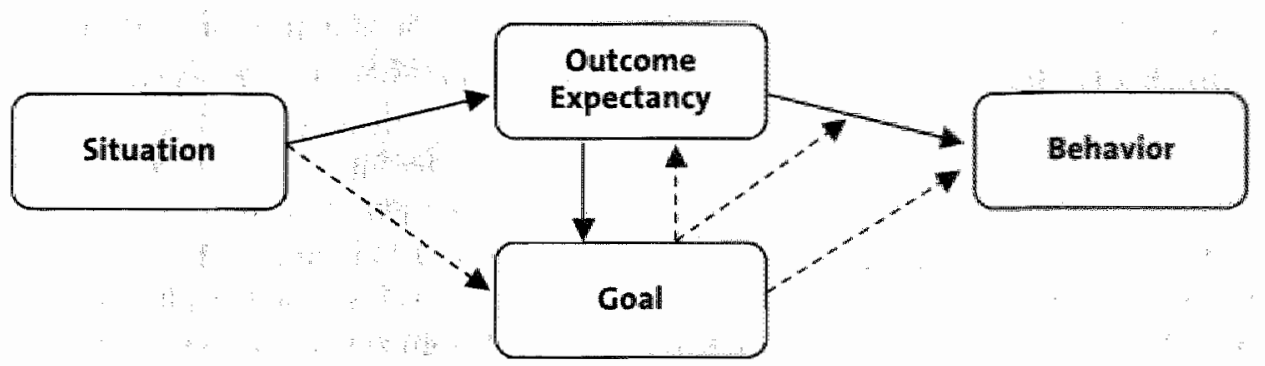

Figure 4.1. Schematic representation of the processes of behawioral causation according to the integrated perspective. Solid lines depict the processes as described in current EV models whot whe acknowledgement of the goal-related nature of motiwationally salient OEs. Dasthed hines pheture the hypothesized impact of goal-activation on OE - Behavior relations; and the ctirect ingactof goat arctivation on behavior.

\section{Emotional Influences on the Accessibility of Outcome Bxpectancies}

Emotion-specific effects on accessibility of OEs (Hypothesis 1) initially follows from Tulving's (1983) principle of encoding specificity, which states that information is more readily retrieved from memory if an individual is exposed to cues identical as present during encoding of the information As described in Chapter 3, extant evidence on mood-congruent memory retrieval (e.g. Bower, 1981; Forgas, 1995) attests to this principle. According to the integrated perspective, OEs are associated by common goals. Consequently, it can be expected that in a particular emotional state, goal-related OEs will be more accessible to people than non-relevant $\mathrm{OEs}$, and therefore, have greater impact on behavior. Indeed, more accessible constructs in memory are generally found to exert greater impact on behavior than less accessible constructs (Higgins, 1996).

\section{Emotional Influences on Expectancy and Value Estimates}

Emotions may also influence people"s ratings of anticipated outcomes both in terms of likelihood (Hypothesis 2) and value (Hypothesis 3). The latter may be somewhat obvious as preferences (ie, values) to certain outcomes are by definition related to the extent to which the outcome is desired. The hungrier I am, the more positively I will evaluate having lunch or any other meal. Similarly, if I feel afraid or concerned for my own safety, I will be more positive in my evaluation of a condom's perceived ability to prevent me from getting HIV, and when I"m very much sexually aroused, the expectation that putting on a condom will spoil my pleasure will seem more negative to me.

Conceming the expectancy component, much work has demonstrated moodcongruent influences on likelihood estimates of similarly valued events. Specifically, research 
on risk perception (eg, Johnson \& Tversky, 1983), has shown how a negative mood inflates frequency estimates of negative events (e.g., tomadoes, instances of leukemia, violent acts) in general Simillarly, positive events appeared more probable to happy people. According to The Affect as Information Model (Schwarz \& Clore, 1996, see also Chapter 3), these effects occur because affective states (whether relevant to the present situation or not) serve as informative cues regarding the general status of one's environment. Hence, negative mood is associated with greater endorsement to views of the environment as a bad place in which negative events are likely to happen (see also: Forgas, 1995). Recentevidence, however, seems to indicate such mood-congruent effects on likelihood estimates to extrapolate to specific emotional states as well Specifically, experimentally induced emotional states like anger, fear, and sadness appear to influence likelihood judgments of events with similar emotional implications (DeSteno, Petty, Wegener, \& Rucker, 2000; Lemer \& Keltner, 2000; 2001). These results suggest that emotional states may also affect the likelihood estimates of outcome expectancies conceming the vanious consequences of specific behavioral acts.

\section{Emotional Influences on OE - Behavior Relations}

Emotions may also influence the relation between OEs and behavior (Hypothesis 4). Specifically, behavioral decisions may be differentially affected by certain anticipated consequences, dependent upon an individual's emotional state. For instance, considerations concerning the abilities of condoms to protect from getting sexually transmitted diseases, will be more important to a person who is more concerned with his own safety (ie., health) than to a person who's main concern is the sexual pleasure he has while having sex. To the latter individual, beliefs regarding condoms as diminishing sexual pleasure will be more important determinants of behavior. As such, the impact of outcomes on behavioral choice is dependent upon the relative importance of the goals underlying this decision. In other words, emotions may moderate the impact of an $O E$ on behavior, inrespective of accessibility, likelihood and value ratings.

This prediction follows from what Frijda (e.g. Frijda \& Mesquita, 2000) calls: "concern relevance", meaning the implications of an outcome for the goal(s) an individual is currently pursuing. Similarly, Olson et al, (1996) refer to the "motivational significance" of an OE as a determinant of its importance for behavior. As outcome expectancies specify behavioral consequences with reference to fundamental goals, an emotional state - considered a temporal increase in the activity of such a general goal (cf. Roseman, Wiest, \& Swartz, 1994)will increase the importance of an $O E$ that is of relevance to this particular goal, as a determinant of behavior.

\section{Previous Research on Affective Influences on OEs}

So far, only a handful of studies have examined the influence of affective states on the relations between OEs and behavior. Notably, these studies have exclusively addressed the influence of general (positive or negative) mood states on the likelihood and accessibility of OEs. For either, results are equivocal. 
Hufford (2001) investigated the impact of mood induction on the endorsement (i.e, perceived likelihood) of positive outcomes related to alcohol consumption. He found negative mood to enhance the endorsement of positive outcomes. Positive mood, on the other hand, had no influence on the perceived likelihood of outcomes. Rather than supporting mood-congruent likelihood estimates, these results were interpreted to support predictions from coping-theory (Carver \& Scheier, 1990), which predicts that negative affect will facilitate endorsement of positive outcome expectancies to promote attempts at mood repair. In contrast, Cohen and colleagues (Cohen, McCarthy, Brown, \& Myers, 2002), found positive but not negative mood to result in increased likelihood ratings of positive outcomes related to smoking. Furthermore, these likelihood ratings appeared to partially mediate the main effect of mood on behavioral intentions. The results of this study did not support a moderating influence of mood state on the impact of likelihood ratings on behavioral intentions, hence, denying a direct influence of affective states.

Two studies investigated mood influences on the accessibility of OEs. Both examined this influence by coding the type of the first in a set of self-generated outcomes that individuals reported with respect to a particular behavior. McKee and colleagues (McKee, Wall, Hinson, Goldstein, \& Bissonnette, 2003) showed that positive mood induction enhances the accessibility of outcomes related to the positive reinforcing aspects of smoking (e.g. stimulating experiences). They also claim that individuals in a negative or neutral mood are more likely to mention negative reinforcing consequences (e.g, tension reduction), but as the negative and neutral groups do not differ, this result is probably more accurately interpreted as the positive mood state reducing the accessibility of these consequences. Indeed, in another study, positive mood induction was also found to enhance accessibility of positive outcomes related to alcohol consumption (e.g. social enhancement) but to reduce accessibility of negative reinforcing outcome expectancies (Goldstein, Wall, McKee, \& Hinson, 2004).

To summanize, whereas theoretical and empirical evidence may support expectations concerning emotional influences on OEs, so far, studies have mainly addressed general mood effects and have moreover, yielded inconsistent evidence.

\section{STUdY 4.1: EMOTIONAL INFLUENCES ON PERCEIVED OUTCOMES}

We tested the predictions concerning the influence of specific emotions on $\mathrm{OE}$ - behavior relations in the context of people's considerations and intentions to interfere with random violence. Specifically, we assessed emotional influences on two OEs, capturing the most conspicuous considerations involved in decisions to interfere with random violence (cf. Fritzsche, Finkelstein, \& Penner, 2000): One specifying the personal risks involved, related to the general goal of fear, the other focusing on the beneficial consequences of helping for the victim, congruent with the general goal of guilt. We expected that inducing fear, compared to a neutral control group, would enhance the accessibility of harmful personal consequences, 
boost the perceived likelihood thereof, or make individuals more negatively evaluate this consequence. Similar effects were predicted for the influence of guilt priming on the anticipation of beneficial consequences for the victim. Notice that we do not claim that the consequences in terms of accessibility, likelihood, and valence are mutually exclusive:

We also assessed participants intentions towards interfering when witnessing an act of random violence. In general we expected that fear priming would result in lower intentions towards interfering (compared to a control group), whereas guilt priming would show the opposite effect. Furthermore, we tested whether emotional states moderated the influence of associated OEs on behavioral intentions. We expected that personal consequences would be more important determinants of intention in the fear, compared to the control group, whereas considerations conceming the victim would more strongly influence intentions in the guilt, compared to the control group.

\section{Method}

Participants \& Design. A total number of 109 undergraduate students at the University of Maastricht participated in the study. After exclusion of subjects for whom the priming manipulation was considered inadequate $(n=15)$ or proved suspicious to the relation between priming and measurement of intentions and $O E s(n=9), 85$ participants ( 72 females and 13 males with a mean age of 19.3 years) were included in the analyses. Participants received a monetary reward of $€ 5$,. The experimental design included one between-subject factor, Emotion-group (Control vs. Fearvs. Guilt).

Procedure and Measures. After a lecture, we obtained a measure of participants" baseline intentions to intervering with random violence, which was embedded in a questionnaire assessing people's willingness to engage in a number of ostensibly cooperative acts. The baseline intention measure $(M=3.4, S D=1.4)$ asked participants to indicate the extent to which they would be inclined to: "Physically interfere to end the conflict when you would witness an act of random violence", on a 7-point scale, ranging from 1 (definitely not) to 7 (definitely).

During the experimental sessions, approximately one week later, participants came to the lab in groups of six people. On entering they were welcomed by a female experimenter. They were told that they would participate in two unrelated studies, and were brought to individual cubicles. An autobiographical recall procedure (cf. Strack, Schwarz \& Gschneidinger, 1985) was used to prime emotions. The task was introduced as a study on: "memory and information processing". Participants were asked to describe, "in as much as possible detail", a recent event due to which they had felt very much "afraid" or "guilty". The Control group was asked to describe: "an average day in your life". Two experimenters; who were blind to the experimental condition, independently judged the adequacy of the priming manipulation based on length and emotional content of the reports.

Subsequently, participants completed a questionnaire, measuring intentions and OES on interfering with random violence. Measurement order was altemated, with approximately half of the participants $(n=39)$ first completing intention measures, and the 
other half $(n=46)$ first completing $O E$ measures. All questions were presented and rated on a computer screen. As instances of random violence may greatly differ, we tried to make sure participants had the same image of the situation in mind before answering the questions. Hereto, we presented a scenario, which they were told to read carefully while "imagining you were actually confronted with the situation described". The scenario pictured the reader, walking home alone at night through empty streets when suddenly witnessing two guys beating up another guy. After reading this scenario, which was presented by an automatized power-point presentation to ensure equal reading-times, participants completed the questionnaire.

Intention Measures. Participants' intentions were assessed by asking them to indicate their initial response in case they encountered the situation as just described to them by moving a cursor to a point on a Visual Analog Scale (VAS) and clicking the mouse-button if they were convinced the cursor position accurately indicated their intention. Scale end-points were labeled "leave the situation" and "physically interfere". The distance from the center of the VAS to the point indicated by the participant was recorded by the computer and was assigned an integer value between -50 and 50 , with more extreme positive values indicating stronger intentions towards physical interference, and more extreme negative values indicating stronger inclinations to leave the situation.

OE Measures. We assessed accessibility, likelihood, and evaluation of two OEs, one related to fear (OEf: "If I physically interfere in the situation described, I nun the risk of becoming a victim myself), and one related to guilt (OEg: "If I physically interfere in the situation described, I will be able to help the victim"). Both OEs; along with two filler-items unrelated to fear and guilt, were presented twice on a computer screen in randomly altemating order. Participants were instructed to indicate as fast as possible whether they considered each outcome "likely" or "unlikely", by respectively pressing either the 'p' or the ' $q$ ' key on a standard OWERTY keyboard Accessibility was measured by recording the (average) response time (RT) for decisions on each of the items (cf. Naumann \& Richter, 2000)4. Following guidelines proposed by Fazio (1990), raw RTs were transformed (1/X) in order to approximate them to the normal distribution. Furthermore, all RTs outside \pm 3 sd's of the population mean for each item were recoded to the values representing the (upper and lower) boundanies of that range. We subsequently assessed participants' ratings of likelihood and evaluation for both items on 7-point scales, ranging from -3 (very unlikely / very negative) to 3 (very likely / verypositive).

After they finished the questionnaire, participants were probed for suspicion of a relation between the priming task and their responses on the questionnaire items. Finally, they were debriefed, paid, thanked for their participation, and dismissed.

\section{Results}

OE Measures. In order to exclude confounding by intention scores, we analyzed differences between Emotion-groups only for participants who first completed OE measures. Mean differences in accessibility, likelihood, and evaluation (see Table 4.1), as well as EV 
scores (obtained from multiplying likelihood and evaluation ratings) between Emotion groups were investigated by a one-way analysis of variance. Results did not indicate significant differences between Emotion groups in mean RTs for either OEf, $F(2,43)=.88, p=$ 42 , or OEg, $F(2,43)=46, p=63$. Nor did expectancy ratings (OEf, $F(2,43)=23, p=80 ; \mathrm{OEg}$, $F(2,43)=61, p=55$ ) or evaluations (OEf, $F(2,43)=1.96 ; p=15 ; \mathrm{OEg}, F(2,43)=.16, p=.85$ ) differ between experimental groups. Finally, results on EV products did not differ either (OEf, $F(2,43)$ $=1.26, p=30 ; \mathrm{OEg}, F(2,43)=1.01, p=37$ ). Analyzing all cases yielded similar results.

Table 4.1. Mean likelihood ratings, evaluations, and accessibility of Control, Fear, and Guilt groups on Outcome Expectancies related to Fear (OEf) and Guilt (OEg)

\begin{tabular}{|c|c|c|c|c|c|c|}
\hline & \multicolumn{2}{|c|}{ Likelihood } & \multicolumn{2}{|c|}{ Evaluation } & \multicolumn{2}{|c|}{ Accessibility ${ }^{2}$} \\
\hline & OEf & OEg & OEf & OEg & OEf & OEg \\
\hline Control & 1.26 & 1.53 & -163 & 2.74 & 1014.5 & 866.0 \\
\hline Fear & 173 & 2.00 & -282 & 2.64 & 1193.5 & 873.0 \\
\hline Guilt & 1.44 & 1.88 & -2.00 & 2.56 & 12720 & 980.0 \\
\hline
\end{tabular}

afor clarity, average response latencies in ms. are reported to index accessibility.

As we did not find emotion induction to influence accessibility of $O E s$, this may indicate that RTs reflect chronic individual differences in the association strength of perceived outcomes and behavior (eg.. Fazio, 1990; Higgins, 1996; Olson et al, 1996). We therefore performed a median-split on participants' RTs for each outcome to differentiate between subjects for whom the particular outcome was strong vs. weakly associated with the behavior. Following recent recommendations (Abeison \& Prentice, 1997; Judd, McClelland, \& Culhane, 1995, see also Study 3.2), planned companisons were used to test if emotion induction differentially affected EV ratings of OEs for people with strong vs. weak associations (see Figure 4.1).

Concerning the fear-related outcome, results indicated that for people with strong associations, EV scores did not differ between the Control $(M=-5.6, S D=14)$ and neither the Guilt $(M=-5.6, S D=2.0 ; t(37)=.00, p=1.0)$ nor the Fear group $(M=-4.4, S D=1.7 ; t(37)=-.53, p=$ 60). For people to whom this outcome was only weakly associated with the behavior, however, results indicated that the Control Group $(M=11, S D=17)$ differed significantly from the Fear $(M=-6.0, S D=2.2 ; t(37)=2.6, p=.02)$, but not from the Guilt group $(M=-2.6, S D=$ $1.5 ; t(37)=1.7, p=11)$. Similarly, EV ratings of the guilt-related outcome, did not differ between the Control $(M=6.0, S D=0.9)$ and neither the Guilt $(M=6.8, S D=1.1 ; t(38)=-.51, p=$ 61) nor the Fear group $(M=7.7, S D=1.2 ; t(38)=-1.1, p=.27)$ if strongly associated with the behavior. For people with weak associations, however, results indicated that the Control Group $(M=2.1, S D=1.2)$ differed significantly from the Guilt $(M=5.7, S D=1.2 ; t(38)=-2.1, p=$ .04) but not from the Fear group $(M=2.3, S D=1.6 ; t(38)=-.05, p=.96)$. 


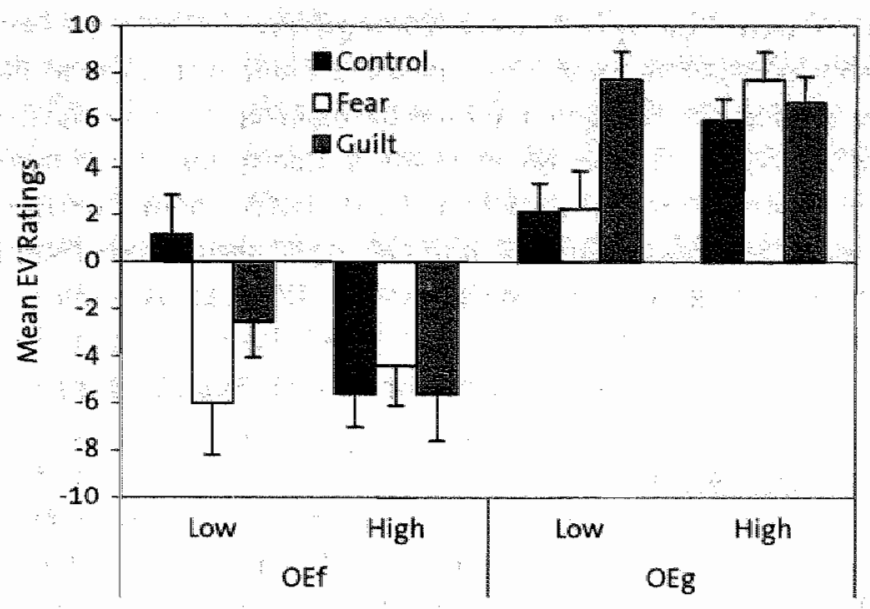

Figure 42. Mean EV Ratings (45) for Control, Fear, and Guilt groups on High vs. Low Accessible Expectancies nelated to fear (OEf) and Guilt (OEg)

Intention Measures. Average ratings on the VAS-scale indicated participants were not substantially inclined towards interfering or running away $(M=2.3, S D=16.4)$. To investigate influences of emotions on $\mathrm{OE}$-behavior relations, we tested whether emotional state moderated the influence of each OE on intention scores.

Table 4.2. Summary of Hierarchical Regression Analysis of Variables Predicting Intentions to Interfere with Random Violence

\begin{tabular}{|c|c|c|c|}
\hline Variable & $B$ & SEB & $\beta$ \\
\hline \multicolumn{4}{|l|}{ Step 1} \\
\hline 10 & 2.84 & 1.2 & $.26^{*}$ \\
\hline Fear & $\therefore \quad-6.06$ & 4.0 & -18 \\
\hline Guilt & $: 4$ & 3.7 & .00 \\
\hline OEf & 41 & .3 & .16 \\
\hline OEg & $\quad 00$ & .5 & .00 \\
\hline \multicolumn{4}{|l|}{ Step 2} \\
\hline Fear X OEf & .71 & .8 & .13 \\
\hline Guilt $\times$ OEf & .64 & .7 & 16 \\
\hline FearxOEg & -1.10 & 1.3 & -.21 \\
\hline Guillt $\times$ OEg & .43 & 12 & .09 \\
\hline
\end{tabular}

Note. $h_{0}=$ Baseline Intention; $\mathrm{OEf}=$ Fear-Related Outcome Expectancy; OEg $=$ Guilt-Related Outcome Expectancy $R^{2}=13$ for Step 1; $\Delta R^{2}=.03$ for step 2 (ns): p $<.05$. 
Moderation means that the impact of a variable (in our case EV scores of OEs) on an outcome measure (intention) differs across levels of another (ie, the moderator) variable (Emotion group). A hierarchical regression procedure was used to demonstrate moderation of $O E$ - Intention relations by emotion (cf. Baron \& Kenny, 1986). We regressed intention scores on baselineintention measures, fear and guilt (which were dummy-coded), and EV ratings for each OE separately (Step 1), and subsequently entered interaction temis of emotion and OE (Step 2). Results are summarized in Table 42. As none of the interaction terms of emotion and $\mathrm{OE}$ received significant Beta-values, the models did not indicate emotional state to moderate the influence of OEs on intention (which themselves were also non-significant).

Finally, we investigated whether intention scores (corrected for baseline intention) differed between experimental groups. Again, planned comparisons were used to test for between-group differences. Results did not reveal differences between the Control $(M=20$, $S D=15.6)$ and the Guilt group $(M=1.5, S D=14.7 ; t(8.2)=.14, p=.89)$. The Fear group, however, reported lower mean intention ratings $(M=-4.8, S D=12.2)$ than the Control group, $t^{\prime}(82)=1.8$, $p=.04$ (one-sided), but did not differ from the Guilt group, $t(82)=1.5, p=.12$.

\section{Discussion}

The data provided limited support for some of the hypothesized consequences of emotion induction (ie., the activation of general goals) on the evaluation of goal-congruent OEs. Although neither likelihood, nor value ratings were uniquely affected by emotional state, we did find indications that emotions influence the combined EV products of goal-congruent OEs. These effects were confined, however, to anticipated outcomes that were not highly accessible to people (ie., that were not strongly associated with the behavior under investigation).

A parallel can be drawn with the results of Study 3.2, in which emotions were found to sort effects (on behavioral choice) only if people did not already have a chronically salient, situation-specific behavioral goal guiding their decisions in the same direction as the general goal associated with the emotional state. Similarly, the results of the present study can aiso be accounted for by interactive effects of chronic and temporary-enhanced (ie., due to priming) accessibility of mental constructs (Higgins, 1996). As OEs specify a general goal in a behavior-specific way, the results of the present study imply that emotions change the expected value of behavior for goal attainment, only if individuals do not already strongly perceive behavior as conducive to the attainment of this particular goal Nevertheless, the status of this interpretation should be considered preliminary for it relies on a post-hoc classification of OEs as high vs low in accessibility, only after we ascertained that emotional state did not affect the accessibility of outcomes. In order to be sure, outcome accessibility should be determined before priming emotions or other means of activating goals.

Obviously, these results do not allow for strong conclusions to be drawn conceming the impact of emotions on EV-processes. Nevertheless, they do seem to contradict the primary altemative view, which would hold that emotions do not exert an influence whatsoever. 
Such a view would imply that beliefs, though once established by an emotional response, eventually operate independently of motivational state, ie., acquire "functional autonomy" (cf. Allport, 1935). The finding that emotional state affects EV ratings of less accessible OEs shows that anticipated outcomes are not fully autonomous in this sense and that the data are better accommodated by a connectionist perspective (eg., Anderson, 1983), in which the salience of $O E s$ is a cognitive event, triggered by situational cues (i.e. emotions). According to connectionist models, intentions are a composite state of (temporary) aroused beliefs, and different belief-sets may underlie intentions on varying occasions (e.g. Lowe, Bennett, Walker, Milne, \& Bozionelos, 2003). Various authors (e.g., Fazio, 2001; Ferguson \& Bargh, 2003) attest to the view that mental constructs, like intentions and attitudes, rather than exclusively constituting readily stored and retrievable components of memory, are sometimes better perceived as constructions formatted on-the-spot by automatic activation of relevant cognitions. Fazio's (e.g., 1986) research in particular has demonstrated how association strength (e.g. between an intention and a perceived consequence of acting) is dependent upon the extent of previous activation (e.g., due to prior experience with behavior). Similarly, Schwarz and Clore (1996) have pointed out that it is particularly in the absence of clear knowledge, that emotions are apt to be used as sources of information. In line with this view, emotions seem to only affect the evaluation of weakly associated consequences. As we did not assess the amount of prior experience with random violence, however, we could not determine whether this influenced the accessibility of OEs. Note that connectionist perspectives are not necessarily at odds with standard EV interpretations of intention formation (Eagly \& Chaiken, 1993; Eagly \& Chaiken, 1998); yet do put a stronger emphasis on situational (external) factors that may modulate this process.

As people's ratings of OEs did not significantly predict their intentions to interfere with random violence, we were unable to test whether $E V$ ratings mediated an impact of emotion on intention. Probably, a more exhaustive set of anticipated outcomes of interfering with random violence would have been required to reliably predict intentions. Moreover, other factors, beside OEs, determine people's intentions, like self-efficacy for instance. Although our primary interest concemed the impact of goal-activation on the evaluation of OEs, future research should investigate a more comprehensive set of determinants of intention and behavior in order to investigate whether emotional influences on OEs actually result in behavioral differences.

Furthermore, the data did not support the hypothesized influence of goal-activation on the relation between OEs and behavior, as emotional state did not appear to moderate the impact of EV ratings on intention measures. It has often been remarked, however, that in general, additive models provide an adequate description of social-psychological data (e.g., Evans, 1991), and that consequently, statistical support for multiplicative models (i.e., with interaction terms) is hard to obtain.

In addition, several factors - some of which related to limitations to the design of the present study - may account for possible attenuation of the hypothesized impact of emotions on the evaluation of anticipated behavioral consequences and their relations with 
behavior. First of all, complications may arise in assessing clear-cut relations between emotions and specific OEs due to idiosyncratic differences in the framing of behavioral outcomes. Rerognition must be given to the idea that the information people store about behavioral outcomes does not necessarily take the form of propositional (ie, if - then) statements (Dijker, 2001; Eagly \& Chaiken, 1993). Therefore, individuals are likely to vary in the framing of sich outcomes, which places strong demands on the wording of items as approximation of the actual individual representation of an outcome. For instance, whereas one may conceive of the personal nisks from interfering with random violence as "causing rne to become a victin myself", another may more generally think of it as "running a nisk", or more specific as "getting a beating", which nevertheless ultimately refers to the same consequence. Hence, the phrasing may cause the same OE to more closely resemble the representation for some individuals than others, diluting influences of goal priming on people's evaluation of the outcome. We wish to emphasize that other studies investigating affective influences on accessibility or evaluation of expectancies have either used validated questionnaires (e.g., Hufford, 2001, Cohen et al., 2002) or sets of self-generated beliefs (e.g; Mckee, et al, 2003, Goldstein, et al, 2004). Both approaches seem more likely to capture potential consequences in a way that closely resembles the (average) respondent's owm conceptualization thereof, which makes it more likely to tap the effects of modulating infuences on these items. Furthermore, the study by Desteno and colleagues (2000) - the only one to show influences of specific emotions (rather than moods) on likelihood estimations - did not assess such effects on behavioral outcomes, but rather in terms of frequency estimates of events. For instance, it was found that an induced emotional state (e.g, anger), increased frequency estimates of a hypothetical event (e.g the number of criminals that will be set free because of legal technicalities) arousing the same emotion, but not of an event (e.g., the number of people that would expenience the death of a loved one) that would arouse another emotion (e.g, sadness). Perhaps frequency estimates of abstract events that are of lesser personal relevance than an outcome of behavior in which oneself is supposedly engaged in are more susceptible to emotional influences than hikelihood ratings of outcomes of one's own behavior.

Secondly, investigating incidental (ie, non-relevant) emotional influences is apt to encounter difficulties, as specific emotions often have a specific focus and therefore do not transcend easily to other objects (as was also mentioned in the discussion of the results of Study 3.2). This argument would apply for guilt more readily than for fear priming, as the latter is occasionally "free-floating" (cf. Oatley \& Jenkins, 1996), that is, without a specific object. Indeed, effects of fear priming were stronger than of guilt priming, both in terms of EV ratings of (weakly) associated OEs as well as behavioral intentions. Note that other stiudies have investigated the influence of mood states on outcome evaluations and accessibility. which are more likely to evoke incidental influences on cognitive judgments:

Finally, emotions may exert a direct influence on behavior. Indeed, the results of the present study show that fear priming results in lower intentions to physical interference with random violence. This effect, moreover, was not mediated by participants ratings of the 
fear-related anticipated outcome. The presence of such direct influences does not only imply that emotions are less likely to affect the evaluation of OEs, but may even diminish the influence of anticipating outcomes in the formation of intentions altogether (Loewenstein, Weber, Hsee, \& Welch, 2001).

In conclusion, the results of the present study imply that emotions may influence relations between outcome expectancies and behavior by changing people's expectancy value ratings of goal-congruent outcomes, to the extent that such outcomes are not already strongly associated with the behavior. Nevertheless, as no support was found for each of the specific hypothesized influences of emotions (i.e., on expectancy or value ratings exclusively or by moderation of the $\mathrm{OE}$ - behavior relation), the data does not allow for the inference of the particular process underlying this effect.

\section{Study 4.2: Goal-Based Matching of Persuasive Messages}

In order to allow for a more detailed evaluation of proposed emotional influences on EV processes, we performed another experiment, adopting a different design to also highlight some of the implications of our theoretical perspective for health education practice. In Study 4.1 we investigated the influence of induced emotional states (ie, the activation of general goals) on people's evaluations of (supposedly) existing outcome expectancies and their impact on behavior. In this study, however, we examined whether differences in prior activation of general goals would result in goal-congruent differences in (a) the evaluation of induced OEs (ie, by persuasive messages) and (b) the extent to which these OEs affect behavior.

Apart from bearing more relevance to health education, the present study circumvented some of the limitations of the previous one. First of all, influences of individual differences in accessibility of OEs were excluded, as the topic of the present study (a genetically modified banana) was fictitious and thus unfamiliar to all participants. And secondly, by introducing a novel topic, we limited the possible distortion of goal-activation effects by individual differences in the framing of behavioral outcomes. Moreover, the present study extended the previous one, by applying another type of priming manipulation to activate the general goals of safety and cooperation, and by incorporating a behavioral measure.

\section{Previous Research on Goal-Based Matching of Persuasive Messages}

The basic hypothesis behind this study is that activation of a general goal will determine the impact (on behavior) of those elements of a persuasive message that specify consequences to the attainment or frustration of that goal. Following Hypotheses 2 and 4 respectively, we expected this effect would either be caused (ie, mediated) by the increased endorsement (ie., perceived likelihood) of the consequences mentioned in the message that are relevant to the activated goal, or due to moderation by goal state of the impact of goal-congruent outcomes on behavior. 
The general position that a match between certain characteristics of a receiver and the source or content of a message enhances persuasion is not a new one (see: O'Keefe, 1991, for an overview). Several lines of research in this area have specifically focused on matching persuasive messages to individual differences in goal-related aspects of attitudes and behavior. For instance, research on attitude-function addresses the psychological purposes or needs served by holding a particular attitude (for overviews, see: Eagly \& Chaiken, 1998; Maio \& Olson, 2000c). The basic tenet of the functional approach to attitudes is that people hold and express attitudes for different reasons, and that knowledge of this motivational basis is the key to attitude change. Basically, research in this domain has documented that messages that are matched to individual (e.g. Snyder \& DeBono, 1985) or temporarily induced differences in induced goals (Julka \& Marsh, 2000), are more effective in inducing attitude change than mismatched messages.

Early theories on attitude function (e.g., Katz, 1960; Smith, Bruner \& White, 1956) discerned five seminal functions or goals of attitudes. More recent perspectives, however, suggest that several goals derived from individuals' values and needs may underlie people's attitudes (Maio \& Olson, 2000a; 2000c). For instance, priming different goals (being successful and enjoying life) resulted in differences in susceptibility to messages persuading to donate money either to a study skills program or a travel fund (Maio \& Olson, 2000b). Although both values related to utilitarian attitude function in early conceptions, they nevertheless produced different effects on people's attitudes towards donating; suggesting that the goals underlying people's attitudes (and behavior for that matter) are more differentiated that initially proposed.

Other studies have provided additional support for the basic idea that matching persiuasive messages to the general goal state of individuals increases their effectiveness in terms of attitudes and behavior change. For instance, thirsty participants in a study investigating ads for different sports-drinks indicated greater preference for a drink after reading a message stressing the thirst quenching properties of the drink than a text stressing its energy-boosting effects (Strahan, Spencer, \& Zanna, 2002). Similarly, participants in a sad emotional state were more readily inclined to endorse tax increases to combat saddening problems (e.g., the plight of special-needs infants) than to reduce angering problems (eg., traffic delays), whereas no such difference was found for people in a neutral state (DeSteno, Petty, Rucker, Wegener, \& Braverman, 2004). In addition, the latter study found that these effects were mediated by emotion-congruent increases in perceived likelihood (but not evaluation) that the counter-measures proposed would actually contribute to solving the problem.

\section{The Present Study}

The studies cited above, however, commonly use different persuasive messages (ie., they apply a match-mismatch design) and are therefore unable to exclude the possibility that the effects should (at least partly) be attributed to different beliefs induced by these messages, rather than to the extent that these messages match the motivational state of the receiver. Particularly, as messages are not tested for a-priori differences in their influence on attitudes 
or behavior, it remains unclear to what extent the effects should be attributed to goalmatching or to other message characteristics.

We circumvented this problem in the design of our study by investigating the effects of a single message, specifying different consequences for different goals. In other words, rather than testing the effects of different messages on similar goals, we tested the impact of a similar message on different goals. Specifically, we designed a message concerning the development of a genetically modified banana that would prevent the loss of crops to insect plagues. In the text, two primary consequences were specified should this banana be commercially produced One result would be that third-world country farmers, dependent on bananas for their livelihood and that of their families, would be saved from loosing their income. This (positive) consequence is congnuent to the goal of cooperation (i.e. helping other people). On the other hand, because of this genetic modification, bacteria that live on the banana might become resistant to antibiotics, which could cause the epidemic of a dangerous skin disease, infecting the customer of the new banana. This (negative) consequence relates to the goal of personal safety. Notably, the text stressed that extensive testing had already indicated that no other health-damaging consequences were to be expected from eating the banana. The message specified the aim of the study as investigating potential customers' (i.e, the participants') evaluations of the flavor of the banana, which was required to guarantee sufficient commercial interest to companies that had to import the banana for distribution in supermarkets.

A pretest of this message revealed that - assessed on 7-point scales ranging from 1 ("not at all") to 7 ("very much") - the text was on average considered sufficiently credible ( $M=5.5$; $S D=.97)$, convincing $(M=4.5 ; S D=1.27)$, and understandable $(M=44 ; S D=1.35)$. All means were significantly above the neutral scale mid-point. Furthermore, on a 9-point scale, ranging from 1 ("extremely negative") to 9 ("extremely positive"), people reported average attitudes ( $M$ $=4.7 ; S D=1.46$ ) towards the genetically modified banana, excluding potential bottom or ceiling effects in the experimental data. Finally, when asked to describe the major arguments in favor and against production of the genetically modified banana about one hour later, $80 \%$ of the people correctly stated both the positive consequences for farmers as well as the potential for getting a skin disease, indicating that the intended consequences are indeed sufficiently salient

In the experimental study, we investigated the consequences of activating the goals of Cooperation and Personal Safety, compared to a Control group, on the impact of the message on participants' attitudes and behavior towards the genetically modified banana. We expected induction of a Cooperation goal to positively affect people's attitudes and behavior towards the banana as the message outlined the banana to benefit poor farmers, which is conducive to the Cooperation goal. In contrast, we expected induction of a Personal Safety goal to reduce attitudes and behavior as the message specified harmful consequences pertaining to this goal. Furthermore, we assessed whether these influences would be the result of mediation by increased endorsement of the goal-related consequences or due to moderation of the impact of goal-related consequences on behavior. We expected Cooperation priming to increase the endorsement (ie., perceived likelihood ratings) of the 
benefits to the farmers or to enhance the impact of this consequence, as compared to a Control group. Similar effects were expected for the hamful consequence for personal health after priming a Personal Safety goal.

\section{Method}

Participants and Procedure. A total number of 51 students ( 42 females, 9 males, mean age $=19.2$ yrs:) at the University of Maastricht participated in this study. They came to the lab in groups of six people and received $€ 5$,-in exchange for participation.

They were welcomed by a male experimenter, who explained that they would participate in a flavor-test study. They would be handed out an information-sheet, which had to be read carefully before tasting the food product (a banana). The experimenter then asked the students if they would be willing to first participate in a nother, unrelated study of a colleague. As none of the participants refused, they were handed out the instructions and materials of this study, which was presented as a language-ability test, and was in fact the goal-priming manipulation. Participants were randomly assigned to either the Personal Safety goal-priming condition ( $(n=18)$, the Cooperation-goal priming condition $(n=17)$ or to a neutral Control group $(n=16)$. They were then seated in individual cubicles for the duration of the experiment. After completing the priming manipulation, they handed the materials back to the experimenter and received the information sheet. After reading the message described above, they were handed a plastic cup containing several slices of (an ordinary) banana (which was weighed beforehand) along with a short questionnaire. After completing the flavor test and filling out the questionnaire, they were probed for suspicion concerning the relation between both tasks and awareness of the content of the priming manipulation following a funneled debriefing procedure (cf. Bargh \& Chartrand, 2000). Finally, they were debriefed, handed their money and dismissed.

Manipulations and Measures. We used a Scrambled Sentence Test (Srull \& Wyer, 1979) in which participants constructed grammatically correct four-word sentences from randomorder sequences of five words, to prime the goals of Cooperation and Personal Safety. The test constituted of a total number of 30 sentences, 20 of which contained words or described situations implying a state of discrepancy from the goals of Personal Safety or Cooperation. Primes in the Safety condition were: riot, violent, ominous, danger, risk, phobia, frightening. terrifying, hostile, panic, war, wounded, waming, assault, murder, death, alarm, flee, and running (away). For the Cooperation priming condition, the primes were: helpless, fragile, powerless, unfair, suffer, weak, vilnerable, threatened, harassed, defenseless, deserted, poverty, need, dearth, abandoned, alone, frail, unprotected, and cast off. Sentences in the Safety condition were all stated in the first person to enhance self-relevance, whereas sentences in the Cooperation condition were all stated in third person to convey concern for another. All other words and all words in the Control condition were neutral with respect to the type of goal.

The questionnaire assessed participants' attitude by a single item asking participants to complete the sentence "I think the genetically modified banana is..." by ticking a box on a 9 - 
point semantic differential scale anchored "extremely negative" and "extremely positive" on both extremes, with a "neutral" midpoint. Subsequently, outcome expectancies were assessed by asking participants to rate their agreement to three statements on a 9-point scale ranging from 1 ("completely disagree") to 9 ("completely agree"). The statements were: "The banana will be bad for my health", specifying the personal safety related consequence. "Poor people will profit from the banana", specifying the cooperation related consequence. We also included a statement that was ostensibly unrelated to either goal, which was not explicitly mentioned in the text. This statement read: "The banana will be beneficial to the environment".

Subsequently, two control measures asked subjects to (a) indicate how hungry they were and (b) how much they felt like tasting the banana, on a 7-point rating scale, ranging from 1 ("not at all') to 7 ("very much"). Finally, subjects were asked to taste as much of the banana as needed to rate its flavor by a grade from 1 (being the lowest) to 10. After the experiment, the weight of the remaining slices in the cup was measured again, and the percentage of the total amount eaten was computed as a measure of behavior.

\section{Results}

None of the participants showed suspicion regarding a relation between both tasks, nor indicated awareness as to the contents of the priming manipulation.

Table 4.3. Mean Scores for Likelihood Ratings of Outcome Expectancies, Attitude, Flavor, and Amount of Banana Eaten by Control, Safety, and Cooperation Groups.

\begin{tabular}{|c|c|c|c|}
\hline Outcomes & Control & Safety & Cooperation \\
\hline Detrimental to Health & 3.50 & 411 & 4.71 \\
\hline Benefit to Poor People & 5.31 & 6.50 & 7.41 \\
\hline Beneficial to Environment & 5.06 & 4.06 & 5.35 \\
\hline $7 \%$ & 6.06 & 5.39 & 4.82 \\
\hline Flavor Grade & 7.20 & 6.56 & 7.06 \\
\hline Percentage Eaten & 57.9 & 428 & 56.5 \\
\hline
\end{tabular}

Between-Group Differences. An ANOVA procedure with prime condition as the sole between-subject factor was used to determine differences between experimental groups in endorsement (i.e, likelihood ratings) of outcomes, attitude, flavor ratings, and eating behavior (See Table 4.3). Results indicated reliable between-group differences in likelihood ratings on the cooperation-related outcome (i.e, beneficial consequences to poor people), $F(2$, 48) $=3.68, p=.03$. Two planned comparisons tested whether, as expected, people in the Cooperation group more strongly endorsed this outcome than people in the Control and 
Safety groups (C1:-1 -12), and whether the latter groups did not significantly differ from each other $(\mathrm{C} 2:-110)$. Results indeed showed the Cooperation group to differ from the Personal safety and Control groups, $\mathrm{C}_{1}, \mathrm{t}(48)=2.3, p=.03$, but indicated no difference between the latter groups, $C 2, t(48)=1.6, p=13$. Contrary to our expectations, however, goal priming did not significantly affect the safety-related outcome (ie, negative health-affects), $F(2,48)=160$, $p=21$. Furthemore, a marginally significant difference was found on the supposedly goalunrelated outcome that the banana would be beneficial to the environment, $F(2,48)=2.45$, $P$ $=10$. Tukey honestly significant difference multiple comparisons revealed that the Safety group considered this outcome less likely that the Cooperation group, $p=10$.

The ANOVA only showed a marginally significant difference between groups in attitude ratings, $F(2,48)=2.77, p=.07$, but not in taste ratings, $F(2,48)=67, p=.52$, or behavior, $F(2,48)=181, p=18$. Nevertheless, we also tested our expectations by a series of planned companisons, for reasons outlined in Study 3.2. Specifically we examined if Safety-primed participants would indeed have lower average scores on each of these variables than Control and Cooperation primed participants $(\mathrm{C} 1,1,-21)$, and if people in the Cooperation group showed higher average ratings than both other groups ( $C 2:-1-12)$. The only effect confirming our expectations was revealed in a marginally significant difference on $\mathrm{C2} ; \mathrm{t}(48)=$ $-190, p=06$, suggesting Safety primed people to eat less banana than people in both other groups.

Mediation Models. A hierarchical, linear, regression procedure, in which behavior, taste, and attitude were altemately regressed on the three outcome expectancies, did not yield significant models for the percentage of banana eaten by the participant, $\mathrm{R}^{2}=.06, F(3$, $46)=.94, p=.43$, nor for taste ratings, $\mathrm{R}^{2}=.05, \mathrm{~F}(3,46)=.72, p=.55$. (Adding the control variables to the models did not change the results.) Only attitudes were significantly predicted from likelihood ratings of outcome expectancies, $\mathbb{R}^{2}=.40, F(3,46)=16.33, p<.001$. As only the endorsement of the safety-related outcome reliably predicted attitude ratings, however, $\beta=-$ $.67, \mathrm{SE} \mathrm{B}=.70, t=-5.46, p<.001$, and the experimental groups did not differ with respect to endorsement of this outcome, the mediation of goal-priming by goal-congruent outcomes hypothesis could not be tested.

Moderation Models. Finally, we investigated whether goal-priming moderated the impact of goal-congruent outcome expectancies on either behavior, taste or attitude ratings. Hereto, we applied a stepwise, hierarchical, regression procedure in which each of these dependent variable was regressed on prime condition (i.e., dummy-coded variables for Safety and Cooperation) and all three outcome expectancies (Step 1), subsequently adding interaction terms of prime-dummy's and either safety or cooperation related outcomes. To cut a long story short, we did not find support for moderation effects of goal priming.

\section{Discussion}

Again, the data hinted that goal-activation might affect people's evaluations (in terms of likelihood) of goal-congruent behavioral outcomes. Specifically, activation of a Cooperation goal enhanced endorsement of the related consequence (of producing of the genetically 
modified banana) that poor people would be ensured of their livelihood Furthermore, activation of a Personal Safety goal decreased the likelihood of perceived beneficial consequences to the environment. This outcome, however, was thought unrelated to either goal Moreover, Safety priming did not affect the evaluation of the supposedly related outcome (ie, increased personal health risks).

As outcome-expectancies were introduced by a persuasive message, and concemed a topic no one was familiar with, it can be assumed that they were not strongly held In this respect the present data resembles those the previous study, suggesting the influence of activated goal states are limited to the extent that beliefs are not strongly held, which is also suggested by other authors (e.g. Schwarz \& Clore, 1996; DeSteno, et al, 2004; Petty \& Wegener, 1998).

Nevertheless, one cannot but conclude that the effects of goal-activation as demonstrated here, do not satisfactorily confirm any of the hypothesized influences of goalactivation on EV processes. Other research, however, has meanwhile provided strong support for several of the expectations conceming the potential impact of goal priming. Particularly, DeSteno and colleagues (2004) have shown that inducing emotional states by having participants read an emotion eliciting text, increases the effectiveness of persuasive messages in inducing attitude change in case the message content specifies behavioral consequences (of supporting a tax proposal) that match receivers' emotional state (sadness or anger). Furthermore, these effects were mediated by emotion-congnuent bias in likelihood estimates of these outcomes. Similar results have been obtained by studies matching message content to attitude function (Julka \& Marsh, 2000; Maio \& Olson, 1995; 2000b) and induced goal-states (Strahan et al. 2002). It thus seems premature to discard the general idea that goal-activation influences EV processes. Rather than discarding the assumptions, explanations for the indecisiveness of the present data are more likely to be accounted for by discrepancies between our study and the ones cited before, which may simultaneously elucidate several limitations in the design of the present study.

First of all, the present data suggest that - notwithstanding theoretical argumentations - practical difficulties may prevent demonstration of specific associations of outcomeexpectancies with goals. In Study 4.1, problems may have arisen because of idiosyncratic differences in the framing of behavioral outcomes. In the present study, we atternpted to exclude such influences by specifying (new) consequences in the message, which would consequently be the same for all participants in our study. Nevertheless, it seerns we may have encountered another difficulty as certain consequences, as specified in this study, may relate to more than one goal. For instance, the health-impairment outcome may be related to both the goals of Safety (when interpreted as concerning one's own health) and Cooperation (concerning the health of others). We do not take this explanation as rejecting the proposed relation between goals and specific OEs, but rather as another difficulty in sentantically capturing such associations in an unambiguous way. Future studies investigating the influence of goal-activation on outcome evaluations are therefore likely to benefit from applying self-generated outcome expectancies. 
Secondly, our study differed from others investigating matching effects of persuasive messages in that we presented multiple consequences in the same text, rather than investigating the impact of different messages specifying a single consequence. Although this excluded the possibility that the results of matching can be attributed to textcharactenistics rather than to the intended (goal) congruence, it might have also caused interference between various consequences. For instance, the (negative) healthconsequences of the genetically modified banana may have been much more conspicuous to participants than any other, which would reflect its being the sole consequence predictive of people's attitudes. Perhaps as one particular outcome appears more salient than others, this may engender relative differences in likelihood ratings that would not have occurred if each consequence were presented separately. Indeed; research has shown that the various dimensions on which OEs can be characterized do not constitute orthogonal categories and exert reciprocal influences on one another (Olson et al, 1996).

Thirdly, as we did not find sufficiently strong interelations between behavior, attitudes, and anticipated outcomes, we were unable to statistically test potential mediation of goal-activation effects by likelihood estimates of goal-congruent outcomes. To our opinion, more so than anything else, this deficiency stresses the importance of the principle of correspondence (Ajzen \& Fishbein, 1980), prescribing that different constructs (e.g. OEs, attitudes, and behavior) should be operationalized with unity of time, place, and action, in order to find associations between them. As we assessed OEs with respect to production of the genetically modified banana, attitudes towards the banana itself, and behavior by the amount of banana eaten, we obviously violated the correspondence principle, introducing a major flaw in the measures applied in the present study. On the other hand, even if a set of OEs is found to reliably predict attitudes or intentions, it might still be difficult to demonstrate whether specific OEs mediate the impact of discrete emotions/goals. A single OE would not likely be a significant predictor of people's attitude or intention, yet this would be required to statistically demonstrate mediation (Baron \& Kenny, 1986).

A fourth reason why goal-priming effects on outcome evaluations may have been attenuated is that participants may have been unmotivated to extensively process the contents of the message. If individuals are unwilling to effortful consideration of implications, goal-priming effects are unlikely to show. Several studies have indicated the impact of processing motives such as the "need for cognition" (Cacioppo \& Petty ${ }^{1982}$ ) on the effectiveness of persuasive messages. Indeed, the research of DeSteno, et al (2004; Study 2) also indicated that, if personal relevance was low, matching effects only occurred for individuals who are highly motivated to elaborate on the message content. Notably, the specified consequences of producing the banana were rather remotely connected to the goals of Safety and Cooperation, That is, complex arguments were required to outline how production of the banana affected either of these goals, which may have reduced the relevance of the message.

Fifth, influences of goal priming may have been weakened if the priming manipulation was ineffective in inducing goal-states. Most studies that prime goals by 
applying a sentence unscrambling procedure use goal-synonyms as stimuli (see: Bargh \& Chartrand, 2000, for an overview). Based on theoretical considerations (see Appendix), however, we slightly adapted the standard procedure and used words signaling goaldiscrepancy, rather than congruence. We tested the effectiveness of our priming manipulation in a series of studies, presented in the Appendix. The results of these experiments indeed seem to indicate certain difficulties in semantically priming goals by using goal-discrepant stimuli so possibly, applying a regular goal priming procedure or an emotion-induction task would have yielded stronger effects.

Finally, we wish to stress that results again indicated that goal-activation could influence behavior independent of EV processes; as was also found in the previous study. Specifically, activating a Personal Safety goal reduced the amount of banana eaten, compared to the Control group.

\section{GENERAI Discussion}

In the present chapter, we proposed an outline for an integrated perspective, combining EV and motivational (ie., goal-based) accounts of behavior. The link connecting both perspectives followed from an investigation of the motivational nature of outcome expectancies.

Various authors have stressed that predicting behavior from expectancies is motivationally vacuous (e.g., Bagozzi, 1992; Balleine \& Dickinson, 1998; Frijda et al., 2000; Hanoch, 2002; Pervin, 1989). Basically, as argued by Atkinson (1982, p. 19): "An expectation no more implies an action than does the streak of lightning imply the bang of thunder. The logical bridge between antecedent and consequent must beconstrued."

This logical bridge lies in the notion that an outcome expectancy specifies how performing an act will result in the attainment or frustration of a general goal of behavior. Hence, the motivational nature of anticipating behavioral outcomes resides in their potential to activate the same general goal systems as actually perceived stimuli do (e.g. Balleine \& Dickinson, 1992; Behrendt, 2004; Damasio, 1994; 2000; Ben-Ze'ev, 2000; Hanoch, 2002; Panksepp, 2000). In support of this idea, it was argued that the formation of outcome representations is essentially a motivated process and outcome expectancies thus develop in relation to the general goals underlying behavior (Derryberry \& Reed, 1994; Frijda \& Mesquita, 2000): Consequently an outcome expectancy can be defined as an internal representation that specifies how the perfomance of a particular act is conducive to, or frustrates the attainment of a certain goal.

Based on this association between outcome expectancies and goals, which constitutes the basic tenet of the integrated perspective, we derived several hypotheses conceming the potential impact of goal-activation on the relation between outcome expectancies and behavior. Basically, we expected that goal-activation could moderate the impact of specific, goal-related outcome expectancies on behavior. Altematively, goal-activation may also influence the accessibility of goal-relevant outcomes, or affect people's evaluation thereof, 
either in terms of likelihood or valence. We conducted two experimental studies in which we tested these hypotheses and tried to indicate possible implications of the goal-based nature of outcome expectancies for health education practice.

In general, the results of these studies were indecisive with respect to the majority of the hypotheses as just described. In some instances, this may be accounted for by limitations in measurement (e.g. violation of the correspondence principle), manipulation (eg, ineffective goal-priming), and design ( $\mathrm{eg}_{i}$ failure to incorporate control measures for people's motivation to elaborate on message content) of the particular study. Nevertheless, a more fundamental explanation may account for the lack of support provided by the present data.

A general underlying problem may reside in the phrasing of items assessing outcome representations in a way that unequivocally relates them to a specific goal Ambiguities therein may arise from idiosyncratic differences in the framing of the same outcome, which can introduce noise in individual evaluations of expectancies. Additionally, outcome expectancies may be specified too broadly, which dilutes specific associations with a certain goal. It should be duly noted that language-based approximations bear no necessary relation with the way internal representations are stored in memory (Dijker, 2001; Eagly \& Chaiken, 1993), which may complicate the identification of clear associations between anticipated outcomes and goals. The use of self-generated outcome expectancies may be the best way to overcome such problems.

Although we may not have been able to provide clear support for any of the presumed effects, we believe some tentative suggestions can be denived from the results of these studies:

1. From the results of Studies 4.1 and 4.2 , goal-activation appears to enhance the evaluation of goal-related outcomes, mainly in terms of likelihood (see also: Desteno, et al., 2004), and especially in case these outcomes are not highly accessible to individuals. These findings are in line with connectionist models (e.g. Anderson, 1983), in which the salience of OEs (and other cognitive constructs) is viewed as dependent upon situational triggers (i.e. emotions). Rather than as stable representations, connectionist models picture attitudes or intentions as temporary, composite states of temporary activated beliefs. Consequently, different belief-sets may underlie intentions on varying occasions (Lowe et al, 2003).

2. From the results of Study 4.2 and similar research in the domain of attitude functions (Iullka \& Marsh, 2000; Maio \& Olson, 2000c) and persuasion (DeSteno et al, 2004; Strahan et al., 2002), it seems that goal-states, whether dispositionally or situationally activated, may provide a fruitful target for matching messages in the domain of health education. That is, matching message content to chronical or situational variation in goal-activity may enhance the impact of messages aimed at attitude or behavior change. As such, goals provide another means to tailor health-education.

3. Finally, both Studies 4.1 and 4.2 indicate that goal-activation can influence behavior independent from reasoned-action processes. Consequently, it may be difficult to capture the full range of motivational influences on goal-directed behavior in EV accounts. 
In conclusion, we would dissuade from discarding the basic idea that goal-activation, particular by induced emotional states, may influence the processes generating behavior as described in EV models. We feel confident in advocating this position, not only because of the promising nature of the present results, but also because the consequences of this idea suggest new approaches to health-education attempts at behavior change. This latter notion will be elaborated in the final chapter, in which we will also explore the potential boundaries the integrated perspective imposes on information-based health-education. 


\title{
Chapter 5
}

\author{
"Forbidden Fruit"
}

\section{Reflections on the Consequences of the Integrated Perspective}

\section{INTRODUCTION}

In the previous chapter, we integrated reasoned action and motivational (ie., goal-based) accounts of behavior by associating action-specific outcome expectancies to the general goals underlying human behavior. Hereto, an $\mathrm{OE}$ was defined as an internal representation that specifies how performing a particular act is conducive to or frustrates the attainment of a general goal The emanating view was refenred to as the integrated perspective.

\section{A Summary of the Integrated Perspective on Behavior}

The integrated perspective holds that, because of their association with the general goals underlying behavior, the impact of OEs on behavior is (at least to some extent) dependent upon the operation of the basic motivational systems, directing action aimed to attain these goals. The integrated perspective implies two ways in which reasoned action, that is, deliberated; volitional behavior, follows from a particular choice-situation, both of which can be involved in the generation of a single behavioral response (see Figure 4.1).

On the one hand - as described in current EV models - when deliberating a behavioral decision, anticipating certain consequences of acting implies the attainment or frustration of 
certain goals and thereby provides a motivational drive for a certain course of action. For instance, I may believe that a rejection of the European constitution will impair the successful prevention of terronist attacks, which would conflict with my goal to be safe. Activation of this safety goal, possibly accompanied by feelings of anxiety, would incline me to vote favorably in the upcoming election. In accordance with this process, behavioral differences arise from individual differences in the extent to which people endorse particular outcome expectancies with motivational potential. Motivational potential refers to the amount of goal activation caused by perceiving the performance of an act to have a certain consequence: In contrast to the previous example, I may also believe that ratification of the constitution will enable more uniform guidelines conceming the number of potatoes produced for export. Yet this does not conoern me and therefore to me, it is not relevant to any goal: In other words, this outcome has no motivational potential for me.

On the other hand - and not implied by current EV models - the activation of a behavioral goal in a decision situation may affect people's perceptions of certain outcomes as well as vice versa. This, in tum, may influence the relation between certain OEs and behavior. For instance, if I want to help other people, I may be inclined to believe that underprivileged people will benefit from a ratification of the European constitution, which makes this likely to be an important determinant of my voting decision. Behavioral decisions may thus also ensue from individual variation in the extent to which certain goals are activated in the decision situation. In this way, deliberative, goal-directed behavior is based on anticipatory images in accordance with the overall motivational state of the organism. Notably, goalactivation can be the result of both situation-relevant (ie, integral) and non-relevant (i.e, incidental) motivational cues. In the latter case, for example, when making my decision to vote, I might still be angry from an argument I had earlier, invoking an action tendency to get back at someone. This could cause me to believe that if the constitution is approved, foreign people will come to take advantage of the Dutch social security system, inclining me to vote against ratification.

\section{Summary of Research Findings Concerning the Integrated Perspective}

In our studies, we investigated this influence of goal-activation on reasoned action processes. Several hypotheses were derived conceming the influence of goal-activation on the relation between OEs and behavior. Firstly, goals may affect the accessibility or evaluations (in terms of likelihood and/or valence) of anticipated outcomes. Secondly, goal-activation may affect (i.e., moderate) the influence of OEs on behavior.

Neither our own studies, nor others' provide support for the moderating influence of goal-activation on OE - Behavior relations. We did not find support for the influence of goalactivation on the accessibility of related OEs either. The results of our studies did, however, suggest an influence of goal-activation on people's evaluation of goal-related OEs. In Study 41, goal-activation (by induction of emotional states: fear and guilt) was found to enhance expectancy-value ratings of anticipated outcomes of intervening with random violence that were associated with the goals of fear ("If I intervene I run the risk of becoming a victim 
myself") or guilt ("If I intervene I will be able to help the victim"), respectively. These effects of goal-activation, however, were limited to the extent that the $O E$ was not strongly associated (as indicated by slower response latencies) with the behavior. Quite similarly, in Study 42, we found that activating the general goal of Cooperation increased people's endorsement (ie, perceived likelihood) of consequences (of producing a genetically modified banana) that were relevant to this goal (ie, that it would benefit poor people). Again, this effect only concerned weakly associated outcomes, as the consequences of producing the banana were specified in a message that was fictional and thus novel to all participants. Notably, a similar pattem emerged in Study 3.2, in which we demonstrated that temporanily induced emotional states affected behavioral decisions (in a prisoner-dilemma game), only if people did not already have a chronically active situation-relevant behaviaral goal evoking similar behavioral tendencies as the induced emotional state. Together, these results thus seem to provide preliminary support for an influence of goal-activation on people's likelihood perceptions of goal-relevant behavioral outcomes that they not immediately associate with performing the behavior under investigation.

Finally, it should also be noted that the results of Studies 41 and 4.2 indicated that goalactivation could also directly affect behavior. The results of Study 4.1 suggested that an induced emotional state (fear) reduces intentions towards behavior (interfening with random violence) that would be in conflict with the general goal of fear (enhancing personal safety). Similarly, the results of Study 4.2 indicated that inducing a personal safety goal by semantic priming procedures reduces behavior (eating a genetically modified banana) to which negative implications for personal safety had been attributed by a message. Notably, this influence was not mediated by expectancy-value estimates of relevant OEs. Moreover, in both instances, participants were unaware of the priming manipulation by which the goal was activated.

Perhaps the most elegant summary of the integrated perspective is provided by David Hume in his Treatise of Human Nature (Hume, 1739-40/1978, p. 415, as cited in: Colman, 2003):

Reason alone can never be a motive to any action of the will, and ... can never oppose passion in the direction of the will... Reason is, and ought only to be the slave of the passions, and can never pretend to any other office than to serve and obey them

Indeed, reason cannot be a motive, as the anticipation of outcome expectancies will only cause behavior if the outcomes are capable of activating the general goals underlying one's action in a given situation. Furthermore, reason is the slave of the passions as the impact of OEs on behavior is affected by the activation of goals (ie., by the ellicitation of emotions). Finally, as will be outlined in this chapter, intentions, based on the deliberation of 
consequences, are often overidden by emotional responses instigated by environmental. cues, hence; reason cannot oppose passion in the direction of the will

\section{Overview of the Present Chapter}

In this final chapter we will address several consequences of the integrated view. In the following paragraph we will first consider the contributions of relating general goals to current EV models. In addition, we will evaluate whether the (four) reasons, forwarded by Ajzen and Fishbein (1980) to omit distal variables from the TPB, as addressed at the end of Chapter 2, would also apply for the inclusion of general goals in the face of the arguments and findings favoring the integrated perspective. Subsequently, we will outline implications for behavior change. The reasoned action-perspective, as described in Chapter 1, holds that behavior change can be effectuated by the communication of information conceming potential consequences of actions, for instance by persuasive messages in the field of health education In the previous chapter we already suggested that the integrated perspective might extend the basis for information-based methods to induce behavior change, by identifying individual or situational differences in goal activation as a potential target for health intervention messages. This suggestion will be elaborated in the present chapter. We will also, however, concentrate on implications of the integrated perspective that may confine the scope and limit the impact of information-based health education. These implications are not directly apparent from EV models and may contribute to our understanding of the effectiveness of information-based health education methods. We will then suggest health-education efforts should also focus on other means of behavior change that follow from the integrated perspective on behavioral control Specifically, given the profound influence of situationally induced emotions on behavioral decisions, we will advocate that increased attention should be paid to emotion-regulation processes.

Throughout the discussion in this final chapter, we will sidestep the limitations to our studies as discussed in Chapter 4. Perhaps, some of the statements and conjectures to be presented may therefore seem a bit bold in the face of the limited empinical support for the integrated perspective as demonstrated in previous studies. Nevertheless, to our opinion, a consideration of these consequences elucidates the potential advantages of the integrated perspective as it suggests new avenues for future research in the fields of health education and health psychology.

\section{CONSEQUENCES FOR EXPECTANCY-VALUE MODELS}

In this paragraph we will first outline how our understanding of the behavioral processes described in EV models may improve from incorporating general goal constructs. We will then argue that the integrated perspective contradicts most of the arguments forwarded by Ajzen and Fishbein against the inclusion of distal variables into their model. 


\section{Contributions from Incorporating Goals}

It should be noted explicitly that the integrated perspective by no means invalidates reasoned-action accounts of behavior as formalized in EV models. On the contrary, it substantiates them, first of all by specifying the motivational nature of anticipated outcomes, which is not apparent from cument EV models. By exploring how the formation and influence of $O E s$ depends upon activation of the basic motivational systems directing action to the general goals underlying behavior, perceived consequences have, once more, been causally connected to action.

The qualification "once more" is used deliberately, for the link between OEs and motivation was specifically addressed in several original EV conceptions of behavior. The following description, for instance, comes from Atkinson's EV model of achievement motivation (Atkinson, 1980, p. 25):

The term, expectancy ... refer[s] to a particular kind of cognitive association aroused in the person by situational cues.... We are interested in expectancies, which signify consequences, which are incentives ... of some motive.... The subjective value or utility of that incentive for a particular individual depends upon the strength of his motive.

Similarly, a link between people's attitude towards an object and the potential of that object to attain or block personally important values (ie., goals) was the basis of Rosenberg's (e.g., 1956) research on the cognitive structure of attitudes. Specifically, Rosenberg showed that people's attitudes could be predicted from the sum of products derived from their ratings of (a) the importance of a set of general values and (b) their perceptions of the extent to which interaction with the attitude object would lead one to achieve or block these values. Hence, in this model, general goals rather than behavior-specific OEs were used to predict attitudes. Later, general EV models of behavior, like the TPB (Ajzen, 1991), abandoned the goal-based onigin of OEs for the sake of predictive accuracy. The link with goals is now re-established in the integrated perspective.

Besides explicating the causal influence of OEs, the integrated perspective may elucidate some findings that appear to conflict with assumptions underlying the TPB. For instance, the finding that some beliefs are more important determinants of attitudes and behavior than others (van der Pligt, de Vries, Manstead, \& van Harreveld, 2000) violates the notion that all beliefs should have equal weights (Sutton, 2002a). One of the hypotheses derived from the integrated perspective - though not supported by results from the present studies - predicts, however, that goal activation may directly influence (ie., moderate) the relation between OEs and behavior for goal-congruent beliefs. For instaince, if I am hungry, the anticipated satisfaction from consuming a Big Mac is likely to outweigh the importance of it being bad for my health, whereas the latter may seem more important if I just had dinner. Hence, motivational states may determine the relevance of perceived consequences for making a behavioral decision. 
A final contribution of specifying the goal-based nature of OEs is that it allows for a more general understanding of behavioral determinants than is provided by current EV models. The TPB in particular has been criticized for sacrificing insight into the ultimate determinants of broad behavioral patterns, to predictive accuracy of specific actions. This is mainly a consequence from adherence to the correspondence principle in measuring the model variables (Eagly \& Chaiken, 1993; Elliot \& Thrash, 2002; Swanbom, 1996; Weinstein, 1993). On the other hand, the assessment of general goal constructs is unlikely to yield the level of predictive accuracy in terms of the percentage of variance as provided by EV models. As argued in Chapter 3, the equifinality principle (e.g. Kruglanski, 1996) holds that general goals can be attained in multiple ways. Hence, strong relations between a general goal and a specific act are not to be expected. Furthermore, concepts framed in terms that people actually use in thinking about their own behavior, like EV variables, will undoubtedly lead to better predictions than concepts stated in more general terms, like goals, values, or emotions (Weinstein, 1993). Nevertheless, by linking general goals to behavior-specific OEs, the integrated perspective provides EV models with a more fundamental layer in the understanding of behavion. Thus, the integrated perspective combines the strengths of reasoned action models (i.e., predictive accuracy) and motivational accounts (i.e, insights into general determinants) of behavior.

\section{What about Social Influences and Perceptions of Control?}

Throughout the dissertation, we have been mainly concemed with the association between general goals and behavioral outcome expectancies. Relations between goal activation and social influences (e.g, subjective norms) or control beliefs (e.g., as underlying perceived behavioral control and self-efficacy), have not explicitly been addressed, nor empirically tested Nevertheless, a few speculative remarks may illustrate how control constructs and social influences may derive from general goals as well.

To start with the latter, the social norm construct of the TPB is determined by an individual's perception of what other, personally important people would like him to do (i.e, normative beliefs); and his or her motivation to comply with these important others. One can easily envisage a motivation to comply arising from several social emotions, like empathy, love, pity, guilt, and gratitude, engendering behavioral tendencies to conform to the wishes of other people. For instance, the guiltier I would feel for my partner's bereavement after my dying from the consequences of smoking, the more I would be motivated to comply to her pleas for me to stop smoking. Not surprisingly, many young parents quit smoking during pregnancy of their firstchild.

A link between perceptions of control or self-efficacy over performing a specific action, and the general goals as identified by human ernotional experiences, may follow from two ways in which emotions can affect people's experience of control in a given situation. First of all, research demonstrated emotional states to be associated with certain appraisal tendencies (e.g, Lerner \& Keltner, 2000), which refer to tendencies to perceive a situation in accordance with the basic appraisal dimensions characterizing the emotion (Smith \& 
Ellsworth, 1985). Some emotions (eg, anger, and happiness) are associated with the experience of high levels of personall control over the outcome of a particular situation. Others (e.g, fear) are characterized by a sense of situational (i.e, low personal) control. It seems plausible that the former translate into high levels of self-efficacy or perceived control over behavioral outcomes, whereas the latter result in low selfefficacy or perceived control.

Secondly, the experience of certain emotions (eg, pride and shame), which are referred to as self-conscious emotions (cf. Lewis, 2000), is associated with fluctuations in self-esteem (Brown, Dutton, \& Cook, 2001), defined as a person's overall positive or negative selfevaluation. Measures of self-esteem are usually strongly conrelated with measures of control, like self-efficacy and locus-of-control (Judge; Erez, Bono, \& Thoresen, 2002; Stanley \& Murphy, 1997). Hence, the experience of self-conscious emotions may directly affect an individual's perceived control over performing an action.

\section{Should We Expand EV Models with Goal Constructs?}

In spite of the theoretical contributions from integrating EV models with general goal constructs, Ajzen and Fishbein (1980) mentioned four reasons to abstain from incorporating distal variables into their model First of all, they argued that distal variables would affect behavior only through the variables specified in their model Nevertheless, both Studies 4.1 and 4.2 indicated independent effects of goal-activation on behavior (-al intentions), which illustrates that other than reasoned action processes might underlie volitional behavior.

Secondly, it was argued that distal variables would not contribute to the TPB, as consistent predictions conceming the way such vanables influence behavior or any of the intermediate variables cannot be made This claim was mainly based on inconsistencies in associations found between behavior and factors like personality characteristics, socioeconomic status, and age, for example. Nevertheless, the argument does not seem to apply to general behavioral goals, as the implications of a particular action for one or more of these goals seems readily predictable. Behaviors as diverse as organ donation, condom use, interfering with random violence, and eating genetically modified food, seem to bear consistent implications for general goals like "helping others" or "avoiding personal harm". Furthermore, the assumptions underlying the integrated perspective, which were at least partly supported by our studies, indicate that - notwithstanding certain intricacies related to their framing - even specific OEs can be related to these general goals.

The third reason for not incorporating other variables into the TPB was the expectation that different variables would be required for different types of behavior, compromising the generalizability of the model Nevertheless, as emotions appear to be universal psychological phenomena, and neuro-anatomical criteria provide an objective criterion for deciding on the nature of basic emotions, surely, a set of goals derived from emotions will still allow for a general model of behavior. Although a final set of fundamental goals awaits future evidence, the apparent possibility of its composition contradicts the claim that adding constructs would violate the generalizability of the TPB. Note that this does not imply that each goal is involved in every behavioral decision, yet neither are all constructs from the TPB. 
Finally, Ajzen and Fishbein contented that the validity of the TPB does not demand the absence of distal influences, but merelly the demonstration of associations between variables as specified in their model As introduced in Chapter 1, and outlined in Chapter 2, however, more is required to ensure the psychological validity of EV models in general and the TPB in particular. Specifically, for EV models to provide a psychologically valid account of behavioral causation by ariticipation of outcomes, behavioral expectations had to be related to general goals. The integrated perspective provides this link. So, we subscribe to Ajzen and Fishbein's position that the model's validity does not require the absence of distal variables (i.e, goals), but go a step further by arguing that it does require their presence. Illustrating the consequences of the difference between these statements, in the subsequent paragraph we will discuss how the associations between goals, OEs, and behavior as stipulated in the integrated perspective, has implications for information-based health education that could not have been recognized from current $E V$ models.

For now, we will not prescribe how the TPB or other EV models should be expanded with general goal constructs. First of all, a final set of goals is not presently available; although we think that neuropsychological research into the brain substrates of discrete emotions will eventually settle this matter. Secondly, the present data do not yet allow unambiguous representation of the way in which goal-activation affects reasoned action processes. Future studies should therefore explore the hypothesized relations schematically represented in Figure 41 , to determine which of the proposed associations accurately represent these influences and which are redumdant. Nevertheless, we feel confident in stating that EV models eventually are to be expanded with general goal constructs.

\section{CONSEQUENCES FOR BEHAVIOR CHANGE BY INFORMATION- BASED HEALTH EDUCATION}

In Chapter 1, we introduced persuasion as the primary means of health-education to ensue from the reasoned-action perspective on behavior change. Specifically, as EV models stress the importance of people's considerations as determinants for action, persuasion aims to change existing perceptions concerning the consequences of action or to specify additional consequences, in order to induce the performance of desired behavior (O'Keefe, 1990; Sutton, 2002b). We have referred to this approach as "information-based" health education. Notwithstanding various other means by which health education programs may achieve sucoessful behavior-change, the transfer of information immediately follows from the reasoned action perspective as formalized in EV models.

In this paragraph, we will argue that the integrated perspective introduces several implications for information-based health education that are not apparent from current EV models. Specifically, we will argue that the effectiveness of information-based health education is restricted by the integrated perspective in three ways: Firstly, because the impact 
of $O E$ s on behavior is dependent upon an individual's motivational state, which is liable to situational fluctuations. Consequently, even if the receiver accepts the consequences as specified in a persuasive message, they need not exert a constant impact on behavior. Secondly, because several factors cause the OEs communicated by persuasive messages to be held with lesser certainty compared to experience-based beliefs. This attenuates their behavioral impact, or in other words, their motivational potential Nevertheless, we will also address several ways in which the motivational potential of persuasive messages may be improved by acknowledging implications of the integrated perspective. And thirdly, because goal-activation may affect behavior directly, independent from reasoned action processes. In such instances, direct motivational influences predominate over premeditated intentions in the control of behavior. Each of these restrictions will be discussed separately in the subsequent sections.

\section{Motivational fluctuations}

To be effective, persuasive communication aims to induce enduring changes in an individual's belief-set (O'Keefe, 1990). Hence, newly introduced or altered outcome expectancies have to be capable of sustaining activity over long periods of time, and under variable circumstances. It is exactly this temporal stability requirement that is undercut by the integrated perspective.

Several authors have stipulated that measures of variables in EV models often lack temporal stability (Elliot \& Thrash, 2002; Rhodes, Coumeya, \& Jones, 2004; Sheeran \& Abraham, 2003). Indeed, temporal (in)stability has been marked one of the principle limitations of the TPB, judging from the following statement (Ajzen, 2002, p.120): "the limits of reasoned action are ... inaccurate ... beliefs [and] weak or unstable attitudes or intentions". Note, however, that Ajzen does not explain why beliefs may be inaccurate and intentions unstable. This can be accounted for by the integrated perspective, which explains instability in terms of shifts in motivational state.

The results of Studies 4.1 and 4.2 , suggest that situational variation in motivational state (ie., the level of goal-activation) will increase people's likelihood estimations of goalrelated outcomes, at least to the extent that these outcomes are not already strongly associated to the performance of the behavior. According to current EV models, this enhances their impact on behavior. Other studies not only corroborate these findings by showing similar influences of motivation on probability estimates (DeSteno, Petty, Wegener, \& Rucker, 2000; Lerner \& Keltner, 2001), but also suggest, by mood-congnuent influences on accessibility of $\mathrm{OEs}$, the possibility that goal activation may affect the accessibility of certain anticipated consequences over others (Goldstein, Wall, McKee, \& Hinson, 2004; McKee, Wall, Hinson, Goldstein, \& Bissonnette, 2003).

Consequently, the relative impact a particular $\mathrm{OE}$ has on people's decisions to engage in action depends on the goals that are activated at the time of decision-making. Also, due to motivational fluctuations, the reasons for engagement in a certain act as provided in a neutral state (e.g., by a participant completing a questionnaire) need not coincide with the 
considerations that actually influence behavioral chice at the time of decision-making. Research on automatic goal-activation (Bargh \& Chartrand, 1999; Bargh \& Ferguson, 2000), and non-conscious activation of emotional states (LeDoux, 1996; Zajonc, 1998), suggests that environmental cues can activate motivational systems even in the absence of conscious awareness of these stmuli Consequently, motivational fluctuations are apt to occur frequently. Note that this view is in line with connectionist models (eg. Anderson, 1983); according to which the belief set underlying the momentary assessments of an intention may differ on varying occasions (eg, Lowe, Bennett, Walker, Milne, \& Bozionelos, 2003).

As a result of motivational influences on the relation between OEs and behavior, even the acceptance of information by the recipient of a persuasive message does not guarantee that the induced belief will exert a constant influence on his or hers behavioral decisions.

\section{Motivational potential}

A second way, in which the effectiveness of information-based health education is limited, relates to what can be referred to as the "indubitability" of an outcome (cf. Frijda \& Mesquita, 2000). Indubitablity refers to the extent that a behavioral outcome cannot be called into question and constitutes an important determinant of the perceived certainty of an outcome expectancy. The indubitability of an $\mathrm{OE}$ that is induced by a persuasive message is limited for two (interrelated) reasons.

The first follows from the process of belief formation. Basically, attempts to induce OEs by persuasive communication ignore the natural way in which cognitive representations are formed, that is, through interactions with the environment (ie, through direct, as opposed to indirect experience). As outlined in Chapter 4 , the outcome of such interactions are compared to the level of attainment or frustration of the goal(s) underlying the individual's behavior to generate the action-consequence link that characterizes an $\mathrm{OE}$ (Balleine \& Dickinson, 1998). The extent of goal attainment or frustration implied by the particular outcome, which is reflected in the intensity of the emotional response to the outcome, determines the indubitability of the outcome, and consequently, the certainty with which a particular OE will be held (e.g. Frijda, 1986). This ultimately affects the impact of the $O E$ on behavior. Persuasive messages, however, can be regarded as a "short-cut" to the formation of OEs. Verbal communication, essentially being more abstract, is usually not associated with the same level of emotional experience as sensory experience. Cornmunicated outcomes are thus less indubitable, and will be held with less certainty. Their motivational potential is therefore limited.

Studies have indeed shown that expectancies deniving from direct experience are held with greater certainty, and are better predictors of behavior than expectancies based on indirect experience (Fazio \& Zanna, 1981). Furthermore, direct experience appears to contribute to the generation of affective attitudes (Millar \& Millar, 1996). Additionally, affective components of attitudes are more accessible from memory and thus more likely to influence behavior (Verplanken, Hofstee, \& Janssen, 1998). Such findings are readily interpretable from the integrated perspective. 
Secondly, communicated consequences are open to doubt because they often entail future and/or probable consequences (e.g, the possibility to get cancer from smoking for several years). Pertaining to the former, studies show that the motivational impact of perceived future consequences to reduce with increasing time lag from the current moment (Kamiol \& Ross, 1996), a phenomenon also referred to as "time discounting" (Frijda \& Mesquita, 2000). Indeed, many unforeseen events can interfere between current and future affairs. Furthermore, possible consequences are not as convincing as actually perceived ones. Everyone has a chain-smoking grandfather who just turned ninety-nine last week to attest for the fact that smoking may not have to cause sickness at all, yet many smokers know with absolute certainty from personal experience that smoking can feel great. Both factors reduce the indubitability of communicated consequences, limiting their perceived certainty and thus their motivational potential to induce behavioral change.

\section{Suggestions from the integrated perspective to boost the efficacy of persuasive messages}

In the previous chapter we suggested that - notwithstanding these restrictions - the integrated perspective also suggests that relevant goals may present a basis for the tailoring of health-education programs (de Vries \& Brug, 1999). In general, it can be expected that the motivational potential of communicated consequences will be higher if they correspond to presently activated goals. We suggest four ways in which the motivational potential of persuasive messages can be improved from considering goal-activity.

First, the content of a persuasive message can be tailored to the motivational profile of the person, that is, to dispositional, individual differences in activation thresholds of general goals. When advocating regular exercise, for instance, a person who attributes relatively greater impartance to his personal well-being, will be more susceptible to arguments concerning his health, as opposed to a person who's main concern is his physical appearance. The latter will be more readily persuaded by appeals to his looks. As demonstrated in Study 3.1, individual differences in chronic activity of general goals can be assessed by ratings of value importance or by frequency estimates of the experience of emotional states.

Secondly, the content of a message can also be tailored to the motivational profile of the behavior under investigation. The goals that are likely to underlie a particular action can be determined by asking respondents to rate the extent to which the act is conducive to, or frustrates the attainment of general goals (cf. Rosenberg, 1956, see also: Study 31), or by assessing anticipated emotional responses to (not) performing the behavior. If for instance, a respondent indicates that not separating his garbage would make him feel guilty, a persuasive message could stipulate his duties to society, appealing to his concerns for other people.

Thirdly, when trying to change exosting beliefs, persuasive messages should target outcome expectancies with the greatest motivational potential. Although we did not investigate this in our studies, the integrated perspective suggests that OEs with the greatest motivational potential are the ones associated with the most intense anticipated emotions. Starting from a set of individually or modally salient beliefs, respondents could be asked to 
rate the intensity of goal-related emotional reaction to each outcome. If, for instance, a respondent indicates a particular outcome (e.g." "If I would have sex without using a condom I run the risk of getting HIV") would only evoke a modest emotional response (in this case, fear), the outcome is not likely to be motivationally salient. As such, health-educators may obtain a clearer picture of the relevant OEs than can be derived from a list of salient beliefs. Furthemore, the motivational potential of to-be-induced beliefs can also be pre-tested this way.

Finally, persuasive messages can also be tailored to previously induced motivational states. In Study 4.2 we found that activating the goal of Cooperation incurred greater endorsement (ie., perceived likelihood) of goal-congruent outcomes as specified in a message (ie, that the banana would benefit poor people). Similarly, DeSteno at al. (2004) found persuasive messages matching receivers' emotional state to be more effective in engendering support for a tax proposal. Hence, persuasive messages will be more effective if preceded by a strong motivational cue, such as visual images (e.g. of helpless, bereaved people). Note that a particular goal-state need not necessarily have been induced by cues that are relevant to the concem raised by a persuasive message. Goal activation may also exert an influence on unrelated decisions. In this respect it may be worthwhile to investigate if requests, for instance for people to register as organ donors, are more effective after an incident that likely induced a positive mood state (which generally causes people to behave more cooperatively), like the national soccer team winning an important match.

Additionally, other means of health education may be more likely to induce more indubitable outcome expectancies than information-based methods. For instance, vicarious learning and modeling techniques (e.g., Bandura, 1986), by which people leam (about the consequences of behavior from observing others, may provide a more credible source of information than a simple message. Finally, the use of anticipated emotions (Richard, de Vries, \& van der Pligt, 1998), by asking people to imagine how they would feel if they would perform a particular behavior, may also induce the formation of OEs with motivational potential, as affective responses are invoked just as during the actual experience of an outcome.

\section{Motivational dominance}

Apart from attenuation of the potential influence, the integrated perspective also implies a restriction of the scope of information-based health education, by indicating a direct impact of goal activation on behavior. The existence of direct (ie, non-deliberated) action control can restrict the scope of behavioral interventions based on persuasive communication that aim to affect EV processes.

Underscoring such direct motivational influences, Loewenstein and colleagues (Loewenstein, Weber, Hsee, \& Welch, 2001) differentiate between anticipatory and anticipated emotions. The latter, as discussed in Chapter 2, refer to affective consequences expected to result from a behavioral decision, and as such may be conceived of as a (distinct) 
kind of $\mathrm{OE}$. Anticipatory emotions, on the other hand, are immediate reactions experienced at the time of decision-making. For instance, a sudden flash of panic when witnessing an act of random violence, which causes one to run away, in spite of one's intentions to always come to a victim's aid. Contrary to anticipated affect, they cannot be taken into account by EV models as their elicitation is not the result of premeditating behavioral consequences, but of situational cues. Thus, anticipatory emotions influence behavior independent from reasoned action processes.

Anticipatory emotions may diverge from behavioral decisions based on cogritive anticipations, resulting in behavioral conflict. For instance, most smokers know that cigarettes are unhealthy, yet still keep smoking because it feels good (or bad not to). A discrepancy between deliberated intentions and momentary emotional influences may occur because these emotions arise in response to situational variables that do not enter into cognitive evaluations (Loewenstein et al, 2001). For instance, when deliberating a future course of action people may picture the decision situation less vividly (i.e. more abstract), neglecting cues that elicit emotional responses that conflict with their intentions. Additionally, the discrepancy may be the result of previous, irrelevant emotional experiences, carrying over to the present situation (Schwarz \& Clore, 1996).

Notably, when such a divergence occurs, more often than not, the situationally induced emotional response exerts a dominant influence on behavior over that of the deliberated course of action (Gifford, 2002; Loewenstein et al, 2001).

\section{Neuro-Evolutionary explanations for emotional dominance}

This observation can be accounted for by the neuro-anatomical structure of brain regions mediating intentional and emotional control of behavior. Specifically, the latter (ie., the amygdala and thalamic centers) are reciprocally connected to the brain centers underlying volitional control of action (ie, the dorsolateral prefrontal cortex and the anterior cingulate cortex). Nevertheless, neuronal trajectories projecting from the emotional regions to the higher cortical centers, greatly outnumber similar pathways returning from the volitional regions (Barbas, 1995; LeDoux, 1996). As such, the connections between the brain regions mediating emotional and volitional control substantiate the observed dominance of situationally elicited emotional impulses over premeditated intentional ones.

Moreover, the predominance of "emotional" brain regions in the control of behavior is readily understandable from an evolutionary point of view (MacLean, 1990, see also: LeDoux, 1996; Ploog, 1989; Plutchik, 1991). The evolutionary development of emotional brain structures substantially preceded the development of the higher cortical regions. Hence, the adaptive value of the psychological mechanisms mediated by this part of our brain was already well established after millennia of natural selection, when cortical functions still constituted an evolutionary quirk. Tuming over control would be the adaptive equivalent of the Cultural Revolution in communist China, during which period children ran the major institutions. 
Still, impulsive, emotionally induced actions, arguably, comprise the bullk of our health-impaining behavior. They cause us to succumb to our desire for fat and sugar-rich food products, and our craving for nicotine, alcohol, and other addictive substances. Also, they evoke tendencies for aggressive outburst and other antisocial conduct Such detrimental consequences of emotional behavior are assumed to anise as a byproduct of cultural evolution outpacing biological evolution (Cosmides \& Tooby, 1987). As outlined in Chapter 3, emotions instigate situation-dependent response tendencies that have proven valuable for long periods during evolutionary development. However, responses that served our ancestors well are not always appropriate in modem societies. Especially technological developments have thrown emotion-instigated action off the adaptive track. The "freezing" caused by sudden fear or startle makes us hit the brakes when driving over $120 \mathrm{~km} / \mathrm{h}$ on the freeway with many other cars driving behind us; in stead of punching someone out of anger, people now get shot; whereas sugar or fat-rich food products were once hard to obtain thus worth the satisfaction, they can now be obtained on every street comer; and joy derived from difficult achievements is now available in syringes.

Just as evolved emotional response tendencies may preserve unhealthy behavior, they may complicate the adoption of healthy conduct. This impediment lies in the fact that many healthy actions only become rewarding in the long run, but are often negatively reinforced at the time of execution. For instance, exercising several times a week is time-consuming, tiring, sometimes even boring, and often expensive, yet will only improve health if maintained for a sufficiently long period of time in spite of these immediate costs. Of course, health educators have developed techniques to incur people to persist in such behaviors. For instance, by giving feedback on one's progress (e.g., by signaling improvement in physical endurance or reduction in body weight), or by having people reward themselves (e.g, by buying something nice) after a few weeks of maintaining the desired behavior (e.g., Bandura, 1986; Brug, et al, 2000).

Both these strategies can be considered means for people to regulate their emotional experience. Emotion regulation can be an important tool for health education, especially to limit affective influences that cause people to abstain from healthy behavior as described in this section. We will discuss emotion-regulation processes in greater detail in the next paragraph

\section{Summary}

To recapitulate, according to the integrated perspective, the content of OEs is linked to the general goals underlying behavior, and their impact on intentions and behavior is therefore (at least to some extent) related to the operation of the basic motivational systems (i.e., emotions) directing action aimed to attain these goals. The consequences of the integrated perspective on behavioral control may imply certain limitations for the effectiveness of behavioral interventions applying information-based methods because: 
1. The influence of $O E s$ on behavior is not constant but dependent upon fluctuations in the motivational state (i.e. activated goals) of the individual.

2. Communicated outcomes often lack motivational potential (ie, the capacity to influence behavioral decisions) because they are generally held with less certainty than OEs that are based on direct experience.

3. Goal-activation may influence behavioral decisions independent from and predominant over reasoned action processes. Though not in contrast, these implications are not directly apparent from EV models of behavior, which may have caused an overestimation of the potential to induce behavior change by means of information based health education.

\section{Further Consequences for EfFective Behavior Change}

Given their profound influence on health related behavior, and their central role in the integrated perspective on behavior; we argue that the understanding of emotional processes warrants great attention from researchers in the field of health education. Specifically, insight in the ways in which people try to regulate their emotional experience may prove of great value to develop effective means of behavior change. Emotion regulation processes are the central focus of this paragraph

\section{Emotion regulation}

The integrated perspective stresses the importance of reactive (i.e., non-deliberative), emotional influences in action control. Nevertheless, behavioral tendencies resulting from momentary emotional experience may be in conflict with and override premeditated plans (Loewenstein et al, 2001). These emotional impulses are elicited by situationall cues and direct behavior in response to immediate concems at the expense of negative long-term consequences. This is the case when we let our momentary desires and impulses prevail over our plans to eat healthier, practice safe sex, or to not respond to provocation from the people mocking us in the pub. These examples illustrate the importance of research on emotionregulation:

Emotion regulation is defined as the processes by which people attempt to influence what emotions they experience, when they experience them, and how they express them (Gross, 1998). Note that emotion-regulation not only entaills the averting of unwanted feelings but also attempts to evoke pleasant ones. Based on the sequence of events underlying the generation of an emotion induced behavioral response; as outlined in Chapter 3, Gross specifies five sets of emotion regulatory processes that impact at different points in this chain.

Specific strategies, methods, and techniques are already available to health educators to enhance emotion control in relation to each set of processes, as will be described in the following. Though new tools may be developed in the future, the immediate advantage of 
this survey of emotion regulation processes resides in providing a comprehensive framework for the development of interventions aiming to change behavior that appears susceptible to reactive emotional influences. Each type of emotion-regulation processes will be outlined briefly:

First, situation selection refers to decisions to approach or avoid certain people or objects in oxder to influence particular emotional experiences. For instance, looking for a new place to live in order to escape from the noisy upstairs neighbor throwing daily parties till 3:00 am Pertaining to health-related actions, people may adopt a variety of strategies to select situations in a way that is conducive to healthy behavior. For instance, people who want to adopt a healthier diet can be advised to stick to a grocery list when shopping so that they do not end-up with a cart full of snacks. Similarly, people who want to stop smoking might want to stay out of pubs duning the first few weeks. Both strategies reduce exposure to seductive cues that are apt to evoke the unwanted behavior.

Secondly, situation modification concems efforts to reduce negative experience (e.g, asking the upstairs neighbor to tune down his music after midnight or buying earplugs). Situation modification can be achieved in health promotion activities, that is, environmental interventions to reduce situational cues that are likely to instigate action with detrimental health consequences: Consider, for example, the sheer number of fast food restaurants, commercials for unhealthy food products, and cigarette vending machines in bars. Besides limiting the number of motivationally salient cues, such interventions also ought to create environments that enable the execution of desired behavior by reducing the costs involved (Sallis, Bauman, \& Pratt, 1998). For example, by making it easier to go to the supermarket by bike than by car or by increasing the availability of showers in offices so that it is more convenient for employees to exercise during working hours.

Attentional deployment is used in attempts to neglect disturbing cues, for example by watching television instead of lying awake in bed, irritated by the upstairs noises. Feedback may present an excellent strategy for redirecting people's attention as it makes short-term, positive consequences of a particular behavior (e.g, exercising) more readily available, and thus limits the dominance of negative aspects.

Cognitive change relates to modifications in one's perception of the situation so that it no longer elicits a negative feeling. For instance, starting to think about the noise upstairs as a comforting background ture. Persuasion seems the primary means to induce cognitive change. Note that by considering persuasion as a means to induce cognitive change, its effectiveness can be assessed by changes in individuals' emotional responses to an object or behavior.

Finally, response modulation, refers to a change of initial response tendencies. For example, instead of going upstairs at 3:00 a.m. to get angry with the upstairs neighbor, you may savor a rancorous joy from deciding to wait until the next morning and wake him bright and early by playing loud music yourself at 7:00 am. The formation of implementation intentions has proven a successful means to modulate response tendencies. 
People who want to quit smoking for example can be asked to make an implementation intention to brush their teeth every time they feel like having a cigarette.

In general, the importance of situationally induced emotional influences on behavior, suggests that an important technique for health education is to have people mentally simulate the situations in which they are liable to be confronted with temptations. Subsequently, they should consider how they could deploy each of the emotion regulation strategies to their advantage. Such a procedure resembles inoculation techniques (e.g, Eagly \& Chaiken, 1993) that are used to strengthen certain beliefs. A mental simulation procedure to improve efforts at emotion control, however, exceeds beyond the stabilization of desirable cognitions.

Whether the strategies just described will ultimately prove beneficial is an open-ended question and is likely to depend on the particular problem at hand. Nevertheless, health education researchers may benefit greatly from improved understanding of emotionregulation processes and ways to influence them. Insights are likely to be derived from various psychological disciplines. Biological psychology, for one, may contribute by identifying the neural substrate of emotion-regulation capacities. Obviously, the previously discussed reciprocal pathways between prefrontal cortex and subcortical emotional brain structures mediate the potential for emotion-regulation (LeDoux, 1996; Maclean, 1990). Individual differences in the general capacity to influence emotional experience have indeed been related to the size of brain area's involved in the volitional control of behavior (Bush, Luu, \& Posner, 2000). Furthemore, cognitive and personality psychology may further elucidate the nature of individual differences underlying emotion-regulatory abilities, such as the capacity to verbally label emotional experience (Feldman Barret, Gross, Conner Christensen, \& Benvenuto, 2001), or individual variation in emotion-regulation strategies (Gohm, 2003; Gross \& John, 2003). Improving individuals' emotion-regulatory capacities may prove a very successful tool to health education practitioners in the years to come.

\section{CONSEQUENTIY...}

Two perspectives towards understanding human behavior figured prominently throughout the present dissertation. The first, also referred to as the reasoned-action perspective, considers behavior to be the result of an intentional decision based on a deliberative process of weighing perceived pro's and con's of acting. This perspective is formalized in expectancy-value models, such as the Theory of Planned Behavior (Ajzen, 1991; Ajzen \& Fishbein, 1980). Alternatively, we described the motivational perspective, in which behavior is portrayed as a goal-directed process (Austin \& Vancouver, 1996). Basically, this dissertation constitutes an attempt to integrate both perspectives. 


\section{Emotions as Goals}

We argued that previous attempts at integration failed because a clear conception about the nature and origin of the general goals underlying behavior is lacking. Based on arguments derived from evolutionary theory, we argued that the spectrum of human emotions might serve as an index to these goals (Cosmides \& Tooby, 2000). Hereto, we adopted a functional approach towards understanding the role of emotions in behavioral control (Izard \& Ackerman, 2000; Keltner \& Gross, 1999). According to the latter, emotions constitute the basic motivational systems, orchestrating action in accordance with environmental cues signaling the relevance of the situation to the attainment or frustration of these general goals.

We conducted two studies providing (further) support for the proposed association between emotions and goals. In Study 3.1, we assessed correlations between emotions and values. Values are another construct specifying the general goals underlying behavior (Schwarz, 1992). We found significant comelations between people's ratings of the frequency with which they experienced specific emotions in their daily lives and their ratings of importance of different values that ostensibly relate to the same goal. These results suggest that the more people strive to attain a particular goal (e.g., safety), the more they experience the particular emotion (e.g., fear) that, according to functional accounts, constitutes the primary motivational drive underlying behavior aimed to attain this goal. In Study 3.2 we demonstrated interactive effects between emotions and chronic activity if behavior-specific goals on behavioral decisions in a prisoner dilemma game. The occurrence of these interactions implies that emotional states and situational goal-activity affect behavior through the same underlying process, and thus provides further support for our conceptualization of emotions as general goals.

Finally, we argued that emotions are superior to other goal-constructs (e.g., values or needs) as indicators of general goals because psychophysiological measures provide an objective means to determine the occurrence of an emotional response, and neuroimaging techniques can be applied to differentiate between various distinct emotions. Consequently, emotions exceed the level of cognitive representation at which other goal constructs are assessed.

\section{The Integrated Perspective}

From an analysis of accumulated comments on the reasoned-action perspective it was concluded that expectancy-value models fail to account for the "energizing" or motivational properties of anticipated outcomes. Specifically, it was argued that nothing about outcome expectancies, described as perceived consequences of acting, necessitates the holder of that belief to act accordingly. It was proposed that only from considering the goal-related nature of outcome expectancies, their influence on behavioral decisions, as proposed by expectancy-value models, could be understood. Therefore, outcome expectancies were defined as intemal representations, derived from interactions of an individual with the environment, that specify how a particular act is conducive to or 
frustrates the attainment of the general goals underlying behavior. According to this integrated perspective, the behavioral impact of outcome expectancies is (at least partly) dependent upon the functioning of the basic motivational systems underlying behavior (see Figure 4.1).

It was hypothesized that goal-activation would affect the relation between outcome expectancies and behavior by influencing the accessibility or evaluation (in term of probability and valence) of goal-related outcome expectancies, or by moderating the impact of goal-related outcome expectancies on behavior. Additionally, the integrated perspective suggests the possibility of a direct effect of goal-activation on behavioral decisions, independent from reasoned-action processes. Results of two empirical studies, as well as several findings reported in the literature, provide some initial support for the integrated perspective. Generally, it was found that goal-activation increases the perceived likelihood of outcome expectancies that specify how the behavior under investigation relates to the particular goal. This result was limited, however, to anticipated outcomes that are not already strongly associated to the behavior. Furthermore, results indeed indicted goal-activation to directly affect behavior (-al intentions), independent form reasoned-action processes.

\section{Conclusions}

Based on the discussion and results of our studies as presented in this dissertation, we derived the following conclusions, pertaining to theoretical and empirical support for, and practical implications of the integrated perspective:

1. On a theoretical level, the introduction of goals improves the psychological validity of EV models by elucidating the processes by which the anticipation of behavioral outcomes causally underlies behavioral decisions.

2. Although the introduction of goals does not conflict with the considerations of Ajzen and Fishbein (e.g., 1980) to exclude distal variables from their model, empirical suppoit does not yet allow for the exact modeling of the integrated perspective. Further research is needed to investigate the hypothesized effects of goal-activation on reasoned-action processes.

3. Pertaining to practical implications, the integrated perspective may account for certain limitations of information-based approaches to health education that are not immediately apparent from current EV models. Nevertheless, it also suggests that information-based health education will be more effective if tailored to individual, behavioral, or situational differences in levels of goal-activity. Furthermore, it suggests that effective behavior-change methods may ensue from investigating methods to facilitate individuals' attempt at emotion-regulation 


\section{Towards a new metaphor...}

As mentioned from the start of this dissertation, different perspectives on behavior are usually associated with particular metaphors to illustrate the major tenets underlying a particular view. Given the provisional nature of the supporting evidence, it might be considered a bit premature to alreadly conceive of a full-blown metaphor capturing the tenets of the integrated perspective. Particularly since further research is still required to investigate the specific processes by which goal activation may influence deliberative processes in action planning and execution.

We nevertheless propose a simple allegory that conveys the major implication of the integrated perspective on behavior. According to ancient Greek dramatists, the universal human tragedy is that in spite of our limited capacities for predicting future consequences, we still remain responsible for our actions (see: Oatley \& Jenkins, 1996, p. 13). The integrated perspective recognizes this dualism between the premeditated and reactive forces instigating human action, and leads one to accept the boundaries of intentional control of behavior. All too often, we may be guided by reason, yet struck by our desires emanating from momentary emotional reactions: As was Eve when picking the apple - the forbidden fruit - from the tree of Knowledge in the Garden of Eden. Though fully awrare of the dire consequences of this act, by which she forfeited her immortality (talk about your detrimental health-consequencesl) and her placid life free of sorrow and pain, she still chose to satisfy her momentary desire.

The apparent schism in human nature was described beautifully by Alexander Pope in his Essay on Man (1733):

Know then thyself, presume not God to scan;

The proper study of mankind is Man.

Plac'd on this isthmus of a middle state,

A being darkly wise, and rudely great:

With too much knowledge for the Sceptic side,

With too much weakness for the Stoic's pride,

He hangs between; in doubt to act or rest;

In doubt to deem himself a God, or Beast;

In doubt his mind or body to prefer;

Born to die, and reas'ning but to err;

Alike in ignorance, his reason such,

Whether he thinks too little, or too much:

Chaos of Thought and Passion, all confused;

Still by himself abused, or disabused;

Created half to rise and half to fall;

Sole judge of Truth, in endless Error hurled:

The glory, jest and riddle of the world! 


\section{Appendix}

\section{Goal Priming by Semantic Discrepancy Primes}

In this Appendix $x^{5}$ we present four experimental studies in which we investigated the effectiveness of the goal-priming manipulation applied in Study 4.2 .

\section{INTRODUCTION}

In his auto-motive model, Bargh (1990) argues that goals can become activated by preconscious analysis of environmental information and may subsequently direct behavior without individual awareness, exactly like consciously intended goals do. Underlying this capacity is the process by which direct situation-to-goal links develop over the course of time from consistent and frequent conscious choice of a particular goal and subsequent action in a given situation According to the model, this intentional choice ultimately becomes automated in much the same way as during skill acquisition (e.g., learning to drive a car). This process ought to apply for proximal behavioral goals, as well as for general, higher-order goals or motives (Bargh, 1990; Bargh \& Chartrand, 1999). Several studies have provided support for the model. For instance, priming the goal to travel enhanced recognition of habitual travel modes (Aarts \& Dijksterhuis, 2000). Results from a classic study investigating the influence of (consciously held) memorization vs. impression-formation goals were replicated when these information-processing goals were primed without individual 
awareness (Chartrand \& Bargh, 1996). Also, priming mirrored the effects of chronic accessibility of (intrinsic and extrinsic) motivational orientations in terms of task-enjoyment and achievement as predicted by self-determination theory (Levesque \& Pelletier, 2000). Furthermore, in a series of expeniments, priming achievement goals appeared to increase individual performance on a variety of puzzle tasks (Bargh \& Gollwitzer, 1994), whereas priming cooperation goals increased collaboration among participants (Bargh, Gollwitzer, Lee-Chai, Barndollar, \& Trötschel, 2001).

A rare exception aside (Spencer, Fein, Wolfe, Fong, \& Dunn, 1998), most studies applied a semantic priming procedure (usually scrambled sentences; Srull \& Wyer, 1979) to supraliminally (ie., with individuals being aware of the stimulus material but not of the potential influence thereof) activate a particular goal. The rationale behind this procedure is that, like other knowledge constructs (such as attitudes or stereotypes), goals are mentally represented in associative networks (Anderson, 1983; Bargh \& Chartrand, 2000; Kruglanski, 1996) that consist of nodes representing the construct (e.g, the goal) and links specifying associations between constructs (e.g., between situational characteristics and goals, between related (sub) goals, or between goals and particular actions that constitute means to their attainment). Presentation of synonyms of a goal presumably activates the semantic representation of that goal, which in turn, is assumed to activate associated motivational and actional components through spreading of activation along the links in the network.

Given the sheer number of these studies, one may, all other things aside, safely infer that semantic priming has become a popular paradigm for testing claims on automatic goal activation. In this paper, however, we wish to address some traps of over-reliance on a successful paradigm. Our main point of concern is that although proof seems abundant, some aspects of semantic priming studies still warrant caution before they can be interpreted as providing conclusive evidence on the possibility of automatic goal activation as suggested by the auto-motive model. In the remainder of this appendix we develop the rationale for a semantic goal-priming procedure that is not based on goal symonyms and present the results of four studies indicating that, although this ought to be a theoretically sound way to activate goals, it nevertheless proved unsuccessful. Finally, considerations for automatic goal activation by semantic-priming techniques are discussed. It should be duly noted, however, that we do not intend to dispute the general idea of automatic goal activation or to question the results of the studies discussed above. We merely try to voice some concems about the utility of semantic-priming procedures for testing the general ideas of the auto-motive model.

\section{Three Routes to Automatic Goal Activation}

Goals can be defined as intemal representations of desired or anticipated outcomes or endstates (Austin \& Vancouver, 1996). As such, goals can be derived from a wide scope of individual needs, ranging from physiological set points to complex cognitive depictions of desired outcomes. Given this definition, a particular goal normally becomes activated to guide behavior in case the environment (internal or external) signals either (a) an 
opportunity to achieve some desired outcome (eg. an exam may present a way to display ones skills, thereby activating an achievement goal) or (b) when the environment signals a discrepancy between the current and an ideal or desired state (Higgins, 1997; Kruglanski, 1996). For instance, a bad grade may be an incentive to somehow restore one's self-esteem.

So far however, studies applying semantic priming techniques have mainly primed goals by (c) presenting goal-synonyms. However, as acknowledged by Bargh and Chartrand (1999, p. 472) "a direct test of this [the auto-motive] hypothesis [...] requires the [...] goalactivating stimuli to be semantically related to the situation, not to the goal itself; otherwise the environment-goal path is assumed but bypassed by the priming manipulation." Hence, a goal should also become activated by presentation of words related to situational features of the environment in which a particular goal is likely to be activated. Support for this assumption comes from but a single study (Bargh, Raymond, Pryor, \& Strack, 1995), in which presenting participants with prime words related to power, facilitated recognition of sexrelated words in male participants, but only for those with strong associations between power and sex. The basic idea of this study was that having power in a situation signals the opportunity to attain one's desired goal in that particular situation. Therefore, activating the concept of power likely increases the chances of selection and pursuit of that goal (in this case: sex).

As mentioned, it should also be possible to activate a goal by presenting words that signal situational features signaling a state of discrepancy (rather than opportunity) from that goal. To our best knowledge, however, this has not been previously investigated. Like opportunity signals, situational features of discrepancy should also be goal-specific

\section{Overview of the Present Studies.}

In a series of four experiments we investigated whether general goals can be activated by semantic priming, using stimuli describing general features of a state of discrepancy from that particular goal We aimed to prime two different goals, Personal Safety (PS; using words signaling imminent personal harm) and Cooperation ( $\mathrm{CO}$; using words describing characteristics of people in need of support).

We assessed the effects of this priming manipulation on a scope of variables related to different psychological processes that are supposed to be influenced by the activation of goals (Austin \& Vancouver, 1996; Kruglanski, 1996). Specifically, we assessed the influence of goal priming on four different outcome measures: self-reported feelings, perceived goalimportance, behavioral tendencies in a mixed-motive situation, and a reaction-time (RT) task We tested the following hypotheses: First, perceived discrepancy from an ideal state supposedly prompts negative feelings, ranging from general negative affect to specific feeling states (ie., emotions) that, according to both classic appraisal theories as well as recent functional accounts (e.g., Keltner \& Gross, 1999), are deemed characteristic for a particular need or goal. We expected goal priming to result in participants reporting more negative affect (for both PS and Co primes) and in higher reported intensity of goal-specific emotions like fear (for the PS prime) or concem (for the Co prime). Secondly, activating a goal should 
temporarily increase its subjective importance over others. We therefore expected that priming would result in participants rating either personal safety or cooperation-related goal constructs as more important than a neutral control group. To test this hypothesis we assessed participants' ratings of importance of values derived from Schwart' Value Survey (SVS). These values can be regarded as motivational constructs specifying general goals guiding people's actions across context and time (Schwartz, 1992). Thirdly, discrepancy from a desired state should prompt action aimed to eliminate that discrepancy. To investigate this we presented participants with a short description of a situation involving a motivational conflict between PS and Co goals, and subsequently asked them to rate the attractiveness of several behavioral responses to that situation, that either related to realizing a PS or a Co goal We expected prime-congruent effects for these ratings. Finally, goal activation is presumed to enhance processing efficiency of goal-relevant stimuli (Fazio, 1990). We investigated this effect by means of a lexical decision task in which RTs to neutral, PS-related and co-related words were assessed. We expected priming to facilitate recognition (i.e., to decrease response latencies) of words related to that goal.

As all experiments followed the same basic design and only differed regarding the dependent variable under investigation, methods and results are combined into two overall sections.

\section{METHOD}

Participants and Procedure. A total number of 196 students (82 men, 114 women), enrolled in introductory courses in psychology and health-sciences at the University of Maastricht, the Netherlands, participated in (one of) the experiments. Age ranged from 17 to 33 years $(M=19.3)$. Participants received a monetary reward of $€ 5$.

Participants were invited to the laboratory in groups from 1 to 6 in each session. Upon arrival, they were informed that they would participate in two unrelated studies. The first study, which was presented as a language-ability test, was in fact the goal-priming manipulation. Participants were randomly assigned to either the PS-goal priming condition, the Co-goal priming condition or to a neutral Control group (though with the restriction that approximately equal numbers were represented in each). After written consent was provided, they were handed out the materials for the priming manipulation in an envelope so the experimenter was kept unaware of participants' priming condition. They were then seated in individual cubicles during completion of both tasks. After finishing the priming manipulation they were given the materials with written instructions for the other task (see below) in another envelope. The average duration of the experiment was about $25 \mathrm{~min}$. When finished, participants were individually debriefed using a funneled questionnaire protocol (cf. Bargh \& Chartrand, 1996), thanked, and dismissed after reoeiving their money.

Goal Priming Manipulation. We used a Scrambled Sentence Test (Srull \& Wyer, 1979) in which participants constructed grammatically correct four-word sentences from randomorder sequences of five words. The test constituted of a total number of 30 sentences, 20 of 
which contained words or described situations implying a state of discrepancy from the goals of PS or Co. Primes in the PS condition were: riot, violent, ominous, danger, risk, phobia, frightening, ternifying, hostile, panic war, wounded, waming, assault, murder, death, alam, flee, and running (away). For the Co priming condition, the primes were: helpless, fragile, powerless, unfair, suffer, weak, vulnerable, threatened, harassed, defenseless, deserted, poverty, need, dearth, abandoned, alone, frail, unprotected, and cast off. Sentences in the PS condition were all stated in the first person to enhance self-relevance, whereas sentences in the Co condition were all stated in third person to convey concern for another. All other words and all words in the Control condition were neutral with respect to the type of goal.

Experimental Tasks. The four experiments constituted an assessment of subjective feelings, ratings of goal importance, behavioral tendencies in a mixed-motive situation, and a reaction-time task, respectively.

Self-Reported Feelings. In this experiment, participants ( 27 men and 26 women, mean age $=19.2$ years) were asked to report the intensity with which they experienced, at this very moment, each of 24 feelings and emotions, by placing a cross on a VAS-scale, following recommendations by Larsen \& Fredrickson (1999) for assessment of specific, discrete emotions. The length of the line from the left-end (anchored: not at all) to the cross was used as a measure of intensity. The total length of the line was $10 \mathrm{~cm}$. The right-end side of the scale was an arrowhead marked with $\mathbf{a}+$ sign, illustrating increasing intensity'. Three items measuring general negative affect (unpleasant, sad, and depressed) were averaged into one measure of negative affect $(\alpha=.70)$. Furthermore, two composite scales measured PSemotions (nervous, tensed, and edgy; $\alpha=81$ ) and Co-motions (concem, sympathy, and pity; $\alpha$ $=.83$ ) respectively. The other items were added as fillers (e.g., cunious, bored, angry).

Goal Importance Participants ( 26 men and 27 women, mean age $=19.2$ years) were asked to rate a selection of 24 values items for perceived importance on a 9-point scale ranging from 1 (not at all important) to 9 (extremely important). The oniginal SVS consists of 56 items that have been shown in previous analyses to fall into 10 higher-order categories that can be distinguished on the basis of their motivational goals (Schwartz, 1992). The selected value-items in our study belonged to the higher-order categonies of Security (eg.: safety, social order, healthy) related to the primed PS-goal, and Benevolence (e.g.: helpful, responsible, loyal) related to the primed Co-goal, and were replenished with a few filler items, pertaining to other, unrelated categories. As alpha coefficients were sufficiently high for both the Safety $(\alpha=86)$ and the Benevolence $(\alpha=.75)$ category, value item scores were averaged into single measures.

Behavioral Tendencies. Participants (26 men and 26 women, mean age $=19.1$ years) were presented with a short description of a situation entailing a motivational conflict between PS and Co goals. Participants were asked to imagine themselves witnessing an act of random violence and to subsequently rate their preference for three possible behavioral responses to the situation, on a 10-point scall, ranging from 1 (not at all preferred) to 10 (very much preferred). The behavioral responses were selected to present means to realize either the PS-goal (nunning away), the Co-goal (help the victim), or neither (punish the perpetrators). 


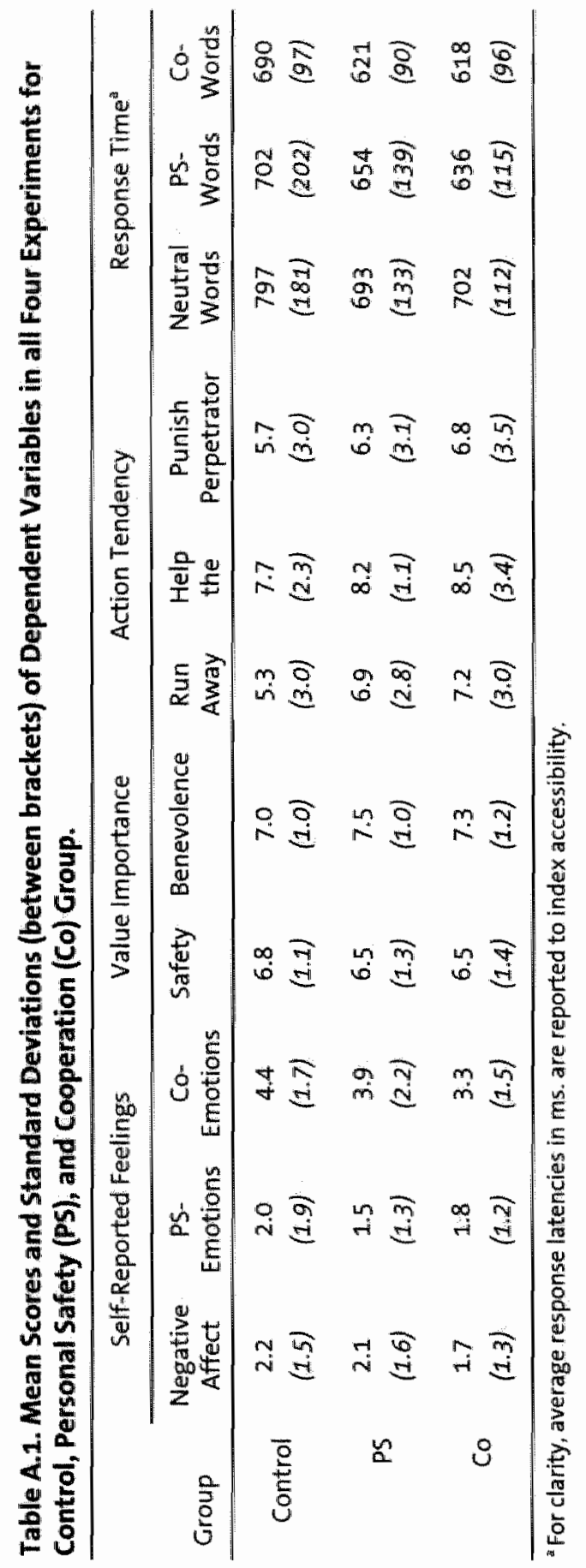


Reaction time Finally, in order to test whether goal priming enhanced processing efficiency of goal-relevant stimuli, we performed a lexical decision task using E-Prime version 1.1. Participants ( 3 men and 35 women, mean age $=19.9$ years) were seated in front of a computer screen. They were instructed to decide as accurately and rapidly as possible, whether the sequences of letters they were exposed to represented a word in the Dutch language (in which case they had to press the q-key on the numerical part of a standard QWERTY keyboard) or a non-word (in which case they had to press the p-key). Word stimuli consisted of four words related to the PS-goal (unsafe, dangerous, threatening, and fear), four words related to the Co-goal (help, care, support, and concemed), as well as eight reference words that were neutral with respect to the two goals and were matched for word-length and frequency of occurrence in the Dutch language. In addition, 16 non-words were included. After a practice session using 5 words and 5 non-words, all 32 words were presented in a single randomized block, during which response latencies and response accuracy (correct or incorrect identification of words and non-words) were recorded.

\section{RESULTS}

Mean scores and standard deviations of the three experimental groups (Control, PS, and Co) on each of the dependent measures in all four experiments are summarized in Table A.1.

\section{Self-Reported Feelings}

One participant indicated suspicion of the content of the priming manipulation and was excluded from analysis. A OneWay ANOVA with prime condition as the only between-group variable, did not show a significant effect of goal priming on self-reported intensity of negative affect, $F(2,52)=.59, p=.56$, PS-related emotions, $F(2,52)=.40, p=.67$, or Co-related emotions, $F(2,52)=1.54, p=.22$.

\section{Perceived Goal Importance}

Neither the perceived importance of Benevolence $(F(2,52)=.88, p=42)$, nor of Safety values $(F(2,52)=.42, p=66)$, differed between experimental groups.

\section{Behavioral Tendencies}

Priming effects on average preference ratings for each of the pre-specified behavioral response tendencies to the mixed-motive situation in Experiment 3 were analyzed using the same OneWay procedure. Although the omnibus test of the main effect did not reveal a. main affect of goal priming on the PS-related response $(F(2,51)=1.92, p=.16)$, a post-hoc test (Tukey's HSD) indicated a marginally significant difference $(p=.08$, one-tailed), suggesting that the tendency to run away was moderately higher in the Co than in the Control group, which was contrary to our expectations. No prime effects were found for the Co-related (helping the victim; $F(2,51)=1.21, p=.31$ ) or the other behavioral response (punishing the perpetrators; $F(2,51)=.59, p=.56)$. 


\section{Reaction Times}

Following guidelines proposed by Fazio (1990), raw RT latencies were logarithmized in order to approximate them to the normal distribution. Only RTs for accurate trials (ie. correct responses to words and non-words) were used for statistical analysis. Furthermore, all RTs outside \pm 3 sd's of the population mean were treated as outliers. Mean reaction times (mRT) for safety-related, cooperation-related, and neutral words in each of the priming conditions are reported in Table 1.

A OneWay ANOVA of $m R T$ of neutral words by prime condition did not reveal significant differences between prime groups, $F(2,35)=191, p=.16$, so there was no reason to assume that differences in target latencies should be attributed to different decision criteria across conditions. In order to test for goal priming effects on RTs we performed a OneWay ANOVA of $\mathrm{mRT}$ of both safety- and cooperation-related words by prime. Result showed a marginal overall effect of prime condition on mRT of cooperation related words $F(2,35)=2.49$, $p=.09$ ) that was further qualified by a significant contrast $\mathrm{C} 1(\mathrm{PS} ; \mathrm{Co} ; \mathrm{C}=-1 ;-1 ; 2)$ companing the control with both goal-prime groups, $t(35)=2.22, p=.03$, suggesting that both $\mathrm{PS}$ and $\mathrm{Co}$ primed participants responded faster to cooperation related words than participants in the Control group. No difference was found, however, between PS and Co prime groups, C2 (PS; $\mathrm{Co} ; \mathrm{C}=-1 ; 1 ; 0), t(35)=.20, p=.84$. No significant overall differences were found between groups on mRT of safety related words $F(2,35)=, 44, p=.65$. Including mRT of neutral words into the analysis as a covariate in order to control for possible confounding by individual differences in response latencies did not change the results (all $F s<1$ ).

\section{Discussion}

The data did not support any of the expected effects of our priming manipulation on selfreported feelings, perceived goal-importance, and behavioral tendencies. The only indication of any prime effect was a decrease in response latencies to cooperation related words in both PS and Co prime groups. In itself, this is too modest a result to support claims of automatic goal activation by semantic discrepancy primes.

Naturally, attempting to interpret non-significant data is a hazardous enterprise. In case a test of a model within a single paradigm fails to reject the null-hypothesis, this cannot be considered as disconfirmation of the model, but (merely) reflects either (a) flawed design or measurement or, as we argue, (b) limitations of the particular paradigm as a means to verify specific predictions of the model. By carefully scrutinizing several altemative explanations, we hope to illustrate that the former is implausible and results of the present studies can indeed be best interpreted as denying the possibility for goal priming by means of goal-discrepant semantic stimuli.

First of all, we wish to emphasize the (purposeful) resemblance of the basic design features of our studies to other studies cited in this paper - that did successfully activate goals by semantic priming - in terms of (a) the demographic characteristics of the research 
population, (b) the procedures followed in the lab, (c) the content of the cover story by which the prime was introduced, as well as (d) the debriefing procedures, and (e) the internal structure of the prime (scrambled sentences).

We admit that the experimental manipulation applied in our research has its limitations. First of all, it applied the same stimulus configuration in every test. It should, however, be noted that each word in the set constitutes a unique stimulus and a set of 20 words, therefore, encompasses a rather extensive collection of stimuli We therefore trust that our set comprises a comprehensive collection of discrepancy signals of both the PS and the Co goal (This point will be addressed at a later point in this discussion) Another critique to our prime, would argue that it might have been too strong, thereby eliciting contrast effects ( $\mathrm{eg}$, Higgins, 1996). Yet obviously, the data do not support this explanation.

Thirdly, we would like to address possible comments that could be raised against the targets of our manipulation, the goals of PS en Co. One may be inclined to explain the failure of our priming manipulation by referring to the well-known fact that automatic effects of primed goals or actions are inhibited in case of associated costs or clear negative consequences (Macrae \& Johnston, 1998). Although the target words in our priming manipulation have obvious negative connotations, it should not be overlooked that they were not the actual goals to be primed, but referred only indirectly (as situational features) to these goals, which themselves constituted desirable ends. Another obvious shortcoming would be if these goals were of no concern to our research population. It would, for example, have been useless to activate goals like 'saving household energy' in students who (in the Netherlands) usually pay rent with energy costs already included. Goals like PS and Co, however, can be deemed universal given their presence in various taxonomies specifying general goals of human behavior (see: Austin \& Vancouver, 1996). One might subsequently argue that being universal, these goals are chronically accessible and therefore insusceptible to additional priming influences (Higgins, 1996). Several studies have nevertheless indicated that individuals differ in levels of chronic accessibility of general goals, and that such differences are a major source of personality differences (e.g., Schwartz, 1992). Priming effects can thus still be expected to affect these goals. Moreover, the automotive model itself states that principles of automatic activation should equally apply for proximate, behavioral goals as well as general goals (Bargh, 1990).

Finally, criticism could concentrate on the nature of the dependent variables we chose to assess goal activation. Like the nature of the goals, one might argue that our dependent measures did not present relevant means of goal attainment to our research population (e.g. Kruglanski, et al, 2002). Nevertheless, this would only explain the lack of influence of our manipulation on the behavioral-intention measure. We assessed goal-priming effects on an exhaustive collection of variables that do not reflect means of goal attainment but rather assess autonomic processes (emotion, perception) that are not subject to individual choice.

Acknowledging the speculative nature of the considerations mentioned above, we believe limitations of the research paradigm, rather than the design, to provide a more plausible account for our results. Several characteristics of associative network models of 
semantic memory (Anderson, 1983) may account for the apparent insensitivity of (general) goals to sernantic primes signaling discrepant states. First of all, subliminal or supraliminal priming manipulations tap implicit processes (spreading of activation in associative networks): Research on ironic processes (e.g. Gilbert, 1991) has shown that implicit processes are incapable of dealing with negations, as illustrated by the dassic example of how trying not to think about a white bear exerts the exact opposite effect. Similarly, implicit processes may not always be capable of interpreting discrepancy-primes (e.g., danger) as negations of a goal state (not-safe) and such primes may therefore be unable to activate the intended. motivational tendency. Another problem may be that only a few discrepancy signals are generally associated with a particular goal. For whereas synonyms unambiguously refer to the same goal for each individual, idiosyncratic differences are likely for what qualifies as a situational characteristic associated with a particular goal. Thus, the scope of semantic primes that still qualify as ostensibly universal discrepancy signals by which the general goals of PS en Co could be activated is quite limited. We already mentioned this in addressing limitations of our priming manipulation. In our opinion then, this limitation bolsters, rather than refutes our position that general goals cannot be activated by semantic primes signaling situations of goal-discrepancy, as this research question inherently narrows the scope of possible semantic stimuli. (Nevertheless, we admit that other types of discrepancy primes may prove effective in activating goals, a point that we return to at the end of this discussion) Furthermore, associative networks are hierarchically organized, with more abstract concepts (general goals) having more links than subordinate concepts (specific goals). As a result, activation due to priming of abstract concepts is more "spread out", a phenomenon also called the "fan-effect", and hence, the impact of priming general goals will exert less pronounced effects than priming specific ones would (Markman, 1999). Finally, links between a subordinate (e.g. sparrow) and a parent concept (e.g, bird) are usually stronger than vice-versa. Therefore, detecting priming effects of general goals on more specific associated concepts (e.g., concrete values, particular behavioral acts) will be more difficult as opposed to finding such behavioral effects after priming a goal on a more similar level.

Naturally, the latter two explanations are not specifically related to manipulations using goal-discrepant primes, but rather concern common difficulties related to the priming of general - as opposed to more specific - goals. Results of studies in which specific goals (e.g. traveling) have been primed can still be interpreted in terms of activation of behavioral schemata General goals, however, constitute those that cannot be reached in a single act or in a single occasion and automatic activation effects are thus less vulnerable to accounts of activated behavioral representations. The only general goals, however, that were automatically activated using semantic priming procedures are the goals of cooperation (Bargh, et al., 2001) and achievement (Bargh \& Gollwitzer, 1994). Notably, operation of a true motivational process underlying the effects of automatic goal activation has only been documented for the achievement goal. Assuming the role of the devil's advocate, one could argue that rather than an achievement goal, this priming manipulation merely activated 
general characteristics of motivated behavior, using target words like strive, achieve(l), succeed, compete, etcetera. Furthermore, given the critique in Footnote 1 and the results of the present study, there seems to be at least some ground for suspicion conceming the possibility to activate general goals by semantic priming techniques.

Apart from that, there are additional reasons to explore other venues for testing the automotive model One could also question whether the results of semantic priming techniques in controlled laboratory settings, allow for strong generalizations on the ubiquity of automatic goal activation at all. First of all, the ecological validity of the dependent measures used to assess automatic effects do not allow for wide generalization of results (Sheeran, 2002). Priming studies have usually relied on behaviors that are much less involving for participants than the ones investigated in applications of intention-based theories. Therefore, more research should address the relative influence and interplay of automatic and intentional processes in determining complex behavior. Secondly, the ecological validity of the stimulus appears limited. Manipulation by presenting goalsynonyms is acknowledged to bypass the actual cause of goal activation, which lies in environmental features. Regarding the limited evidence for opportunity related prime words and the results of the present study one should wonder whether semantic primes can be used to mimic the effects of these environmental features anyhow.

To our opinion, these present compelling arguments to test automatic goal activation effects by using other priming techniques. For instance, inducing specific goal-related emotional states by autobiographical recall procedures may present a good alternative. Not only does such a manipulation more closely resemble real phenomena that induce goal states, it has the additional benefit of being idiosyncratic by nature. If automatic activation of goals proves possible using this type of manipulation, this would furthermore imply that discrepancy signals (negative emotions) can automatically induce general goals and would thus support our present explanation of the results in terms of semantic memory processes.

Besides instigating discourse on the matters discussed before, we hope our data may contribute to future meta-analyses on the effects of goal priming methods. We have no aspirations of drawing firm conclusions from the results of our study, and do not challenge the idea of automatic goal activation, but given these considerations, we think it may be time to start looking beyond the scope of a single paradigm. 


\section{Footnotes}

"The plural "we" will be used throughout this dissertation in acknowiedgment of its realization being the result of a collaborative endeavour.

2 This paragraph is based on: Neiissen, R. M. A., Dijker, A. J., and De Vries, N. K. (2004). Integrating Motivational Constructs: Assessing Relations between Values and Emotions. Manuscript under review (for over a year) with Cognition and Emotion.

${ }^{3}$ This paragraph is based on: Nelissen, R. M. A., Dijker, A. J, and De Vries, N. K. (2005). How to Tum a Hawk into a Dove and vice versa: Interactions between Emotions and Goals in a GiveSome Dilemma Game. Manuscript under review with The Joumal of Experimental Social Psychology.

${ }^{4}$ Naumann and Richter (2000) investigated the usefulness of response latencies as indicators of the accessibility of attitude-relevant knowledge. Items were evaluative statements concerning different topics in the domain of social welfare, with an average length of 11 words. E.g. "social welfare is important for society". Participants were asked to indicate their" (dis)agreement on these statements. Results of this study suggested that response-latency measures can be used as measures of accessibility even if stimuli are semantically or syntactically complex, rather than single-item stimuli. Specifically, response latencies were found to be substantially correlated $\left(r^{\prime} s>4\right)$ with the number of times each item was mentioned in an interview on (the consequences of) social welfare.

"This appendix is based on: Nelissen, R M. A. Dijker, A. J, and De Vries, N. K. (2004). Limitations of Semantic Priming Procedures for Automatic Goal-Activation. Manuscript accepted for publication in Psychological Reports.

' One could argue that power is not a goal-specific situational feature but rather a general personal experience that enhances pursuit of whichever goal is activated at the moment, like for instance, feelings of personal competence, perceived control, or self-esteem.

${ }^{7}$ As we did not anticipate our priming manipulations to elicit strong emotional experiences, we expected extreme anchors to reduce between subject variance in response. 
क्येक्षिकी

s. 


\section{References}

Aarts, $\mathbb{H}_{\text {, \& }}$ Dijksterhuis, A (2000). Habits as knowledge structures: Automaticity in goal-directed behavior. Joumal of Personality and Social Psychology, 78: 53-63.

Aarts, H. Verplanken, B., \& van Kruppenberg A (1998). Predicting behavior from actions in the past: Repeated decision making or a matter of habit? Joumal of Applied Social Psychology, 28: 13551374.

Abe, J. A A., \& Izard, C. E (1999). The Developmental Functions of Emotions: An Analysis in Terms of Differential Emotions Theory Cognition and Emotion, 13: 523-549.

Abelson, R. P., \& Prentice, D. A (1997). Contrast tests of interaction hypotheses. Psychological Methods. 2: 315-328.

Abraham, C., \& Sheeran, P. (2003). Implications of goal theories for the theories of reasoned action and planned behavior. Cunrent Psychology Developmental Leaming, Personality, Social 22: 264-280.

Abraham, $C_{n}$ Sheeran, $P_{\text {, }}$ \& Johnston, M (1998). From health beliefs to self-regulation: Theoretical advances in the psychology of action control. Psychology and Health, 13:569-591.

Ajzen, L (2002a). Perceived behavioral control, self-efficacy, locus of control, and the theory of planned behavior. Joumal of Applied Social Psychology, 32: 665-683.

Ajzen 1 (2002b). Residual effects of past on later behavior: Habitual and reasoned action perspectives. Personality and Social Psychology/Review, 6: 107-122.

Ajzen, L (1991). The theory of planned behaviour. Organizational Behaviour and Human Decision Processes, 50: 179-211.

Ajzen, $L_{1}$ \& Fishbein, $M$ (2004). Questions raised by a reasoned action approach: comment on Ogden (2c03). Health Psychology; 23:431-434.

Ajzen, $L_{2}$ \& Fishbeir, $M(1980)$. Understanding Attitudes and Predicting Social Behavior. Englewood. Cliffs, N: Prentice-Hall. 
Allport; G. W. (1935). Attitudes. In C. M. Murchison (Ed), Handbook of Social Psychology (pp. 789-844). Worcester, MA: Clark University Press.

Anderson, J. R. (1983). The Architecture of Cognition. Cambridge, MA: Harvard University Press.

Amitage, C. I. \& Chnistian, J. (2003). From attitudes to behavior: basic and applied research on the theory of planned behavior. Current Psychology: Developmental, Leaming, Personality, Social, 22: 187-195.

Anmitage, $C$. I, \& Conner, M (2000), Social cognition models and health behavior: A structured review: Psychology \& Health, 15:173-189.

Anmitage, $C .1,8 x$ Conner, $M(2001)$. Efficacy of the theory of planned behaviour: A meta-analytic review. British Joumal of Social Psychology, 40:471-499.

Atkinson, J. W. (1980). Old and new concepts of how expected consequences influence actions. In F.M. Toates \& T. R Halliday (Eds.j, Analysis of Motivational Processes (pp. 17-52). London: Academic Press.

Austin, J. T., \& Vancouver, J. B. (1996). Goal constructs in psychology: structure, process, and content. Psychological Bulletin, 120: 338-375.

Bagozzi, R. P. (1992). The self-regulation of attitudes, intentions, and behavior. Social Psychology Quarterly, 55:178-204.

Bagozzi, R. P., Bergami M, \& Leone, L (2003). Herarchical representations of motives in goal setting. Joumal of Applied Psychology, 88:915-943.

Balleine, B, \& Dickinson, A (1998). Consciousness - the interface between affect and cognition. In J. Cormwell (Ed), Consciousness and human identity (pp. 57-85). Oxford: Oxford University Press.

Bandura, A (2001): Social cognitive theory: An agentic perspective. Annual Review of Psychology, 52: 126.

Bandura, A. (1986). Social Foundations of Thought and Action. A Social Cognitive Theory. NY: PrentioeHall

Barbas, H (1995). Anatomic Basis of Cognitive-Emotional Interactions in the Primate Prefrontal Cortex. Neuroscience and Biobehavioral Reviews, 19:499-510.

Bardi, A, \& Schwartz S. H (2003). Values and behavion, Strength and structure of relations. Personality and Social Psychology Bulletin, 29: 1207-1220.

Bargh, J. A (1990). Auto-Motives: Preconscious deteminants of social interaction. In E. T. Higgins \& $\mathbb{R}$ M Sorrentino (Eds), Handbook of Motivation and Cognition (Vol 2:93-130). NY: Guilford Press:

Bargh, J. A, \& Chartrand, T. L. (2000). The mind in the middle: A practical guide to priming and automaticity research in $\mathrm{H}$ T. Reis \& $\mathrm{C}$ M Judd (Eds), Handbook of research methods in social and personality psychology (pp. 253-285). Cambridge: Cambridge University Press.

Bargh, J. A. \& Chartrand, T. L. (1999). The unbearable automaticity of being American Psychologist, 54: 462-492.

Bargh, J. A. \& Ferguson, M. J. (2000). Beyond behavionism, on the automaticity of higher mental processes. Psychological Bulletin, 126: 925-945.

Bargh, J. A, \& Gollwitzer, P. M (1994). Environmental control of goal-directed action. Automatic and strategic contingencies between situations and behavior. Nebraska symposium on Motivation, 41: $71-124$.

Bargh, J. A, Gollwitzer, P. M, Lee-Chai, A, Bamdollar, K, \& Trötschel, R (2001). The automated will. Nonconscious activation and pursuit of behavioral goals. Joumal of Personality and Social Psychology, 81: 1014-1027. 
Bargh, J A, Lombardi, W. I., \& Higgins, E. T. (1988). Automaticity of chronically acoessible constructs in person $x$ situation effects on person perception: It's just a matter of time Joumal of Personality and Sacial Psychology, 55: 599-605.

Bargh, J. A, Raymond, P. Pryor, J, \& Strack, F. (1995). Attractiveness of the underling: An automatic power $\rightarrow$ sex association and its consequences for sexual harassment and aggression Joumal of Personality and Social Psychology, 68: 768-761.

Baron, R. M, \& Kenny, D. A. (1986). The moderator-mediator wariable distnction in social psychological research: Conceptual, strategic, and statistical considerations. Joumal of Personality and Social Psychology, 51: 1173-1182.

Barsalou, L (1985). Ideals, central tendency, and frequency of instantiation as detemunants of graded structure categonies. Joumal of Experimental Psychology: Leaming, Memony, and Cognition, 11 629-654.

Batson, C D. \& Moran, T. (1999). Empathy-induced altruismin a prisoner's dilermma European Joumal of Social Psychology, 29: 909924.

Baumeister, R F., Bratslavsky, E, Muraven, M, \& Tice, D. M. (1998). Ego Depletion. Is the active self a limited resource? Joumal of Personality and Social Psychology, 74: 1252-1265.

Bechara, A., Damasio, H, Tranel, D, \& Damasio, A R (1997). Deciding advantageously before knowing the advantageous strategy. Science, 275: 1293-1295.

Behrendt, R-P. (2004). A neuroanatomical model of passivity phenomena. Consciousness and Cognition, 13: 579-609.

Ben-ZeEv, A (2000). The Subtlety of the Emotion. Cambridge, MA:MIT Press.

Berkowitz, L (1989). Frustration-aggression hypothesis: examination and reformulation. Psychological Bulletin, 106: 59-73.

Bindra, D. (1959). Motivation:Asystematic reinterpretation New York: Ronald Press.

Bower, G. H (1981). Mood and mernory. Amerioan Psychologist, 36: 129-148.

Brown, J. D, Dutton, K A, \& Cook, K E (2001). From the Top Down Self-Esteem and Self-Evaluation. Cognition and Emotion, 15:615-631.

Brug, J, Schaalma, H, Kok, G, Meertens, R. M, \& van der Molen, H. T. (2001). Gezondheidswoonlichting en Gedragswerandening: Een PlanmatigeAanpak. Assen, the Netherlands: Van Gorcum

Buck, R (1999). The Biological Affects: A Typology Pychological Review, 106: 301-336.

Buller, D. J. (2002). Defreuding evolutionary psychology: Adaptation and human motivation. Retrieved march $25,2002_{p}$ from http//oogprints.sotonacuk/documents/disko/00/00/03/26/cogoo000326-00/defreudhtiml

Buller, D. J, \& Hardcastle, V. G. (2000) Evolutionary psychology, meet developmental neurobiology: Against promiscuous modularity Brain and Mind, 1: 307-325.

Burbach, J. P. H. (2000): Genetic pathways in the developmental specification of hypothalamic neuropeptide and midbrain catecholamine systems. European joumal of pharmacology, 405: 55-62.

Bush, G, Iuu, P. \& Posner, M (2000). Cognitive and Emotional Influences in Anterior Cingulate Cortex. Trends in Cognitive Sciences, 4: 215-222.

Buss, D. M (1999). The Evolutionary Psychology of Human Social Strategies. In E. T. Higgins \&x A W. Kruglanski (Eds), Social Psychology: Handbook of Basic Principles (Vol 1, pp. 3-38). New York: Guilford Press. 
Cacioppo, I.T. Bentson, G. G, Larsen, J. T, Poehlmann, K. M, \& Ito, T. A. (2000). The psychophysiology of emotion In M Lewis \& J. M Haviland-Jones (Eds.), The handbook of emotions (pp. 173-191). NY: Guilford Press.

Cacioppo, J. T. \& Petty, R. E. (1982). The need for cognition. Joumal of Personality and Social Psychology. 42. $116-131$

Camerer, C.F. (2003). Strategizing in the brain. Science, 300: 1673-1675.

Carver, CS, \& Scheier, M. F (1998); On the self-regulation of behavior Cambridge: Cambridge University Press.

Carver, C. S. \& Scheier, M E. (1990). Origins and functions of positive and negative affect: A controlprocess view. Psychological Review, 97: 19-35.

Chartrand, T. L, \& Bargh, J. A (1996). Automatic activation of impression formation and memorization goals: Nonconscious goal priming reproduces effects of explicit task instructions. Joumal of Personality and Social Psychology, 71: 464-478.

Chulef, A. S., Read, S. J., \& Walsh, D. A. (2001). A Hierarchical Taxonomy of Human Goals. Motrivation and Emotion, 25: 191-232.

Cohen, L M. MeCarthy, D. M. Brown, S. A., \& Myers, M. G. (2002). Negative affect combines with smoking outcome expectancies to predict smoking behavior over time. Psychalogy of Addictive Behaviors, 16: $91-97$.

Colman, A M (2003). Cooperation, psychological game theory, and limitations of rationality in social interaction. Behavioral and Brain Sciences, 26: 139-198.

Conner, M, \& Amitage, C. J. (1998). Extending the theory of planned behaviour. Jounal of Applied Social Psychology, 28: 1429-1464.

Conner, M, Norman, $\mathrm{P}$, \& Bell, R (2002). The theory of planned behavior and healthy eating. Health Psychology, 21: 194-201.

Cooke, R, \& Sheeran, P. (2004). Moderation of cognition-intention and cognition-behavior relations: a meta-analysis of properties of variables from the theory of planned behavior. British Joumal of Social Psychology, 43: 159-186.

Cosmides, L, \& Tooby, J. (2000). Evolutionary Psychology and the Emotions. In M Lewis \& J. M Haviland-Jones (Eds.); Handbook of Emotions (2 ed, pp. 91-115). New York: Guilford Press.

Cosminides, Lx \& Tooby, J. (1987). From Evolution to Behavior: Evolutionary Psychology as the Missing Link In J. Dupré (Ed), The Latest on the Best: Essays on Evolution and - (pp. 277-306). Cambridge: MTT Press.

Damasio, A R. (2000). The feeling of what happens. London: William Heinemann

Damasio, A. R. (1994). Descartes' emor. Emotion, reason, and the human brain. New York: Putnam.

Danner, D. D., Snowdon, D. A. \& Friesen, W. V. (2001): Fositive Emotions in Early life and Longevity: Findings from the Nun Study. Joumal of Personality and Social Psychology, 80:804-813.

Darwin, C. (1872). The expression of the emotions in man and animals. London: Murray.

Darwin, C. (1859). On the origin of species by means of natural selection. Iondon: Murray.

Davidson, R. J. (1998). Affective Style and Affective Disorders: Perspectives from Affective Neuroscience. Cognition and Emotion, 12: 307-330.

Dawkins, R. (1986). The blind watchmaker. New York: Norton.

De Cremer, D. \& Van Lange, F. A. M (2001). Why prosocials exhibit greater cooperation than proselfs: the roles of social responsibility and reciprocity. European Joumal of Personality, 15:5-18. 
De Vet, E, De Nooijer, I, De Vries, N. K, \& Brug, J. (2005). The transtheoretical stages of change and frunt intake: Real or pseudostages? Manuscript Submitted for Publication

De Vries, H. Backbier, E, Kok, G, \& Dijkstra, M. (1995). The impact of social influences in the context of attitude, self-efficacy; and previous behavior as predictors of smoking onset boumal of Applied Social Psychology, 25: 237-257.

De Vnies, H, \& Brug, J. (1999). Computer-tailored interventions motivating people to adopt healthpromoting behaviours: Introduction to a new approach. Patient Education and Counseling, 36: 99-105.

Derryberry, D, \& Reed, M A (1996). Regulatory Processes and the Development of Cognitive Representations. Development and Psychopathology, 8: 215-234.

Denyberry, D., \& Reed, M A (1994). Temperament and the Self-Organization of Personality. Development and PSychopathology, 6:653-676.

DeSteno, D. Petty, R E, Rucker, D. D. Wegener, D. T., \& Bravernan, J. (2004). Discrete emotions and persuasion: the role of emotion-induced expectancies Joumal of Personality and Social Psychology, 86:43-56.

DeSteno, D. Petty, R E. Wegener, D. T, \& Rucker, D. D. (2000). Beyond valence in the perception of likelyhood: the role of emotion specificity. Joumal of Personality and Social Psychology, 78: 397416.

Dij]ker, A. J. (2001). Hoe Cognitie en Motivatie Gedrag Veroorzaken: Een alternatief woor mentalisme en hedonisme. In D. A Stapel \& J. van der Pligt (Eds), Een Tijd van Koomen. Amsterdam: Vossiuspers UvA.

Domjan, M, Cusato, B, \& Villareal, R (2000). Pavlovian feed-forward mechanisms in the control of social behavior. Behavioral and Brain Sciences, 23: 235-282.

Eagly, A. H, \& Chaiken, S. (1998). Attitude Structure and Function. In D. Gilbert, S. T. Fiske \& G. Lindzey (Eds.), The Handbook of Social Psychology. NY: McGraw-Hill.

Eagly, A. H, \& Chaiken, S. (1993). The Psychology of Attitudes. Orlando, FL Harcourt Brace.

Edwards, D. C. (1999). Motivation \& Emotion: Evolutionary. Psychological, Cognitive and Social Influences (1 ed Vol 3). Thousand Oaks: Sage Publications.

Edwards, W. (1954). The theory of decision making. Psychological Bulletin, 51:380-417.

Eisenberg N. (2000). Empathy and sympathy. In M Lewis \& J. M. Haviland-Jones (Eds.), Handbook of emotions (pp 677-691). New York Guilford Press.

Ekman, P. (1993). Facial Expression and Emotion. American Psychologist, 48: 384-392.

Elaman, P. (1992). An Argument for Basic Ernotions. Cognition and Emotion, 6: 169-200.

Elliot, A I, \& Thrash, T. M (2002). Approach-avoidance motivation in personality: approach and avoidance temperaments and goals. Joumal of Personality and Social Psychology, 82:804-818.

Evans, ]. S. B. T, \& Over, D. E (1997). Are people rational? Yes, no and sometimes. The Psychologist 10: 403-406.

Evans, $M$. G. (1991). The problem of analyzing multiplicative composites. American Psychologist, 46: 6m 15

Fazio, R H (2001). On the automatic activation of associated evaluations: An overview. Cognition and Emotion, 15: 115-141.

Fazio, R H (1990). A practical givide to the use of response latency in social psychological research in M S. Clark \& C. Hendrick (Eds), Research methods in personailty and social psychology (Pp. 74-97). Thousand Oaks, CA, US: Sage Publications. 
Fazlo, R.H (1986). How do attitudes guide behavior? In R M Sorrentino \& E T Higgins (Eds). Handbook of Cognition and Motivation Foundations of Social Behavior (pp 204-243). New York Guilford Press.

Faivio, R. H, \& Zanna, M. P. (1981). Direct experience and attítude - behavior consistency. In L Berkowitz (Ed). Advances in Experimental Social Psychology (Vol 14, pp. 161-202). New York Academic Press.

Feather, N. T. (Ed). (1982) Expectations and Actions: Expectancy Value Models in Fsychology. Hillsdale, NJ Erlbaum

Feather, N. T. Nomman, M A, \& Worsley, A (1998). Values and Valences: Variables Relating to the Attractiveness and Choilce of Food in Different Contexts Joumal of Applied Social Psychology, 28: $639-656$.

Feldman Barret, L, Gross, J. J., Conner Chnistensen, T., \& Benvenuto, M (2001) Knowing what you're feeling and knowing what to do about it Mapping the relation between emotion differentiation and emotion regulation. Cognition and Emotion, 15: 713-724.

Ferguson, M J, \& Bargh, J. A. (2003). The constructive nature of automatic evaluation. In J. Musch \& K.C. Klauer (Eds), The psychology of evaluation Affective processes in cognition and emotion (pp: 169 188). N. Edbaum

Fishbein, M \& Ajzen, I. (1975). Belief, Attitude, Intention, and Behavior: An Introduction to Theory and Research Reading, MA:Addison-Wesley.

Forgas, I P. (1995). Mood and judgment: The affect infusion model (AIM). Psychological Bulletin, 117: 39 66.

Frank; R. (2004): What price the moral high ground? Princeton, NJ PrincetonUniversity Press.

Fredrickson, B. L (2001). The role of positive emotions in positive psychology. American Psychologist, 56 : 218-226.

Frijda, N. H. (2000). The psychologists' point of view. In M Lewis \& J. M Havilandi-Jones (Eds), Handbook of emations (pp. 59-74). New York Guilford Press.

Frijda, N.H. (1986). The emotions. Cambridge, England Cambridge University Press.

Frijda, N. H., Manstead, A. S. R, \& Bem, S. (2000). The influence of emotions an beliefs. In N. H Frijda, A. S. R. Manstead \& S. Bem (Eds), Emations and Beliefs. How Feelings Influence Thoughts (pp. 1-9). Cambridge: Cambridge University Press:

Frijdă, N. H., \& Mesquita, B. (2000). Beliefs trough Emotions In A s. R Manstead, N. H Finja \& S. Bem (Eds.), Emotions and Beliefs: How Feelings Influence Thoughts (pp. 45-77), Cambridge: Camridge University Press.

Fritzsche, B. A. Finkelstein, M A, \& Penner, L A (2000), To help or not to help: Capturing individuals" decision policies Social Behavior and Personality, 28,561-578.

Gault, B. A, \& Sabini, J (2000). The roles of empathy, anger, and gender in predicting attitudes toward punitive, reparative, and preventative public policies. Cognition and Emotion, 14 495-520.

Gibbons, F. X, Gerrard, M, Blanton, H, \& Russell, D. W. (1998). Reasoned action and social reaction: Willingness and intention as independent predictors of health risk Joumal of Personality and Social Psychology 74: 1164-1180.

Gifford, A.J. (2002). Fmotion and self-control Joumal of Economic Behavior \& Organization, 49:113-130. Gilbert, D. T. (1991). How mental systems believe. American Psychologist, 46; 107-119.

Giner-Sorolla, R. (2001). Guilty pleasures and grim necessities: Affective attitudes in dilemmas of selfcontrol Joumal of Personality and Social Psychology, 80: 206-221 
Godin G, \& Kok, G. (1996). The theory of planned behavior: A review of its applications to healthrelated behavions. Amerian Joumal of Health Promotion, 118798

Godsil, B. P. Tinsley, M R, \& Fanselow, M S (2003) Motivation In A F Healy \& R W. Proctor (Eds.), Handbook of psychology. Experimental psychalogy ( Vol 4 pp. 33-60) New York John Wiley \& Sons.

Gohm, C. L (2003). Mood regulation and emotional intelligence: Individual differences loumal of Personality and Social Psycholagy, 84:594607.

Goldstein, A, Wall, A.M, Mckee, S. A, \& Hinson, R.E (2004). Accessibility of alcohol expectancies from memory: Impact of mood and motives in college student drinkers. Joumal of Studies on Alcohol, 65:95-104.

Gollwitzer, P. M. (1999). Implementation intentions: Strong effects of simple plans. American Psychologist, 54: 493-503.

Gollwitzer, P. M, Heckhausen, H. \& Ratajczak, H. (1990). From weighing to willing: Approaching a change decision trough pre- or postdecisional mentation Organizational Behavior and Human Decision Processes, 45: 41-65.

Gollwitzer, P. M, \& Moskowitz, G. B (1996). Goal effects on action and cognition In E. T. Huggins \& A. W. Kruglanski (Eds) S Social psychology handbook of basic principles (pp. 361-399). NY: Guilford.

Gould, S. I. (2002). Evolution. The pleasures of pluralism. Retrieved January 2, from http://cogwebuclaedu/Debate/Gould htm

Green, L W. \& Kreuter, M W. (1991). Health Promotion Planning: An Educational and Environimental Approach Mountain View, CA Mayfield.

Greene, 1. D, Sommerville, R B., Nystrom, L E, Darley, J M, \& Cohen, J. D. (2001). An AMRI investigation of emotional engagement in moral judgment. Science, 293: 2105-2108.

Greve, W. (2001). Traps and gaps in action explanation: Theoretical problems of a psychology of human action. Psychological Review, 108:435-451.

Gross, J. J. (1998). The emerging field of emotion regulation: An integrative review Review of General Psychology 2:271-299.

Gross, J. J., \& John, O. P. (2003). Individual differences in two emotion regulation processes: Implications for affect, relationships, and well-being loumal of Personality and Social Psychology, 85: 348-362.

Haddock, G. \& Zanua, M. P. (1999). Cognition, affect, and the prediction of social attitudes. In W. Stroebe \& M. Hewstone (Eds), European Review of Social Psychology (Vol. 10, pp. 75-99). London: John Wiley:

Haddock, G, \& Zanna, M P. (1998). Assessing the impact of affective and cognitive information in predicting attitudes toward capital punishment Law and Human Behavior, 22:325-339.

Hagger, M S, Chatzisarantis, N. L D, \& Biddle, S. J. H (2002). The influence of autonomous and controlling motives on physical activity intentions within the theory of planned behavior. British Joumal of Health Psychology, 7: $283-297$.

Hanoch, Y. (2002) "Neither an angel nor an ant": Emotion as an aid to bounded rationality. Joumal of Economic Psychology, 23: 1-25.

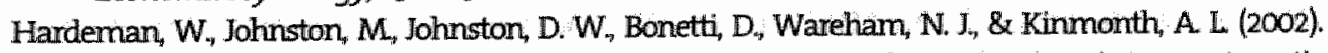
Application of the theory of planned behaviour in behaviour change interventions: a systematic review. Fsychology and Heatth, 17:123-158.

Hertel, G., Neuhof, I, Theuer, T, \& Ken, N. I (2000). Mood effects on cooperation in small groupsi: Does positive mood simply lead to more cooperation? Cognition and Emotion, 14:441-472. 
Hewstone, $M$, \& Young; L. (1988). Expectancy-value models of attitude: Measurement and: combination of evaluations and beliefs. Joumal of Applied Social Psychology, 18: 958-971.

Higgins, E. T. (1997). Beyond pleasure and pain. American Psychologist, 52: 1280-1300.

Higgins, E. T. (1996). Knowledge activation: Accessibility, applicability, and salience In E. T. Higgins \& A. W. Kruglanski (Eds.), Social psychology: Handbook of basic principles. (pp. 133-168). New YorkGuilford Press:

Hinde, R. A (1995). The adaptationist approach has limits Psychological Inquiry, 6:50-53.

Hogan, J. A (1997). Energy Models of Motivation: A Reconsideration. Applied Animal Behaviour Science, 53:89-105.

Holland, R W, Verplanken, B, \& van Knippenberg, A (2002). On the nature of attitude-behavior relations: the strong guide, the weak follow. European Joumal of Social Psychology, 32: 869-876.

Howland, C. L, Janis, L L, \& Kelley, H H (1953), Commumication and Persuasion. New Haven, CT: Yale University Press.

Hufford, M R (2001). An examination of mood effects on positive alcohol expectancies among undergraduate drinkers. Cognition and Emotion, 15: 593-613.

Hume, D. (1739 - 40/1978). A Treatise of Human Nature. LA: Selby-Bigge. Oxford University Press.

Innes-Ker, A, \& Niedenthal, P. M (2002). Emotion concepts and emotional states in social judgement ant categonization. Joumal of Personality and Social Psychology, 83: 804-816.

Izard, C. E. (1993). Four Systerns for Emotion Activation: Cognitive and Noncognitive Processes. Psychological Review, 100:68-90.

lzard, C. E., \& Ackerman, B. P. (2000). Motivational, Organizational, and Regulatory Functions of Discrete Emotions. In M Lewis \& J. M Haviland-Jones (Eds.), Handbook of Emotions (Vol 2, pp. 253-264). NY: Guilford Press.

Izard, C. E, Liberc, D. Z, Putnam, P., \& Haymes, O. M. (1993). Stability of Emotion Experiences and Their Relations to Traits of Personality. Joumal of Pensonality and Social Psychology, 64:847-860.

James, W. (1884). What is an emotion? Mind, 9:188-205.

$\mathrm{Janz}_{i} \mathrm{~N}_{\mathrm{r}}$ \& Becker, M.H (1984). The health belief model: A decade later. Health Eduoation Quarterly, 11 . 1-47.

Johnson, E. J. \& Tversky; A (1983). Affect, generalization, and the perception of risk Joumal of Personality and Social Psychology, $45: 20-31$

Johnson-Laird, P. N, \& Oatley, K. (2000). Cognitive and Social Construction in Emotions. In M. Lewis \& J. M. Haviland-Jones (Eds.), Handbook of Emotions (2 ed, pp. 458-475). New York: Gualford Press.

Johnson-Laird, P. N, \& Qatley, K (1992). Basic Emotions, Rationality; and Folk Theory, Cognition and Emotion, 6: 201-223.

Judd, C.M. McClelland, G.H., \& Culhane, S.E. (1995). Data analysis: Continuing issues in the everyday analyaia of psychological data. Annual Reviews of Psychology, 46:433-465.

Judge, T. A, Erez, A, Bono, J. E, \& Thoresen, C. J. (2002). Are measures of selfesteem, neuroticism, locus of control, and generalized self-efficacy indicators of a comumon core construct? Joumal of Personality and Social Psychology, 83: 693-710.

Julka, D. L $L_{\text {, }}$ \& Marsh, K. L (2000). Matching Persuasive Messages to Experimentally Induced Needs. Current Research in Social Psychology, 5: 1-16.

Kahneman, D. (2003). Mapping bounded rationality. American Psychologist; 58:697-720.

Kamiol, $R_{\psi}$ \& Ross, M (1996). The motivational impact of thinking about the future and past. Annual Revieus of Psychology, 47: 593-620. 
Karoly. $P$ (1993). Mechanisms of self-regulation A systems view. Annwal Review of Pyohology 4423 52.

Katz, D. (1960). The functional approach to the study of attitudes Public Opinion Quarterly, $24: 163-204$

Keltner, D, \& Gross, J.J. (1999). Functional Accounts of Emotions, Cognition and Emotion, 13, 467-480.

Ketelaar, T, \& Au, W. T. (2003). The effects of feelings of guilt on the behavior of uncooperative individuals in repeated social bargaining games: an affect-as-information interpretation of the role of emotion in social interaction Cognition and Emotion, 17:429-453.

Klauer, K C (1998). Affective Priming European Review of Social Psychology, 8.67-103.

Kleinginna, P. R, \& Kleinginna, A. M (1981) A Categrorized List of Emotion Definitions, with Suggestions for a Consensual Definution. Motivation and Emotion, 5: 345-379.

Kok, G., Schaalma, H., De Vnies, H., Parcel, G, \& Paulussen, T. (1996). Social psychology and health education In W. Stroebe \& M Hewstone (Eds.), European Review of Social Psychology (Vol: $7 \mathrm{pp}$. 241-282): John Wiley \& Sons.

Kok, G., van den Bome; B, \& Dolan Mullen, P. (1997). Effectiveness of healtheducation and health promotion: Meta-amalyses of effect studies and determinants of effectiveness. Patient Education and Counseling, 30: 19-27.

Kramer, R M, MoClintock, C. G, \& Messinck, D. M (1986). Social values and cooperative response to a simulated resource conservation crisis. Joumal of Personality, 54, 101-117.

Kristiansen, C. M (1985). Value comelates of preventive health behavior Joumal of Personality and Social Psychology, 49: 748-758.

Kruglanski, A W. (2001). That "vision thing": the state of theory in social and personality psychology at the edge of the new millenium Joumal of Personality and Social Psychology, 80. 871-875.

Kruglanski, A W. (1999). Motivation, cognition, and reality: Three memos for the next generation of research Psychological Inquiny, 10: 54-58.

Kruglanski, A W. (1996). Motivated social cognition Principles of the interface In E T Higgins \& A. W. Kruglanski (Eds.), Social Psychology Handbook of basic principles (pp. 493-520). New York: Guilford Press.

Kruglanski, A. W., Shah, J. Y., Fishbach, A. Friedman, R, Chun, W. Y, \& Sleeth-Keppler, D. (2002). A theory of goal systems. In M P. Zanna (Ed), Advanoes in experimental social psychology(Vol 34, pp. 331 378). San Diego: Acaderric Press.

Kuhl, J, \& Beckmann, J. (1994). Volition and Personality. Göttingen Hogrefe.

Larsen, R J, \& Frednckson, B. L (1999). Measurement Issues in Ernotion Research. In D. Kahmeman, E. Diener \& N. Schwartz (Eds), Well-Being: The foundations of hedonic psychology (pp. 40-60). New York Russell Sage Foundation.

Larsen, R. I, \& Ketelaar, T. (1991). Personality and Susceptibility to Positive and Negative Einotional States. Joumal of Personality and Social Psychology, 61: 132-140.

Lazanis, R. S. (1991). Emotion and adaptation New York: Oxford University Press.

Lazanus, R. S. \& Folkman, S. (1984). Stress, appraisal, and coping. New York: Springer.

LeDoux, J. (1996). The Emotional Brain: The mysterious underpinnings of emotional life. New York: Touchstone.

Lemerise, E. $A_{m} \&$ Arsenio, W. E. (2000). An Integrated Model of Emotion Processes and Cognition in Social Infommation Processing Child Development, 71: 107-118.

Lemer, J. S. \& Keltner, D. (2001). Fear, anger, and nisk Joumal of Personality and Social Psychology, 81 : $146-159$. 
Lemer, I, $S_{*}$ \& Keltmer, D. (2000). Beyond Valence: Toward a Model of Emotion Specific Influences on hudgement and Choice Cognition and Emotion, 14:473-493.

Levesque, $C$, \& Pelletier, L. G (2003). On the investigation of primed and chronic autonomous and heteronomous motivational onientations. Personality and Social Psychology Bulletin, 29: 15701584.

Lewin, $\mathrm{K}$ (1935). Adymamic Theory of Personality: NY McGraw Hill

Lewis, M (2000). Self-Conscious Emotions. Embarrassment, Pride, Shame, and Guilt In M Iewis \& J.M Haviland-Jones (Eds), Handbook of Emotions (pp, 623-636). NY: Guiford Press.

Liebrand, W. B. G, Janssen, R. W. T. L, Rijken, V. M, \& Suhre, C. I. M (1986). Might over morality: social values and the perception of other players in experimental games. Joumal of Experimental Social Psychology, 22: 203-215.

Linnaeus, C (1756) Systema Naturae. Ninth edition. Theodor Haak, Leiden

Locke, E. A, \& Latham, G. P. (1990). A Theory of Goal Setting and Task Performance. Englewood Cliffs, N. Prentice Hall

Loewenstein, G. F, Weber, E. U., Hsee, C. K, \& Welch, N. (2001). Risk as feelings. Psychological Bulletin, 127: 267-286,

Lorenz, K (1937). Uber die bildung des instinktbegniffes. Natunwissenschaften, 25: 289-300.

Lowe, R, Bennett, $P$, Walker, I, Milne, S, \& Bozionelos, G. (2003). A connectionist implementation of the theory of planned behavior: Association of beliefs with exercise intention. Health Psychology, 22 . $464-470$.

Maclean, P. D. (1990). The Trune Brain in Evolution Role in Paleocerebral Functions. Plenum.

Maio, G. R, \& Olson, J. M (2000a). Emergent themes and potential approaches to attitude function: "The function-structure model of attitudes. in G. R Maio \& J. M Olson (Eds.), Why we evaluate: Functions of attitudes (pp. 417442). London: Lawrence Erlbaum.

Maio, G.R., \& Olson, J.M (2000b). What is a value expressive attitude? In G. R. Maio \& J.M. Olson (Eds.), Why we evaluate Functions of attitudes (pp: 249-269). London: Lawrence Erlbaum.

Maio, G. R, \& Olson, J.M. (Eds). (2000c). Why we evaluate: Functions of attitudes. Mahwah, NJ: Lawrence Erlbaum Associates:

Maio, G. R, \& Olson, I. M (1995). Relations between values, attitudes, and behavioral intentions: The moderating role of attitude function. Joumal of Experimental Social Psychology, 31: 266-285.

Manstead A. S. R, \& Parker, D. (1995). Evaluating and extending the theory of planned behaviour. Buropean Review of Social Psychology, 6: 69-95.

Manstead, A. S. R, \& van der Pligt, I. (1998). Should we expect more from expectancy-value models of attitude and behaviour. Joumal of Applied Social Psychology, 28:1313-1316.

Manstead, A. S. R, \& van Eekelen, S. A. M (1998). Distinguishing between perceived behavioral control and self efficacy in the domain of academic intentions and behaviors. Joumal of Applied Social Psychology, 28: 1375-1392.

Markman, A. B. (1999). Knowledge representation. Mahwah, NJ: Lawrence Erlbaum Associates, Inc. Markovsky, B. (1997). Evolution and nebulousmess in theones. Current Research in Social Psychology, 2 24-29.

Maslow, A H (1943). A theory of humanmotivation. Psychological Review, 50: $370-396$.

MoClintock, C. G. (1972). Social motivation - a set of propositions. Behavioral Science, 17:438-344.

MoCullough, M. E., Kilpatrick, S. D. Emmons, R. A. \& Larson, D. B. (2001). Is gratitude a moral affect? Psychological Bulletin, 127: 249-266. 
MicDougall W. (1908). An introduction to social psychology London: Methuen:

MoGurre, W. I. (1969). The nature of attitudes and attitude change In G Lindzey \& E Aronson (Eds) Handbook of Social Psychology (Vol 3, pp, 136-314). Reading, MA: Addison-Wesley.

McKee, S. A, Wall, A-M, Hinson, R.E, Goldstein, A, \& Bissonnette, M (2003) Effects of am implicit mood prime on the accessibility of smaking expectancies in college women Psychology of Addictive Behaviors, 17: 219-225.

Mesquita, B. (2001) Emotions in Collectivist an Individualist Context Joumal of Personality and Social Psychology, 80:68-74.

Millar, M G. \& Millar, K U. (1996). The effects of direct and indirect experience on affective and cognitive responses and the attitude behavior relation Joumal of Expenimental Social Psychology 32: 561-579.

Moll, J., Oliveira-Souza, R. d. Eslinger, P.J, Bramati, L. E., Mourao-Miranda, J., Andreiuolu, P. A, et al, (2002). The neural correlates of moral sensitivity: a functional magnetic resonance imaging investigation of basic and moral emotions. The Joumal of Neurosciences, 22: 2730-2736.

Murphy, G. L, \& Medin, D. L (1985). The role of theories in conceptual coherence Psychological Review 92: 289-316.

Murphy, S. T, \& Zajonc, R B. (1993). Affect, Cognition, and Awareness: Affective Priming with Optimal and Suboptimal Stimulus Exposures. Joumal of Personality and Social Psychology 64: 723-739.

Murray H. A (1938). Explorations in Personality (7 ed). New Xork: Oxford University Press.

Myyry, L, \& Helkema, K (2001). University Students' Value Prionties and Emotional Empathy: Educational Psychology, 21: 25-40.

Naumann, J., \& Richter, T. (2000). Response times in attitudinal items as indicators of the continuous accessibility of knowledge related to attitudes. Psychological Reports, 87:355-366.

Neumann, R. (2000). The Causal Influence of Atributions on Emotions: A Procedural Priming Approach Psychological Science, 11:179-182.

Niedenthal, P. M, Rohmann, A, \& Dalle, N. (2003). What is primed by emotion concepts and emotion words? In J. Musch \& K C. Klauer (Eds.), The psychology of evaluation (pp. 307-333). Mahwah, NJ: Lawrence Erlbaum.

Nisbett, R. E, \& Wilson, T. (1977). Telling more than we can know: Verbal reports on mental processes. Psychological Review, 84. 231-259.

OK Keefe, D. J. (1990). Persuasion: Theoryand Research Newbury Park, CA: Sage Publications.

Oatley, K, \& Jenkins, I.M (1996). Understanding Emotions. Malden: Blackwell Publishers Inc

Ogden I. (2003). Some problems with social cognition models a pragmatic and conceptual andilysis. Heath Psychology, 22: 424-428.

Olson, J M, Roese, N. I, \& Zanna, M P (1996). Expectancies. In E. T. Higgins (Ed), Social Psychology: Handbook of basic principles (pp. 211-238). New York Guilford Press.

Oostveen, T. Kribbe, R, \& De Vries, H. (1996). Social influences on young adults" allohol consumption: norms, modeling, pressure, socializing, and conformity. Addictive Behaviors, 21: 187-197.

Ouellette, J. A. \& Wood, W: (1998). Habit and intention in everyday life: The multiple processes by which past behavior predicts future behavior. Psychological Bulletin, 124: 54-74.

Panksepp, J. (2000a). Emotions as Natural Kinds within the Marnmalian Brain in M Lewis \& J. M Haviland-Jones (Eds), Handbook of Emotions (pp. 137-156). NY: Guilford Press.

Panksepp, 1. (2000b). The neuro-volutionary cusp between ernotions and cognitions. Consciousness \& Emation, 1:15-54. 
Perugini, M. \& Bagozai, R P. (2001). The role of desires and anticipated emotions in goal-directed behaviours Broadening and deepening the theory of planined behaviour. British Joumal of Social Psychology, 40:79.98.

Pervin, L A (1989). Rersons, situations, interactions, the history of a controversy and a discussion of theoretical models. Academy of Management Review, 14:350-360.

Petty, R. A, \& Wegener, D. T. (1998), Attitude change: Multiple roles for persuasion variables. In D. T. Gilbert, S. T. Fiske, \& D. Lindzey (Eds). Handbook of Social Psychology (Vol 1, Pp. 323-39o). Boston: MoGraw-Hili.

Pittiman, T. S. (1998), Motivation In D. Gilbert, S. T. Fiske \& G. Lindzey (Eds), The Handbook of Social Psychology (4ed, Vol 1, pp, 549-590). NY: OxfardUniversity Press.

Ploog, D. (1989). Human Neuroethology of Emotion Neuro-Psychophamacology \& Biological Psychiaty, 13, 15-22.

Plutchik, R. (1991). Emotions and Evolution In K. T. Strongman (Ed), Intemational Review of Studies on Emotion (Vol 1). Sussex Wiley, I \& Sons.

Pooley, I. A, \& O'Connor, M (2000). Environmerital education and attitudes: Emotions and beliefs are what is needed. Environment and Behaviour, 32: 711-723.

Prochaska, J. O, DiClemente, C. C, \& Norcross, J, C (1992). In search of how people change American Psychologist, 47:1102-1114.

Rapoport, A, \& Eshed, L D (1989). Provision of step-level public goods effects of greed and fear of being gypped Organizational Behavior and Human Decision Processes, 44: 325-344.

Redding, C. A., Rossi, J. S., Rossi, S. R, Velicer, W. F., \& Prochaska, J. O. (2000). Health behavior models. The International Electronic loumal of Health Education, 3: 180-193.

Reiss, S. \& Havercamp, S. M (1998). Toward a Comprehensive Assessment of Fundamental Motivation: Factor Structure of the Reiss Profiles. Psychological Assessment, 10:97-106.

Rhodes, R. E., Coumeya, K S, \& Jones, L W. (2004). Personality and social cognitive influences on exercise behavior adding the activity trait to the theory of planned behavior. Psychology of Sport and Exercise, $5: 243-254$.

Richard, R., de Vries, N. K., \& van der Pligt, J. (1998). Anticipated regret and precautionary sexual behavion. Joumal of Applied Social Psychology, 28: 1411-1428.

Richard, $R_{*}$ van der Pligt, $\mathrm{I}_{*}$ \& de Vries, $\mathrm{N}_{\mathrm{N}} \mathrm{K}$. (1996). Anticipated affect and behavioral choice. Basic and Applied Social Psychology, 18: 111129.

Robinson, M. D. \& Clore, G. L (2002). Belief and feeling: Evidence for an accessibility model of emotional self-report Psychological Bulletin, 128:934960.

Rogers, R. W. (1983). Cognitive and psychological processes in fear appeals and attitude change: A revised theory of protection motivation. In J. T: Cacioppo and R E. Petty (Eds.), Social Psychophysiology (pp. 153-176). NY: Guilford.

Rokeach, M (1973). The Nature of Human Values. New York: Free Press.

Rose, H. \&x Rose, S. (Eds). (2000). Alas poor Danwin London: Jonathan Cape.

Roseman, I. J., Wiest, C, \& Swartz, T. S. (1994). Phenomenology, Behaviours, and Goals Differentiate Discrete Emotions Joumal of Personality and Social Psychology, 67: 206-221.

Rosenberg, M I: (1956). Cognitive structure and attitudinal affect: Joumal of Abnomal and Social Psychology, 53: 367-372.

Rotter, I. B. (1966). Generalized expectancies for intemal versus extemal control of reinforcement. Psychological Monographs (General \& Applied), 80: 1-28. 
Rozin, P, \& Cohen, A. B. (2003). High frequency of facial expressions comesponding to confusion. concentration, and worry in an analysis of naturally occurring facial expressions of Americans. Emation, 3, 68-75.

Rozin, P. Haidt, I, \& MoCauley, C.R. (2000) Disgust In M Lewis, \& 1 M Haviland-Jones (Eds.), Handbook of Emotions (Pp. 137-156). New York Guilford:

Ryan, R M, \& Deci, E L (2000) Self-Determination theory and the facilitation of intrinsic motivation, social development, and weil-being American Psychologist, 55:68-78.

Sallis, J. F, Bauman, A, \& Pratt, M (1998), Environmental and policy interventions to pronote physical activity. Amenioun Ioumal of Preventive Medicine, 15: 379-397.

Scherer, K R (1999). Appraisal theory In T Dalgleish \& M Power (Eds), Handbook of Cognition and Emotion (pp 637-663). Chichester, England Wiley.

Schmidt, F. L (1973). Implications of a measurement problem for expectancy theory research. Organizational Behavior and Human Performance, 10:243-251

Schwartz, S. H. (1994). Are there universal aspects in the structure and contents of human values? Joumal of Social Issues, 50: 19-45.

Schwartz, S. H. (1992). Universals in the structure and content of values: Theoretical advances and empinical tests in 20 countries. Advances in Experimental Social Psychology, 25; 1-65.

Schwarz, N. (2000). Emotion, Cognition, and decision making. Cognition and Emotion, 14 433-440.

Schwarz, N. (1999). Self-reports: how the questions shape the answers. American Psychologist, 54: 93105.

Schwar, N., \& Clore, G. L (1996). Feelings and phenomenal experiences. In E. T. Higgins \& A. W. Kruglanski (Eds), Social Psychology. Handbook of Basic Principles (pp. 433-465). NY: Guilford.

Shackelford, T. K. (2000). Emotional Reactions to Infidelity. Cogmition and Emotion, 14 643-659.

Shafir, E, \& LeBoeuf, R. A (2002). Rationality. Annual Review' of Psychology, 53: 491-517.

Sheeran, P. (2002). Intention-Behavior relations: A conceptual and empirical review. In W. Strocebe \& $M$

Hewstone (Eds.), European Review of Social Psychology (Vol 12, pp. 1-36). West Sussex, England: John Wiley.

Sheeran, P., \& Abraham, C. (2003). Mediator of moderators: Temporal stability of intention and the intention-behavior relation Personality and Social Psychology Bulletin, 29: 205-215.

Sheeran P. Conner, M, \& Noman, P. (2001) Can the theory of planned behavior explain pattems of health behavior change? Health Psychology, 20: 12-19.

Sheldon, K M, Elliot, A, I. Kin, $\mathrm{Y}_{*}$ \& Kasser, T. (2001). What is Satisfying about Satisfying Events? Testing 10 candidate psychological needs. Joumal of Personality and Social Psychology, 80: 325339.

Sheppard, B. H. Hartwick, J. \& Warshaw, P. R (1988). The theory of reasoned action A meta-analysis of past research with recommendations for modifications and future research Joumal of Consumer Research, 15: 325-343.

Sideridis, G. D., \& Kaissidis-Rodafinos, A. (2001). Goal importance within planned behaviour theory as the' predictor of study behaviour in college British Joumal of Educational Psychology, 71: 595618.

Smith, C. A, \& Ellsworth, P. C (1985) Pattems of Cognitive Appraisal in Emotion Joumal of Personality and Social Psychology, 48: 813-838.

Smith, IL (1999). An agentic psychology model based on the the paradigmatic repositioning of the theory of planned behavior. Theory \& Psychology, 9:679-700. 
Smuth, IL (1996). Expectancy, value, and attitudinal semantics. European loumal of Social Psychology, 26. $501-506$.

Sinith, M B. Bruner, J.S, \& White, R W. (1956). Opinions and Personality New York. Wiley.

Sryder M, \& DeBono, K G. (1985). Appeals to image and claims about equality: Understanding the psychology of advertising Joumal of Personality and Social Psychology, 49: 586-597.

Solomon, M R (2002). Consumer Behavior Buying, Having; and Being. Upper Saddle River, NI: Prentice Hall.

Sparis, P. \& Guthrie, C A (1998). Self-identity and the theory of planned behavior A useful addition or an whelpful artifice? Joumal of Applied Social Psychology, 28: 1393-1410.

Sparks, P, Hedderley, D, \& Shepherd, R (1991). Expectancy-Value models of attitudes: A note on the relationship between theory and methodology. European Joumal of Social Psychology, 21: 261271.

Spencer, S. J., Fein, S, Wolfe, C. T. Fong, C, \& Dimn, M A (1998). Automatic activation of stereotypes: The role of self-image threat Personality and Social Psychology Bulletin, 24:1139-1152.

Srull, T. K, \& Wyen, R. S, Ir. (1979). The role of category accessibility in the interpretation of information about persons: Some determinants and implications. Joumal of Personality and Social Psychology, $37: 16601672$

Stanley, K D, \&. Murphy, M R (1997). A Comparison of General Self-Efficacy with Self-Esteem. Genetic, Social, and General Psychology Monographs, 123 .79-99.

Stein, N. L \& Oatley, K (1992). Basic Emotion: Theory and Measurement Cognition and Emotion, 6: 161-168.

Strack, F, Schwarz, H, \& Gschneidinger, E (1985). Happiness and reminiscing: the role of time perspective, mood, and mode of thinking loumal of Personality and Social Psychology, 49; 1460 1469.

Strahan, E. J Spencer, 5 J, \& Zanma, M P. (2002). Subliminal priming and persuasion: Striking while the iron is hot joumal of Experimental Social Psychology, 38: 556-568.

Sutton, S. (2002a). Testing attitude-behavior theories using non-experimental data: An examination of some hidden assumptions. In W. Stroebe \& M Hewstone (Eds), European Review of Social PSychology (Vol 13, pp 299-323). East Sussex, England: Psychology Press:

Sutton, S: (2002b). Using social cognition models to develop health behaviour interventions: Problems and Assumptions. In L. Quine \& D. Rutter (Eds), Changing health behaviour: Intervention and research with socal cognition models (pp. 193-208). Buckingham, England: Open University Press.

Sutton, S: (1998). Fredicting and explaining intentions and behavior How well are we doing? Joumal of Applied Social Psychology, 28: 1317=1338.

Swanborn, P. G. (1996). Wetenschappelijke ontwikkelinger: De Fishbein/Ajzentheorie in de kritiek Nederlands Tydschift voor de Psychologie, 51:35-46.

Tangney. J. P. (1990). Assessing individual differences in proneness to sharne and guilt development of the self-conscious affect and attribution inventory. Ioumal of Personality and Social Psychology, 59: 102-111.

Toates, F. M (1998). The Interaction of Cognitive and Stimulus Response Processes in the Contral of Behaviour. Neuroscience and Biobehavioral Revieus; 22:5983.

Tomkins, S. S (1962). Affect, imagery, and consciousness: Vol 1. The positive affects. New York. Springer.

Tooby, I, \& Casmides; L (1990). The Past Explains the Present: Emotional Adaptations and the Structure of Ancestral Environments. Ethology and Sociobiology, 11: 375-424. 
Trafmow, D, \& Finlay, K A (2002). The prediction of attitudes from beliefs and evaluations: the logic of the double negative. British Joumal of Social Psychology, 41: 77-86.

Trafimow, D, Sheeran, $\mathbb{P}_{\text {, Conner }}$ M, \& Finlay, K A (2002). Evidence that perceived behavioural control is a multidimensional constnuct: perceived control and percerved difficulty. British Joumal of Social Psychology, 41:101-121.

Trafimow, D., Sheeran, P. Lombardo, B. Finlay, K A, Brown, J, \& Armitage; C J. (2004). Affective and cognitive contral of persons and behaviours. British Joumal of Social Pyychology, 43: 207-224.

Thivers, R L (1971). The evolution of reciprocal altwism Quarterly Review of Biology, 46:35-57.

Vallacher, R.R, \& Wegner, D M (1985). A Theory of Action Identification Hillsdale, N. Erlbaum.

Vancouver, J. B. \& Scherbaum C. A. Jr. (2000). Automaticity, goals, and envirommental interactions. American Psychologist, 55:763-764.

Van den Putte, B. (1991). 20 years of the theory of reasoned action of Fishbein and Ajzen: A metaanalysis. Unpublished manuscript, University of Amsterdam.

Van der Pligt, J, \& de Vries, N. K (1998a). Belief importance in expectancy-value models of attitudes. Joumal of Applied Social Psychology, 28: 1339-1354

Van der Pligt, J, \& de Vries, N. K (1998b). Expectancy-Vahue Models of Health Behaviour: The Role of Salience and Anticipated Affect. Psychology and Health, 13: 289-305.

Van der Pligt, J., de Vries, N. K, Manstead, A. S. R, \& van Harreveld, F (2000). The importance of being selective: Weighing the role of attribute importance in attitudinal judgement. Advances in Experimental Social Psychology, 32:135-200.

Van der Pligt, $\mathrm{J}_{4}$ \& Eiser, J. R. (1984). Dimensional salience, judgment, and attitudes. in J. R. Eiser (Ed.), Attitudinal Judgment (pp, 43-63). NY, Springer-Verlag.

Van der Pligt, J, Zeelenberg, M, van Dijk, W. W, de Vries, N. K, \& Richard, R. (1998). Affect, attitudes and decisions: Let's be more specific In W. Stroebe \& M. Hewstone (Eds.), European Review of Social Psychology (Vol 8, pp. 33-66). NY: John Willey.

Van Harreveld, F, van der Pligt, J, de Vries, N. K, \& Andreas, S. (2000). The structure of attitudes: attribute importance, accessibility and judgement. British Journal of Social Psychology, 39: 363380 .

Van Lange, P. A M (2000). Beyond self-interest: a set of propositions relevant to interpersonal onentations. In M Hewstone \& W. Stroebe (Eds.), European Review of Social Psychology, 11, (pp. 297-331). London: Willey.

Van Lange, P. A M (1999). The pursuit of joint outcomes and equality in outcomes: an integrative model of social value onientation. Joumal of Personality and Social Psychology, 77:337-349.

Van Lange, P. A. M, Otten, W., De Bruin, E. N. M, \& Joireman, J. A. (1997). Development of prosocial, individualistic, and competative onientations: theory and preliminary evidence Joumal of Personality and Social Psychology, 73, 733-746.

Verplanken, B., \& Aarts, H. (1999). Habit, attitude, and planned behavior: Is habit an empty construct or an interesting case of goal-directed automaticity? In W. Stroebe \& M. Hewstone (Eds.), European Review of Social Psychology (Vol 10, pp. 101-134). NY: John Wiley.

Verplanken, B., Hofstee, G. \& Janssen, H. J. W. (1998). Accessibility of affective versus cognitive compoments of attitudes. European Ioumal of Social Psychology, 28: 23-35.

Weddell, R. A. (1994). Effects of Subcortical Lesion Site on Human Emotional Behaviour. Brain and Cogrition, 25: 161-193. 
Weinstein, N. D. (1993). Testing four competing theonies of health-protective behaviour. Health Psychology, 12: 324-333.

Weinstein, N. D, Rothman, A J.s Sutton, S. R (1998). Stage theories of health behavior: conceptual and methodological issues. Health Psychology, 17: 290-299.

Wicker, A. W. (1969): Attitude versus action: The relationship of verbal and overt behavioural responses to attitude objects loumal of Social lssues, $25,41-78$.

Zajonc. R. B. (1998). Emotions In D. Gilbent, S. T. Fiske \& G. Lindzey (Eds), The handbook of social psycholagy (pp. 591 632). NY: Oxford University Press.

Zajonc, R. B. (1980) Feeling and thinking: Preferences need no inferences. American Psychologist, 35 : 151-175.

Zajonc, R B. (1968). Attitudinal effects of mere exposure. Joumal of Personality and Social Psychology, 9 : 1-27.

Zeelenberg, M, \& Pieters, R (2004). Beyond valence in customer satisfaction: A review and new findings on behavioral responses to regret and disappointment in failed services. Joumal of Business Research, 57: 445-455.

Zeelenberg, M, van Dijk, W. W., Manstead, A. S. R, \& van der Pligt, J. (2000). On bad decisions and disconfirmed expectancies: The psychology of regret and disappointment Cognition and Emotion, 14:521-541. 


\section{Summary}

This dissertation presents an attempt to integrate two, general accounts of behavior: the Reasoned Action and the Motivational perspective.

As outlined in Chapter 1, the Reasoned Action perspective has dominated behavioral theories in the fields of social- and health-psychology for the better part of the previous century. Formalized in Expectancy-Value models, Reasoned Action accounts consider behavior to be the product of intentional choice, which is ultimately based on the deliberation of perceived advantages and disadvantages of following a certain course of action Accordingly, health education interventions that are grounded in this theoretical perspective hold that behavioral change can be achieved through modification of people's expectancies regarding the consequences of a behavioral decision. We investigated the extent to which this particular approach to health education can be maintained by exploring the psychological validity of Expectancy-Value accounts of behavior. Deficiencies in the description of the psychological processes underlying behavior as provided by ExpectancyValue models, would limit their utility as a framework for theory-based health education.

To evaluate their psychological validity, Chapter 2 presents a comprehensive and structured review of comments to Expectancy-Value models that have accumulated during the past two decades. The first category of comments concems contributions that aim to expand the standard Expectancy-Value model with additional variables in order to improve its predictive potential in terms of the variance explained. Though indicating that current Expectancy-Value models are open to improvement in this domain, this way of model expansion has been criticized for it neglects to determine whether the associations between variables as specified in the model actually represent causal relations. If not, health education cannot be based on such a model for behavior change, for it requires the specification of causal determinants, rather than mere predictors of action.

A second category of contributions indeed focuses on issues of causality. It is argued that Expectancy-Value models fail to provide a satisfactory description of the psychological 
processes mediating between the formation of intention and the execution of behavior and of the processes by which the anticipation of behavioral outcomes results in the elicitation of action Pertaining to the latter, it is stipulated that outcome expectancies, as specified in current EV models, lack motivational potential. That is, nothing about an outcome expectancy, defined as a perceived consequence of acting, necessitates the holder of that belief to act accordingly. In other words, what is lacking from current expectancy-value models is the motivational drive that energizes behavior. Several authors have advocated that to fill this motivational void, Expectancy-Value models should be integrated with goalconstructs. We conclude, however, that attempts in this direction, so far, have been inadequate.

Behavioral explanations in terms of the goals that individuals try to attain, characterize the Motivational perspective on behavior, which is outlined in Chapter 3. Essentially, the Motivational perspective conceives of behavior as the joint product of individual needs or desires and demands and opportunities for their achievement as presented by the environment. We start this chapter by suggesting that a successful integration of Expectancy-Value models with goal-constructs requires the identification of the general motives underlying human behavior. We argue that previous attempts to develop a taxonomy of general goals failed because researchers did not have a clear conception about the origin, and hence, about the nature of these goals. Drawing on evolutionary theory, we propose that the general goals of behavior denive from adaptive problems; recurrent situations presenting opportunities or challenges to the maximization of individual fitness.

We subsequently propose that the spectrum of human emotions may serve as an adequate index of these adaptive problems, and thus, of the goals underlying human behavior in general Empirical research and theoretical arguments from functional accounts of emotions seem to support this proposal. According to functional theories, specific emotions (e.g. fear), specify general goals (e.g, personal safety), and motivate behavioral responses (e.g, nunning away) performed to attain these goals. Studies indeed attest for the goal-related nature of emotions by demonstrating emotion-specific differences in (a) the perception of situational cues eliciting the emotion, (b) the action tendency that ensues from an emotional state, and (c) associated goal-states.

Finally, we present two empincal studies in which we provided further support for our conception of emotions as goals. In Study 3.1 we establish relations between emotions and values, another construct proposed to explicate fundamental goals. The results of this study show that if people consider a particular value (e.g, safety) to be an important guiding principle in their daily lives, they also report more frequent experience of associated emotions (e.g, fear). This indicates that the more people strive towards a certain goal, the more they experience the particular emotion that, according to functional perspectives, constitutes the primary motivational force underling behavior to attain this goal. Moreover, similar relations between values and emotions were found in relation to the performance of specific behaviors. For example, the more people consider having unprotected sex to violate safety values, the more afraid they expect to feel when doing so, which also indicates that the 
attainment or frustration of particular goals is associated with specific emotional responses. In Study 3.2, we show that activating an emotional state influences behavior (in a prisoner dilemma game) only if individuals do not already have a chronically active goal that is congruent (in terms of action tendency) to the temporary activated goal related to the emotional state.

In Chapter 4 we introduce an integrated perspective on behavior, in which outcome expectancies as specified in Expectancy-Value models, are related to the general goals underlying behavior, as specified by the spectrum of human emotions. Hereto, an outcome expectancy is defined as an internal representation that specifies how: performing a particular act is conducive to or frustrates the attainment of a general goal. This integrated perspective holds that, because of their association with general goals, the impact of outcome expectancies on behavior is (at least to some extent) dependent upon the operation of the basic motivational systems (i.e, emotions), directing action to attain these goals. According to the integrated perspective, behavior causally follows from anticipation of its outcomes to the extent that general goals are activated in this deliberation process. The integrated perspective, however, implies yet another way in which intentional, goal-directed action follows from a particular decision situation Specifically, the activation of a goal may affect people's perception of goal-related outcomes as well as the other way around: This in tum, may influence the relation between outcome expectancies and behavior.

It was hypothesized that goal-activation could influence the relation between outcome expectancies and behavior through changes in accessibility or evaluation (either in terms of likelihood, valence, or both) of goal-related outcome expectancies. Additionally, goalactivation may also moderate the relation between outcome expectancies and behavior. We conducted two experiments to test these hypotheses.

In Study 4.1 we activated goals (safety and cooperation) by inducing emotional states (fear and guilt, respectively). We investigated the effects of goal-activation on outcome expectancies related to interfering with random violence that were associated to either the personal safety or the cooperation goal. In Study 4.2 we activated the same goals, but this time by a semantic priming procedure (scrambled sentences). We investigated the influence of goal-activation on the endorsement of information as specified in a message. The message specified two general consequences from the production of a genetically modified banana, one positive consequence related to the cooperation goal, the other, negative, consequence related to the goal of personal safety.

The results provided limited support for the hypothesized consequences of goalactivation. In Study 4.1, goal-activation was found to enhance expectancy-value ratings of associated outcome expectancies, but only to the extent that the particular outcome was not strongly associated to the behavior, as indicated (post-hoc) by participants' response latencies. Similarly, in Study 4.2 , we found that activating the goal of Cooperation increased people's endorsement of consequences that were associated to this goal. Again, this effect orily concerned weakly associated beliefs, as the consequences that were specified in the message were fictional and thus novel to all participants. Finally, the results of Studies 4.1 and 4.2 also 
indicated that goal-activation could directly influence behavior (or intentions), that is, independent from reasoned action processes.

Finally, in Chapter 5, we reflect on the implications of the results from our studies and the integrated perspective in general Pertaining to current Expectancy-Value models, it is argued that the relation between general goals and behavior-specific outcome expectancies as specified by the integrated perspective substantiates the proposed causal influence of anticipating behavioral consequences on action. Though further research is required to determine the way in which goal-activation affects reasoned action processes, we content that eventually, goal-constructs should be introduced in Expectancy-Value models to provide a more accurate representation the psychological processes underlying goal-directed behavior.

Subsequently, pertaining to the effectiveness of information-based health education methods (i.e., persuasion); it is argued that the integrated perspective suggests that the motivational potential of such messages will be higher if they are targeted at individually salient goals for the behavior under investigation. As such, these rellevant goals may constitute a basis for the tailoning of health education programs. On the other hand, the consequences of the integrated perspective on behavioral control may also imply certain limitations for the effectiveness of behavioral interventions applying information-based methods, because: (1) The influence of outcome expectancies on behavior is not constant but dependent upon fluctuations in the motivational state of the individual, hence, even accepted outcomes as specified in a message need not always affect behavioral decisions. Additionally, (2) communicated consequences often lack motivational potential because they are generally held with less certainty than expectancies that are based on direct experience. Finally, (3) goal-activation may influence behavioral decisions independent from and predominant over reasoned action processes.

This direct influence of goal-activation often is the result of behavioral tendencies induced by particular emotions, which are elicited by situational cues As such reactive emotional influences are likely to be a major determinant of unhealthy behaviors. It is argued that research on emotion-regulation processes deserves great attention from researchers in the fields of health-education and - psychology in the years to come. 


\section{Samenvatting}

In dit proefschrift is getracht een integratie te realiseren van twee, algemene perspectieven op gedrag, te weten het Beredeneerde en het Motivationele perspectief.

Hoofdstuk 1 begint met een uiteenzetting van het Beredeneerde perspectief op gedrag, dat gedurende het grootste deel van de vonige eeuw theorieen en modellen op het gebied van sociale, - en gezondheidspsychologie gedomineerd heeft. Het beredeneerde perspectief, geformaliseerd in verwachtingswaarde modellen, stelt gedrag voor als het product van een intentionele keuze, welke uiteindelik gebaseerd is op een afweging van de verwachte voor, - en nadelen van het gedrag in . kwestie. Derhalve gaan gedragsinterventies die gebaseerd zijn op dit perspectief er vanuit dat gedragsverandering bewerkstelligt kan worden door het veranderen van verwachtingen die mensen houden ten aanzien van de consequenties van hun gedragsikeuze. Wij onderzochten in hoeverre deze manier van gezondheidsvoorlichting gerechtvaardigd is ten overstaan van de psychologische validiteit van verwachtingswaarde modellen. Immers, tekortkomingen in de beschrijving van de psychologische processen onderliggend aan gedrag zoals gepresenteerd in deze modellen, zou een beperking betekenen van hun bruikbaarheid als kapstok voor theoriegestuurde gezondheidsvoorlichting.

Teneinde de psychologische validiteit van verwachtingswaarde modellen te evalueren, wordt in Hoofdstuk 2 een uitgebreide, gestructureerde review gepresenteerd van verschillende commentaren ten aanzien van deze modellen die in de voorbije decennia in de literatuur zijn geaccumuleerd. Een eerste categorie van commentaren betreft voorstellen tot uitbreiding van het standaard verwachtingswaarde model met additionele variabelen, teneinde de voorspellende kracht van het model, uitgedrukt in het percentage verklaarde variantie, te vergroten Hoewel deze bijdragen uitwijzen dat er ruimte is voor verbetering op dit gebied, heeft deze aanpak van model verbetering ook knitiek geoogst omdat vaak voorbij gegaan wordt aan de vraag of relaties tussen variabelen ook daadwerkelijk causiale verbanden betreffen.

Een tweede categorie van uitbreidingen concentreert rich dan ook specifiek op de vermeende causale aard van de verbanden tussen variabelen. Er wordt betoogd dat 
verwachtingswaarde modellen geen afdoende beschuijving geven van de psychologische processen die mediëren tussen het wormen van een intentie en het uitvoeren van gedrag en evenmin van de processen waardoor de anticipatie van gedragsuitkomsten zou leiden tot het uitvoeren van gedrag. Met betrekking tot het laatste wordt gesteld dat het uitkomstverachtingen, zoals gespecificeerd in huidige verwachtingswaarde modellen, ontbreekt aan motivationeel potentieel. Hiermee wordt bedoeld dat het hebben van een bepaalde uitkomstverwachting, gedefinieerd als een verwacht gevolg van gedrag, een persoon niet noodzaakt ook dienovereenkomstig te handelen. Met andere woorden, het ontbreekt huidige verwachtingswaarde modellen aan een motivationele component die actie initieert. Verschillende auteurs stellen dat doelconstructen geïntegreerd moeten worden in het huidige verwachtingswaarde model om deze motivationele lacune op te heffen.

Gedragsverklaringen in termen van doelen welke een persoon probeert te realiseren kenmerken het Motivationele perspectief op gedrag. Dit wordt beschreven in Hoofdstuk 3. In wezen stelt het Motivationele perspectief gedrag voor als het gezamenlijke product van individuele wensen en behoeften, alsmede de vereisten en mogelijkheden welke de situatie presenteert tot hun realisatie. We beginnen dit hoofdstuk met de bewering dat een succesvolle integratte van doelen in verwachtingswaarde modellen een identificatie vereist van de algemene doelen die menselijk gedrag onderliggen. We stellen dat vorige pogingen tot identificatie van deze doelen gefaald hebben doordat onderzoekers geen duidelijke notie hadden omtrent de oorsprong en dientengevolge over de aard van deze fundamentele doelen Gebaseerd op evolutietheorie, stellen we dat de algemene doelen onderliggend aan gedrag hun corsprong vinden in adaptieve problemen; herhaaldelijk voorkomende situaties waarin een bepaalde gedragskeuze consequente implicaties had voor de fitness van een individu.

Vervolgens wordt uiteengezet dat het spectrum van menselijke emoties kan fungeren als een index van deze adaptieve problemen en daarmee van de algemene doelen onderliggend aan menselijk gedrag. Er wordt cen overzicht gegeven van theoretische argumenten en empirische gegevens die dit voorstel staven Volgens functionele emotietheoneèn specificeren specifieke emoties (zoals angst) algemene doelen (bijvoorbeeld persoonlijke veiligheid) en motiveren ze gedrag (bijvoorbeeld vluchten) dat erop genicht is deze doelen te realiseren. Deze relatie tussen emoties en doelen wordt bevestigd door studies die wijzen op emotie specifieke verschillen in (a) situatie gerelateerde signalen die de emotie opwekken, (b) de actiebereidheid die ontstaat als gevolg van een bepaalde emotionele toestand, en (c) gerapporteerde associaties met doelen.

Tenslotte worden twee empinische studies gepresenteerd die verdere ondersteuning bieden voor onze conceptie van emoties als doelen In Studie 3.1 tonen we relaties tussen emoties en waarden, een ander construct dat verondersteld wordt fundamentele doelen te specificeren. De resultaten van deze studie suggereren dat indien personen een bepaalde waarde (zoals veiligheid) beschouwen als een belangnijke leidraad in hun dagelijks handelen, ze eveneens rapporteren vaker emoties (zoals angst) te voelen die geassocieerd zjpn met hetzelfde doel. Dit wijst erop dat hoe meer mensen naar een bepaald doel streven, hoe meer ze de emotie ervaren die, volgens het functionele perspectief, het primaire motivationele systeem vormt dat gedrag onderligt dat genicht is op het bereiken van dat doel. Daarnaast werden vergelijkbare relaties 
tussen emoties en waarden ook gevonden in relatie tot het uitvoeren van een specifiek gedrag. Bijpoorbeeld, hoe meer mensen van mening waren dat onveilig vrijen strijdilg was met velligheid waarden, hoe meer ze verwachtten zich angstigg te voelen als ze dat toch zouden doen. Dit wijst erop dat het al dan niet bereiken van een bepaald doel gepaard gaat met specifieke emotionele reacties. In Studie 3.2 vervolgens, wordt aangetoond dat het induceren van een bepaalde emotionele toestand gedragskeuzes beinvloedt, doch enkel indien een persoon niet reeds een chronisch actief doel heeft dat overlapt (in temen van gedragsbereidheid) met het tijdelijk geactiveerde doel als gevolg van de emotionele toestand.

In Hoofdstuk 4 wordt een geintegreerd perspectief op gedrag geintroduceend Hierin zijn uitkomstverwachtingen, zoals beschreven in verwachtingswaarde modellen, gerelateerd aan de algemene doelen van gedrag zoals gespecificeerd door het spectrum van menselijke emoties. Hiertoe zijn uitkomstverwachtingen gedefinieerd als interne representatie over de implicaties van het uitvoeren van een handeling voor het al dan niet bereiken van een bepaald doel Volgens het geintegreerde perspectief is, vanwege hum relatie met algemene doelen, de invloed van uitkomstverwachtingen op gedrag (tot op bepaalde hoogte) afhankelijk van de activatie van de basale motivationele systemen (lees, emoties), welke het gedrag sturen dat erop gericht is deze doelen te realiseren. Aldus wordt gedrag causaal veroorzaakt door anticipatie van de uittkomsten ervan, in zoverre dat deze algemene doelen geactiveerd worden in het afwegingsproces. Volgers het geïntegreerde perspectief is er echter nog een manier waarop intentioneel, doelgericht gedrag optreedt in een bepaalde keuze situatie. Namelijk, doordat de activatie van een doel de persoonlijke verwachting van doelgerelateerde uitkomsten beinvloedt, in plaats van andersom, waardoor ook de relatie tussen uitkomstverwachtingen en gedrag verandert.

Verwacht werd dat doelactivatie de relatie tussen uitkomstverwachtingen en gedrag kan beinvloeden door veranderingen in toegankelijkheid en evaluatie (zowel in termen van waarschijnlijkheid als positieve of negatieve evahuatie) van doelgerelateerde uitkomstverwachtinger. Bovendien zou doelactivatie de relatie tussen gerelateerde uitkomstverwachtingen en gedrag kunnen modereren. Deze verwachtingen zijjn middels twee experimentengetoetst.

In Studie 41 werden doelen (velligheid en cooperatie) geactiveerd door inductie van emotionele toestanden (respectievelijk angst en schuld). We onderzochten de effecten van doelactivatie op uitkomstverwachtingen over fysiek ingrijpen bij zinloös geweld welke gerelateerd waren aan hetzij het veiligheidsdoel, hetzij het coöperatiedoel In Studie 4.2 werden dezelfde doelen geactiveerd, maar dit maal door middel van een semantische prining procedure. We onderzochten de invloed van doelactivatie op de mate waarin mensen bepaalde informatie uit een voorlichtingstekst onderschreven. In deze tekst werden twee algemene consequenties geschetst aangaande de productie van een genetisch gemanipuleerde banaan. Te weten, één positieve consequentie, die relateerde aan het coöperatiedoel en eén negatieve consequentie, gerelateerd aan het veiligheidsdoel.

De resultaten boden weinig ondersteuning voor de verwachte invloeden van doelactivatie In Studie 4.1 vonden we dat doelactivatie het verwachtingswaarde product (gerapporteerde waarschijnlijkheid $x$ evaluatie) van doelgerelateerde uitkomstverwachtingen beinviloede, maar 
enkel indien de uitkomst in kwestie niet reeds sterk geassocieerd was met het gedrag, zoals (achteraf) gemeten aan de hand van individuele verschillen in reactiesnelheid. In Studie 4.2 werd gevonden dat activatie van het cooperatiedoel er inderdaad toe leidde dat mensen de daaraan gerelateerde consequenties in sterkere maten onderschreven. Wederom betrof dit effect enkel zwak geassocieerde uitkomstverwachtingen, daar de in de tekst gespecificeerde consequenties fictief, en dus nieuw voor alle deelnemers waren Zowel de resultaten van Studie 4.1 als van Studie 4.2 tenslotte, suggereerden dat doelactivatie gedrag ook direct kon beïnvloeden, dat wil zeggen, onathankelijk van processen van Beredeneerd gedrag.

Hoofdstuk 5, tenslotte, presenteert een aantal bespiegelingen over de mogelijke implicaties van de gevonden resultaten en van het geintegreerde perspectief in het algemeen. Met betrekking tot verwachtingswaarde modellen wordt betoogd dat de relatie tussen algemene doelen en daaraan gerelateerde uitkornstverwachtingen zoals beschreven in het geintegreerde perspectief, de voorgestelde causaliteit staaft, van de relatie tussen het anticiperen van gedragsconsequenties en het uitvoeren van gedrag. Hoewel verder onderzoek noodzakelijk is om te bepalen op welke manier processen van Beredeneerd gedrag beinvloedt worden door doelactivatie, zijn we ervan overtuigd dat doelconstructen uiteindelijk geïntroduceerd zullen moeten worden in de huidige verwachtingswaarde modellen, teneinde een meer nauwkeurige representatie te ontwikkelen van de psychologische processen waarlangs doelgericht gedrag tot stand komt.

Met betrekking tot de effectiviteit van informatiegeoniënteerde methoden van gezondheidsvoorlichting, wordt vervolgens gesteld dat het geintegreerde perspectief impliceert dat de motivationele impact van dergelijke boodschappen groter wordt indien ze aansluiten bij individueel saillante doelen voor het gedrag in kwestie. Als zodanig kunnen dergelijke relevante doelen een basis bieden voor het ontwikkelen van ... voorlichtingsprogramma's. Aan de andere kant zou het geintegreerde perspectief echter bepaalde beperkingen impliceren voor wat betreft de effectiviteit van gedragsinterventies op basis van informatiegeoriënteerde methoden, en wel omdat: (1) De invloed van verwachtingen op gedrag niet constant is maar afhankelijk van fluctuaties in de motivationele toestand var een individu. Dus zelf indien de consequenties zoals beschreven in een voorlichtingstekst geaccepteerd worden, hoeft dit nog niet te betekenen dat ze ook een consequente invloed uitoefenen op gedragskeuzes. Het bovendien (2), de invloed van gecommuniceerde consequerties veelal ontbreekt aan motivationeel potentieel daar ze in het algemeen met minder zelkerheid onderschreven worden dat consequenties welke gebaseerd zijn op directe ervaringen. Ten slotte (3), de invloed van doelactivatie op gedragskeuzes onafhankelijk kan zijn van, - en in deze gevallen meestal ook dominant is over processen van Beredeneerd gedrag.

Deze directe invloed van doelactivatie is vaak het gevolg van actietendensen die opgewekt worden door bepaalde emotionele toestanden, welke door situationele signalen worden opgeroepen. Omdat dergelijke emotionele invloeden naar alle waarschijnlijkheid een belangrijke determinant van ongezond gedrag zijn, wordt bepleit dat studie naar emotieregulatie processen grote aandacht verdiend van onderzoekers op het gebied van gezondheidsvoorlichting en psychologie in de komende jaren. 


\section{Acknowledgments}

The tricky thing about acknowledgements is the nisk of leaving out those who nevertheless deserve your gratitude. Bven though I exercised great care to avoid such omissions, I deem myself human in more ways than this to feel completely sure I have succeeded in doing so. To those who find themselves searching for their names on these final pages I would like to say to trust their feelings if believing they are addressed, yet not mentioned. Know also that I would consider this to be the greatest of my failures. This being said, I would like to express my sincere gratitude to the following people:

First of all to my supervisors, Anton and Nanne. In our discussions I often have found myself on middle ground between you both, which sometimes forced me to proceed on slippery teritory. Nevertheless, I would like to thank you for your patient efforts to leam a stubbom mind think like a social scientist, though I can imagine you to feel - a mutual feeling for that matter - there is still a long way to go down that road. I assure you, however, and in this respect, Anton in particular, that you more than succeeded in evoking an enthusiasm that will last the whole way. Nanne, you've taken pains in eradicating sprouts of extravagant theorizing. These lessons in reductionism will be remembered well, yet obviously, some roots grow deep.

I thank the members of the scientific committee: Gerjo Kok, Agneta Fischer, Bas van den Putte, and Hein de Vries, for the time and energy they spent on reading my dissertation. Also, I would like to thank Tessa en Karien for their assistance with conducting several experiments.

Several people have contributed in a very practical sense to the realization of this dissertation. Taking innumerous little burdens from my shoulders, their help made a world of difference. Whether fixing a crashed computer, making sure I was able to pay the participants in my experiments on time, or generally, clearing larger or smaller troubles from. my path, the support of Leon, José, and Marja was invaluable. 
The "AiO-dip" is a phenomenon familiar to most PhD students. Somewhere halfway, I have lost track of counting mine. Fortunately, on such instances, there always was the company of those that only by gross understaternent I would still merely refer to as colleagues, for several have become ruch more. Bullshitting during lunch brakes, cycling through South-Limburg, taking memorable trips to Barcelona, and getting a little drunk at Themby's or on the beautiful beaches of Maastricht, works like a charm for easing even the most worried of minds. It is particularly at this point in my acknowledgement that I'm too concemed with forgetting names that I hope it will suffice for me to say I'm really going to miss you all and I hope we'll see each other again from time to time.

Of course I thank Bilbo and my father for their willingness to back me up at my defense of this thesis. When (and I explicitly avoid saying if herel) you are in need of a paranymph yourselves, you'll know where to find me.

At some points during the past years, the faith of others has seemed ridiculous to me. On those instances, accomplishment seems so far off that their encouragement may even annoy you from time to time. I know I haven't exactly been at my most charming on these days. It takes family and very good friends, which really boils down to the same thing, to put up with that To my mother and father, Joep, Frank, and Ciet, Tycho, Marc, Charles, Klaas, Sander, Rob \& Marjolein, Stef, and Leon I cannot begin to express my gratitude for them proving me wrong. Cheers to all of you!

You can call yourself lucky to have so many people around you, supporting you each in their own specific way. To have one that does all these things and even more, lucky doesn't suffice anymore... You'd have to call yourself blessed. Emely, diech bis mèt goud neet te betaole. 


\section{Curriculum Vitae}

Rob Nelissen was born on June 25, 1977 in Maastricht In 1995, he received his VWo diploma at the Stedelijke Scholengemeenschap in Maastricht. That same year, he started studying Biology at the University of Utrecht. He graduated cum laude in August 2000, specializing in Behavioral Studies. A change of research disciplines led him back to his hometown, where he started working as a PhD student at the Department of Health Education and Promotion at the University of Maastricht. The research project, entitled: "The role of motivational systems in explaining and influencing health-promoting behavior: $A$ critical evaluation and integration of expectancy-value models", culminated in this dissertation. From September 2005 onward, he will be working as a post-doc researcher at the Department of Social and Economic Psychology at the University of Tilburg. In this function, his research will focus on the role of emotions in decision-making. 
The "Kurt Lewin Institute Dissertation Series" started in 1997. Since 2003 the following dissertations have been published:

2003-1: Femke Bennenbroek: Social comparison and coping with radiation therapy. The significance of different dimensions of comparison

2003-2: Marlene Roefs: Public Participation and Perceived (in) justice in South Africa, $1995-2000$

2003-3: Rick van Baaren: Mimicy: A social perspective

2003-4: Michaéla Schippers: Reflexivity in teams

2003-5: Heleen van Mierlo: Self-managing teamwork and psychological wellbeing

2003-6: Ruben Taris Person-Environment Fit Alongitudinal study of the interaction between employees characteristics and work environmental characteristics

2003-7: Rob Holland: On the structure and consequences of attitude strength

2003-8: Camiel Beukeboom: How mood tums an language

2003-9. Joost Miedema: Faimess and the Self

2004-1: Wolfgang Steinel:Misleading in social decision-making: A motivational approach

2004-2: Edwin van Hooft: lob seeking as planned behavior. In search of group differences

2004-3: Kirsten Ruys: The influence of self and similarity on immediate affective impressions of people

2004-4: Michel Handgraaf: Self-interest and other-oriented motives in social decision making: An ultimatum bargaining perspective

2004-5: Sjoerd Goslinga: Betrokkenheid, participatie en ledenverloop in vakbonden

2004-6: Nymke Frieswijk: Frail, but happy: The importance of self-management ability and social comparison among elderly persons.

2004-7: Jaap Ham: Bridging attribution and spontaneous inferences: Spontaneous and interitional components of dispositional and situational inferences

2004-8: Mirjam Tazelaar: When things go wrong: The influence of noise on interactions

2004-9: Marjolein de Best-Waldhober: Coördinatie in interdependente situaties: Over de afweging van directe uitkomsten versus uitkomsten op lange terming

2004-10: Hinke Groothof: When others are doing better or worse: Responses from the heart and the head

2004-11: Laetitia Mulder: Throwing light on the dark side of sanctions. Sanctioning systems in social dilemmas re-examined

2004-12: Annebel de Hoogh: Personality and charismatic leadership: A matter of context

2004-13: Brigitte ten Brink: Psychological contract: A useful concept? 
2004-14: Gerben van Kleef: Emotion in social conflict. The interpersonal effects of emotions in negotiations

2004-15: Tom Frijns: Keeping secrets: Quantity, quality and consequences

2005-1: Elsbeth Steenland: Stereotype Change: Effects of disconfirming information

2005-2: Annet de Lange: What about causality? Examining longitudinal relations between work characteristic and mental health

2005-3: Reint Jan Renes: Sustained volunteerism: Justification, motivation and management

2005-4: Aloyse Augusta Dinsbach: Socialization in the workplace: A focus on migrant groups

2005-5: Susanne Peters: The social psychology of being better off than others

2005-6: Winny Bakker: Emigration and well-being: The role of personality and cultural identity in acculturation

2005-7: Ruud Zaalberg: The expression of emotion in social situations. The mediating roles of display rules and social motives

2005-8: Catharine Evers: Sex differences in anger expressions. The shaping role of social appraisals

2005-9: Ed Sleebos: The Consequences of Perceived intra-group Respect: The Effects of Differential Intra-group Respect on Behavior and Cognition

2005-10: Jeroen Stouten: Virtue Summons the Funy: Coordination Rules and Faimess in Social Dilemmas

2005-11: Irene de Pater: Doing Things Right or Doing the Right Thing: A New Perspective on the Gender Gap in Career Success

2005-12: Rob Nelissen: Guided by Reason, Struck by Emotion: Integrating Motivational \& Expectang - Value Accounts of Behavior 